\title{
OLDER PATIENT-PHYSICIAN COMMUNICATION: AN EXAMINATION OF THE TENSIONS OF THE PATIENT-CENTRED MODEL WITHIN A BIOTECHNOLOGICAL CONTEXT
}

\author{
by \\ Catherine Anne May Jenkins \\ Master of Arts, Trent University, Peterborough, Ontario, 1996 \\ Bachelor of Arts (Honours), Trent University, Peterborough, Ontario 1984
}

A dissertation presented to Ryerson University and York University in partial fulfillment of the requirements for the degree of Doctorate of Philosophy in the Program of Communication and Culture

Toronto, Ontario, Canada, 2016

(C) Catherine Jenkins 2016 


\section{Author's Declaration for Electronic Submission of a Dissertation:}

I hereby declare that I am the sole author of this dissertation. This is a true copy of the dissertation, including any required final revisions, as accepted by my examiners.

I authorize Ryerson University to lend this dissertation to other institutions or individuals for the purpose of scholarly research.

I further authorize Ryerson University to reproduce this dissertation by photocopying or by other means, in total or in part, at the request of other institutions or individuals for the purpose of scholarly research.

I understand that my dissertation may be made electronically available to the public. 


\section{Abstract \\ OLDER PATIENT-PHYSICIAN COMMUNICATION: AN EXAMINATION OF THE TENSIONS OF THE PATIENT-CENTRED MODEL WITHIN A BIOTECHNOLOGICAL CONTEXT}

PhD 2016

\section{Catherine Anne May Jenkins}

\section{Communication and Culture, Ryerson University and York University}

Drawing on existing theoretical work, as well as field research, this dissertation examines the impact of medical imaging technologies on communication between physicians and older patients when diagnostics often privilege disembodied data over the patient voice. Current diagnostic trends are contextualized within the history of medicine, from Ancient Greece to the present, including the development of imaging. Since the 1970s, advanced medical imaging technologies (e.g., ultrasound, computed tomography, magnetic resonance imaging) have become the diagnostic norm in Western medicine. The rapidity of this shift, which renders the human body as flattened data, can outstrip considerations of the implications of applying such technologies to living patients.

Focusing on older patients, who may be less technologically savvy than younger patients or medical professionals, the field research begins with semi-structured interviews of patients over age sixty-five, exploring their encounters with medical imaging equipment and professionals. This data is interrogated qualitatively using Foucauldian discourse analysis drawing on Andrea Doucet's model of slow scholarship, and informed by Arthur Frank's notion of letting stories breathe; themes were allowed to surface from the patients' narratives, rather than imposed by the researcher. Information emerging from the data considers patients' 
emotions, unexpected physical sensations, communicative strategies and rationalizations, as well as Foucauldian allusions to power. Observational research was also conducted during encounters between physicians and simulated patients in the presence of medical images; these encounters were followed by reflective exit interviews.

Research indicates that although physicians are increasingly trained in patient-centred communication, it is not always optimally practised. Physicians are sometimes more comfortable with the medical discourse of disease than with the emotional, metaphoric language of the patient's illness experience. Since the development of modern Western medicine in Europe of the late 1700s, physicians have been trained to seek pathology, with the increasing aid of medical technologies, rather than listening to their patients. For older patients, who may experience multiple co-morbidities, the lack of communication around advanced medical technologies can increase their sense of vulnerability and anxiety. The dissertation concludes with recommendations for both patients and practitioners to improve communication in the medical context. 


\section{Acknowledgements}

This dissertation was completed with various types of support from many different people, all of whom played some part in making it just a little easier. Those most intimately involved with this dissertation, and who know just how bad I am at time estimates, are my committee. A very special thanks to my supervisor, Steve Bailey, who allowed me to proceed at my own pace and in my own way, who listened to my frustrations and always knew exactly what to say when I was struggling, and who remained positive and supportive throughout the whole process. A warm thanks to Jean Mason, who interviewed me for entry into the Communication and Culture program, saw me through qualifying exams, and has remained by my side every slippery step of the way. Thank you to Jennifer Lapum for providing grounding and the practical medical expertise necessary for this research, as well as making herself available above and beyond the call of duty to oversee the field work, and for the loan of digital recorders.

I would also like to thank the positive influence of previous committee members. Stuart J. Murray, now at Carleton University, helped ease my anxieties about tackling Foucault. He and Bruce Elder both reminded me of my philosophical foundations and helped me sharpen my wits after a lengthy absence from academe.

Others at Ryerson have also lent their invaluable support in many ways. Importantly, Jacqui Schmid, Manager of the Professional Practice and Simulation Lab for Ryerson's Daphne Cockwell School of Nursing, granted me lab access, providing the facility for completing field research. Paul Moore, Director of Communication and Culture, has been a source of insight and advice, as well as a supporter through GA and conference funding. ComCult's admin extraordinaire, Jo Ann Mackie, has kept me on track, sometimes lent an ear, and always had candy available. Other professors supported me through GA or RA work, through which I gained 
valuable insights and experience: Ruth Panofsy, Alan Sears, and Carmen Schifellite. More recently, I have enjoyed the support and wisdom of colleagues at Ryerson's School of Professional Communication, notably Diane Nubla, for her unwavering positive insights, sound strategic thinking, and for being my substitute so I could attend professional conferences.

As a researcher new to digital archives, I appreciated the capable and patient assistance of Ryerson librarians Jay Wolofsky and Don Kinder. I would also like to thank Kelly Kimberley and her staff in interlibrary loans for procuring sometimes obscure titles against all hope. In a similar vein, I would like to thank Emma Sabo of the Learning Resource Centre at The Michener Institute, for helping locate medical imaging sources for phase two field research. The Gerstein Science Information Centre and Thomas Fisher Rare Book Library at the University of Toronto both provided access to difficult to locate texts. Michelle, in Customer Relations at TV Ontario, went above and beyond the call of duty in locating and transcribing a video excerpt that I was unable to access, but wanted to use as an epigraph.

I must also extend thanks to the Rogers family for their consistent support through the Rogers Graduate School Fellowship. The completion of this degree would have been considerably more difficult without their financial support. I would also like to acknowledge support from the Ontario Graduate Scholarship.

Several people at the University of Toronto's Standardized Patient Program (SPP) were instrumental in the success of this research. Most notable are Lorena Dobbie who lent her expertise in role development, and Kathryn Wilkinson for great success in physician recruitment for phase two field research. Diana Tabak and Nancy McNaughton were also helpful in assisting with brainstorming. The simulated patients who assisted with phase two field work, and who shall remain nameless due to confidentiality, have my deepest thanks and respect. Your expertise 
in role-play and simultaneous insightful observation, helped make this research possible. Thanks to my many friends in the SPP who kept the faith and regularly checked on my progress. I would also like to humbly thank the late Dr. Dale McCarthy, whose wisdom and insights provided inspiration.

Although the interview participants must also remain nameless, I acknowledge a debt of gratitude for their willingness to share sometimes uncomfortable experiences, as well as their insights, wisdom, and humour. I would like to thank the physicians who came forward to aid this research, representing diverse practices, and allowing their communicative skills to be put under a microscope.

Through these last several years, I have been fortunate in having people around me, checking on my progress, sharing the triumphs and frustrations. Many ComCult colleagues have provided insight and camaraderie throughout this sometimes difficult journey; most notable among these are Natalya Androsova and Zorianna Zurba. Lifelong friend, poet, and scholar Kathy McConnell provided advice, wisdom, and tangible support when most needed. When I suggested to her that my $\mathrm{PhD}$ was killing me, she readily agreed; true friends do not pull punches. Long-time friend James Spyker ensured that I occasionally got out for a walk, and similarly provided tangible support to help me through. He also gave me the use of his car for extended periods of time enabling me to get out of the city and regain my driving confidence after the accident. Long-time friend Péter Balogh gave me someone with whom to pace as we have undertaken this academic adventure in tandem at different universities, and seen each other through accomplishments and challenges. Long-time friend Philip Eckman listened patiently during many lengthy tea chats, offering insight, wisdom, and the occasional respite on this journey; he also speeded my progress by acting as transcriber. Drawing on her experience as a 
mother and a teacher, my lovely neighbour Dorothy Elkington has led the cheer, and ensured that I stayed on task. I would also like to thank my sister, Elaine Blampied, for worrying about me, and ensuring that I had food in the larder when the going got tough. It also seems fitting to thank my late mother, Isobel Jenkins, for bequeathing me with the sheer tenacity (i.e., bloody mindedness) to complete the $\mathrm{PhD}$. Thanks to you all for keeping the faith, seeing me through, and being on my tag-team as we journey through this life.

A special thanks to Susannah Gray (osteopath), Karen Wingrove (massage therapist), and the Toronto Acupuncture Studio for patiently helping me put my body back together after the accident, and mitigating the effects of too many hours at the computer.

Finally, I would like to thank my second feline triumvirate, for ensuring that I took adequate breaks, occasionally played or ate, and had someone to come home to. For Monte, who stayed strong through the passing of both my parents and accompanied me through the $\mathrm{PhD}$ course work; for Poppi who made it through qualifying exams and the field research; and for little Pear who accompanied me through the writing. 


\section{Dedication}

In loving memory of my Dad,

Tom Cullis Jenkins

(1915-2005)

A brilliant, endlessly curious, passionate, and very special man

and my maternal Grandmother,

Eleanor Martha Irwin née Brown

(1884-1961)

Class of 1915 Nursing Graduate of the

Royal Jubilee Hospital in Kenora, Ontario

Mors rapit omnia

Death snatches all 
Table of Contents

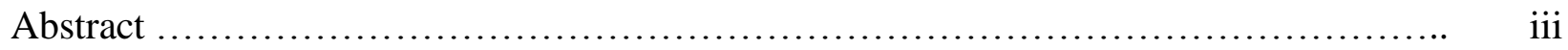

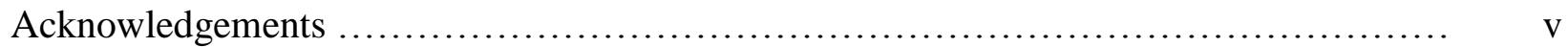

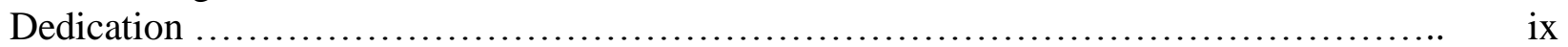

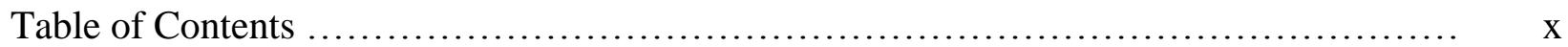

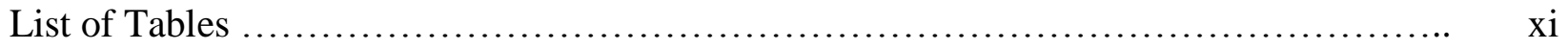

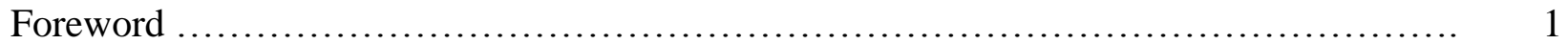

Introduction: Exploring Imperfections in Medical Communication ...................... 9

Overview of Contents ............................................... 15

Chapter 1: How Did We Get Here? Part I: An Abbreviated Archaeology of Medical 17

Practice

The Patient-Centred Approach: The Legacy of Hippocrates ...................................... 19

Biomedicine: The Legacy of Galen .......................................................................... 26

From Medieval to Renaissance Anatomy: Deconstructing the Body ........................ 30

The Modern Era: Professionalized Doctors and Public Health ................................... $\quad 40$

Michel Foucault: The Medical Gaze .................................................................... 46

Conclusion ............................................................................................... 55

Chapter 2: How Did We Get Here? Part II: Advances in Medical and Imaging

Technologies ....................................................................................................... 56

The In(ter)vention of Medical Instruments ........................................................... 58

The Uncanny Magic of Medical Imaging Technologies ......................................... 74

Troubling Images and Imaging .......................................................................... 89

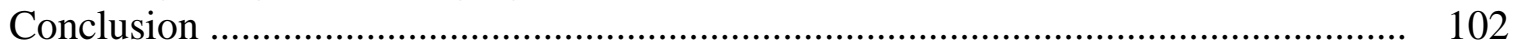

Chapter 3: Methodologies for Exploring Patient-Physician Communication ........................ 103

Research on Patient-Physician Communication …………...................................... 103

A Multivalenced Methodology ............................................................................ 124

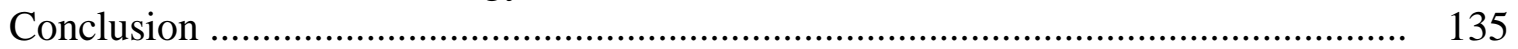

Chapter 4: Older Patients' Imaging Experiences ............................................................. 137

What emotions are described? ............................................................................. 140

What physical sensations are described? ………………................................. 157

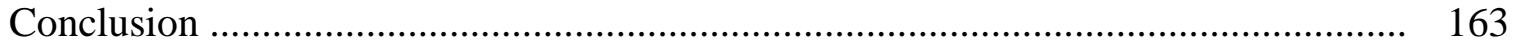

Chapter 5: Older Patients' Communicative Experiences and Rationalizations ................... 164

Formal Information Channels ......................................................................... 164

Informal Information Channels ....................................................................... 179

Media Information ................................................................................ 186

Patients' Reflections and Rationalizations on Imaging ………............................... 189

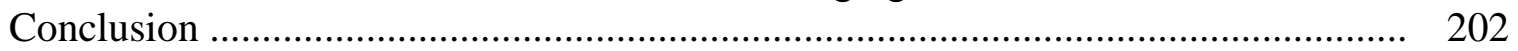

Chapter 6: Older Patients' Responses to Medical Power ..................................................... 204

Physicians' Power ……………………......................................................... 205

Medical Imaging Power ................................................................................... 227

Patients' Power …….................................................................................... 237

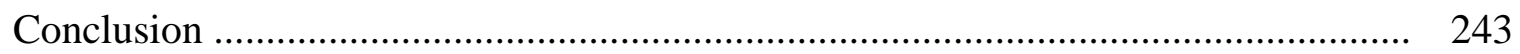

Chapter 7: The Impact of Imaging Technologies on Physician-Simulated Patient

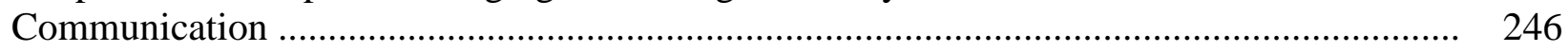

Physician Recruitment ............................................................................... 247

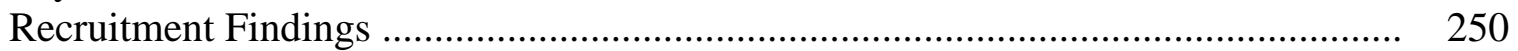


Analysis of Encounters ............................................................................... 254

Lee Berkowitz ………………………………….......................... 255

Dana Jones ....................................................................................... 261

Skyler Hughes .................................................................................. $\quad 270$

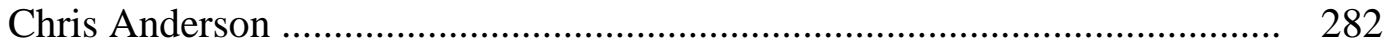

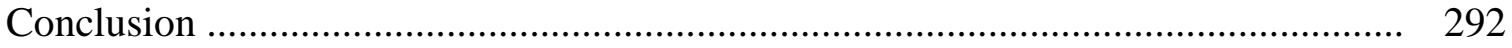

Chapter 8: Simulated Patient and Physician Perceptions of Encounters ............................... 294

Analysis of Exit Interviews ............................................................................ 294

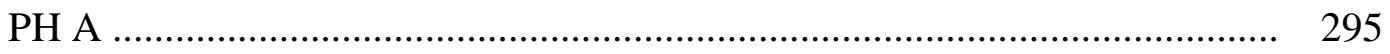

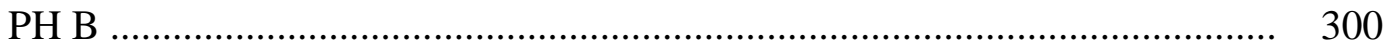

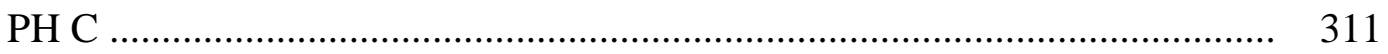

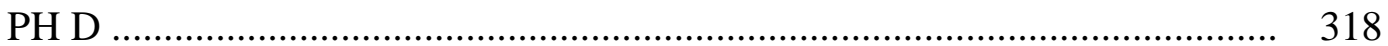

Conclusion ................................................................................................. 331

Conclusion with Recommendations: Where are We Going?............................................. 334

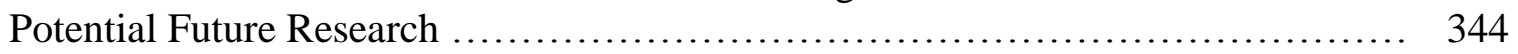

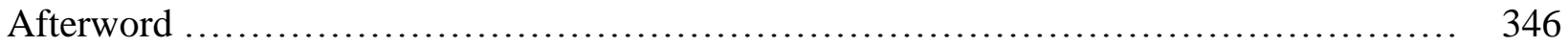

Tables

Table 1: SP-Physician Encounter Schedule ………………………................................. 255

Appendix A: Recruitment E-Mails

\section{Appendices}

Recruitment E-mail for Older Individuals ..................................................... 352

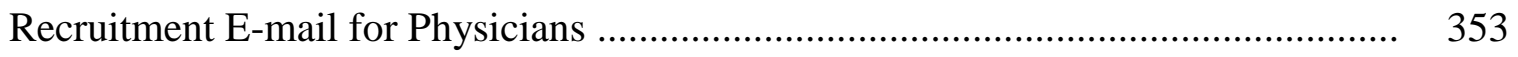

Recruitment E-mail for Simulated Patients .......................................................... 354

Appendix B: Consent Forms

Consent Form for Older Individuals ............................................................. 355

Consent Form for Physicians ........................................................................... 358

Consent Form for Simulated Patients ..................................................................... 361

Appendix C: Semi-Structured Interview Questions for Older Individuals ......................... 364

Appendix D: Simulation Roles, Physician Instructions, and Images

Cancer Role (benign): Lee Berkowitz ............................................................... 365

Cancer Role (findings): Dana Jones ................................................................. 374

Cardiac Role (benign): Skyler Hughes .......................................................... 381

Cardiac Role (findings): Chris Anderson .............................................................. 393

Appendix E: Observation Protocol ................................................................................ 399

Appendix F: Post-Encounter Semi-Structured Interview Questions

Semi-Structured Interview Questions for Physicians ............................................... 401

Semi-Structured Interview Questions for Simulated Patients .................................... 402

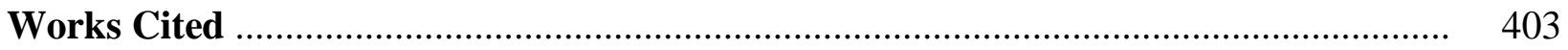




\section{Foreword}

I have this notion that as humans we are most in touch with our animal nature at times of physical change: birth, illness, and dying. Unfortunately, these liminal periods have been medicalized in the last few centuries, so that now when we are our most vulnerable animal selves, we may be confronted by a wall of faceless technology. When we most need human touch, it is sometimes hardest to find; medical technology can obscure our need for human care (Locsin 160). This shift in care also seems to reflect our inability to accept death. We want to retain control, and so we pray to the gods of technology to allow us to control our mortality; in the process we can lose our humanity.

A personal stake should be embedded in a $\mathrm{PhD}$. Why older patients? While I can rationalize, as I do in the Introduction, that this is our fastest growing demographic, the more significant reason is that both of my parents died just before I started this degree. They were both about ninety and, to varying degrees, I had been involved with their care most of my adult life. This significant loss required a cognitive re-ordering of my life. Psychologist Therese Rando connects care giving and anticipatory grief, with post-traumatic stress disorder (38). This complex psycho-emotional site requires a "revision of the assumptive world, the necessity for adopting new ways of being in the external world, and the formation of a new identity incorporating the changes demanded by the deterioration in or loss of the loved one" (Rando 44). The $\mathrm{PhD}$, in both scope and time, offered a way of making sense of this monumental shift in my reality, a way of putting my grief to good and productive use, a way of helping others, and a way to help me find my own feet again.

Why medical communication? I am fully aware of the ideals of patient-practitioner communication, having worked as a standardized patient and patient simulator (SP) for over a 
decade. When I was advocating for my parents, sometimes these ideals were met, but often they were absent. While many potential reasons for this absence exist, the reason I opted to explore further was the increasing presence of medical technologies in the hospital environment.

On several occasions, one or the other of my parents lay in emergency wards, wired to monitors relaying their vital signs to a centralized computer console. While remote monitors enable over-taxed medical workers to observe and record many patients' data simultaneously, increasing fiscal efficiency, monitors may not work optimally, especially on older patients. While heart rate, blood pressure, and respiration rate monitoring seemed fairly reliable, blood oxygen saturation monitors, pinched onto a finger, are not adequately sensitive for patients with compromised circulatory systems, and consistently sound the alarm. Although medical staff were generally quick to respond, initially they would attempt to re-clip the monitor to another site - a toe or an ear. A few minutes later, the alarm would sound again. The investigation of the alarm's cause might be a little slower the second or third time around. After a few unsuccessful attempts, the blood oxygen monitor might be disengaged, or the alarm turned down or off. Meanwhile, parent and daughter would be increasingly concerned about the meaning of the incessant alarm; daughter attempting to explain to exhausted and ill parent at three in the morning in a frenetic emergency ward that everything was fine and the alarm could be ignored.

Although the monitor's screen was angled away from my parent, I could see it and had adequate medical knowledge to make some sense of its squiggly lines and numbers. I found myself observing the output in much the way William Bloom observes his father's in the novel, Big Fish: A Novel of Mythic Proportions: “...after a while it was the machines I was looking at, not my father at all. They had become him. They were telling me his story" (Wallace 171). Especially in the wee hours, on no sleep, in a hyper-vigilant state of exhaustion, machine outputs 
become mesmerizing. Medical technologies mediate the discourse between patients, advocates, and practitioners.

My experience as an SP provided me with both the inside and background knowledge that ethnographer Hubert Knoblauch cites as necessary for undertaking focussed ethnographic research. It also afforded access to other SPs of the appropriate demographic for this research. Senior SPs are trained to critically analyze each encounter and provide articulate and insightful feedback, important abilities for gathering data during post-encounter interviews.

Along this course of study, something more happened. It is hardly uncommon for $\mathrm{PhD}$ students to encounter health problems, although whether this says something about the people who choose this path or something about the acute stress of this path is difficult to determine. In year five, just after I had completed the first phase field research interviews and had started writing the literature review, I experienced a "minor" car accident that left me with whiplash and a concussion, both considered "mild." My mother always told me to be careful what I wished for. Perhaps incessant and intense study of medical discourse caused something in the universe to break loose and drop on my head, in the form of a texting cab driver who T-boned my car. While the accident caused delays in research and writing, it also provided an opportunity for me to experience technologically advanced imaging first-hand. Initial X-rays ensured that there was no damage to my thoracic or cervical vertebrae, although my back and neck were still quite painful several weeks after the accident.

I also experienced acute dizziness, causing concern that I might have a pinched carotid artery. Although I was told that imaging was purely precautionary, I spent a couple of restless nights awaiting the appointment. Pinched how? Between what? An online search only stoked my fears, indicating that a pinched carotid could cause a stroke. A little information is a dangerous 
thing. My doctor assured me that I was not going to have a stroke. When I arrived for the Doppler ultrasound, the technician assured me that if I had carotid insufficiency, I would not be walking around. I thanked him for his reassurance. While the tech was pleasant, once I was settled in a supine position, he explained that I could not talk during the imaging session, as talking would affect the results. So I lay quietly, doing as I was told, because I wanted the session to be over. I was anxious to hear the results, and did not want to do anything to delay hearing them. For most of the twenty minutes, I went into my head for a musical interlude, noting that some of my interview participants had mentioned tuning out in similar ways. When my head was positioned to the left, I was facing the wall, so I closed my eyes. When my head was repositioned, however, I could almost see the monitor. It was somewhat behind me, but straining to see the images, I caught a few glimpses. Before the appointment, I had searched for samples online; as far as I could tell, from my angle, and with no training, everything looked okay. The word "specimen" best typifies how I felt during the Doppler ultrasound, lying there, not moving, not talking, being minutely examined. After twenty minutes of silence, the technician exploring while I lay placidly, he announced that we were done and that I was fine. He added that he hoped not to see me again for a long time. When I visited my doctor the following day, I was told that everything was normal, and we moved on. I was not invited to inspect the images or ask questions about them. This seems the dominant approach of Canadian general or family physicians regarding imaging; patients are not invited to encounter their own images, and images are not part of the discussion about the patient's condition. I discovered later that my family physician only receives imaging reports, rather than the images themselves.

As time passed and my condition showed limited improvement, more imaging was ordered. In an effort to explain the ongoing pain in my lower back, X-rays were taken of my hips 
and pelvis. Although I am well aware of the dangers of X-rays on pregnant women, I noted that prior to X-raying this sensitive female area, no one asked whether I might be pregnant; I knew that I was not, but they did not request that information. Although many would consider me beyond prime reproductive age, I am not post-menopausal. Only after the X-rays were completed did I notice a couple of signs, in English only (in Toronto, a vastly multi-cultural and multilingual city), suggesting that patients inform the technician of possible pregnancy. Again, according to the $\mathrm{X}$-rays, everything was fine, in spite of my lived experience.

Eight months post-accident, my condition declined. I suffered almost constant intense headaches, throughout the day, every day, and over-the-counter pain medication offered no relief. The loud ringing in my ears that had previously ceased, returned. I was also feeling acutely fatigued. When I again complained to my doctor, she suggested several follow-up measures, including an MRI to look for brain damage or some other problem. The initial intake checklist included a specific question about whether I had ever had metal in my eyes. I recalled an excruciatingly painful incident many years ago when a speck of metal dust blew into my left eye from the street. The speck had to be professionally removed at a hospital clinic. Because of this incident, prior to having the MRI, I had eye orbit X-rays taken to ensure no residual metal was present. The orbit X-rays meant two more films, with X-rays shooting through my skull and brain. The reason for this precaution is that an MRI is a giant magnet that would cause any metal within the body to be torn out, causing damage. In total, I received twenty-one X-rays in eight months, probably more than I had received in my entire life previous to the accident.

My MRI appointment was from 11:45 p.m. to 12:45 a.m. on a Saturday night. It was at a hospital in which I had worked as support staff on over one-hundred medical licensing exams, so it was disconcerting being there late at night, and as a patient. As I entered the hospital, the 
realization hit me that it was a cancer hospital. Why was I sent here? Did my doctor suspect something beyond residual concussion damage? Any hospital with the equipment could do an MRI; perhaps this was just the first appointment available.

Even at this late hour, the MRI waiting room felt like an assembly line. The technologist was doing double-duty as receptionist. I was handed a detailed, two-page questionnaire, which included the previous question about metal; I indicated that they should have the orbit X-rays on hand. One of the questions was whether I was claustrophobic. As it had never been tested, I was unsure how to answer. The questionnaire included a comprehensive list of possible things that might be implanted into a body, from replacement joints to piercings and tattoos, anything that might contain even trace metal. Again, the MRI magnet would do soft-tissue damage by dragging these objects through the flesh. I had never seen such a comprehensive list of possible implants, and after the initial shock, I felt very healthy being able to check off no, no, no... Even in the waiting room, I could hear what sounded like a bird's high-pitched cry.

After a brief wait, I was called and led into a changing room. I had intentionally left any jewellery at home. As instructed, I changed into a gown, wearing only socks and panties underneath (bras can contain metal, so are not allowed). I felt de-personalized and vulnerable. Then I was led into an MRI suite where I lay down on the gantry as requested, the massive machine looming over and around my head. A cushion was placed under my knees to provide some comfort. I was handed ear protectors. Once I was settled, a moulded plastic frame was placed around my head, and my head was further wedged in with foam padding to reduce mobility. Then a plastic grill, like something worn by a football player, was snapped down over my face. That was when I felt a wave of claustrophobia. I breathed deeply and it passed. I was handed a rubber bulb to squeeze in case of emergency, i.e., a panic attack. I was told not to 
move, but felt unable to anyway. The technologist left the room, and things went very still. I was aware that I was encased in a mammoth machine, unable to move or readily escape.

I could still move my eyes. Looking down, I saw my abdomen under the gown; noticing my breathing was fast, I consciously tried to slow it down. Beyond my feet, I saw shelves of linens. I realized that the face grill was fitted with a mirrored surface so I could see a reflection of the room behind the machine, and through a shaded glass window, the technicians.

Even with ear protectors, the sound was extremely loud. Although I had interviewed patients about their MRI experiences, it was not what I expected. I felt like I was inside a piece of experimental minimalist techno music, pure slow tones of varying frequencies, as if I were inside a synthesizer. Sometimes the sounds were rhythmic and had a consistent tone; other times two tones would sound, eleven times at a high frequency, followed by eleven times at a low frequency. Sometimes I felt like I was inside a Sensurround speaker at a 1970s disaster movie, with very low frequencies vibrating through my gut. With each sequence, the noise level caused my body to slowly tighten. At some point, I realized that my body was quite tense. I wanted to relax, but afraid that relaxing would be perceived as movement, I maintained this tight, uncomfortable position. I was afraid to swallow. Thought I might cough. I controlled normal impulses. Felt like a bug under a microscope, a specimen, an object. I experienced a sensation of something moving across my head from one ear to the other, like a change in pressure. I could feel each slice the MRI made through my body, evoking unpleasant images of the Visible Human Project.

I was ready for the session to be over. After forty minutes of restricted movement, I began to feel bored. Afterwards, I felt strangely disoriented and dizzy. The machine's vibrations 
set off a sympathetic tone in my right ear. It being late at night, and me feeling tired, did nothing to help my sense of vulnerability. I was home before one in the morning.

Reflecting on the experience, I wondered about my unquestioning compliance. Rendering one's body so still for so long is harder than it sounds. Having my head immobilized was unpleasant. Although it was not as bad an experience as I had feared it might be, I would prefer not to have another MRI.

A week later, I woke from a nightmare that I was taking part in my own dissection. I was conscious of trying to keep my stomach muscles relaxed as I raised my shoulders from a supine position to operate on my open guts. Imaging is not a benign activity. 


\section{Introduction:}

\section{Exploring Imperfections in Medical Communication}

We look for medicine to be an orderly field of knowledge and procedure. But it is not. It is an imperfect science, an enterprise of constantly changing knowledge, uncertain information, fallible individuals, and at the same time lives on the line.

Atul Gawande (7)

Communication is, by nature, imperfect. Yet we rely on this complex and messy process daily in many aspects of our lives to exchange information. We tend to use it uncritically, to take it for granted. Yet in some professional practices, the ability to communicate effectively, and as accurately as possible, is essential, even if it is sometimes overlooked; medical practice is a prime example. Medicine is a unique scientific site, in that it involves an intimate human interaction requiring a great deal of trust, sometimes marked by a strained power dynamic. Medical education teaches students complex technical tasks and how to synthesize data, as well as how to communicate with patients. Once in the field, residents must learn to simultaneously master both technical and communicative skills. Astute students do eventually manage this demanding multitasking, but it can take years of practice and continual learning to master seamlessly. In the complex medical interaction, physicians and patients must find a common language that enables them to comprehend each other and generate mutually understood and agreed upon treatment options. Underlying medical practice, however, are centuries of pedagogy in which both physician and patient have been trained to accept their given roles. The medical interaction is complicated by a number of factors: a biomedical practice model that can objectify patients; the evolution of a unique medical language; the rapid growth of technologies in the clinical context; and increasing pressure on medical practitioners to treat patients with optimal efficiency in consideration of fiscal restraint (at least in the current Canadian system). 
The tension between patient-centred and biomedical practices seems to have existed from the inception of Western medicine, as exemplified by the differing perspectives of Hippocrates and Galen towards their patients. The focus in this dissertation is on the development and practice of Western medicine, from Greece, through Europe and into North America, and sometimes specifically Canada. Although different medical practices evolved in Asia and other regions, these practices are beyond the scope of this research. By the 1700s, with the professionalization of medicine in Europe, it becomes increasingly clear that some doctors prefer to puzzle over objectified medical data representing cases, rather than to consider their patients as people. While certain physicians have always spoken of the wisdom of listening to patients' voices and illness experiences, a louder voice has often countered with new medical technologies with which to gather more "objective" evidence from patients' bodies. This objectification of patients by physicians may leave patients feeling that there exists a communicative rift, and that they are the objects of their physicians" "clinical gaze" (Foucault Birth of the Clinic 103). Physicians may also feel caught between the complex biomedical demands of their work and an expectation to forge strong therapeutic alliances with their patients through effective communication.

This dissertation explores communication between patients and physicians during the complex medical interchange in the presence of advanced imaging technologies (both the scanners and the resultant images). Because biomedical technologies have encroached into the clinical context gradually, adding to a multiplicity of competing demands, medical practitioners are not always fully conscious of the impact that this growing technological presence has on their attempts at patient-centred communication. While the physician may be having a stressful day at 
work, the patient may be having a life-altering or life-threatening experience, potentially without warning; the stakes are radically different for the individuals on either side of this conversation.

To further define the site of study, the patient population under consideration is older adults. According to Statistics Canada projections, 21\% of Canadians will be over age sixty-five by 2026; this is the fastest growing demographic in Canada and globally (World Health Organization). Due to age, this demographic is more likely to require medical attention, often exhibiting multiple co-morbidities. Yet older individuals may be less technologically acclimatized than those who have been raised and trained in high-tech societies - a population that includes medical residents and many practicing physicians_-and, consequently, older patients may feel uncomfortable in a high-tech medical context that subjects them to a radically different practice model from which they grew up. Although profound biomedical advances enable the prolongation of life, patients' broader care needs may not be addressed. Older adults may feel stripped of their sense of agency and intimidated, frightened, or unable to question the high-tech contemporary hospital environment during illness. As younger generations acclimatized to advanced technologies begin to age and have increased medical care needs, this sense of feeling overwhelmed by the technology may be less profound; however, for current elders, those already in this demographic, it is imperative that physicians and patients recognize the potential communicative problems present in an increasingly technologically dominated medical environment.

The primary theoretical perspective of this dissertation is that of Michel Foucault's The Birth of the Clinic: An Archaeology of Medical Perception (1973). This work offers a critical look at the foundational perceptions and historical development of the patient-practitioner relationship, acknowledging a dominant biomedical bias. Foucault's analysis indicates that 
hospitals of the late 1700s offered physicians opportunities to observe multiple patients through a disease course, into death, and then into post-mortem investigation. For Foucault, "the great break in the history of Western medicine dates precisely from the moment clinical experience became the anatomo-clinical gaze," in the late 1790s (BC 179). In its most reductive form, the clinical gaze causes patients to feel objectified; "in relation to that which he is suffering from, the patient is only an external fact" (BC 107). Foucault, however, also supports a more complex understanding of the clinical gaze. "The object of discourse may equally well be a subject, without the figures of objectivity being in any way altered" ( $B C \mathrm{xv})$. When the clinical gaze objectifies, power shifts towards the physician; if, however, the facts of the disease state are coproduced by physician and patient, power can be somewhat more equally shared.

Although The Birth of the Clinic is recognized as a philosophical or sociological work, its theory can readily be applied to medical ethnographic research. Research theorist John Cresswell states, "ethnography is a way of studying a culture-sharing group" (68). Medical culture is cocreated by patients and physicians, hence the use of Foucault's work as the theoretical basis for an ethnographic study of a shared medical culture seems a natural fit. The presence of advanced biomedical technologies in current practise has an impact on patients and practitioners, as well as their communication. Bringing a Foucauldian lens into this setting will aid the examination of the biotechnologically enhanced clinical gaze from the perspectives of both patients and practitioners, as well as help to ascertain the shifting power relations over the contemporary medicalized body.

The primary questions guiding this research are: 
How does the tension between the patient-centred approach and biomedical technologies affect patient-physician communication, especially when older patients are concerned?

$>$ Is it possible to optimize practitioner use of biomedical technologies while retaining a patient-centred approach to healthcare?

These questions are explored using a combination of secondary and primary research. Secondary research includes an examination of medical history, drawing primarily on Roy Porter, and medical and imaging technologies, based on Stanley Joel Reiser's writing. Works discussing various aspects of patient-practitioner communication are also incorporated, as well as methodological texts.

The first phase of primary research consists of a series of interviews with older patients regarding their medical imaging experiences. This data is interrogated qualitatively using Andrea Doucet's model of "slow scholarship," and informed by Arthur Frank's notion of "letting stories breathe"; themes surface from the patients' narratives, rather than the researcher imposing an order. Information emerging from the data considers patients' emotions, physical sensations, communicative strategies and rationalizations, as well as Foucauldian allusions to notions of power, although there is overlap between these categories.

The second phase of primary research is an observational study of encounters between simulated patients (SPs) and physicians, in the presence of digital and hard copy medical images. SP roles are developed from the narratives gathered during phase one field research to optimize believability. SPs, sometimes used in medical educational and research, are employed to avoid repeatedly subjecting patients to the devastation of hearing bad news - something for which it would be difficult to gain ethics approval. As with patients, these SPs are free to respond to a 
situation in the moment; for instance, if a physician uses technical terminology, the SP might ask for clarification to ensure understanding. The use of SPs also underscores that the focus of these encounters is the communicative dynamic between two individuals, a professional and a lay person. An observation protocol developed for this research is used to interpret the encounters. Exit interviews with the physicians and SPs are also conducted. By providing a deep analysis of patient-physician communication, with the intention of improving clinical communicative practices, this research offers important practical solutions to our stressed medical system. This research potentially impacts both medical practice and medical education. The hope is that some of its findings and recommendations might be extrapolated for use in communicating around various medical technologies; however, for the sake of this dissertation, imaging provides ample material and a defined focus. This research should draw physicians' attention to the impact of biomedical technologies on patient-physician communication, and enhance communication strategies for patient interactions in the presence of biomedical technologies. Additionally, this research may serve as a basis for developing roles for medical educational encounters with SPs to increase student comfort with communication in the presence of advanced biomedical technologies. The aim of this investigation is twofold:

to theorize and analyze complex verbal and non-verbal patient-physician communication in an increasingly technological healthcare environment, with a focus on older patients, and

to act as a catalyst for enhancing communication strategies that are patientcentred, but functional within the contemporary biomedical technological framework. 
The Toronto consensus statement on improving patient-physician communication, drafted by a group of physicians in 1991 states that: "Although a great deal is known about the early phases of the interview, less is known about information exchange and therapeutic strategies" (Simpson et al. 1386). Sociologist Mildred Blaxter notes that, "there is still a lack of ethnographic evidence from the patient's perspective on critical stages of the diagnosis and treatment of serious illness, which might show whether or not patients do in fact feel themselves 'disappearing' behind the image" (Case of the Vanishing Patient? 764). Her study concluded that imaging technologies provide information and comfort to patients; however, the lack of clear, consistent, and coherent practitioner communication around diagnostic technologies can be alienating. Patients sometimes feel excluded from diagnostic information or treatment options. It is precisely at these junctures, when patient and physician communication is mediated by advanced medical technologies, that this dissertation research focuses.

\section{Overview}

Chapters 1 and 2 offer a review of literature and medical history, to position the field research. Chapter 1 examines medicine from its Western roots in Ancient Greece, to the beginnings of modern medicine. This chapter also explores Foucault's theories of the clinical gaze and power dynamics useful to this study. From this brief exploration, it becomes apparent that the split between patient-centred and biomedical medicine exists from the outset of medical development.

Chapter 2 continues to examine modern medical history with the development of medical object and imaging technologies. Additionally, this chapter critically examines the efficacy of imaging in contemporary diagnostics. 
Chapter 3 offers the field work methodology, beginning with a longer critical discussion of patient-centred practice, and completing the literature review. Andrea Doucet's "slow scholarship" approach is wedded to Arthur Frank's notion of "letting stories breathe," allowing themes to emerge from the data, rather than imposing the researcher's ideas. The field work approach is then detailed.

Chapters 4, 5, and 6 provide analysis of phase one interviews with older patients regarding their imaging and medical communication experiences. Chapter 4 provides an exploration of the complexities of patients' emotional responses to the imaging experience, as well as physical sensations sometimes encountered. Chapter 5 provides an examination of older patients' communicative experiences during imaging, considering formal, informal, and media channels, as well as patients' reflections and rationalizations regarding imaging. Chapter 6 offers a deeper discussion of the Foucauldian notion of power, including shifting dominant powers in the medical context: physicians, medical imaging, and patients themselves.

Chapters 7 and 8 provide analysis of the encounters of physicians with simulated patients, in the presence of images. Chapter 7 begins with a brief discussion of findings during physician recruitment for this research, and then provides analysis of physician-simulated patient encounters using the observation guide created for this study. Chapter 8 provides an examination of the exit interviews with both physicians and simulated patients.

The Conclusion offers an overview of findings, as well as recommendations for both patients and physicians. Appendices containing field work documentation and research tools complete this dissertation. 


\title{
Chapter 1: How Did We Get Here?
}

\section{Part I: An Abbreviated Archaeology of Medical Practice}

\author{
Traditional history of medicine simply ignored the patient. ${ }^{1}$ After all, it was what \\ the doctor did to, and for, the sick that counted; the patient was just the raw \\ material, the unwitting bearer of a disease or lesion.
}

\section{Roy Porter, Patients and Practitioners 2}

This dissertation foccusses on two dominant models of contemporary Western medical theory and practise: the patient-centred model and the biomedical model. As its name implies, the patient (or person)-centred model acknowledges the patient's experience of illness. Using dialogue with open-ended questions, physicians allow patients to tell their own stories, thereby gaining an understanding of the patients' perspectives of their diseases. This approach allows physicians to learn their patients' symptoms, while simultaneously examining potential causes and the psycho-emotional impact of an illness. In the biomedical, or scientific, model, the disease state is the physician's primary focus and, as medical historian Roy Porter implies above, the patient is merely a Petri dish, a vessel containing the true object of interest. Advanced medical imaging technologies developed in the latter half of the twentieth century, and now in common use, reinforce the biomedical model. Examining the relationship between two communicative poles, the primary questions guiding this dissertation are: How does the tension between the patient-centred approach and advanced biomedical imaging technologies affect patient-physician

\footnotetext{
${ }^{1}$ The word "patient," as we now understand it in the medical context, comes into existence in the late 1300s and is not commonly used until the 1800s (Porter PP 296). In Porter's study of diaries and letters from the 1700s, the discussion is about people who are ill, rather than patients; Nicholas Jewson discusses the shift away from the "sickman" that takes place between 1770 and 1870, coinciding with the move away from bedside medicine and towards hospital medicine (235). This shift in language is noteworthy as it underscores the change in power dynamics with the professionalization of medicine. For ease and clarity, the word patient will be used throughout this dissertation, even when discussing historical contexts predating the term's popular use.

A prime example of the type of "traditional history of medicine" that Porter alludes to is Erwin Ackerknecht's A Short History of Medicine (1968). This text offers a chronology of medical discoveries and the men behind them, with little acknowledgement of the role of patients.
} 
communication, especially where older patients are concerned? Is it possible to retain a patientcentred approach to healthcare while optimizing practitioner use of biomedical technologies?

A balance between the patient-centred and biomedical approaches helps create an alignment of agendas and a theraputic alliance, optimal for patient care; however, an examination of highlights from the history of medicine reveals that patients have often struggled to be heard. This tension between the patient-centred and biomedical models has existed since the beginning of medical practise, with the balance occasionally shifting depending on conceptual and methodological changes through different cultures and eras. Historically, how was the patient perceived by the Western medical professional? How does the historical perception of the patient continue to inform medical theory, education, and practice?

As is reflected in this chapter's title, this is an attempt at something more than a simple history of medicine, but rather, what Michel Foucault called an "archaeology." In addition to the notation of specific past events, also present is an analysis of the ways in which past medical habits impact present practice. The perception of the present in attempted isolation gives a naive half-picture; the present can only be fully apprehended through an understanding of what came before. In Discipline and Punish (1975), Foucault states that he was, "writing the history of the present" (31). He also suggests that a study of "Differences in historical developments and institutions would make a detailed comparative examination too burdensome" for his study, but this opens the door to the possibility that others might explore the archaeology of other institutions or different aspects of the institutions that Foucault studied (DP 309). In some small way, this dissertation attempts to add to Foucault's work in the archaeology of medicine, by examining a specific aspect of the complex communicative dynamic between physicians and patients when medical imaging is also present. 


\section{The Patient-Centred Approach: The Legacy of Hippocrates}

The greatest mistake in the treatment of diseases is that there are physicians for the body and physicians for the soul, although the two cannot be separated.

Plato (c. $428-$ c. 348)

Hippocrates $(c .460-c .377$ BCE) espoused a patient-centred approach, based on careful observation (Porter, Greatest Benefit to Mankind 56; Wootton 29). Prior to Hippocrates, the Greeks, like other Ancients, supposed that the causes of disease were metaphysical; ailments were treated with magic, an array of herbal and animal concoctions, and the intervention of sacred healers calling on gods like the caduceus-carrying Asclepius, and his daughters, Hygeia and Panacea (Porter, GBM 51-53). From the sixty or so remaining volumes of the Hippocratic corpus, ${ }^{2}$ it is apparent that Hippocratic physicians took a more rational, organic, systematic approach to disease, its origins, and the treatment of patients, than had their predecessors. Separating disease from religion was revolutionary, and helped establish a social position for physicians, albeit it in an unregulated profession. In a field of competing healers including sorcerers, soothsayers, charlatans, exorcists, shaman, priests, and surgeons, the Hippocratics proclaimed a uniquely earth-bound, intellectual medical system (Porter, GBM 53; Wootton 29).

Based on concepts of natural medicine alluded to by Empedocles, and already established in Ancient Greece, China, and India, Hippocrates developed humoural medicine, and his ideas were later transcribed by Polybus in The Nature of Man (Porter, GBM 58, 54; Wootton 36). Based on observable surface anatomy, Hippocratic humoural medicine was arguably hampered by its lack of understanding of deeper human anatomy and functioning. The Greeks declared the

\footnotetext{
${ }^{2}$ The academic consensus is that none of these books was actually written by Hippocrates, but by a series of scholars over about two hundred years. They were gathered together for the first time $c .250 \mathrm{BCE}$ in the Library at Alexandria (Porter GBM 55-56; Wootton 29).
} 
human body sacrosanct, even after death, and dissection was perceived as such a gross indignity that it was never performed, even on prisoners of war. Anatomical knowledge was derived only from the wounded — war and the gymnasium provided ample practice in tending wounds and setting bones - or by analogies to butchered or dissected animals (Porter, GBM 56; Wootton 30, 42). As a result, ideas of how the human body functioned and healed were largely speculative. Writings of one Hippocratic physician, which seem to express common belief, indicate that: "Every part of the body which is covered by flesh or muscle contains a cavity. Every separate organ, whether covered by skin or muscle, is hollow, and in health is filled with life-giving spirit; in sickness it is pervaded by unhealthy humours" (cited in Wootton 30). Disease was caused by an imbalance of the body's humours, and the role of the physician was to gently guide the patient back towards a state of equilibrium. The unbalanced body sometimes ridded itself of excess humours. Sick people vomited to diminish surplus yellow bile, coughed up mucous to reduce phlegm, and had nosebleeds to purge an overabundance of blood (Porter, GBM 56-57; Wootton 31-33). Black bile, now recognized as a symptom of internal bleeding, was a later addition, creating what are commonly known as the four humours. These four humours corresponded to the four seasons, primary qualities, ages of man, and temperaments; each humour was more prone to imbalance during certain seasons, weather conditions, times of life, or moods (Porter GBM 57; Wootton 36). While some humoural excesses might be rebalanced naturally, others required medical intervention.

Medical diagnosis was accomplished by careful observation of, and discussion with, the patient to ascertain his symptoms and lifestyle - a patient-centred approach. Physicians' examinations went well beyond ascertaining the patient's age, lifestyle, and palour, to include mood, thoughts, dreams, speech patterns, mannerisms, and even hair colour, to aid in the 
diagnosis (Porter, GBM 60; Wootton 34). As the concept of contagion had not yet been established, an individual patient's humoural flux was the primary diagnostic indicator. With a holistic understanding of the human body, but limited knowledge of its integrated parts, examination of patient eliminations was essential (Porter, GBM 56; Wootton 8, 33). Once the patient was diagnosed, the physician would proclaim a prognosis, based on his experiences of similar humoural imbalances in other patients. To avoid blame for causing death, announcing a prognosis was especially important when the patient's demise was imminent; physicians were particularly adept at identifying symptoms of looming death (Porter, GBM 61-62; Wootton 35).

Treatment options were conservative and limited in Hippocratic medicine; the role of the doctor was primarily to observe and commiserate, to watch and wait. The most common prescriptions were for alterations in diet or exercise, based on what became known as the Law of Hippocrates, that "opposites are cured by opposites" (Wootton 31); for instance, an obese patient was told to eat less (Porter, GBM 59). While changes in diet, exercise, sleep, and hygeine might prove helpful in alleviating the effects of some ailments, they would have had little impact on prevalent diseases like the plague, smallpox, leprosy, typhoid, or other common infections (Porter $G B M 10$ ). Medical practice was generally passive, acknowledging the body's natural ability to heal itself, or fail in the attempt, with the physician's guidance. Herbal remedies were less common in Hippocratic medicine than in other Ancient practices, but might be prescribed to induce a reduction of vile humours; emetics promoted vomiting, while purgatives induced diarrhea. Cautery ${ }^{3}$ and bloodletting were more complicated interventions, more common in Galenic than Hippocratic practice, especially as the Hippocratic prohibition against cutting

\footnotetext{
${ }^{3}$ The practice of cauterization may have been an Arabic influence on Western medicine. After Hippocratic and Church prohibitions against surgery, cauterization provided an alternative, notably for the removal of tumours (Johnson 173). Although the first of the Ancient remedies to be discontinued, cauterization was still used into the 1800s. René Laennec (1781-1826), inventor of the stethoscope, reportedly prescribed the application of heated copper rods to the chest to treat tuberculosis (Wootton 31).
} 
prevented its physicians from performing venesection. While the use of emetics, purgatives, and bloodletting predate Hippocrates, he was responsible for rationalizing their use and promoting their medical efficacy, although, as historian David Wootton argues, these treatments did "more harm than good" for centuries of patients $(2,31)$.

Editions of Hippocratic works continued to be published for use in medical education into the modern era, with a new English translation in 1849 (Wootton 6). The Hippocratic Oath, outlining medical ethics, was reintroduced into medical schools during the sixteenth century, cementing Hippocrates's continued influence. Since the late 1940s, some version of the Oath has been universally employed by medical schools upon graduation, although it has been modified to reflect contemporary attitudes (Wootton 5,6). While concepts like helping patients to the best of one's abilities, avoiding sexual contact with patients, and confidentiality remain, schools have removed the prohibition against surgery; some also omit the prohibition against abortion, although Porter suggests that the practice was common in Ancient Greece, in spite of the Oath (GBM 62-63). As Wootton asserts, our romantic notion that the Hippocratic Oath represents some long, continuous lineage of the medical profession, was created in the modern era after it was reinstated (6). The Hippocratic idea of a patient-centred approach has had a similar resurgence.

The modern movement towards a more patient-centred approach coincided with the introduction of new technologies, when available medicines were still largely ineffective. The primary role of the physician was to call on patients and listen to their woes; however, this role had been undermined by the urbanization of the industrial revolution, the creation of medical specializations, and centralized hospitals. According to Porter, the "patient-as-a-person" movement began in the 1880 s with addresses by various medical practitioners emphasizing that 
the role of the physician was to treat people, rather than diseases (Cambridge History of

Medicine 123). ${ }^{4}$ The introduction of non-medical technologies, like automobiles and telephones, reduced stress on physicians; cars allowed doctors to cover a broader territory more efficiently, while telephones allowed them to assess the urgency of medical situations and begin treatment without being physically present (Porter, CHM 126, 129). In the United States, house calls dropped from 50\% in the 1920s to $2 \%$ by 1990 (Porter, CHM 130). Oddly, this drop coincided with a change in the public perception of illness, causing people to seek medical attention with increasing frequency, in spite of overall improvements in public health. ${ }^{5}$

Antibiotics, such as sulpha drugs (1935) and penicillin (1941), mark the first biomedical intervention to effectively treat patients' diseases $^{6}$ (Porter, CHM 133; Wootton 21). After centuries of philosophizing, theorizing, or actively harming patients, doctors' abilities to finally do something medically useful for their patients, has, according to Porter, led to an unfortunate change in physicians' attitudes:

...although doctors have become therapeutically far more awesome than ever before, they have ceased giving the patients what they want. Effective against disease at an organic level, doctors have often found it no longer necessary to enlist the psychological benefits of the doctor-patient relationship in bolstering the

\footnotetext{
${ }^{4}$ In an 1882 address in Vienna, Dr. Hermann Nothnagel stated: "I repeat once again, medicine is about treating sick people and not diseases." At influential Johns Hopkins University, Canadian Dr. William Osler (1849-1919) stated: "The good physician treats the disease but the great physician treats the patient who has the disease." Dr. Canby Robinson, one of Osler's students, wrote The Patient as a Person (1939) urging the holistic treatment of patients and cautioning that “'scientific satisfaction' was replacing 'human satisfaction' in medicine" (cited in Porter, CHM 123, $124,125)$.

${ }^{5}$ In the US between 1928 and 1931, people visited their doctors an average of 2.9 times per year; by 1964, that number had increased to 4.6 times per year, and in 1990, it was 5.5 times per year. Similarly in the UK, in 1975 the average number of doctor visits was three per year, and by 1990 it had increased to five per year (Porter, CHM 132). Given these increases in the US and UK, it seems safe to assume that Canadian statistics are similar. Owing to the differences in the healthcare systems between the US and UK, this increase cannot be blamed on greedy insurance companies.

${ }^{6}$ Vaccines for various diseases were developed beginning in the late 1800s; however, these represent prophylactic medicines rather than disease treatments.
} 
patient through an illness. The whole patient-as-a-person movement fell into desuetude after 1950, replaced by a new generation of physicians filled with an overweening therapeutic self-confidence. The aspects of the doctor-patient relationship to which patients had once thrilled, such as the physician's show of interest in the history-taking or the laying on of hands in the physical exam, became downplayed in favour of using the resources of diagnostic imaging and of laboratory tests in the diagnosis of disease. It was not that physicians became somehow more inhumane, merely that the previous display of apparent humanity had now become therapeutically unnecessary (CHM 134).

Patients' stories of illness are still important to physicians for diagnostics, however. A study by J.R. Hampton et al. indicates that in sixty-six out of eighty patients (82.5\%), the discussion with the patient provided adequate information to reach a correct diagnosis; in the majority of cases, the physical examination and lab tests were only helpful for confirmation (489). This unfortunate turn that Porter alludes to is underscored by the marked reduction in patient satisfaction during clinical enconters, and complaints by patients of feeling like "a piece of meat to be prodded, punctured, and otherwise ignored," caught up in a dehumanized healthcare system (Leder 24).

Contrary to Porter's assertion that the patient-as-a-person movement died in the 1950s, there seems to have been a continuation of this approach, reinvigorated by psychologist Carl Rogers's Client-Centred Therapy in 1951. Rogers's ideas, developed for psychotherapeutic work, seemingly filtered into other medical practices, supporting a patient-centred approach that has been proclaiming patients' rights since the 1960s (Laine and Davidoff 152). Patient-centred practice evokes a three-dimensional, thinking, acting, feeling subject who interacts with the physician. A patient-centred practice acknowledges the patient as a subject having an illness 
experience, exploring the impact of the disease on the individual, as well as that patient's understanding of the disease and treatment options. Ideally, it also explores the potential impact on the patient's work, social, or familial contexts, as well as what supports are available to the patient. In their review of existing literature, Nicola Mead and Peter Bower identify five concepts common to all definitions of the patient-centred approach: "biopsychosocial perspective; 'patient-as-person'; sharing power and responsibility; therapeutic alliance; and 'doctor-asperson"' (1087). The move towards patient-centred practice is not, however, without controversy; while Paul Little et al. declare that this style of practice "can improve satisfaction and biomedical outcomes," other research shows an increase in patient satisfaction only, not in biomedical outcomes (Little et al. 468; Lee and Lin 1816; Kinnersley, Stott, Peters, and Harvey 716). Lee and Lin's research also acknowledges the need for physician flexibility in communication styles to accommodate the high variability of patient needs for autonomy, information, and advice (1812).

While the patient-centred approach seems to increase patient satisfaction, it also requires a complex communicative practice from physicians, one that may be difficult to undertake when simultaneously performing necessary biomedical work in a clinical context. Medical schools do not generally train students to multi-task in these two complex areas simultaneously. As Annemarie Mol and John Law declare: “...the living body is both an object and a subject” (87). While physicians observe, measure, and palpate, they must not lose sight of the fact that they are enacting their practice on a living, experiencing, and private subject-body. 


\section{Biomedicine: The Legacy of Galen}

We doctors have always been a simple, trusting folk! Did we not believe Galen implicitly for fifteen hundred years and Hippocrates for more than two thousand years?

Sir William Osler (1849-1919)

The term "biomedicine" is a combination of the words biology and medicine, coined in the twentieth century, and certainly in use by the $1960 \mathrm{~s}^{7}$ Biomedicine is defined in The Canadian Oxford Dictionary (1998) as: "the application of biology to clinical medicine," wherein biology is: "the study of living organisms"; clinical means, "of or for the treatment of patients"; and medicine is "the science or practice of the diagnosis, treatment, and prevention of disease." Hence, biomedicine might be defined as the application of the study of living organisms to diagnose, treat, or prevent disease in patients. This is quite different from David B. Morris's more broadly constructed "biocultural" model of disease, in which postmodern illnesses are "situated at the crossroads of biology and culture" (71).

Dictionaries use the passive voice, the same grammatical construction used in most scientific writing, which avoids identifying the actor applying this knowledge or treatment to the patient (Koutalos 207-211). While the actor might be defined as a health-care practitioner, this term is too broad, as it includes those in support roles and alternative practices who may not practice biomedicine. The word physician speaks to a more closely defined group, who most frequently practice biomedicine in the contemporary medical context, although the term has been used across cultures for centuries. While The Canadian Oxford Dictionary defines physician as: "a person legally qualified to practise medicine," this is a modern characterization post-dating

\footnotetext{
${ }^{7}$ The exact year of origin seems illusive, as different dictionaries cite 1926, 1945-50, 1961, and 1963.
} 
the founding of various professional organizations and the Flexner report ${ }^{8}$. The words physician or doctor, titles for healers since the ancient Greeks, did not initially connote a legal or licensing aspect (Porter GBM 77-78).

The Western biomedical tendency is rooted in the work of Galen $\left(129-c .216^{9}\right)$ (Porter GBM 8). Galen codified medical knowledge on topics including botany, pharmacology, diagnostics, philosophy, pedagogy, and anatomy ${ }^{10}$ in his voluminous corpus. ${ }^{11}$ Although many volumes were lost over time, those that survived came to form the core curriculum of Western medical education, influencing generations of teaching and practice until at least the 1500s (Porter GBM 73; Wooton 5). Some volumes, for instance Galen's work on venesection (bloodletting or phlebotomy), saw new translations published in the mid-1800s; clearly, Galen was still influential in teaching and practice into the modern era. ${ }^{12}$ In spite of the growth of medical knowledge beginning in Renaissance Italy in the late 1400s, and overwhelming evidence of the potential harm of some ancient techniques, many physicians continued to rely on Galen, often to the detriment of their patients. Although knowledge about the human body expanded,

\footnotetext{
${ }^{8}$ Flexner's Medical Education in the United States and Canada (1910), completely reshaped, standardized, and codified medical education in North American and beyond. The report also had the effect of closing dozens of substandard institutions, including many that had been open to women and black students (Berliner; Porter, GBM 358). ${ }^{9}$ Ackerknecht suggests 130-201 as Galen's dates (Ackerknecht 75).

${ }^{10}$ Born in Greece, but Roman by choice, Galen fell under the bans on human dissection instituted by both states, where the human body was considered Divinely created, and therefore, sacrosanct. To feed his anatomical curiosity, Galen substituted primates and other non-human animals for human cadavers, introducing numerous errors that remained unchallenged until Vesalius (Aufderheide 5; Porter GBM 75; Wootton 45).

${ }^{11}$ According to Ackerknecht, the Galenic corpus was comprised of twenty-two volumes and over one-hundred treatises, while Porter alludes to the sixteen Galenic texts used in Alexandrian medical teaching by the year 500 and the translation of 129 works into Arabic (Ackerknecht 75; Porter GBM 90, 95). Nearly two-thousand years later, it is impossible to know precisely the full depth of the Galenic corpus, but it is apparent that Galen was extremely prolific, and at a time when text was necessarily hand-scribed. His prodigeous output helps account for his long-term popularity.

12 Ackerknecht reports that France imported 42-million leeches for bloodletting in 1833 (151). Clearly the practice was still common and popular, in spite of advances in medical knowledge and empirical evidence that bloodletting could be fatal. Although there was much debate regarding venesection over the centuries, discussions tended to focus on the correct site and duration of bleeding, rather than questioning its efficacy and dangers (Kuriyama 19; Porter GBM 171; Vesalius 1969 (orig. 1539)).

Phlebotomy is still used for treating certain conditions which cause an overproduction of red blood cells, for instance polycythemia vera. Leeches are currently used in microsurgery to encourage blood flow into damaged tissues.
} 
and the ability to ascertain prognosis improved, this accumulated knowledge did not translate into therapeutic changes until the late 1800s (Porter GBM 76-77; Wooton 6, 16).

Rather than taking the holistic perspective of Hippocrates, Galen's teachings focused on the elemental components that form the human body; over time, this led to a reductionist, standardized, objectified view of patients (Eskinazi 1621-1622; Kleinman 22; Lock 121-122; Mishler 17; Porter, Blood and Guts 55). Like Hippocartes, Galen believed in the power of observation, but considered medicine a kind of intellectual puzzle; the primary focus of medicine was to accumulate knowledge, rather than heal patients. Continued scientific advances have allowed researchers and practitioners to examine the human body in enhanced detail, focussing an increasingly microscopic gaze on cells, molecules, and now genes. As Porter states:

The emergence of this high-tech scientific medicine may be a prime example of what William Blake denounced as 'single vision', the kind of myopia which (literally and metaphorically) comes from looking doggedly down a microscope. Single vision has its limitations in explaining the human condition... (GBM 8). The taxonomies of anatomy and disease derived from this research model have led to a sense that biomedicine is both universal and neutral, a rather myopic perspective that tends to eliminate non-Western or "alternative" medical traditions, as well as dismissing the flesh-and-blood patient in preference for data (Beck 23; Lock 121; Lock and Nguyyen 17; Mishler 15, 196; Worsely 315). While this tightening of focus legitimizes biomedicine as a scientific undertaking, it simultaneously objectifies the living patient, rendering it increasingly difficult for physicians to treat patients holistically or retain a humanistic medical practice.

At the extreme, the biomedical enterprise seems to overwhelm all other perspectives, creating a "medicalization of life" in which normal biological events, such as birth and death, 
become conditions requiring medical management (Illich 11). Medical Nemesis: The Expropriation of Health, philosopher and critic Ivan Illich's 1975 dystopian portrait of contemporary medicine, represents what might seem an extreme view, opening with the declaration that: "The medical establishment has become a major threat to health. Dependence on professional health care affects all social relations" (11). Substantiated by statistics, there can be no doubt that biomedicine is not always effective, and that there are increasing problems with iatrogenic conditions, illnesses caused by physicians or the clinical context. ${ }^{13}$ Illich writes: "In a complex technological hospital, negligence becomes 'random human error,' callousness becomes 'scientific detachment,' and incompetence becomes a 'lack of specialized equipment.' The depersonalization of diagnosis and therapy has turned malpractice from an ethical into a technical problem" (24-25). Supported by a medical system that seems to prize corporate profit and political power above individual welfare, his conclusions are not unreasonable. Illich also contends, however, that using biomedicine for profit or power is a misappropriation (11). Biomedicine, in its purest form, can lead to ethical quagmires like Willard Gaylin's "neomorts" - newly dead retained on life support in "bioemporion" to provide raw material for medical students and researchers (26). Adele Clarke et al. echo Illich's sentiments, moving them into the new millennium by noting that medicalization has become biomedicalization, with "The extension of medical jurisdiction over health itself (in addition to illness, disease, and injury) and the commodification of health..." (162). For the sake of this dissertation, the focus will not be on corporate profit or institutional power-although these are substantial issues in contemporary healthcare - but rather on beneficent uses of biomedicine in the, perhaps naive or contentious, assumption that medicine is designed to aid patients and improve their health. Issues of

\footnotetext{
${ }^{13}$ A prime example is nosocomial or hospital-acquired infections, such as C. difficile, that are often resistant to antibiotics and frequently fatal.
} 
individual power are inescapable in the patient-physician dynamic and will be examined in this study.

\section{From Medieval to Renaissance Anatomy: Deconstructing the Body}

And if you are attracted to such a thing you will perhaps be prevented by the stomach, and if this does not prevent you, it will be the fear of spending the hours of the night in the company of such bodies, quartered and skinned and unpleasant to see.

Knowledge of the human body improved with anatomical studies, carried out with varying degrees of success and secrecy, depending on time, place, socio-political, and religious constraints. While it can be argued that successful patient treatment requires bodily knowledge, and that therefore anatomical work benefitted patients, it can also be argued that most anatomists were only interested in the pursuit of scientific knowledge, and that anatomy had no obvious benefit to living patients for centuries. Galen's innaccurate anatomical conclusions, noted in a dozen anatomical texts of his corpus, endured until the 1500s, when Vesalius discovered that they had been rendered from primates and other non-human animals (Aufderheide 5; Porter, GBM 75, 179; Wootton 76; Vesalius, On the Fabric of the Human Body v). Galen's practice was subject to laws prohibiting the dissection of cadavers due to the perceived sanctity of the human body. Even after the fall of Rome, however, Galen's errors went unchecked for centuries, largely due to Church edicts. ${ }^{14}$

\footnotetext{
${ }^{14}$ During the early Medieval period, monks and monestries played an integral part in medicine, compiling new Latin texts on the care of the sick, as well as prescribing herbal remedies derived from their own monestry gardens. This period also saw increased belief that "disease was either punishment for sins, posession by the devil, or the results of witchcraft," for which the appropriate cure was prayer, rather than physicial intervention (Ackerknecht 83). In 1130,
} 
The shift away from ecclectiastical-based medical practice allowed the development of scholastic medicine at various newly founded universities. ${ }^{15}$ Although most early medical professors were also clergymen, university medicine was less influenced by the Church and more by Ancient Greek and Arab texts ${ }^{16}$ (Ackerknecht 87, 84; Wootton 50-51). The wisdom of these texts was accepted without question, supporting the persistence of earlier errors. Medicine became a text-based philosophical pursuit, undertaken in libraries, rather than with patients, so there were limited opportunities for empirical research (Ackerknecht 89; Porter, GBM 114). University medicine also initiated the professionalization of medicine through a set curriculum, the granting of the title "doctor," state licensing exams, and the development of guilds for medical apprenticeship (now referred to as internship) (Ackerknecht 92-93). Such highly educated doctors were few in number ${ }^{17}$ and only employed by society's elite; the majority of

the Council of Clermont forbade clergymen from practicing medicine, fearing that such earthly practices diluted their religious focus (Ackerknecht 84; Johnson 172; Pruitt 715). Thirty-three years later, in 1163, the Council of Tours drafted the Ecclesia Abhorret a Sanguine, forbidding clergymen from shedding blood, an edict reissued by Pope Innocent II in 1215 with the intent of stopping all surgical and anatomical activities (Ackerknecht 89; Johnson 172; Pruitt 715-716). Church edicts against clerical bloodshed resulted in a split between medicine and surgery; while monestries retained their herb gardens, surgery (and bloodletting) was relinquished to barbers, hangmen, animal gelders, and untrained laymen, where it remained for nearly seven centuries (Ackerknecht 89). During his Papacy from 1198-1216, Pope Innocent III issued a further Ecclesia Abhorret a Sanguine intended to end all surgical activity (Pruitt 716). Innocent III did, however, allow forensic post-mortems to be conducted in the event of a suspicious or untimely demise; during the Black Death in 1349, dissections were practiced for medico-legal reasons in some regions of Italy and France (specifically Florence, Montpellier, and Avignon) (Ackerknecht 91; Ongaro 154; Porter, CHM 137). Around 1300, Pope Boniface VIII decreed that the dismemberment and de-fleshing of Crusaders' bodies, to enable the transportation of their bones home, must cease (Aufderheide 5; Porter, GBM 132; Wootton 51). Such edicts were generally perceived as prohibitions against anatomical research. Porter notes that in the late-Medieval period, some countries began legalizing dissection for educational purposes. In 1482, Pope Sixtus IV decreed that bodies could be dissected if they were those of executed criminals and the remains were later buried. In this way, dissection was viewed as a continuation of earthly punishment for crimes committed, a trend some see as continuing into the Visible Human Project (Porter, BG 54; Porter, GBM 132; van Dijck 127; Waldby 13-15).

${ }^{15}$ Noted medical schools were established at the University of Paris (1110), the University of Bologna (1158), Oxford University (1167), the University of Montpellier (1181), Cambridge University (1209), the University of Padua (1222), and the University of Naples (1224) (Porter GBM 113).

${ }^{16}$ Medieval and early Renaissance European medical education was dominated by the Hippocratic corpus, the Galenic corpus, and the Kitah al-Qanum or Canon of Arab medical scholar Abu Ali al-Huaayn ibn 'Abdallah ibn Sina, known in Latin as Avicenna (980-1037). The Canon was a compilation of all medical knowledge, from the Ancient Greeks, to the Alexandrians and Arabs (Ongaro 156; Porter GBM 98).

${ }^{17}$ The completion of a medical degree required ten years, so few students completed their studies. The highly respected University of Bologna graduated only sixty-five doctors in the fifteen years between 1419 and 1434 
patients self-diagnosed and self-medicated, or consulted barber-surgeons and other lay

practitioners. In extreme cases, the very sick or older poor might turn to hosptials, but often these only offered food and shelter and were essentially places to die (Ackerknecht 93; Porter, $C H M$ 56-57).

Medieval and Renaissance Europe had a two-tiered medical system; educated physicians (for serious illnesses) and trained pharmacists (for everyday needs) tended to the urban wealthy, while the urban poor and rural peasants consulted laypeople. Well-educated doctors diagnosed their elite patients solely from urine samples, altogether dismissing the need for patientpractitioner encounters (Wootton 63). By the mid-1500s, Venice, for example, boasted over fifty pharmacies, one for every 3,000 residents, selling remedies from bezoars to combat poisoning, to Egyptian mumia, a cure for every conceivable ailment (Eamon 161). The pharmaceutical trade in Venice relied heavily on the ritualistic production, sale, and export of theriac, a Galenic panacea. Venetian theriac was reputed to be the best, as it contained sixty-four ingredients; as well as the traditional opium and honey, it also included numerous herbal ingredients, and uniquely, the flesh from vipers captured in the Euganean Hills near Padua ${ }^{18}$ (Eamon 163). In rural Italy, peasants turned to streghe (witches), wise women experienced in matters of birth, illness, and death who treated patients with locally grown herbs, often combined with amulets and the unsanctioned use of prayers. Remedies were simple, often containing only two or three ingredients; for instance, a small bag of rue and lavender worn around the neck protected against illness, even the plague (Magliocco n.p. $)^{19}$.

(Porter GBM 114). Ackerknecht cites that in 1296, Paris had only six doctors; by 1395, they could boast thirty-two (93).

${ }^{18}$ So many horned vipers were captured in the Euganean Hills for traditional theriac production, that the snake was extinct by the $17^{\text {th }}$ century (Eamon 164).

${ }^{19}$ For further details, see my paper, "Curing Venice's Plagues: Pharmacology and Witchcraft" forthcoming in a special issue of postmedieval on Medievalism and the Medical Humanities in 2017 (volume 8.2). 
Owing to the combination of Church control and academic pursuit, medicine made few practical advances for centuries. As evidenced by one of the first recorded public autopsies by Mondino de Luzzi (also known as Mundinus, c.1270-1326) in Bolgna c.1315, anatomy remained unchanged. The drawings in his Anatomia corporis humani (written in 1316 but not published until $1478^{20}$ ) are profoundly lacking in anatomical detail, and more closely resemble a child's Hallowe'en project than a serious anatomical endeavour (Porter, GBM 132). Although these illustrations purport to be based on Mondino's own observations, they repeat a number of inaccuracies introduced by Galen and other Ancients. For instance, Mondino reports that the heart is tri-ventricular (rather than four-chambered as it is understood in modern anatomy), a notion from Artistotle, supported by Galen (Van Praagh and Van Praagh 462). Mondino also discusses the five-lobed liver (rather than the four lobes understood by modern anatomy), again reflecting Galen (Porter, GBM 132). Clearly the ghost of Galen was more influential than Mondino's own observations; however, medicine soon began to evolve in Renaissance Italy. ${ }^{21}$ Whether this reinvigoration of medical science aided patients of the period is questionable, which is not to say that patients were entirely ignored by physicians or advances in medical science.

\footnotetext{
${ }^{20}$ The publication delay may have been due to the Church's continuing ban on human dissection. This ban was finally lifted by Pope Sixtus IV in 1482, four years after Mondino's posthumous publication (Porter GBM 132)

${ }^{21}$ Leonardo Da Vinci, a man of both science and art, completed 120 pages of notated drawings towards an anatomical atlas. With the Church ban against anatomy lifted, he took instruction from Marcantonio della Torre at the University of Pavia in 1507, and was granted access to various hospitals to continue his research until 1515. At the Hospital of Santo Spirito in Rome, his Church-appointed assistant reported his activities to the Vatican, and Da Vinci was brought before Pope Leo X on charges of witchcraft and necromancy. Ordered to cease and desist, or face dire consequences, Da Vinci abandoned the project. The pages passed through various hands and were eventually procured by the British Royal Collection in 1630; they were not published until the twentieth century, after many of Da Vinci's findings had been rediscovered by others. Da Vinci's drawings were unique for the period: they were not influenced by Galen, but based on his own careful observations as a visual artist; they were rendered from several corpses, creating generalized anatomical norms; and they were drawn from several angles, creating a sense of threedimensionality. Da Vinci's carefully rendered drawings and notes reveal accurate details of pregnancy and foetal development, as well as observations about arteriosclerosis with age (Pedretti and Frost; Porter, GBM 177 ). These are findings that later proved helpful to patients; however, they were not publicized due to Church pressure. Clearly the lifting of the dissection ban had limits.
} 
Anatomist Andreas Vesalius (1514-1564) began his often-overlooked Bloodletting Letter (1539) by wishing "Health unto suffering man" and several times he acknowledges the pain patients experience $(37,58)$. Throughout this lengthy letter, Vesalius's tone vascilates between that of the respectful young student bowing unctuously to the mastery of Galen, and the precocious young student frustrated by Galen's failure to recognize the obvious-yet still worried about the reception his own controversial ideas will receive (BL 44, 60, 82-83). Vesalius's interpretations of observable findings often differed from Galen's; as will be discussed in the next chapter, the interpretation of the observable continues to be problematic in medical imaging. Vesalius was clearly frustrated that a slavish respect for tradition impeded the development of medical knowledge, and he called for a more practical approach to solve scientific problems and provide better patient treatment (BL 33-34). He considered anatomy the path to the reunification of medicine and surgery, stating: “...it behooves each and every physician to observe these things from the anatomy of bodies and not like our Aescalapians, relying solely on a pile of authorities and without exact knolwedge of the veins to be sectioned in disease..." (BL 5). Recalling Galen's primacy of observation for scientific discovery, Vesalius used the master's methodology to effectively undermine the master.

Vesalius is generally considered to have revolutionized human anatomy, and is sometimes referred to as the founder of modern medicine. Upon receiving his doctorate at the age of twenty-three, in 1537 he was appointed Chair of Surgery and Anatomy at the University of Padua, the school closest to Venice and an institutional breakway from the more conservative University of Bologna (Vesalius, $F H B$ xix). Prior to Vesalius, anatomical instruction required a teaching team: a lowly surgeon did the actual dissection; a lofty physician, or "extraordinary" professor, sat in a throne-like chair reading aloud from Galen's work on anatomical procedure, or 
Mondino's more recent work still reflecting Galen's errors; and an "ordinary" physician, or teaching assistant, explained the relationship of the text to the cadaver ${ }^{22}$ (Ongaro 160; Porter, GBM 133). The young Vesalius ignored this tradition, got down from the chair, and bloodied his hands $^{23}$ (Ongaro 164, 168; Reiser, Medicine and the Reign of Technology 13; Vesalius, FHB xix). Engaging with cadavers in this way, trusting his own observations, allowed Vesalius to rewrite Galen, overturning one-and-a-half millenia of entrenched medical thought. In Foucault's words, anatomists shifted the gaze from the body's surface to its interior, "plunging from the manifest to the hidden" (Birth of the Clinic 166). It was Vesalius who first recognized that Galen's anatomy was derived from animal rather than human bodies; Vesalius discovered that the human heart had four chambers, rather than the traditional three (Ongaro 167; Vesalius, $F H B$ $\mathrm{ix}, \mathrm{v}, \mathrm{vi}, \mathrm{xx})$.

Medical illustrator Shelley Wall marks Vesalius as "the first to insist on the primacy of visual documentation" (Wall 136); he was the first professor of medicine to incorporate visual aids into his lectures, creating the Tabulae anatomicae sex (Six Anatomical Tables), published in 1538 (Ongaro 167; Vesalius, FHB xix). In 1543, Vesalius published De humani corporis fabrica libri septem (On the fabric of the human body in seven books), the first fully illustrated and detailed anatomical atlas. ${ }^{24}$ In addition to a Latin ${ }^{25}$ text in excess of 80,000 words, the volumes

\footnotetext{
${ }^{22}$ This seems a rather challenging position for a T.A., as the text and the cadaver were not always in agreement. The position would have required a certain imagination.

${ }^{23}$ Most anatomical cadavers were executed by hanging, or removed from graves, so the bodies were usually in a gruesome condition; add to this the lack of electricity for either refrigeration or lighting. In Vesalius's time, dissections were performed only in winter, in temporarily constructed outdoor anatomy theatres for public, as well as academic, audiences. The famous anatomy theatre at the University of Padua was not built until 1594, after Vesalius's tenure. The first of its kind, and model for all that came later, this torch-lit theatre could accommodate 300 students, standing in raised concentric rings behind high railings to prevent accidental falls in the event of fainting (Bonati n.p.).

${ }^{24}$ It must be noted that Johannes Gutenberg's printing press was invented in 1440. This new technology enabled mass printing and wide distribution of the Fabrica.

Arguably the next (and last) successful anatomical atlas prior to contemporary imaging was Henry Gray's Anatomy: Descriptive and Surgical (1858) illustrated with 1247 engravings by Henry Vandyke Carter.
} 
also contain over $300^{26}$ images $^{27}$ of both complete systems and their details, with over 900 alphanumerically coded markers linking diagrams to text (Ongaro 168; Vesalius, $H C F$; Vesalius, $F H B$ Xxxv-Xxxvi). The images engraved by Jan Stephen van Calcar (c. 1500-1546), ${ }^{28}$ and students of the school of Titian, were overseen by Vesalius himself. While an impressive book, the Fabrica, like other anatomical atlases, effectively flattens the three-dimensional corporeal body, creating a map of the human territory for physicians to explore. Vesalius's revisions to Galen, ${ }^{29}$ undertaken in the name of accurate science, prompted merciless attacks from his peers (Vesalius, $F H B \mathrm{xx}$ ); even Sylvius, Vesalius's former professor at the University of Paris, renamed him vesanus, or madman (Porter, GBM 179). Simultaneously, the reinvigoration of first-hand observation launched a new and powerful direction for scientific medicine, ${ }^{30}$ paving the way for modern medicine and biotechnology, including diagnostic imaging.

Given our earlier definition of biology as "the study of living organisms," it seems contradictory that so much of Western scientific biomedicine is based not on the living, but on dead organisms (Leder 17; Porter, CHM 9; Porter, GBM 8; Waldby 39). Sociologist Catherine Waldby notes that, "Medicine relies upon productive encounters with corpses...the medical idea

\footnotetext{
${ }^{25}$ While most of the text is Latin, occasionally Greek is also used. On two pages, text appears in Latin, Greek, and Hebrew, presumably to allow comparison of words denoting specific anatomical features (Vesalius, HCF 166-167).

${ }^{26}$ This is Giuseppe Ongaro's number, but not all historians agree (168). As well as the anatomical prints, each section of the Fabrica opens with an illuminated letter featuring putto doing unlikely things, like grave-robbing, practicing necromancy, dissecting corpses, removing a body from the gallows, vivisecting a dog, and defecating (this last image was removed from later editions). The discrepancy in the number of plates may be due to the inclusion or exclusion of these purely decorative graphics (Vesalius $H C F$ ).

${ }^{27}$ While most of these images are headless, limbless, and anonymous, the notable exceptions appear in Book VII's renderings of the brain. Here, the skull is opened to reveal the brain, while the cadavers' faces are clearly visible, in some cases with scalp and facial hair (Vesalius HCF 605-609).

${ }^{28}$ Though some historians contend that these illustrations were the work of Van Calcar, a friend of Vesalius's, others question this assertion (Ongaro 167; Vesalius $F H B \mathrm{xx}$ ).

${ }^{29}$ The index of Fabrica reveals only eight references to Hippocrates, but eight columns of references to Galen! (Vesalius $H C F$ )

${ }^{30}$ After Vesalius, the University of Padua supported the anatomical work of many notable scholars. Among them were Realdo Colombo of Cremon (1516-1559) who advanced cardiac work; Gabriele Falloppio (1523-1562) who explored embryology and proposed the idea of tissues beyond gross anatomy; Fabricius ab Acquapendenta (15331619) who advanced work on embryology and sense organs; and William Harvey (1578-1657) who proposed the closed circulatory system (Ongaro 168-171, 179-181).
} 
of life is haunted by the corpse" (23). Although this is not something most patients consider when entering their doctors' offices, contemporary medical education still considers cadaveric anatomy a necessary rite of passage (Cappabianca and Magro 597; Dyer and Thorndike 974-979; Gregory and Cole 1181; Leder 22; Roosen et al. 562). Porter notes that, initiated by dissection, biomedicine, "has sustained the fruitful conviction that in ever-more-minute investigation of the flesh lies the key to health and disease, even if that has also encouraged a tendency to myopic reductionism, to miss the whole by concentrating exclusively upon the parts" ( $B G 55)$. While anatomical history is strangely fascinating, it leaves the living patient glaringly absent. Without much imagination, one can readily fill this gap with the silence of cadavers, the listless passing of the diseased, the screaming of the wounded, and the wailing of mothers in this period before vaccines, ${ }^{31}$ antibiotics, anaesthesia, ${ }^{32}$ and knowledge of disease transmission. ${ }^{33}$ Rather than having a patient-centred focus, the line of continuity running from anatomy towards contemporary biomedicine is that of the Cartesian "clockwork man," a model that portrays the body as a mechanism. ${ }^{34}$ Given Kepler and Galileo's advances with mechanical astonomical models during the 1600s, it is hardly surprising that René Descartes might apply a

\footnotetext{
${ }^{31}$ The first vaccine was created by Louis Pasteur in 1881 for anthrax; others soon followed (Wootton 20).

${ }^{32}$ Although nitrous oxide was found to be a successful anaesthetic in 1795, the first application of anaesthesia to human patients was by Horace Wells, a dentist in Rochester, N.Y. in 1824. The European reception of "the Yankee dodge" was so hostile that Wells committed suicide. Anaesthesia was not used in Europe until 1846 (Wootton 22).

${ }^{33}$ Noticing that the rate of childbed (puerperal) fever in the ward tended by medical students was nearly ten times higher than the ward tended by midwifery students, Dr. Ignaz Semmelweis (1818-65), of the Vienna General Hospital, realized that the medical students were coming directly from the morgue to deliver babies, thus introducing germs to vulnerable mothers and infants. Once Semmelweis instituted chlorinated-water hand-washing prior to deliveries, mortality rates dropped radically. His ideas were dismissed by his peers, causing him to resign (Porter, GBM 369-370). Florence Nightingale spearheaded the effort to reduce the hospital death rate from $40 \%$ to $2 \%$ within six months at the hospital at Sebastopol during the Crimean War (1853-56), an improvement accomplished largely through improved hygiene (Porter, $B G$ 147). This dramatic change was noticed on the home front, and cleansers were in use in hospitals by the late-1800s thanks to Joseph Lister (1827-1912); Louis Pasteur started sterilizing medical instruments in 1874; and rubber gloves were invented in 1890 by William Halsted to protect his nurse-wife's hands from harsh antiseptics (Porter, CHM 199).

${ }^{34}$ It should be noted that mechanistic fascination and the use of clockwork or mechanics to explain human animation did not begin or end with Descartes; his is simply the name most readily associated with the idea (Dear $58,60,67)$. Also noteworthy is that, similar to Galen, Descartes prized reason over passion, considering the latter to be an unbalanced aberration (Dear 69; Wootton 40).
} 
similar mechanical explanation to humans, effectively distancing physicians from their patients and patients from their own bodies (Osherson and AmaraSigham 221-222). In his Discourse on Method, for instance, Descartes describes the movement of the heart, saying that it, "follows just as necessarily as the movement of a clock follows from the force, position, and shape of its counterweights and wheels" (Descartes cited in Dear 59). This is the previously noted reductionist view, a faith that digging ever-deeper, with more advanced technologies, will eventually reveal what makes the human mechanism tick. In the 1640s, Descartes conceived a dualistic ontology, a split between the res extensa (body) and the res cogitans (mind or soul), espousing a mechanistic view in which the mind is superior to the corpus, declaring the mind as the seat of conscious identity in his often-quoted phrase, cogito ergo sum (I think, therefore I am). The effect of this split on medicine was that, as Porter so deftly puts it, "wellbeing became compared to the running of a well-tuned, well-oiled machine, and sickness was depicted as a mechanical breakdown, due perhaps to a blockage, fuel shortage, or excessive friction" ( $\mathrm{CHM}$ $81)$.

This reductionist Cartesian binary split continues to influence many aspects of Western culture, including medicine. Psychiatrist Leon Eisenberg asserts that, "The dysfunctional consequences of the Cartesian dichotomy have been enhanced by the power of biomedical technology" (9). Eisenberg, Arthur Kleinman, and others, make the distinction between the medical focus on disease, and the patient's focus on the illness experience, underlining that disease can be diagnosed without an experience of illness (e.g., high blood pressure, some cancers), that a patient may experience illness without a diagnosable disease (e.g., psychosomatic symptoms), and that notions of disease and illness are culturally variable (Eisenberg 11-12; Morris 71). Dominant biotechnology focuses on the minutiae and mechanics of disease, often 
considered in fragmented and isolated sections of the body, rather than the patient's experience of illness; in Cartesian terms, biotechnology focuses on the mechanism of the body, rather than the abstraction of the mind or soul. Surgery requires a conception of a body separate from the mind; organ transplantation requires the conception of a fragmented body with interchangeable parts (Brown and Webster 107-108; Osherson and AmaraSigham 226; Poole et al.; Rose 21). Psychologist Samuel Osherson and anthropologist Lorna AmaraSingham observe that even the contemporary medical management of death relies on a mechanical model of the human body (221); in a litigious cultural environment, dominated by technology, the quantity of life seems more highly prized than the quality of life.

This dualistic mindset is also necessary for the mechanistic separation of data from bodies, whether for routine blood testing and diagnostic imaging, or for the creation of the Human Genome Project (HGP), and the complex imaging of the Visible Human Project $(\mathrm{VHP})^{35}$. At the extreme, one might perceive that the romantic notion of a visceral, organic human body has been superseded by the idea of cold, hard data. This focus on the body as a data source promotes the res extensa as the central focus of the human project, rather than Descartes's preferred res cogitans (Porter, GBM 248; Rose 254). Cartesian dualism supports the objectification of the body; it effectively "detaches a body from a person" (van Dijck 11). As physician-philosopher Drew Leder asserts, however, "The full significance of human disease and health necessarily eludes the model of body-as-machine," and yet this remains the dominant model in biomedicine (31). What is missed, and its relevance, is one of the primary concerns of this dissertation.

\footnotetext{
${ }^{35}$ The Visible Human project is an interactive online anatomical archive rendered from single individuals using a combination of MRI, CT, and digital photography.
} 


\section{The Modern Era: Professionalized Doctors and Public Health}

We, the undersigned Doctors of medicine of the Faculty of Paris, certify to all to whom it may concern, that the qualities of antimony ${ }^{36}$ are recognized by us to be very useful for the cure of a number of illnesses. We certify this on the basis of long usage and continued experience. Further we declare that this remedy which has for so long been charged with having a poisonous malignity has many rare virtues and that a physician can successfully employ it to combat a great number of diseases provided that he uses it with a prudence and discretion.

Declaration signed by sixty-one members of the Parisian Medical Faculty March 26, 1652

Eusébe Renaudot (1653) in Allen G. Debus The French Paracelsians (97)

The sciences and medicine became more codified and professionalized in the eighteenth and ninteenth centuries. New sciences of biology and chemistry expanded as various learned men conducted experiments on living organisms ${ }^{37}$ (Porter, GBM 253). Although Henry VIII had chartered the College of Physicians as the medical licensing and policing body for London in 1518, The British Medical Association was not founded until 1832 (Porter, GBM 198; Ackerknecht 218). Only a few years later, in 1847, the American Medical Association was established, followed twenty years later by the Canadian Medical Association (Ackerknecht 218; CMA). While earlier journals, such as the British Gentleman's Magazine, founded in 1731, offered a cross-pollination of ideas between physicians and the public (albeit a public of a certain education, class, and gender), professional associations were quick to create their own medical journals that were the exclusive domain of physicians (Porter, PP 283-314). Latin, already

\footnotetext{
${ }^{36}$ Antimony is a metal; according to the American Environmental Protection Agency (EPA), ingestion causes gastrointestinal problems, while inhalation can cause skin and eye irritation, as well as lung and heart problems. Long-term or high exposure may cause cancer. In short, antinomy is now considered highly toxic (EPA).

${ }^{37}$ These included better understanding of digestion and respiration, as well as experimenting with the effects of gases, such as nitrous oxide (Porter GBM 253-254).
} 
commonly used in medical and other educational texts, expanded into a new professional language that largely excluded the laity. Journals still currently in publication, including The Lancet (1823), the British Medical Journal (BMJ, $\left.1840^{38}\right)$, and the Journal of the American Medical Association (JAMA, 1883), supported the professionalization of medicine by establishing sites for formalized medical discourse (Porter, GBM 351, 354; BMJ; JAMA). The Canadian Medical Association Journal (CMAJ) was not founded until 1911, by which time a professional medical language was already well established (CMA).

In this era of nascent professionalization, competing theories of disease transmission emerged; was disease transmitted through miasma (bad air) or was contagion responsible (Porter, GBM 259; Wootton 180)? This period was also marked by increased industrialization and urbanization, creating the problem of a growing poor population, often living in squalid conditions. This hotbed of disease subsequently led to the concepts of public sanitation and the public good. Improved sanitation did more to promote general health by effectively preventing disease, than available medical treatments could offer (Wooton 278-281). ${ }^{39}$

\footnotetext{
${ }^{38}$ Porter suggests 1857 as the first date of publication, however, the $B M J$ website gives $1840(B M J)$.

${ }^{39}$ For example, before reliable sewage control, outbreaks of cholera, a waterborne bacterial infection affecting the intestines, were common, especially among the poor. Symptoms included vomiting and diarrhea, and the disease was often fatal. London physician John Snow's published findings (1849), establishing that cholera was transmitted through infected water, were generally ignored. In the summer of 1854, Snow discovered that houses whose water was supplied by the Southwark and Vauxhall Company had 286 cholera fatalities, whereas houses in the same neighbourhoods supplied by the Lambeth Company had only fourteen cholera deaths. The difference was that Southwark and Vauxhall drew Thames water near sites into which London's sewers emptied; Lambeth drew Thames water further from obvious contamination (Wootton 201-203). Snow's most dramatic breakthrough occured when, after about 100 deaths in the same London neighbourhood within ten days, he asked the local authority to remove the Broad Street communal pump handle, preventing water access; twenty-four hours later, the outbreak was over (Porter, GBM 413; Wootton 204-205). Snow published a revised and expanded version of his findings in 1855; however, in spite of Snow's carefully compiled data, his theory of contagion and disease transmission was not generally accepted for at least another decade (Wootton 195). Even after his formal presentation to parliament, agreement to build the necessary sewage infrastructure did not occur until after "the great stink" during the summer of 1858, and construction was not completed until 1875 (Porter, GBM 413). A Medical Department was established in London, giving local authorities powers to reduce industrial pollution and provide clean water to tennants. In 1867, London's Medical Department passed the Vaccination Act, compelling parents to vaccinate their children, and establishing the policing of public health (Porter, GBM 414).
} 
Prior to an understanding of its transmission and prevention, physicians thought that cholera was caused by miasma ${ }^{40}$; hence, prevention was ineffective and recommended treatments were frequently harmful to patients. Accoring to a July 25, 1832 letter from one J. Pidduck, M.D. of London, cholera treatment involved: keeping the patient warm and in bed; the hourly administration of a tablespoon of salt in water to promote vomiting and perspiration; offering the patient thin gruel, soda, or plain water; the optional application of leeches to the stomach and behind the ear to quell abdominal and head pain; in the event of fever, the adminstration of medications, including calomel pills (mercury chloride [Porter, GBM 266] combined with powdered rhubarb), castor oil, a cordial including jalap (morning glory) and sweet spirits of nitre $^{41}$ (ethyl nitrite), and a saline mixture including carbonate of potash and carbonate of soda to promote additional vomiting; stimulants, such as brandy, and astringents, like laudanum, which might reduce vommiting, were to be avoided; certain indigestable foods and strong emotions, such as fear, should similarly be avoided; in addition to the afforementioned leeches, bleeding might also be necessary (Every Man His Own Doctor 5-7 ${ }^{42}$ ). Dr. Pidduck's complex remedy compelled further vomiting and perspiration in a disease-state that already causes vomiting and diarrhea, potentially dehydrating the patient even more quickly. Additionally, while rehydrating the patient with water seems appropriate, if that water was from the same contaminated well, the

\footnotetext{
${ }^{40}$ Alain Corbin's The Foul and the Fragrant: Odor and the French Social Imagination (1986), documents the amalgamated scents that polluted the urban landscape during this period, based, in part, on the catalogue of stenches accumulated by Jean-Noël Hallé, the first chair of public hygiene in Paris in 1794. The combined smells given off by the Seine included human and animal excrement, blood, urine and other bodily fluids, mud, offal, corpses, and body parts discarded by medical students, creating an acidic, sulphurous-smelling soup that caused nausea, burned the eyes and throat, and was rumoured to be toxic (1-4). Given "the great stink" suffered by Londoners in the summer of 1858 , the condition of the Thames was probably similar.

${ }^{41}$ In rural Ontario in the 1920s, my mother was prescribed sweet spirits of nitre for pyelitis, a kidney infection (Jenkins, I. 27). Antibiotics, which would be used to treat this condition now, were not yet available. Medications from the eighteenth and nineteenth centuries were still in use well into the twentieth century, especially in rural areas, in spite of their questionable efficacy.

42 This small volume, reprinted from 1835, is crammed with fascinating remedies for a variety of human and animal ills, as well as handy household tips. Some ingredients are no longer commercially available, or are no longer known by their archaic names; others are now known to be ineffective or poisonous. The final entry regards the application of electricity, a powerful remedy for anything from toothache to deafness.
} 
patient would be continually exposed to cholera bacterium. Mercury chloride, once thought to cure syphillus and a variety of other ills, is no longer prescribed, as mercury is now recognized as lethal (Porter, GBM 175). Although well intentioned, Dr. Pidduck's remedy, like many others of the era, may have actually increased the likelihood, speed, and discomfort of the patient's demise $^{43}$. Snow's findings regarding germ theory were eventually accepted, laying the foundation for other germ discoveries (e.g., typhoid, typhus, tuberculosis, leprosy, and smallpox) and modes of prevention (Porter, GBM 413). While cholera still exists, it is now treated with antibiotics and rehydration (provided such interventions are available in the disease context).

As well as improving sanitation, notions of the public good also expanded to the changing role of hospitals. Although in existence in Europe since about 450, hospitals were charitable Christian institutions offering food and shelter to the poor, with limited capacity for caring for the sick, the dying, the homeless, or the mad (Porter, CHM 56-57). The Hôtel-Dieu ${ }^{44}$ in Paris, for instance, was established in 651 and run by religious orders until the French Revolution in 1789 (Porter, $B G$ 137). The Hôtel-Dieu provided ample opportunities for honing the surgical skills of a young physician, Marie François Xavier Bichat ${ }^{45}$ (1771-1802), who was studying in Lyon and Paris during the Terror of 1793-94 (Porter, GBM 265). Documenting his post-mortem efforts and observations during his tenure at the Hôtel-Dieu, Bichat published Traité des Membranes (Treatise on Membranes) in 1799, revolutionizing pathology by theorizing that diseases occurred in tissues (cells, nerves, arteries, etc.), rather than organs, and

\footnotetext{
${ }^{43}$ Dr Pidduck's is the kind of ministration that prompted George Bernard Shaw to pen The Doctor's Dilemma (1909), in which physicians grow rich by performing unnecessary treatments and surgeries, with little or no evidence of their efficacy, on ill-informed patients.

${ }^{44}$ John Howard (1726-1790) inspected hospitals, as well as prisons, and declared the Hôtel-Dieu one of the worst he visited, citing that patients, some of them dying, were crammed five or six to a bed (Porter, GBM 297).

${ }^{45}$ A larger-than-life statue of Bichat by David D'Angers was erected at the entrance to René Descartes University, 12 rue de l'Ecole de Médecine in Paris in 1857. Tourist guides fail to mention the partially draped, flayed corpse that lies at the foot of the noble likeness of the young physician. A plaque, dedicated to both Bichat and his colleague Pierre-Joseph Desault, is displayed behind the admissions desk of the Hôtel-Dieu itself.
} 
paving the way for modern clinical medicine. Although Bichat's work was a leap forward, it did little to aid patients of the period.

Hospitals, once removed from Church control, helped secularize medicine and reunite surgery with medical practice. Even prior to the use of anaesthetics and antibiotics, surgeries were performed, initially on the battlefield or by barber-surgeons only on external lesions, but then in hospitals. The most common internal hospital surgeries were gynecological, with the first ovariotomy performed in 1809 . The mortality rate for such surgeries was between $18 \%$ and $25 \%$. While sometimes performed for legitimate medical reasons, "belly rippers" also undertook surgeries to relieve symptoms of hysteria or nymphomania (Porter, CHM 196-197; GBM 363). The St. Thomas operating theatre ${ }^{46}$ in London, offered such surgeries. Although associated with the ancient St. Thomas Hospital, the operating theatre was located in the adjoining spire of St. Thomas's Church. From 1822, this facility was used for women's operations; later, it became an emergency ward where rapid amputations were performed, with an estimated $40 \%$ mortality rate (Wootton 180). While the operating theatre's location may seem strange, it had two advantages; it was on the same floor as the hospital's women's ward, so patients could be wheeled in directly, and because it was located in a separate building, in this pre-anaesthetic era, patient screams were somewhat muffled (Old Operating Theatre Museum and Herb Garret).

Hospitals provided practitioners with larger population samples than those available through house visits. Doctors could improve their understanding of how diseases spread and perhaps how they could be prevented or treated; hospitals provided more opportunities to observe, and ultimately autopsy, more patients with the same diseases, thereby improving

\footnotetext{
${ }^{46}$ In 1862, the hospital moved and the operating theatre entrance was blocked off. The facility was rediscovered in 1956 by historian Raymond Russell and established as a medical history museum, with an entrance up the long, narrow spire steps. The operating theatre offers occasional lectures with simulated surgical demonstrations of period amputation techniques (Old Operating Theatre Museum and Herb Garret).
} 
diagnostic and pathology skills. This collection and interpretation of data by physicians began a new age of medical treatment, one in which: "The medicine of symptoms will gradually recede, until it finally disappears before the medicine of organs, sites, causes, before a clinic wholly ordered in accordance with pathological anatomy" (Foucault $B C$ 150). In other words, this period marked the establishment of the physician, his observations and interpretations, as dominant in medical discourse, while the patient's illness experience of disease symptoms, became secondary. Hospitals changed the relationship between physicians and patients. As sociologist Nicholas Jewson notes, during the preceding era of bedside medicine, the ill were viewed holistically and had the power to determine, in conjunction with the physician, the course of treatment $(240,227)$. Once hospitalized, however, the "sick-man" became the passive "patient" (Jewson 235); there was "a shift away from a person oriented toward an object oriented cosmology" and "The patient's interest in prognosis and therapy was eclipsed by the clinician's concern with diagnosis and pathology" (Jewson 231, 235). Wootton notes that with the rise of hospitals in France, patients were assigned to doctors according to their disease states, allowing that doctor to continue his research; physicians had complete control over medical decisions and patient complaints or non-compliance were not tolerated (178).

Medicine was removed from the Church, the bedside, and from academic theory and refocused on clinical observation, practice, and research (Ackernecht 146). As Ackernecht observes, however, "It is easy to sympathize with the fear felt by former generations at the prospect of 'going to the hospital.' There the internal patient died from 'hospital fever' (typhus), while the surgical patient mysteriously succumbed to "hospital gangrene"' (186-187). Until the discovery of sulfa drugs in 1935, and penicillin during the Second World War, physicians could provide little by way of disease treatment (Porter, CHM 133). 


\section{Michel Foucault: The Medical Gaze}

This new structure is indicated — but not, of course, exhausted—by the minute but decisive change, whereby the question: 'What is the matter with you?', with which the eighteenth-century dialogue between doctor and patient began (a dialogue possessing its own grammar and style), was replaced by that other question: 'Where does it hurt?', in which we recognize the operation of the clinic and the principle of its entire discourse.

\section{Michel Foucault, The Birth of the Clinic xxi}

Much medical humanities research relies on Foucault, specifically his concept of the "medical gaze" or the "clinical gaze," which lies beyond the simple act of seeing and includes all physician-gathered sensory data: the "sight/touch/hearing trinity" (Birth of the Clinic 9,103, 202). The clinical gaze includes not only observation, but also palpation, percussion, and auscultation upon the object of the patient's body. In its most reductive form, the clinical gaze causes patients to feel objectified; "in relation to that which he is suffering from, the patient is only an external fact; the medical reading must take him into account only to place him in parentheses" (BC 7). Foucault, however, also allowed for a more complex understanding, stating that the gaze, "establishes the individual in his irreducible quality" $(B C \mathrm{xv})$. In other words, the clinical gaze can simultaneously objectify and create the subjectivity of the patient; for Foucault, object and subject are not mutually exclusive, and can occupy the same spatial and temporal moment, much as Mol and Law have suggested. "The object of discourse may equally well be a subject, without the figures of objectivity being in any way altered" ( $B C \mathrm{xv})$. This dissertation attempts to explore the patient as both the object and subject of medical discourse. 
The Birth of the Clinic: An Archaeology of Medical Perception (1973) offers a critical look at the foundational perceptions and historical development of the patient-practitioner relationship, acknowledging, as has this chapter and works by Porter, Wootton, and others, its dominant biomedical bias. As detailed in the previous section, the Hôtel-Dieu offered Bichat, and other physicians, the opportunity to observe, and later autopsy, numerous patients with similar afflictions. This is the historical moment that causes Foucault to declare, "the great break in the history of Western medicine dates precisely from the moment clinical experience became the anatomo-clinical gaze" ( $B C$ 179). Regarding the impact of this shift on the patient-physician relationship, sociologist David Armstrong observes:

During Bedside Medicine the patient was in a position to dictate (and define) the nature of illness: hence the existence of a symptom-based medicine. After the advent of the hospital the doctor's dominant role ensured the emergence of a medicine based on pathological lesions which were inaccessible to the patient without medical interpretation (19).

The establishment of the clinic, or hospital, as a place where sick people congregated, and Bichat's subsequent 1799 publication, are, for Foucault, the pivotal historical moment that establishes the medical gaze: "With Bichat, the medical gaze pivots on itself and demands of death an account of life and disease..." (BC 179). While Bichat's work certainly marks a turning point, solidifying this gaze, other moments in medical history also contributed to the formation of the medical gaze we experience today.

This dissertation locates the estalishment of the medical gaze at an earlier historical moment: 1482, when Pope Sixtus IV first permitted human dissection for reasons of pure curiosity and education (Porter BG 54-57). This is the moment when the all-powerful Church 
finally shifted its stance on the sacred nature of the human body, allowing it to be fully objectified. This administrative shift grants Vesalius permission to freely practice anatomy and publish his groundbreaking work, De humani corporis fabrica libri septem, in 1543 . Waldby traces a similar line in The Visible Human Project, from Vesalius through to contemporary imaging technologies, noting, "The abolition of the bodily interior as private, or sacred, space, a process begun with the earliest systematic anatomies of the late Medieval period and extended with the abrupt application of $x$-rays to the body in 1895, take on a new vigour with the launch of Visible Human figures" (6). Commenting on Vesalius's early anatomical work, clinical professor Stanley Joel Reiser states: "The revolution that changed how doctors learned about illness and related to patients, and reversed their attitudes about actively exploring the body and using tools, began with and was vitally nourished by the study of the dead" (Technological Medicine 4). Supported by Waldby and Reiser's work, this dissertation argues that the medical perception of the patient as a flattened data set was initiated by anatomical atlases dating from the mid-1500s, prior to the establishment of modern medicine, and was only further entrenched by the institution of the clinic and biomedical technologies.

As the function of cadavers for anatomical exploration is purely objective, conceiving of anatomical atlases as the foundation for creating the medical gaze is perhaps more reductive than Foucault would like. While Vesalius's corpses were largely anonymous objects, Bichat argued for the need to observe both the living patient and his or her corpse. Observation, however, may or may not construct a subjective patient; Bichat was certainly not interested in the patient's subjective illness experience. For Bichat, the living patient offered "only a confusion of symptoms;" however, "Open a few bodies, this obscurity will soon disappear, which observation alone would never have been able to have dissipated" (Bichat in Reiser MRT 19). Death offers an 
end-point to the disease process, a logical perspective from which to draw conclusions. There is, however, no possiblity of a subjective patient emerging from a corpse, for a communicative subject who can share his or her illness experience. Bichat's corpses were just as objectified as Visaleus's. Anatomical atlases, with their construction of a flattened data set, centuries before the establishment of the clinic, seem a logical archeaological starting point for the medical gaze. The Birth of the Clinic makes clear that Foucault's focus was specifically on French medical history $(28,30,55)$. Although Padua is briefly mentioned, Vesalius is not ( $B C$ 68). While it should hardly be surprising that Foucault focused on Paris, with its available French archives, by doing so, he arguably omitted medical developments foundational to establishing the medical gaze predating the birth of the clinic.

Regardless of whether the foundational moment for the reductive objectifying medical gaze is the moment when the human body was offered for dissection, or Bichat's founding of modern clinical medicine, what is important is that the medical gaze became fixed on the inert corpse, rather than the living patient. As Waldby notes: "medicine relies upon productive encounters with corpses, donor cadavers, foetal tissue, and other forms of marginal life and nearlife...the medical idea of life is haunted by the corpse" (23). For living patients suffering through an experience of illness, this mapping of dead anatomy onto their living, breathing bodies can be, at very least, disturbing. Wall comments that, "anatomical knowledge derived from cadaveric dissection is extrapolated (and interpolated) back into the living body (Roberts 1996), but it is not an untroubled transfer" (136). How does medical practice transfer its objective gaze, honed on a cadaveric history, back into the living subjective patient?

The living patient is rendered corpse-like as he or she is objectified by the reductive clinical gaze. Leder observes that even for a routine physical exam, "the patient is placed in a 
position of corpse-like passivity" (22). Sociologist Mildred Blaxter contends that while patient $\mathrm{P}$ felt a sense of "responsibility" when undergoing diagnostic imaging, what she felt was "responsible for remaining absolutely still, following positioning instructions exactly" and "an enormous responsibility not to move, not to cough" (CVP 771). While these thoughts were noted and recorded by a subject, they clearly mark the actions (or inactions) of a subject endeavouring to be an object; the passive, docile patient, mimicking a corpse. Failure to do so would undermine the efficacy of the imaging technology. As Foucault observes, "It is when death became the concrete a priori of medical experience that death could detach itself from counternature and become embodied in the living bodies of individuals" (BC 243).

In the History of Madness (2006, orig. 1961), Foucault explores the ways in which the medical gaze impacts insane asylums patients, but these reflections are also helpful in unpacking patient experiences in contemporary medical hospitals. For instance, Foucault writes that, "Knowledge of sickness was...to be first of all an inventory of what was immediately manifest in perception, all that was most evident in truth. In this way medicine adopted, as its first approach, the symptomatic method" (HM 187). To perceive symptoms, the physician must observe the patient through a medical gaze. The patient's ailment (in this case madness), "became an object of investigation, a thing invested with language, a known reality: it became, in short, an object" (HM 443). According to this statement, it is the disease or disorder that is the object of the medical gaze, rather than the patient; the patient is merely a vessel containing the object of enquiry. The individual patient, as Foucault writes in The Birth of the Clinic, must be subtracted from this engagement (15). But, as Foucault notes, this objectifying subtraction of the individual implemented by the medical gaze, “...could only be accomplished with the complicity of the patients themselves" (HM 77). 
Although patients are, without doubt, complicit in this exercise of objectification, living patients also generally seek engagement; patients want to be seen, heard, and respected as subjects, not objects. Our sense of objectification in the presence of the medical gaze is commonly spoken of by patients saying that they feel like "a piece of meat" rather than a living human being (Leder 24). As Leder notes, "Reductionist aspects of the paradigm lead to reductionist modes of treatment" (3). Some theorists, philosopher Don Ihde for example, contend that Foucault's construction of the medical gaze, "simultaneously objectifies and enacts control over the selectively passive bodies" rendering "The body objectified by the medical gaze in the clinic" $(25,17)$. This purely reductive reading of the clinical gaze is the one most commonly associated with Foucault, but a more complex interpretation is possible.

A careful reading of Foucault shows that a reductive objectifying concept is only part of his notion of the medical gaze; it can also be creative. "The gaze is no longer reductive, it is, rather, that which establishes the individual in his irreducible quality $(B C \mathrm{xv})$ ". Interpreting the key phrase, "establishes the individual,” Armstrong writes:

The body is one such object that exists as a totally taken-for-granted phenomenon. It is therefore a radical step — and one that has been challenged — to argue that the body is created, or fabricated, or invented. And yet one only has to look to other systems of medicine, such as the humoral, to see parallels: could the skilled physician of the past identify humors which are currently beyond our perception, or was it a delusion, an error? (23)

Certainly, the patient established in humoural medicine differs from the patient established in contemporary Western medicine, just as the patient established in acupuncture differs from that established in chiropractic, etc. Each medical gaze establishes its own patient by virtue of 
observing specific markers or norms associated with that particular practice, training, and experience. Each practice constructs its patients in unique ways, offering a medical perspective with a unique truth, and a specific lens through which to gaze; each constructs a different patient body - and each has its own limitations and blind spots.

If we accept this reading of Foucault, that the medical gaze in some way creates the patient, then how can the objectifying gaze be interpreted? Returning to Foucault, "The object of discourse may equally well be a subject, without the figures of objectivity being in any way altered" $(B C \mathrm{xv})$. In short, Foucault's medical gaze simultaneously objectifies and creates the subjective patient and the patient co-produces the facts of disease with the physician. Similarly, Leder's interpretation is: "The body is not simply a thing in the world, but an intentional entity which gives rise to a world. Yet to be the latter is not to negate the former. While the body has a subjective role, it is also a body-object, a material thing" (27). Expanding this thought, Blaxter notes, “objectification does not necessarily entail loss of agency" (CVP 763). While objectification can rule out patient subjectivity, it does not necessarily do so; Foucault and others argue that the two states can co-exist. ${ }^{47}$ For the sake of this dissertation, it will suffice to assume that, as Mol and Law assert, the patient is both object and subject (87). From this foundation, we can acknowledge that:

What defines the act of medical knowledge in its concrete form is not, therefore, the encounter between doctor and patient, nor is it the confrontation of a body of knowledge and a perception; it is the systematic intersection of two series of

\footnotetext{
${ }^{47}$ Armstrong takes Foucault's complex perception of the patient as both object and subject one step further, suggesting:

But what if, following Foucault, there was no ordinary individuality, no autonomy, no discreet body, prior to the advent of the hospital and its clinical techniques...? Then, the process of corporeal objectification becomes not a destructive assault on human individuality but the very practice through which that individuality is given a literally solid foundation and manifestation (21-22).

This step, however, seems too philosophical and extreme for present practical purposes.
} 
information, each homogeneous but alien to each other-two series that embrace an infinite set of separate events, but whose intersection reveals, in its isolable dependence, the individual fact (Foucault BC 34).

This conception of medical knowledge, one that is co-produced between the patient and the physician, seems to resemble the therapeutic alliance formed in an ideal patient-centred approach. Unfortunately, patients still encounter the biomedical bias in hospital contexts.

In Discipline and Punish, Foucault introduces the idea of "docile bodies" (135); while focusing on soldiers, he also discusses naval hospital patients. The hospital is a place where "medical supervision" includes, "fiscal control over commodities, administrative control over remedies, rations, disappearances, cures, deaths, simulations" (144). Some of these controls continue to exist in hospitals today. Patients are expected to be compliant, docile, and disciplined; failure to do so may garner one a reputation as a "difficult patient" and may lead to less or begrudging attention. Because of the context in which they were raised, older patients tend to behave in an appropriately deferential manner towards physicians. Unfortunately, sometimes this means that they fail to ask questions or clearly express themselves in a medical context.

During older patients' lives, many new medical technologies have been developed to monitor patients. As nursing instructor Margarete Sandelowski observes, by the 1950s, "This [medical] technology was oriented largely to surveillance, not treatment or comfort" (136). Nurses, and other healthcare professionals, arguably became less focused on their patients, and more focused on the complexities of reading, interpreting, and communicating highly complex technical data with other professionals. Citing William Ray Arney, Sandelowski notes that "surveillance in these contexts is an intervention of social control, as opposed to purely benign 
clinical assessment" (137). Healthcare professionals have an authority to observe, to screen, to control, and medical technologies help support their power over patients.

The notion of surveillance reignites Foucault's comparison of the hospital to the prison, with philosopher Jeremy Bentham's Panopticon incorporated first through architecture, and now through technology. Foucault describes Panopticon design in detail in Discipline and Punish, citing it as a plan in which an individual might be observed from a central command post due to its semicircular, cruciform, or star-shaped plan $(200,250){ }^{48}$ "It is polyvalent in its applications; it serves to reform prisoners, but also to treat patients, to instruct schoolchildren, to confine the insane, to supervise workers, to put beggars and idlers to work" (Foucault, DP 205). In contemporary hospitals, electronic patient monitors relayed to a centralized computer console now support this kind of technological Panopticon. As with the architectural Panopticon, patients are oblivious as to whether they are being observed at any given moment. As the Panopticon acts to "induce in the inmate a state of conscious and permanent visibility that assures the automatic functioning of power," from a Foucauldian perspective, such monitoring is perceived as a way for authority to control, discipline, and normalize bodies, whether in the prison or the hospital (DP 201). The Panopticon, whether architectural or technological, exerts a complex power on those in its sightlines; "The theme of the Panopticon [is] at once surveillance and observation, security and knowledge, individualization and totalization, isolation and transparency" (Foucault, $D P$ 249). Rather than focusing on monitoring, this dissertation focuses on another type of surveillance, imaging technologies, in which the authorized medical gaze is more obvious to patients. In his study of MRIs, sociologist Amit Prasad dubs digital imaging technologies, of the

\footnotetext{
${ }^{48}$ The University College Hospital in London is also known as the Cruciform Building because of its star-shaped architecture. Designed by Alfred Waterhouse, and built in 1906, it is hard not to think of it as a Panopticon (UCL). This notion is reinforced by its location on Gower Street, opposite the Wilkins Building of the University College of London's main campus. Philosopher Jeremy Bentham's “auto-icon” resides in the Wilkins Building's South Cloister.
} 
type encountered in this dissertation, as "cyborg visuality" in which, "images have become bits of data in cyberspace that can be, and are, manipulated by human beings" $(292,310)$.

When a reductive clinical gaze, such as that supported by medical imaging, objectifies the patient, power shifts towards the physician. With their superior knowledge of disease and, in this case, images of the patient requiring professional interpretation, physicians still tend to hold the balance of power. As historian Bettyann Holtzmann Kevles comments, imaging technologies and their machines "denote[s] invisible power with mysterious ramifications" (189). Foucault's conception of power is not a stable hierarcy, however, but rather a maleable and dynamic weave that is prone to change. "Relations of power-knowedge are not static forms of distribution, they are "matrices of transformations"” (Foucault, The History of Sexuality 99). Although challenging to consider, especially as patients often feel unwell and have low energy, if the interpretation of the disease state is co-produced between the physician and the patient, the power balance can be altered.

\section{Conclusion}

This chapter has laid the foundation for the ensuing dissertation by defining the historical roots of both patient-centred and biomedical communication in Ancient Greece; exploring the role of Vesalius and other anatomists in creating an objectified patient body; examining the impact of the professionalization of doctors on their relationship with patients; and ending with an exploration of Foucualt's concepts of the medical gaze and power relations that inform the remainder of this study. The following chapter continues this archeology, with a focus on the development of medical technologies and imaging in the modern era. 


\section{Chapter 2: How Did We Get Here?}

\section{Part II: Advances in Medical and Imaging Technologies}

Man? A self-balancing, 28-jointed adapter-base biped; an electro-mechanical reduction-plant, integral with segregated stowages of special energy extracts in storage batteries, for subsequent actuation of thousands of hydraulic and pneumatic pumps, with motors attached; 62,000 miles of capillaries; millions of warning signal, railroad and conveyor systems; crushers and cranes (of which the arms are magnificent 23 -jointed affairs with self-surfacing and lubricating systems, and a universally distributed telephone system needing no service for 70 years if well managed); the whole, extraordinarily complex mechanism guided with exquisite precision from a turret in which are located telescopic and microscopic self-registering and recording range finders, a spectroscope, $e t$ cetera, the turret control being closely allied with an air conditioning intake-andexhaust, and a main fuel intake.

R. Buckminster Fuller, “The Phantom Captain,” 18 While the previous chapter explored the origins of the patient-centred and biomedical models, and traced the historical relationships between patients and practitioners, this chapter focusses on more recent trends in medical and imaging technologies. According to Wootton, “....medicine, at least since Hippocrates, has always been a technology, a set of techniques used to act on the material world, in this case the physical condition of the patient's body" (8). Offering a somewhat narrower definition, Rose defines technology as, “...hybrid assemblages of knowledges, instruments, persons, systems of judgement, buildings and spaces, underpinned at the programmatic level by certain presuppositions and assumptions about human beings" (16). 
Using a combination of McLuhanesque and Foucaldian langauge, nursing instructor Pascal Lehoux simply defines contemporary medical technology as “.... an extension of one's range of potential action, gaze, knowledge, and power" (59). While medical technology might be considered as the knowledge to treat the patient's body, the types of medical technologies addressed in this dissertation are object technologies, in this case, imaging technologies (both the scanners and the images themselves), more closely aligned with Rose and Lehoux's definitions. As scientific discoveries progressed through the nineteenth and twentieth centuries, medical advances altered our perceptions of the human body. Patients benefited from public health campaigns, including those for vaccines, as well as progressive notions of hygiene, such as Joseph Lister's concept of antiseptic surgery. ${ }^{49}$ Sir Francis Galton's theory of selective breeding for healthier humans was popularized through the eugenics movement in the early $1900 \mathrm{~s}^{50}$ (Bogdan, "Social Construction of Freaks" 31, 34). Between eugenics, and the institutionalization and sometimes forced sterilization of individuals deemed substandard, the

\footnotetext{
${ }^{49}$ See Chapter 1 footnotes 30 and 32 for further details.

${ }^{50}$ In the post-World War II era, society sometimes conveniently forgets that eugenics were not a purely Nazi endeavour instituted for "racial cleansing," as well as against those with mental, physical, or psycho-emotional disabilities. For instance, an article in The American Journal of Insanity published in 1882 advocates, "...stamp[ing] out insanity by castrating all the insane men and spaying all the insane women" to negate the possibility that they might pass on substandard genes (Goodell 295). The First International Congress of Eugenics was held in London in 1912, but the movement shifted to the United States with the Second and Third International Congresses held in New York in 1921 and 1932 respectively (IEC). P.T. Barnum held the first National Baby Show at his American Museum in New York in 1855; as well as considering diet and hygiene, judges also assessed whether contestants were "genuine original American stock" (American Social History Project). The first Scientific Better Baby contest was held in 1908 at the Louisiana State Fair. By the 1920 Kansas Free Fair, the notion of selective human breeding had extended to the Fitter Families for Future Firesides contests, which fell under the auspices of the American Eugenics Society. Eugenics exhibits were common at state fairs and community events throughout the 1920s and 1930s. The popular movement readily slipped into state controlled reproduction and immigration policies, as well as medically endorsed passive euthanasia of the unfit as exemplified in films like The Black Stork (Selden 207, 210$211,215,221,205)$.

The eugenics movement was also active in other countries, including Canada. Both Alberta (1928-1972) and British Columbia (1933-1973) instituted policies of forced sterilization on those deemed genetically unfit. Sterilization was undertaken on 2,822 individuals found "mentally defective" or "psychotic" by The Alberta Eugenics Board (Robertson). In 1996, Leilani Muir won her case against the Alberta Government for wrongful sterilization under the province's Sexual Sterilization Act. The SSHRC-funded Living Archives on Eugenics in Western Canada project was established in 2010 to investigate this under-researched era in Canadian history, and give voice to those still affected by the Act. Findings indicate that individuals who were poor, single mothers, East European, First Nations, or Métis were over-represented in the population that underwent forced sterilization (CURA).
} 
number of human anomalies, "freaks," or "living curiosities" as showman P.T. Barnum called them, began to drop (Bogdan, Freak Show 34; Hartzman 4). Depending on the ways in which information derived from The Human Genome Project, completed in 2003, is used, ${ }^{51}$ arguably advanced genetics could be seen as a more sophisticated continuation of Galton's work towards perfected human beings (HGP). Throughout the nineteenth and twentieth centuries, medical instruments that both extended the physician's gaze, and distanced the physician from his (or, increasingly, her) patient, were developed. By the 1970s, advanced medical imaging technologies, supporting an internal clinical gaze, had become a common diagnostic tool in Western medicine. Perhaps unintentionally, imaging technologies fragment the patient and support a reductive medical gaze by creating an informational body, an objectified conglomeration of digitized data removed from the human subject.

\section{The In(ter)vention of Medical Instruments}

When you no longer know what headache, heartache, or stomachache means without cistern punctures, electrocardiograms and six $x$-ray plates, you are slipping.

Dr. Martin H. Fischer (1879-1962)

Prior to the development of medical technologies, doctors relied primarily on the patient's narrative of symptoms, and their own observations of signs of illness; occasionally, a manual examination might be performed, although social decorum frowned on such intimacy, especially

\footnotetext{
${ }^{51}$ In June 2013, the American Supreme Court ruled against Myriad Genetics, a company arguing for a patent on the BRCA1 and BRCA2 genes used for breast cancer screening. The court concluded that, "A naturally occurring DNA segment is a product of nature and not patent eligible merely because it has been isolated" (Liptak A1). While this ruling makes screening more available for women at risk, the fact that a court decision was required to retain control of our human genetic information may be indicative of future trends. While genetic information may benefit some patients, the HGP can also derive corporate profit, depending on the ways the information is used, adapted, and copied.
} 
in the upper classes of the modern era. As Reiser asserts, however, doctors were ambivalent about the reliability of patients' narratives, as such stories did not provide an objective account of disease; "Doctors complained that when questioned patients were often too talkative or introduced irrelevant data, or that when anxious they might exaggerate symptoms, or that when shy or embarrassed about a condition they might withhold evidence" ("Technology and the eclipse of individual in medicine" 11). Physicians' own observations, and perhaps a limited physical examination (checking the pulse, skin texture, and palpating for tenderness) were perceived as more reliable, objective measures of the patient's disease state (Porter, CHM 83-84; Reiser, MRT 1-4). Previous generations of medical researchers, like Bichat, who had noted signs of disease in living patients and then confirmed their findings through autopsies, had provided reliable causal links between signs and taxonomies of disease, as well as the anatomical changes caused by specific disease states (Reiser, MRT 114). This accumulated knowledge, with the addition of advances in medical technologies, allowed practising physicians to diagnose their patients with decreasing reliance on patients' perceptions.

The move towards increased medical technology, in terms of technique, began with Leopold Auenbrugger's (1722-1809) 1761 monograph on percussion, Inventum nouvum. Criticizing patient accounts as "inconstant and untrustworthy," Auenbrugger developed percussion, a method of tapping on the surface of the body to objectively determine abnormalities in underlying structures (Ackernecht 136; Porter, GBM 308; Reiser, MRT 20). Although Auenbrugger's use of percussion proved too complex and intimate for most practitioners, it initiated the idea of transforming auditory into visual information, a notion that continued to develop into the stethoscope and ultrasound devices, and facilitated a non-surgical internal examination on a living patient. 
Concerned with patients' "prejudice and ignorance," René-Théophile-Hyancinthe Laennec (1781-1826), a pupil of Bichat, developed "mediate auscultation" in 1819, although his invention of the stethoscope was essentially accidental (Laennec in Reiser, MRT 31, 23;

Ackernecht 151-152; Porter, CHM 153). While a doctor could gain helpful auditory information by placing his ear on the patient's chest, doing so breached both social and professional boundaries. One of Laennec's patients, a rather large-bosomed woman, had a heart condition. To facilitate the necesary physical examinaton, while maintaining decorum, Laennec rolled a paper cylinder; the improvised instrument allowed him to hear her heart clearly while retaining an appropriate physical distance. Laennec developed the wooden monocular stethoscope in 1816, and in 1819, he published the 928-page Traite de l'Auscultation mediate (Treatise on Mediate Auscultation) (Porter, CHM 153, GBM 309; Reiser, MRT 25). Resier asserts that this volume, "reformulated the relationship between doctors and patients through the use of an instument that took the mantle of illness out of the hands of patients and placed it in the doctor's orbit" (TM 7). Although the stethoscope met with initial resistance from some patients and physicans, its use was standard practice by the 1850s (Porter, CHM 83-84; Reiser, MRT 25, 38).

Listening for bodily abnormalities allowed doctors to visualize anatomical changes, and thereby improve their diagnositic skills (Porter, GBM 308; Reiser, MRT 29-30). For example, a healthy patient's breathing and coughing sound different from a tubercular patient's. A 1911 article in the Journal of the American Medical Association asserts that when diagnosing tuberculosis, auscultation with a stethoscope is more sensitive than percussion or "Röntgen rays" (the original name for X-rays), because "fine râles," or crackles, can only be discerned with a stethoscope (Smith 245). As one physician declared in a London medical journal in 1835, "We anatomise by auscultation (if I may say so), while the patient is yet alive" (Latham in Reiser, 
MRT 30). More recently, Leder linked the development of medical technologies to the profession's obsession with cadavers: "Such technologies as the stethoscope, the blood test, the X-ray, allow a kind of dissection of the living body, analyzing it into its component parts, exposing what life ordinarily conceals" (22). The notion of anatomizing the living patient, of using technology to derive objective information, rather than relying on the patient narrative, underscores the immediate tendency for even simple technologies to objectify the patient. As Reiser notes, "With the transferring of doctors' attention from the words spoken by patients to the sounds produced by their organs, the beginnings of modern therapeutic distancing arrived" (TM 12).

Other than the stethoscope, most nineteenth-century diagnostic developments extended the doctor's visual sense. Early modern doctors had made diagnostic use of all five senses; “...they would feel the pulse, sniff for gangrene, taste urine, listen for breathing irregularities and observe skin and eye colour" (Porter, GBM 256). By the turn of the century, in 1899, relentless medical invention caused one physician to complain, "We have lost almost completely the intellectual use of the sense of smell, and are gradually relegating hearing to a lower intellectual plane than sight" (Benedict 684). Specialized instruments, like ophthalmoscopes (1851) and laryngoscopes (1855), allowed doctors to peer into patients' bodies through natural orifices (Porter, GBM 385, 605). Springing from the success of these instruments, more intrusive tools were developed to examine the bladder, rectum, and stomach; although the vaginal speculum was developed in the 1860s, its use was viewed as "immoral and offensive" (Reiser, MRT 55). The Edison lamp, invented in 1881, provided stronger illumination, further emphasizing the use of the visual sense for examinations (Reiser, MRT 55-56). As diseases like typhus (smelling of 
mice), the plague (smelling of apples), and yellow fever (smelling of plucked feathers) became less common, physicians' use of diagnostic smells became less relevant (Ackerman 54). ${ }^{52}$

In addition to instruments that extended physicians' sight, devices were also developed to record data based on a patient's organ function. These included the spirometer for respiration (1846), the sphygmomanometer for blood pressure (1896), and the electrocardiogram (ECG or EKG) for heart activity (1903). Such technologies, "had the capacity themselves to sense evidence and, most critically, to transform it into the objective forms of pictorial, graphic, or numerical data" (Reiser, TEI 11). Improved microscopes (1830) revealed cells and bacteria, and continual investigation furthered the understanding of the body's biochemical composition (Ackernecht 158; Porter, GBM 223-226, 344, 582; Reiser, MRT 44, 121).

All of these advances increased the quantity of objective information available to doctors, further reducing their reliance on patient narratives. New medical technologies encouraged doctors to isolate affected areas of the body for examination, rather than considering the patient holistically; Reiser asserts that the trend for physicians to look at parts, rather than the whole patient, in their efforts to identify disease, began with the work of the early anatomists (TM 186). During the 1800 s, some physicians expressed concern at the loss of manual skills for physical examinations, as well as the lingering association of medical instruments to manual labour, such as surgery. A few physicians also expressed concern about the decreasing dialogue with patients (Reiser, MRT 36; Sandelewski 92).

Increasing reliance on technologies led doctors to believe that medical instruments were more reliable diagnostic tools than subjective information gathered from patients (Kevles 81;

\footnotetext{
${ }^{52}$ Researchers are now re-examining the efficacy of diagnostic smells by using dogs to detect cancers. See Cornu, et al.; Horvath, Andersson, and Paulsson; McCulloch et al.; Moser and McCulloch; Sonoda et al.; Willis et.al. See also the Pine Street Foundation and InSitu Foundation websites, as well as the British documentary, Can Dogs Smell Cancer? (2005).
} 
Reiser, MRT 170; Reiser, TEI 12; Sandelewski 92-93). “The doctor who depended chiefly on technology in diagnosing and following the course of illness could think of himself as using the same rigorous methods as did the scientist who pursued truth in his laboratory" (Reiser, $M R T$ 161). Similarly, Porter suggests that, “...modern medicine promotes its claim to be scientific, and hence to be as attentive to the objective laws of disease as, say, physics is to particles" ( $C H M$ 85). Lock and Nguyen make a similar observation (20). Physicians want definitive answers to help treat their patients, and some believe that "the laboratory will supply a clear-cut diagnosis, certainty in place of doubt" (Garrod 63). Physician C. Ward Crampton declared in a 1930 article on "Synthetic Diagnosis" in Medical Clinics of North America, "Let me test the patient, I don't need any history," a notion reminiscent of Medieval physicians requiring only a urine sample for diagnosis (Crampton in Reiser, MRT 170; Wootton 63).

Wootton equates the adoption of the thermometer in the mid-1800s with the death of Hippocratic medicine, as it denied the need for the physician's hand to determine subtleties in the type of heat a fever produced (54). Increasing trust was placed in numerical or machineproduced data. The thermometer, and similar technologies, "had the effect of reducing patients to simplistic and reductionist 'readings,' transforming subjective judgement and affective relations to new metrics of surveillance and monitoring" (Brandt and Churchill in Sandelewski xiii-xiv). Subjectively collected data, like the patient's pulse or respiratory rate, could be recorded numerically, because, "Medical facts recorded in numbers or graphs gave an appearance of exactness and finality" (Reiser, MRT 162). As visual communication sociologist Luc Pauwels contends, however, this type of information is not based on "physical objects or phenomena, but 'data' that are constructed by observing aspects of the physical world. The relationships among the data and their representation are much more abstract/arbitrary and conventional...” (150). 
Objective data, information removed from the subjective presence of the patient, could be shared and discussed among medical colleagues, providing greater assurance, to both physicians and patients, of a correct diagnosis (Reiser, TEI 11). The production and collection of objective data seemingly required little training or judgement, allowing doctors to delegate certain technical tasks to nurses or technicians (Reiser, $M R T$ 117; Sandelewski 68, 83). There were, however, voices of concern that laypeople, or inadequately trained technicians (or doctors), might provide unreliable data, owing to their lack of proficiency with a given instrument (Reiser, MRT 94).

In the early twentieth century, public pressure encouraged doctors to use all technologies at their disposal. Patients perceived medical technologies as more accurate, objective, and reliable than the physician's judgement alone. Some patients demanded unnecessary X-rays as evidence of surgeries performed; doctors were sometimes sued for malpractice for not using all available tests. In short, patients colluded with doctors to subject themselves to unnecessary tests and X-rays, sold on the infallibility of new medical technologies through the power of the press (Porter, GBM 671; Reiser, MRT 163-164). As a result, doctors became less confident in their own clinical judgement, reducing their diagnostic abilities; essentially, they became managers and interpreters of information between patients and specialists or technicians (Reiser, $M R T$ 173). Reiser comments that, "...the patient was less a person and more an object of study, and the doctor more a biologist than a physician" (MRT 166). As a consequence, professional techniques developed in the nineteenth century — observation, palpation, auscultation - fell out of favour by the 1930s, and were less frequently taught or practised (although they have since been reintegrated). Once X-ray technology and biochemical tests had permeated practice, the patient exam and earlier technologies like the stethoscope, were viewed by many physicians as irrelevant, imprecise, and time-consuming (Joyce 443; Reiser, MRT 169). 
Until the early 1900s, people visited doctors only when they themselves recognized symptoms of illness; patients were responsible for alerting doctors to disease states. That changed in May 1923, when Dr. Haven Emerson, Chairman of the American Committee of Council on Health and Public Instruction, published an article in the Journal of the American Medical Association entitled, "Periodic medical examinations of apparently healthy persons," establishing the notion of routine check-ups for asymptomatic people as a mode of preventative medicine. An ad campaign, building on the public's burgeoning desire for automobiles, was launched, encouraging people to book preventative health check-ups. Campaign posters, echoing the Cartesian model, quoted text from Emerson's article: "Your body is a wonderful machine. You own it and operate it. You can't buy new lungs and a heart when your own are worn out. Let a doctor overhaul you once a year" (Emerson in Reiser TM 143). Various tests and screening procedures have been added to, or lost from, the routine physical over the ensuing decades. Emerson's article heralded the medicalization of healthy subjects, and shifted responsibility for identifying disease to the physician. Now a patient who felt healthy, might be deemed ill through routine testing. The positive result of this shift was the possibility of earlier disease identification, treatment, and better outcomes (although few effective treatment options existed at the beginning of the twentieth century). The negative result for patients was that it stripped them of a level of power regarding their own bodies; people became reliant on doctors to inform them when they were unwell, and test-based, clinically intepreted judgements of their physical state might take precendence over their subjective bodily experience.

In American hospitals, the increase in routine, but unnecessary, testing led to what was perceived by some as an "over dependence on technology" that sometimes resulted in substandard medical treatment (Reiser, MRT 158). A 1912 article written by a visiting French 
physician suggested that in American hospitals, “...the diagnosis and treatment of a given patient depended more on the results of these various tests than on the symptoms present in the case" (Austin 801). By the 1930s, hospital admission required the testing of blood and urine samples, as well as X-rays (Sandelewski 82). Studies examining the hospital use of medical technologies also began in the 1930s, noting a staggering increase in testing over time, due in part to the packaging of tests to improve the economics of scale for automated laboratories (Reiser, $M R T$ 159). ${ }^{53}$ The number of tests performed, the density of data accumulated, was overwhelming to physicians, creating a "glut of medical data," with the unfortunate side effect that pertinent abnormal findings might be overlooked (Reiser, MRT 195). In the twentieth century, "The hospital was no longer primarily denounced... as a gateway to death but as a soulless, anonymous, wasteful and inefficient medical factory, performing medicine as medicine demanded it, not as the patient needed it" (Porter, $B G$ 151).

Patient diagnostics were no longer perceived as the intellectual puzzle ${ }^{54}$ it had been for earlier generations of physicians, but rather as cases to be managed through tests and specialists. Increasingly, evidence was collected through the examination of lab results and images, rather than the physical examination of, and discussion with, patients. In a 1976 editorial in the Journal of the American Medical Association, Dr. George Engel observed common errors in physical examinations. He noted that medical students had only one or two supervised patient encounters during their training; with such limited exposure to patients, it is hardly surprising that

\footnotetext{
${ }^{53}$ For instance, a 100\% increase in the number of tests performed during a single hospital stay between 1938 and 1958 was documented at a Michigan hospital; a ten-year study started in the mid-1950s, showed that the number of routine tests doubled every five years; by the 1970s, this rate was increasing even faster, so that the two billion tests performed on American patients in 1971 had increased to three billion tests by 1974. While these are American findings, Reiser notes similar increases in both the U.K. and Canada (MRT 159).

${ }^{54}$ The notion of the medical case as an intellectual puzzle is exemplified in the work of Dr. Joseph Bell. Dr. Bell's use of detailed observation and logical deduction to formulate correct medical diagnoses inspired his student, Arthur Conan Doyle, to create the famous amateur detective Sherlock Holmes, who uses these same powers to solve nonmedical mysteries (Chalmers).
} 
physicians' observation, physical-examination, and history-gathering skills were inadequate. Engel blames these shortcomings on the profession's over-reliance on technology, declaring: Physicians of today are ill-equipped to respond to the problems brought by their patients other than by suggesting more diagnostic tests. A negative laboratory workup is considered tantamount to no illness, while deviation from the statistical mode may be accepted as evidence of disease even among those who are in every respect well. The personal relationship with patients is attenuated and distorted, for physicians too heavily depend on data reported by sources extraneous to both themselves and the patient. Time is disproportionately devoted to waiting for and evaluating laboratory data, rather than to interacting with the patient. For the latter, the doctor is replaced by the awesome power of the laboratory, and in the process both patient and physician are diminished. (862)

Supporting Engel's observation, Reiser comments that the increasing reliance on technology came at the expense of a decreasing physician confidence in clinical judgement, and an increasing disregard of the patient's illness experience (170). In short, some physicians seemed more concerned about interpreting objective data than they were about their patients' subjective wellbeing.

Increasing confidence in diagnostic medical technologies actually increased the number of medical errors. A seven-year study at the Veterans Administration Hospital in Washington, in which 1,106 deceased patients were autopsied, found that $6 \%$ had been misdiagnosed, and that the rate of misdiagnosis increased from $1.5 \%$ in 1947 to $9 \%$ by 1953 (Gruver and Freis 108-109). The most frequent fatal misdiagnosis was accute infection (treatable by that time with antibiotics), sometimes with an atypical presentation, followed by brain tumours, intestinal 
ulcers, and cardiovascular disease (Gruver and Freis 110-111). The primary cause of misdiagnosis, affecting $45 \%$ of these cases, was the lack of a patient history, for various reasons

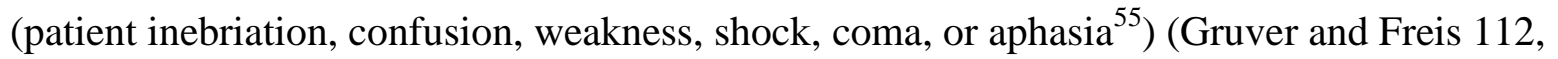
118). Other reasons for misdiagnosis included errors in clinical judgement caused by failing to account for all of the patient's symptoms and observable signs (i.e., ignorning the patient's verbal [narrative] and/or non-verbal [body language] communication) (Gruver and Freis 119, 113). In some cases, lab or imaging results were ignored. In others, the imaging results were normal, diverting the physician from a planned course of action by incorrectly ruling out a suspected cause; although subsequent examination found no error either technically or interpretively, the X-rays simply failed to reveal necessary information for the correct diagnosis (Gruver and Freis 113, 116). In one case, although his X-ray findings were interpreted as normal, an interpretation confirmed on secondary examination, a man died from a skull fracture and accute brain injury (Gruver and Freis 116). The study's authors, Robert Gruver and Edward Freis, suggest that medical technologies create a "false sense of security engendered by misleading negative laboratory reports, particularly x-ray films which did not disclose the lesion" (118). This 1957 study supported the findings of a 1952 study undertaken by Willy Munck in Denmark.

Delegating responsibility for diagnostic data gathering and/or interpretation potentially creates an ethical problem too. Reiser suggests that the diagnostic ideal is a collaborative effort between the doctor, possibly an assistant he or she directly oversees, the technician or specialist, and the patient, in which final decisions are jointly determined. When a doctor sends a specimen to the lab, however, there is no communication between the lab and the patient. The lab report is

\footnotetext{
${ }^{55}$ Aphasia is derived from the Greek, aphatos, meaning speechless (Canadian Oxford Dictionary). The condition is caused by brain injury or damage, resulting in an inability to understand or communicate language, either verbally or in writing (Mayo Clinic).
} 
returned to the physician, who may or may not have the expertise to evaluate potential lab errors. This lack of verification of diagnostic results is viewed by Reiser as a dereliction of professional responsibility (Reiser, MRT 171-2; Reiser TEI 13-14). It is also common Canadian practice. For instance, a patient may be sent for imaging, undertaken by a technician, and then interpreted by a radiologist who has no direct communication with the patient. The radiologist returns a report to the physician, who then discusses the results with the patient, but generalist physicians rarely view the images, and may not have the expertise to evaluate potential technical or interpretive errors (Potchen 424). ${ }^{56}$

While there is no doubt that advances in medical technologies have extended many lives, they also raise an inconvenient problem: interpretation. Technologically derived evidence is perceived by both patients and most doctors as objective data, and therefore accurate and correct. All data must, however, be interpreted, and this interpretative step is subjective. For instance, if, on a routine blood test, the values fall "within normal ranges," the patient may be declared healthy, even if she or he feels ill. Is the patient malingering? Is the illness psychosomatic? Or is the technology simply too coarse to detect subtle changes of which the patient is subjectively aware? Perhaps the patient's disease state is not yet acute enough for technological detection. For instance, Dr. James Wilson, and others, argue for a condition called "adrenal fatigue" not clinically recognized. If a patient with adrenal fatigue continues to decline into adrenal

\footnotetext{
${ }^{56}$ This describes my own recent experience. Technicians for various imaging types forwarded their technologically derived images to a radiologist, someone with whom I had no direct communication. The radiologists' reports were forwarded to my family practitioner (FP), but she did not examine the actual images. The information I received were the radiologists' interpretations, their written reports, quickly read during the appointment and filtered through my FP. These images have only been viewed and interpreted once, by a radiologist with limited written knowledge of the patient. As will become apparent in this dissertation, my experience was not unique, and goes against recommendations for at least two professionals to view and interpret images to decrease the possibility of misdiagnosis.

${ }^{57}$ The adrenal glands produce hormones that help regulate bodily functions, including heart rate, immune response, and energy allocation, in direct relation to physical and psycho-emotional stressors. According to Dr. Wilson, adrenal fatigue occurs when an individual is exposed to extreme and/or multiple stressors over a prolonged period, and the adrenal glands can no longer meet the body's hormonal demands (Wilson; Adrenal Fatigue).
} 
insufficiency, or Addison's disease, then she or he has a clinically recognized disease and is treated. A patient might have a blood test showing that her or his adrenal function is "within normal ranges," even when functioning at the low end of normal, or even when functioning has decreased from that individual's normal range, causing the patient to feel unwell. Clinically, the patient would still be considered healthy, until or unless her or his values continued to decrease into the range recognized as adrenal insufficiency (Wilson; Adrenal Fatigue). As medical anthropologists Margaret Lock and Vihn-Kim Nguyen observe, "it is commonly assumed in the medical sciences that the human body is readily standardizable by means of systematic assessments" (20). This sort of standardization, or normalization, might also be referred to in Foucauldian terms as a disciplining of patient bodies. Raw objective data requires a human interpreter, someone to decide whether a health problem exists, and if so, how it should be treated. The interpretation of data destabilizes the notion that technologically derived data is purely objective (Herrick 520; Reiser, MRT 182-3).

The accuracy of technologically supported medical diagnostics was further destabilized by several studies undertaken from the 1950s to the 2000s providing evidence of observer error, subjective misinterpretation, mechanical error, lack of lab training or regulation, incompetence, or poor inter-professional communication (See, for example: Anderson, Hill, and Key; Butterworth and Reppert; Fletcher; Goswami et al.; Gruver and Freis; Koran; Plebani; Roosen et al.; or Stradling and Johnston) ${ }^{58}$ Reiser observes that, "Precision in medical diagnosis seems to depend on three characteristics: the intrinsic accuracy of the measurement or test, the constancy of the phenomena being measured, the ability of the observer to interpret and record the

\footnotetext{
${ }^{58}$ According to investigative journalist Maryn McKenna, the number of autopsies performed in the U.S. declined from about $20 \%$ of deaths in the 1970 s, to $8.5 \%$ of deaths by 2007 . While this decrease may be partly due to costs, McKenna also contends that, in the litigious atmosphere of the American healthcare system, the capacity for autopsies to reveal medical errors is not seen as desirable by some physicians or hospitals. McKenna's article examines the mixed results of using CT and MRI in virtual autopsies, or "virtopsies."
} 
phenomena" (194). Increased automation, oversight, and regulation of medical laboratories has had limited impact on error reduction, yet has had the effect of making physicians even more trusting of lab results, and even less likely to argue with them, even when the results make little sense for an individual patient, and even though subjective data interpretation is involved (Berlin 1174; Resier, MRT 189).

The explosion of available medical technologies in recent decades is a double-edged sword for patients and physicians, offering previously unavailable solutions, but new problems as well. Lehoux admits that "technology is dehumanizing," not because it is technology, but because of the ways it can be used (xvi). In a contemporary hospital setting, where staff is overburdened and efficiency demanded, monitors support the simultaneous observation of several patients from a central console. There is, however, a risk that patients can become nothing more than objective data on a screen. Nursing educator Rozzano Locsin suggests, "Technique marginalizes the incorporation of subjective and non-technical phenomenon (e.g., human experience) by either negating its importance or framing it within rational and organized order" (29). Technologies require and allow medical personnel to distance themselves from sick and dying strangers. Medical technologies tend to create vast quantities of "bodiless information," flattened datasets of minutiae that are only tangentially attached to the living patient's body through barcodes (Hayles 22; Rose 14).

The tension created by technologies in the medical context is perhaps most glaring in end-of-life care and the determination of death. ${ }^{59}$ Not long ago, death seemed obvious, something even an untrained individual could ascertain (although perhaps with dubious accuracy); when people stop breathing, and their hearts stop beating, they are dead. In the last

\footnotetext{
${ }^{59}$ For a longer discussion of the medicalization of death, see my book chapter, "Life Extension, Immortality, and the Patient Voice," in The Power of Death: Perceptions of Death in the Western World (New York and Oxford: Berghahn Books, 2014).
} 
few decades, as medical technologies have advanced, the determination of death has become more difficult to ascertain. In an attempt to clarify the death state, in 1968, an Ad Hoc Committee of the Harvard Medical School published "A Definition of Irreversible Coma" in the Journal of the American Medical Association. By 1968, medical technologies existed that could "restore 'life' as judged by the ancient standards of persistent respiration and continuing heart beat" (Beecher et al. 87). In this landmark article, the committee concluded that medically, death could be defined by irreversible coma. Irreversible coma, or brain death, is the point at which brain activity can no longer be detected, a point that must be determined using electroencephalograhic (EEG) equipment to ascertain the presence, or lack, of a brain's electrical activity. If brain death was determined, the patient could be considered medically and legally dead, and so he or she might be removed from mechanical life support.

According to Google Scholar, the "Definition of Irreversible Coma" article has been cited in 89 other publications to date, and the article's abstract has been cited in 315 publications, evidence of the ongoing discussion around the contemporary medico-legal definition of death. Additionally, these articles include: debates about whether the whole brain must cease to function, or only certain critical parts of the brain, to declare death (See, for example: Bernat, Culver, and Gert; or Bernat, 1992, 1998); ${ }^{60}$ the relationship between brain and body, and whether brain death is the most suitable criterion by which to determine death (See, for example: Parisi et al.; Shewmon, 2001; or Verheijde, Rady, and McGregor); the detection of heart activity, after brain activity has ceased (See, for example: Ganes and Lundar); and most recently, the possibilities that simple communication with people in an irreversible coma can be achieved

\footnotetext{
${ }^{60}$ While most practitioners agree that whole brain death is necessary to declare death, a small faction insists that once higher order brain functions cease, death may be declared. The brain stem, which is responsible for lower order bodily functions, however, remains active in a persistent vegetative state (PVS), from which people can regain consciousness.
} 
using functional magnetic resonance imaging (fMRI), and that brain death may be reversible (See, for example: Matsuda et al.; Naci et al.; Owen et al.; or Shewmon, 2009. See also other publications of the Owen Lab at the University of Western Ontario).

The accurate determination of death has become increasingly important for two primary reasons, both cited in the original 1968 article: the limited availability of hospital beds makes it desirable to free space when patients have no hope of recovery; and once death is legally determined, a patient's organs potentially become available for transplantation. Brain injuries, of the type that might cause an irreversible coma, often leave the rest of the body healthy, intact, and ideal for organ "harvesting." Through research, as well as clinical trial and error, transplant surgeons have determined that living donor organs produce better results than cadaveric donor organs; living organs are usually healthier, begin functioning more quickly once transplanted, and survive longer after they are transplanted (Kidney Foundation). A brain-dead patient on life support is an ideal candidate for organ "harvesting." In a very rationalized way, as Waldby comments, "donor cadavers can be transformed into resources of vitality for the living" (147). (Un)fortunately, cases do exist of patients waking from comas that, correctly or incorrectly, were deemed irreversible (three such cases are discussed in Matsuda et al.). Such exceptions cause physicians, and the general public, to consider the possibility that, based on current medical technologies and medico-legal definitions, some patients who might have recovered, have inadvertently been unplugged so that their organs could be harvested. While it is likely that someone regaining consciousness from such a brain-damaged state would have limited function and a questionable quality of life, should physicians and lawyers be making these types of life and death determinations? 
Once a technology exists, there is a tendency to use it, whether or not it is advantageous or ethical in a given situation, or for a given patient. As a final caution, Porter alludes to: ...the high-tech 'can do, will do' approach apparently embraced by scientific medicine at the cutting edge-medicine led by an elite that sometimes seems primarily interested in extending its technical prowess, with scant regard for ends and values, or even the individual sufferer. Where patients are seen as 'problems' and reduced to biopsies and lab tests... (CHM 7)

In some cases, available technologies may seem to give physicians an almost godlike power over disease, over life and death, even over their patients—-but physicians are not gods, they are merely humans like the rest of us, and just as capable of errors in judgement. More critical judgement, and improved communication, about the ways in which medical technologies are used, might help create a better balance between patient-centred and biomedical practices.

\section{The Uncanny Magic of Medical Imaging Technologies}

No previous diagnostic discovery had stirred quite so much public interest and involvement as the X-ray. It obliterated one distinction between the outer and inner spaces of the body-both were now susceptible to visual examination.

Dr. Joel Stanley Reiser (MRT 62)

In 1895, physicist Wilhelm Röntgen discovered a use for X-rays, or Röntgen rays, a form of electromagnetic radiation, while experimenting with a Crookes vacuum tube. ${ }^{61}$ The first X-ray

\footnotetext{
${ }^{61}$ Among other things, researcher William Crookes (1832-1919) experimented with vacuum tubes. Passing electrodes through the base of a vacuum sealed glass tube, similar to an incandescent light bulb, allows electricity to be contained and controlled (Jenkins, J.). Prior to the invention of transistors and microchips, early electronic equipment required tubes.
} 
image was that of Anna Röntgen's hand, complete with her wedding ring (Cartwright 111). ${ }^{62}$ To successfully capture the image, the X-ray exposure was between fifteen minutes and half an hour (Dam 414; Kevles 20). Upon seeing the image, Frau Röntgen reportedly exclaimed, "I have seen my death!" alluding to the uncanny sense many non-clinicians felt at the advent of X-rays (Wellcome Trust; Kevles 38; St Louis Post Dispatch in Reiser, MRT 60). Although early images rendered considerably less detail than contemporary anatomical drawings, ${ }^{63}$ X-rays were adopted for medical imaging within weeks of Röntgen's publication, and several journal articles appeared in early 1896 (Reiser, MRT 60). In 1896, for instance, Professor Henry Cattell wrote, "It is even now questionable whether a surgeon would be morally justified in performing a certain class of operations without first having seen pictured by these rays the field of his work - a map, as it were, of the unknown country he is to explore" (in Reiser, TM 23). At the end of World War I (1918), X-ray machines were considered specialized equipment in some hospitals and medical offices; by World War II (1939-1945), their use had been accepted as standard practice in hospitals, as well as medical and recruiting offices (Kevles 74, 141).

Perceived as an extension of photography, X-rays were readily accepted by both physicians and the public. Although photography was still an unwieldy infant technology, as early as the 1850 s, doctors debated the possibility of incorporating photographs into the objective medical record, for instance, as evidence of treatment over time (Reiser, MRT 56-62; van Dijck 89). McLure's Magazine journalist H.J.W. Dam declared in April 1896:

That a new photography has suddenly arisen which can photograph the bones, and, before long, the organs of the human body; that a light has been found which

\footnotetext{
${ }^{62}$ The original X-ray is archived at the Francis A. Countway Library of Medicine, affiliated with the Harvard Medical School in Boston (Reiser 59). A photographic print of the image is also housed in London's Wellcome Trust Collection and available for online viewing.

${ }^{63}$ Röntgen's discovery occurred just thirty-seven years after the publication of the first edition of the very detailed engravings of Henry Gray's Anatomy: Descriptive and Surgical (1858).
} 
can penetrate, so as to make a photographic record, through everything from a purse or a pocket to the walls of a room or a house, is news which cannot fail to startle everybody. That the eye of the physician or surgeon, long baffled by the skin, and vainly seeking to penetrate the unfortunate darkness of the human body, is now to be supplemented by a camera, making all the parts of the human body as visible, in a way, as the exterior, appears certainly to be a greater blessing to humanity than even the Listerian antiseptic system of surgery; and its benefits must inevitably be greater than those conferred by Lister, great as the latter have been (408).

The press stirred public interest in X-ray technology, spawning lectures, displays, public X-ray slot machines and, arguably, creating unrealistic expectations (Howell in Kevles 25). Popular interest led to X-rays becoming a curiosity, a form of entertainment; people subjected everyday items, like wallets and keys, to X-rays for keepsake images (Dam 412; Reiser, MRT 60). An 1898 New York Times article indicates the popularity of women sitting for purely decorative Xray portraits, ${ }^{64}$ especially "intimate photographs" of their hands donning jewelry (Cartwright 115; van Dijck 89). Dr. William J. Morton, whose work appeared in both academic journals and the popular press, ${ }^{65}$ assembled a gallery of X-ray portraits from human appendages with embedded foreign objects, to hands and feet fastened inside gloves and boots. Dr. Morton, who was also responsible for the "frivolous" full-sized, single-exposure X-ray portrait of a woman, declared that there was no discomfort in having an X-ray photograph taken, "Nor is there any

\footnotetext{
${ }^{64}$ An issue of Punch magazine published shortly after Röntgen's discovery (January 25, 1896), featured a poem entitled "The New Photograph," in which the author pleads with the inventor, "We only crave to contemplate / Each other's usual full-dress photo; / Your worse than 'altogether' state / Of portraiture we bar in toto!' (cited in Reiser, MRT 61). Although there was general public acceptance, there may also have been some disbelief or discomfort with the idea of X-rays, leading to a certain critical or satirical reflection. Privacy concerns were also raised, due to fears that X-rays could be used to peer through walls and into people's homes (Reiser, MRT 61).

${ }^{65}$ In 1896, Dr. Morton co-authored a technical book entitled The X Ray or Photography of the Invisible and its Value in Surgery, which included a series of early photographic X-rays plates.
} 
danger," when the equipment was properly used. Many early practitioners set the X-ray tube close to the patient's skin and gave long exposures, sometimes causing radiation burns; Dr. Morton used a conservative five-minute exposure at three feet, rather than the two-inch exposure over as much as two hours that many others practiced (New York Times; Kevles 67). The difference in distance and time may be why Dr. Morton's images lacked the clarity and detail provided in images by some of his contemporaries.

While the potential dangers of X-rays were not immediately apparent, they became clear within the first few years. Thomas Edison, inventor and capitalist, saw the medical and commercial potential for X-rays and began experimenting with them within hours of hearing of Röntgen's discovery (Kevles 34). Within months, inventors and technicians began reporting hair loss and skin irritation from X-ray exposure; Edison himself suffered from skin rash and eye irritation, so he abandoned the work by 1902 (Kevles 38). Unfortunately, his chief assistant, Clarence Dally, who often held items for Edison to X-ray, suffered severely from the work; initially, he experienced skin irritation and hair loss, but his skin was so damaged by radiation burns, that amputation was required for his fingers, hands, and parts of his arms, until he died painfully in 1904 at age 39 (Cartwright 110). Dally's case was not unique. By 1911, at least fifty radiologists had suffered serious health consequences, or death, from radiation over-exposure causing burns or leukemia (others died from electrocution due to faulty electrical cables) (Kevles $48,61,89)$. Protective equipment was marketed to radiologists as early as 1907 , and by the 1920s, discussions began in the radiological community to determine safe levels of radiation exposure (Kevles 56, 89-92). In 1924, a "tolerance dose" of X-rays was established in the U.S.; in 1931, this dose was accepted as the international standard (Kevles 306, 308). In spite of the serious potential consequences of X-ray exposure, the public remained largely unconcerned. 
Beyond pure curiosity, the public was quick to see potential medical and legal uses for Xrays. People injured by bullets, for instance, insisted that they be X-rayed so that the precise location of the foreign object could be determined for surgical removal, even when the material had been embedded in their flesh for years without issue. While some patients experienced miraculous recoveries, in this pre-antibiotic era, some patients also died from surgical complications (Dam 408; Kevles 30; Reiser, MRT 61-62). X-rays were taken of broken bones, recently set bones, post-surgical patients, and to discover the skeletal structure underlying malformations (Dam 408). ${ }^{66}$ They were first used as evidence in court in 1896, in Canada, the U.S., and U.K., and in 1897, X-rays helped identify the dead from the Bazar de la Charité fire in Paris ${ }^{67}$ through dental records (Kevles 305). James Smith used an X-ray to prove that his doctor had improperly treated his broken hip (Kevles 31); one man used an X-ray to prove that a doctor had failed to properly set his broken thumb (Dam 419). While X-rays could provide proof of malpractice, they were also subject to interpretation, and sometimes misrepresentation, in court. In 1897, Harvey R. Reed suggested that there was a "halo of uncertainty" in X-ray images; foreign bodies, such as bullets, might escape imaging, and yet be locatable surgically. Images might be misinterpreted due to shadows, magnification, or distortion (Kevles 93). Kevles notes that, "Early X-rays had...been confusing, even to doctors who were supposed to be familiar with anatomy" (229). Yet physicians, notably surgeons, became so concerned about the possibility of malpractice suits, that they began taking pre- and post-surgical X-rays as proof that they had

\footnotetext{
66 "Beneficial" side effects were also discovered: X-rays cured acne; in 1904, separate experiments in Boston and San Francisco succeeded in bleaching a black man so that he appeared white; X-rays were also an option for sterilizing individuals deemed eugenically unfit for the gene pool (Kevles 49, 123, 311).

${ }^{67}$ First held in 1885, this annual charity bazaar was popular among the Parisian aristocracy. The 1897 event was held in a large mock Medieval-style building constructed of wood, cardboard, and fabric. An early form of movie projector was a special attraction that year; unfortunately, on May 4, it caught fire. The ensuing blaze killed 126 people, and seriously injured 250. Our Lady of Consolation Memorial Chapel was built to commemorate the dead (Bazar de la charité).
} 
performed the necessary operation, even when such images were not medically necessary (Reiser, MRT 66-67).

If the anatomizing capability of the stethoscope tended to objectify patients, X-rays extended patient objectification to a new level. While physicians did not, perhaps, suggest that X-rays should replace established hands-on examination techniques, or that X-rays should reduce physician contact with patients, the new imaging technology did have these effects. Previously, bones were set by palpation; bullets or other foreign material were removed through painful exploratory surgery (Reiser, MRT 63-64). As with other medical technologies, X-ray images effectively removed vital information from the patient so it could be examined and discussed objectively by a group of physicians in consultation (Benedict in Reisser, $M R T$ 79). X-rays also rendered earlier technologies, like the stethoscope, redundant. Why try to imagine what a patient's interior looked like using sound, when an X-ray could provide an actual picture? In some cases, for instance lung disease, X-rays might provide earlier diagnosis than either auscultation with a stethoscope or hands-on percussion (Reisser MRT 65).

In an attempt to make the soft tissues visible to X-rays, researchers soon began experimenting with contrast agents, such as bismuth and barium for the digestive tract (Kevles 70). Images taken with bismuth helped correct long-held positional anatomical errors; for instance, the cadaveric digestive tract lies differently from that of a living patient (Kevles 67; Reiser, MRT 64-65). As already discussed, much of medicine relies on the anatomy of the dead, but this information may not always map correctly onto the living patient. Anatomical atlases not only flatten the three-dimensional body, but they also subtract the vitality of the living body, which may lead to slight variations in biology, notably in the organs. In 1919, air was introduced as a successful, but painful, contrast agent for the brain and spine; in 1921 lipiodol, an oil 
suspension of iodine, was accidentally discovered as an effective contrast medium for the spinal nerves (Kevles 100, 103). The first angiogram X-ray image was of the cerebral, rather than cardiac, arteries, and was announced at a neurological meeting in 1927 by Egaz Moniz. ${ }^{68}$ Moniz experimented with a variety of contrast agents, introducing them into the carotid artery, until iodine became the medium of choice. The first successful cardiac angiogram was performed in 1929, through self-experimentation by Werner Forssmann, using an iodine-based contrast medium. Forssmann's innovation was to use a catheter to introduce the contrast medium to the required location (Kevles 105-107). Contrast agents were used for both still X-ray and moving fluoroscopic images.

About a month after Röntgen's discovery, the fluoroscope was developed. Although never as medically popular as X-rays, it is still used for viewing the digestive tract in action. The fluoroscope's transient images, while instantly available, do not provide a permanent comparative record. As with some other instruments, the fluoroscope provides an extension of an individual's viewing range, but cannot be shared with colleagues in the manner of an X-ray (Reiser, $M R T$ 62-63). Fluoroscopes also require longer exposure times at higher X-ray doses, so they pose greater radiation risks to patients, and physicians or technicians, than do still X-rays. ${ }^{69}$

\footnotetext{
${ }^{68}$ Moniz is a controversial figure, best known for winning the Nobel Prize for medicine in 1949 for inventing the frontal lobotomy (Kevles 105-106).

${ }^{69}$ The most popular use of fluoroscopes was not medical, but in shoe stores from the 1920s to 1950s. Advertising campaigns targeted parents wanting to ensure that their children's shoes were correctly fitted, so children were perhaps at greater risk than adults. The shoe-fitting units were composed of a cabinet with a slot at the base where the customer could insert his or her feet above the X-ray tube; three viewers at the top of the cabinet allowed the clerk, parent, and child to see the resultant images. Although exposures were relatively brief (up to forty-five seconds), the machines were not professionally installed, not regulated, and the clerks were not trained technicians. Although focused on the feet, radiation also leaked up customers' legs and pelvis, although no reports exist of customer injury from exposure. The sales clerks, subjected to repeated daily doses without protection, as well as radiation leaking from the machines into the store, did suffer health consequences, such as radiation dermatitis. A 1950 National Safety News article reported that a shoe model required leg amputation due to serious radiation burns (Bavley in Frame). By the mid-1940s, some legislative bodies began seeing the health consequences of prolonged and repeated exposure, and by the late 1950s, some states banned shoe-store fluoroscopes. Although never expressly banned in Canada or the U.K., the machines gradually fell out of favour and disappeared by about 1970 (Frame).
} 
During the first half of the twentieth century, the routine use of medical X-rays increased, with the unfortunate side effect that patients might receive five to ten times more X-rays than were diagnostically necessary (Reiser, $M R T$ fn, 160). While touring American hospitals in 1912, a visiting French physician commented, "Wherever I went I saw nothing but X-ray negative plates...” (Austin 802). Between 1920 and 1950, the number of X-rays taken in the U.K. doubled every five years; from 1938 to 1958 , there was a six-fold increase in the number of X-rays taken in American hospitals. A 1971 study indicated that for every sixteen X-rays taken, only one fracture was diagnosed, and that about one third of X-rays were unnecessary (Reiser, MRT 160). $\mathrm{X}$-rays, and other routine testing, revealed little useful information, duplicated existing information from patient records, and placed patients in potential harm from excessive radiation exposure (Reiser MRT 161).

Foetal X-rays, first developed in 1926, were potentially lethal to both mother and infant; they required an injection of carbon dioxide gas into the womb, and an exposure of about one hour due to the substantial mass being penetrated (Weart in Kevles 230). By the 1930s, and with some improvements, foetal X-rays became routine. The practice continued until Alice Stewart published "Malignant Disease in Childhood and Diagnostic Irradiation in Utero" in The Lancet, in 1956. Her study co-related foetal X-ray exposure to childhood cancer deaths (Kevles 230). Foetal X-ray imaging was replaced by ultrasound, a non-radioactive imaging type.

Women's breasts were another X-ray target, but early imaging attempts were unsuccessful. Paul Leborgne's 1949 idea of compressing the breast to improve image quality was the first of many improvements in mammography, with the first successful mammogram performed in 1960 (Kevles 254, 310). A randomized study in New York from 1963 to 1966 indicated that routine breast screening decreased mortality rates (Kevles 253-254). Since the 
1980s, mammogram radiation levels have been reduced by 50\% (Kevles 257). Susan Love, a surgeon, indicates that the dangers of missing a lesion or carcinoma in its early, more treatable, stages, are far greater than the risk of radiation-induced cancer from screening. She cites that the level of exposure required to cause cancer is about 8,000 rads, ${ }^{70}$ while a mammogram is only about one-quarter of a rad (Love in Kevles 254). On the other hand, a recently published summary by the American National Cancer Institute, states:

Screening may not help you if you have fast-growing breast cancer or if it has already spread to other places in your body. Also, some breast cancers found on a screening mammogram may never cause symptoms or become life-threatening.

When such cancers are found, treatment would not help you live longer and may instead cause serious side effects (NCI).

The guidelines for breast cancer screening continue to change and for now, routine mammography remains a somewhat contentious issue.

In the late 1950s and early 1960s, X-rays were combined to render three-dimensional images with the development of computed tomography, also known as CAT or CT scanners. CTs create a series of cross-sectional slices, or tomographs, making it possible for a computer to construct an accurate three-dimensional map of the imaging site, allowing greater accuracy and detail regarding the size and location of abnormal tissue (Reiser 161; Vaughn 25; see diagram in Brenner and Hall 2279). Due to the algorithmic complexity of mapping an interior density to render a three-dimensional image, it was impossible to build a successful CT prototype until advanced computer technology was available (Kevles 147, 24). The first viable CT scanner was introduced by Godfrey Newbold Hounsfield of the EMI Central Research Laboratories in the

\footnotetext{
${ }^{70} \mathrm{~A}$ rad is the amount of radiation energy absorbed per kilogram. Rads have been superseded by grays (Gy) and milligrays (mGy) as the units of measure for radiation, where $1 \mathrm{rad}=0.01 \mathrm{~Gy}$ (Brenner and Hall 2278).
} 
U.K. in 1971, and published in 1972 (Kevles 155; Porter, CHM 208; Reiser 161). The first CT scan was for a suspected brain tumour; taking X-ray images through a $180^{\circ}$ arc over fifteen hours, 28,000 images were captured on magnetic tape, which was manually transferred to a computer in another part of London for processing, then transferred to a second computer for image translation to a monitor where it was photographed, so the resultant image could be returned to the waiting physician and patient across town. The tumour was located and successfully removed surgically (Kevles 159-160). As computers have advanced since the 1970s, so has CT technology, rendering higher resolution images much more efficiently.

Unfortunately, with every new technology, there may also be drawbacks, and CT was no exception. While physicians and radiologists had become fairly comfortable reading flat X-rays, CT images were considerably more complicated (Kevles 162). More complex images required more advanced interpretive skills, and yet by 1980, almost every hospital had a CT scanner, and their use has more than tripled since 1993 (Kevles 188; Berrington de González et al. 2071). CT machines cost at least $\$ 100,000$, so they strained hospital budgets. Kevles suggests that, at least in private healthcare jurisdictions, like the U.S., unnecessary scans were likely ordered to help pay for the machines.

Because CT scanners multiply the number of X-ray images taken, they also subject patients to many times the amount of radiation of regular X-rays (Brenner and Hall 2278). A study published by Baumann et al. in 2011, suggests that undergoing two or three CT examinations in a lifetime is roughly equivalent to the radiation exposure experienced by a survivor of Hiroshima, yet few patients are aware of this risk. Using convenience sampling in a hospital emergency room, 1,168 patients presenting with acute, non-traumatic abdominal pain were recruited for this study. Baumann et al. found that patient confidence in their diagnosis 
increased fourfold when CT imaging and lab work were added to a physical examination and history-taking (6). The study also found that nearly $75 \%$ of patients underestimated CT radiation levels in comparison to chest X-rays; fifty participants even expressed confusion about the question, thinking that $\mathrm{CT}$ radiation exposure was lower than X-ray radiation exposure (Baumann et al. 4). Only 3\% of patients agreed with the statement that CT exposure over life could increase cancer risk. This lack of patient knowledge is particularly unsettling when further investigation indicated that over $80 \%$ of the study's patients had already received CT scans in the previous twenty-two years. Of the study sample, $53 \%$ had received more than five scans; one individual had received fifty-seven CT scans (Baumann et al. 6)!

Another study showed that although the number of emergency and hospital admissions remained fairly consistent, the use of CT scans rose from 6\% to 15\% between 1998 and 2007 in American hospitals (Korley et al. 1465). A 2007 American study suggested that 1.5\% to 2\% of cancers are likely caused by radiation overexposure from CT scans (Brenner and Hall 2282). Still another study extrapolates that, based on current levels of CT use, $1 \%$ to $3 \%$ of future cancers, or 29,000 cases in the U.S., will be caused by CT radiation exposure in 2007, estimating that half of these will result in death. The cancer risks from CT radiation overexposure are slightly higher for women; most prevalent in patients having chest, abdomen, whole-body, or CT angiogram examinations; and increase the younger a patient is at the time of exposure (Berrington de González et al. 2071-2073).

The advent of CT further increased the stress on patient-physician communication. Reiser notes a tendency for physicians to "cut short their customary diagnostic examination when using it [CT]" (161); or, at the extreme, "Sometimes we send people off for a CT scan before we talk to them" (Dr. Brian Hodges, November 23, 2012, Taking Toronto's Healthcare History 
Conference, University of Toronto). Again, there seems to be a co-relation between increased dependence on advanced technology, and decreased communication with patients; yet again, patients have colluded with physicians to create this potentially dangerous situation. As Baumann et al. conclude, "patients' confidence levels in their medical evaluation increased with increasing use of technology, with the inclusion of CT yielding the highest degree of patient confidence in a medical evaluation" (7). Patients seem to trust technologically supported diagnostics more than they do the training and experience of their physicians.

While all previously discussed imaging technologies rely on the transmission of radioactivity into the living body, positron emission tomography, or PET, reverses that notion, using the scanner to receive radioactive emissions from the body. PET scanners create images by monitoring the decay of a radioactive tracer injected into the patient's body. The first PET scanner was built in 1951 by George Hevesat self-experimenting with deuterium. PET scanners became viable in 1975, again, once computer technology was adequately advanced, and with the discovery that the algorithms used to acquire CT images would also render PET images. PET scans proved useful for brain imaging, registering metabolic changes apparent in Alzheimer's disease, depression and anxiety disorders, schizophrenia, and more (Kevles 202, 204). Since the 1990s, PET scans have also been used for cancer detection, often before physiological changes can be appreciated in other imaging types (Kevles 211).

Although a productive diagnostic tool, PET scanners have also proven controversial due to the safety debate about introducing radioactive material into the patient's body (Kevles 224). In the U.S., the Federal Drug Agency listed PET scanners as experimental until 1995, when they were finally approved for clinical use (Kevles 317). Another major issue for PET scanners is the cost. Each installation requires the PET scanner itself, a cyclotron to produce the necessary 
radioactive material, and three specially trained staff members; one to run the scanner, one to run the cyclotron, and one to handle the radioactive material. The scanner and the cyclotron each costs as much as a single MRI unit, effectively doubling the machine's cost; this is, however, offset by the fact that a PET scan only takes five minutes, compared with an average time of an hour for an MRI (Kevles 223-224). As with other advanced images, "PET is an enigma to the untrained eye" (Kevles 227). The monochromatic images show outlines, with splotchy patches of black, although these are often colourized to highlight areas of activity. Again, without skilled interpretation, PET scans mean very little.

While computer assistance improved imaging, not all medical imaging developed during the twentieth century required radiation; sound and electromagnetic imaging technologies were also invented. Ultrasound, the use of high-frequency sound waves for detecting mass, was developed during World War I to locate enemy submarines. In 1937, Karl and Friedrich Dussik, brothers who were a neurologist and a physicist respectively, attempted ultrasonic imaging on a patient's brain; however, the skull proved too thick to penetrate (Kevles 234). With further development, by the 1940s ultrasound could capture diagnostic images, and by the 1960s, it was generally accepted and commercialized (Orenstein 28). ${ }^{71}$ In 1957, John Wild accidentally discovered that ultrasound could detect cancers, and by 1968, Elizabeth Kelly-Fry had adapted ultrasound technology for mammography (Kevles 238, 235). Ian Donald, Chair of Midwifery at Glasgow University, championed foetal ultrasound, publishing "Investigation of Abdominal Masses by Pulsed Ultrasound" in The Lancet in 1958. In the 1960s, ultrasound was used in highrisk obstetrical cases, but by the 1970 s, foetal ultrasound had become routine; as most foetal

\footnotetext{
${ }^{71}$ In 2010, General Electric began marketing the Vscan ${ }^{\mathrm{TM}}$, a hand-held ultrasound device for use in remote areas where full-sized ultrasound technology is not available. For an evaluation of both the technology and its promotion, see my chapter, "The Message in Medical Imaging Media: An Analysis of GE Healthcare's Vscan TM" in Finding McLuhan: The Man/The Mind/The Message (Regina: University of Regina Press, 2015)
} 
ultrasounds are not medically necessary, it remains controversial (Kevles 243, 249). Real-time imaging became possible once computers were integrated with ultrasound technology in the late 1970s. Ultrasound and X-ray machines have similar costs, about one-quarter of the price of a CT scanner, and one-eighth of the cost of an MRI machine (Kevles 244).

Magnetic resonance imaging, or MRI, uses magnetic fields to alter the alignment of the body's atoms, rendering three-dimensional images of the soft tissues, largely excluding the bones (Carr 83; Kevles 173, 197). An MRI imaging session begins with the machine dividing the section to be examined into slices, measuring the activity of the hydrogen atoms in each section; this quantitative data is fed back to the computer to recreate the image (Joyce 439). Although the physics necessary for MRIs was conceived in the 1920s, the first application of MRI to living organisms (mice) was in 1959, and the machines were not common in medical use until the late 1970s (Carr 83; Kevles 177). As with other advanced scanners that digitally reconstruct the body's interior, MRIs required advanced computer algorithms to function. In 1972, Raymond Damadian discovered that MRIs could detect cancers, and in 1977, he unveiled the first full-body tube MRI machine (Kevles 179, 314). Similar to CT scanners, MRIs are large and expensive; MRI equipment can cost $\$ 1,000,000$ or more. The integration of MRIs into hospitals was slow, perhaps because they entered the market when hospitals were still paying for their new CT equipment. Furthermore, physicians, spoiled by the advanced imaging capabilities of CT scanners, found MRI images lacked clarity (Kevles 188). MRIs have relatively few user drawbacks. The primary concern is that metal implants or particles embedded in the patient's body are subjected to a strong magnetic pull, and will be attracted out of the body, damaging any impeding tissue (Kevles 191). 
According to a 2011 report in The New York Times, however, MRIs are often overused, with negligible clinical effect. Dr. Sangeorzan, one of the doctors interviewed, said, "An M.R.I. is...a very sensitive tool, but it is not very specific." Most MRIs will indicate some abnormality, but most abnormalities are of no clinical consequence. A study by Dr. DiGiovanni at Brown University concluded that $90 \%$ of MRIs are unnecessary and that $50 \%$ of MRI interpretations are either incorrect or inconsequential. He also observed that, "Patients often feel like they are getting better care if people are ordering fancy tests, and there are some patients who come in demanding an M.R.I.” (Kolata). Once again, patients, as well as physicians, drive the demand for more, and sometimes unnecessary, imaging.

Increasingly since the 1970s, imaging has become foundational for Western medical diagnostics. According to the Canadian Institute for Health Information (CIHI), in 2011-12, Canadians received 4.4 million CT scans, and 1.7 million MRIs, more than doubling the numbers from 2003-04. The 510 CT scanners across the country are kept busiest in New Brunswick, where 209 people out of every 1000 were scanned in 2011-12. Canada's 308 MRI machines are kept busiest in Ontario, where 61 of every 1000 people received an examination in 2011-12. Nationally, 126 of every 1000 people had CT scans, and 49 of every 1000 people had MRIs. This places Canada near the middle of imaging use internationally: CTs range from a low of 50 per 1000 in Chile, to a high of 275 per 1000 in Estonia; MRIs range from only 7 per 1000 people in Chile, to 98 per 1000 people in the United States (CIHI). Complementary imaging types have recently been merged to create fuller pictures, enhancing data collection, while only subjecting the patient to a single imaging session (Kevles 227). In 2000, PET and CT scanners were combined, and in 2008, prototypes for PET and MRI combinations were engineered (Beyer et al. 1369; Catana et al. 3705). PET or PET/CT scans were performed on 62,668 Canadian in 2011-12 
(CIHI). More complex technologies require more training for both the operating technicians and the interpreting radiologists. As a spokesperson for the Radiological Society of North America stated in 1985, “...the difference between using MRI and conventional X-ray techniques, including computed tomography CT scans, is like the vast difference between operating a 747 jet and an automobile" (cited in Kevles 222-223). While medical imaging holds clear patient benefits, mediation by advanced technologies also troubles the patient-practitioner relationship.

\section{Troubling Images and Imaging}

I joke, but only half joke, that if you show up in an American hospital missing a finger, no one will believe you until they get a CAT scan, MRI and orthopedic consult.

\section{Dr. Abraham Verghese (PBS)}

With the aim of improving patient outcomes, imaging technologies enable physicians to diagnose health problems efficiently, helping to guide treatment and surgical procedures. As Kevles notes, however, "While there is general agreement that imaging accelerates early detection, there is a good deal of disagreement over whether early detection makes any difference in terms of deaths from disease" (258). In spite of this tension, both patients and physicians engage with medical imaging fairly uncritically. Journalist Malcolm Gladwell comments, "there are few cultural reflexes more deeply ingrained than the idea that a picture has the weight of truth" (1); to which Blaxter adds, "A picture provided by a machine carries with it a sense of objectivity and authority" (CVP 772).

Yet to accept medical imaging uncritically is to disregard the subjective element introduced by the physician's interpretation of images, the patient's experience as an object of 
medical imaging, as well as the necessary communication between physicians and patients regarding medical images. In The Transparent Body, professor of media studies José van Dijck comments that, "the myth of total transparency generally rests on two underlying assumptions: the idea that seeing is curing and the idea that peering into the body is an innocent activity, which has no consequences" (6-7). The popular notion is that more finely tuned technological instruments lead to deeper and more accurate information; from a patient perspective, better technology means more insightful diagnosis, which translates into faster treatment, higher rates of cure, and almost guaranteed survival. Some patients expect physicians to save them and cure them, no matter what ails them, no matter how far advanced. This unrealistic expectation creates unreasonable pressure on physicians, and can lead to catastrophic disappointment when the physician is unable to deliver.

Interpreting the visual sense has been, and continues to be, problematic for the medical understanding of images. Recently, errors in imaging interpretation have caused some Canadian patients to lose faith in the medical system. According to a $\mathrm{CBC}$ investigative report into imaging mistakes, in 2010, the cancer that patient John Moser thought he had was ruled out through imaging; when his cancer diagnosis finally did come to light, it was terminal, and he died in 2011. A B.C. Patient Safety and Quality Council investigation found that none of the four radiologists in Moser's case was qualified. In Quebec, 109 breast cancer cases were missed between 2008 and 2010, with similar problems in other provinces. While these issues are sometimes attributed to radiologists being overworked, or their age leading to diminished visual acuity, Doug Cochrane, Chair of the B.C. Patient Safety and Quality Council comments that, "The technology in some ways has outstripped our ability to learn on the job" (CBC). The importance of the accurate interpretation of medical imaging becomes paramount when it is a 
primary diagnostic tool; these are, after all, what Waldby calls "operative images," images upon which decisions are made that "materially order the living body," that transform human bodies and human lives $(27,109)$.

Concerns regarding accurate X-ray interpretation began as early as the 1923 publication of Hugh J. Mean's, “The uses and limitations of the X-ray” in the Ohio State Medical Journal. Articles of this period raise concerns about technical problems, lack of knowledge about human variation, subjective differences in radiologists' visual accuity, the separation of the radiologist from the patient, and a blind belief in the perfection of X-ray diagnostics (Reiser MRT 189). Regarding some physicians' beliefs in X-rays, in 1928, Evarts Graham writes, “...presto! There is the diagnosis all but labelled for him on the film. Like the pink ticket of the fortune-telling machine!" (2).

A significant turning point in the critical analysis of X-ray interpretation was an accidental finding in Carl Birkelo et al.'s 1947 study, “Tuberculosis Case Finding: A Comparison of the Effectiveness of Various Roentgenographic and Photofluorographic Methods." To the researchers' surprise, when five specialists reviewed 1,256 X-rays, their interpretations did not always agree; colleagues' assessments differed in about one-third of cases, and even a second viewing by the same specialist produced different findings in about one-fifth of cases (Birkelo et al. 365). The startling finding prompted an international study, in which three reputed radiologists read $100 \mathrm{X}$-ray films twice; this study confirmed Birkelo et al.'s results (Reiser MRT 190). A 1952 Danish study employed three specialists who found they agreed on the interpretation of only $12 \%$ of the 2,500 films they reviewed. Between them, about one-third of the pathological lesions were missed (Groth-Petersen, Løvgreen, and Thillemann). Also in 1952, A.L. Cochrane and L. Henry Garland published, "Observer error in the interpretation of 
chest films: an international investigation" in the British medical journal The Lancet. Garland, and others, published articles on both sides of the Atlantic well into the 1960s examining problems of interpretation and observer error in X-rays, all with similar results (Reiser $M R T$ 190). Birkelo et al. concluded their study with the recommendation that, "all survey films be read independently by at least two interpreters," a recommendation that has been echoed many times, but is still not generally practiced in Canada due to cost and the availability of trained radiologists (365).

More recently, Elizabeth Krupinski's 2000 survey article of chest, bone, and mammographic radiological studies indicates that on average, there is a false positive rate of $2 \%$ to $15 \%$, and a false negative rate of $20 \%$ to $30 \%$. Accounting for the false positives, Krupinski notes that overlying anatomical structures can be misconstrued; false negatives are more difficult to comprehend. While an area may seem abnormal, a radiologist may not perceive it as problematic, so it remains unreported (Krupinski 330). Krupinski also observes that the shift from viewing images as film on light boxes, to viewing images on computer monitors, has had a detrimental impact on radiological reporting; among the problems noted are a reduced viewing area, and decreased brightness, contrast, and resolution (332). ${ }^{72}$

Clearly human subjectivity enters into the accuracy and clarity of imaging data interpretation. While a radiologist's decision to mark something as suspicious or not is in part a matter of training and experience, van Dijck and physician James Potchen agree that in some part, it is also a matter of the radiologist's personality. Some individuals are more inclined towards risk, while others are more cautious; a radiologist's personal tendencies will be reflected in her or his work (van Dijck 7; Potchen 428). In an effort to avoid or reduce the subjective

\footnotetext{
${ }^{72}$ Additionally, poor software design sometimes causes radiologists to waste up to $20 \%$ of their time examining the search menu, rather than the image (Krupinski 333).
} 
interpretive component, Kevles notes that computer-aided radiography (CAD) became available in 1992. Krupinski suggests that CAD may help radiologists by drawing their attention and perception to neglected areas of an image, but also asserts that some lesions require humans for detection (331). While seen as a viable second opinion, the dual effect of CAD has been to make radiologists somewhat lazy in their own judgements, as well as a tendency to over-diagnose, to err on the side of caution (Nodine in Kevles 257-258). While over-diagnosing may be preferable to under-diagnosing, it contributes to unnecessary patient stress and cost.

In addition to studies of interpretive error, some researchers have explored the results of omitting or adding elements to images to determine professional perception. In a 2006 American study, radiologists with various levels of training were asked to separate sixty chest X-rays into two piles: normal and abnormal. In the study's second part, they were asked: "Is there anything on this film which, if not detected and reported, would adversely affect this patient?" (Potchen 424). Unbeknownst to the participants, some films were duplicated; surprisingly, $5 \%$ to $30 \%$ of the time, radiologists placed one image in the normal pile and its twin image in the abnormal pile. Also included in the set was one X-ray in which the left clavicle (collarbone) was obviously missing; 58\% to $60 \%$ of the radiologists classified the X-ray as normal (Potchen 425). Rather than subtracting something, Drew et al.'s recent study of "inattentional blindness" added something to a stack of CT images: a picture of a gorilla (1) ${ }^{73}$ A typical chest $\mathrm{CT}$ produces a stack of 100 to 500 images; the gorilla appeared in a stack of 239 slices. It was about the size of a matchbook, about forty-eight times larger than the nodules the radiologists were seeking, located near a nodule, and appearing from $50 \%$ to $100 \%$ resolution over five slides (Drew et al. 2).

\footnotetext{
73 The researchers acknowledge that the choice of a gorilla is a reference to the 1999 study by Daniel Simons and Christopher Chabris in which viewers were asked to count the number of times a basketball is passed, distracting them from the person in a gorilla suit walking through the field of play (2). While this experiment in selective attention has been repeated and used in other contexts (a British cycling awareness campaign, for instance), the original video is still available online: http://www.theinvisiblegorilla.com/videos.html
} 
Although they detected $55 \%$ of the nodules, the anomaly was missed by $83 \%$ of the radiologists, some of whom looked at it directly (Drew et al. 3, 5). While it is easy to see the fun in such studies, the more serious implication is that even professionals trained to critically view such images often miss or dismiss the unexpected. The results of such blindness are unclear for patients, but they must be considered.

Compared with X-rays, mammograms present even more pronounced interpretive difficulties. Kevles cites that, "Mammograms are read by radiologists who miss a full 15 percent of malignancies" (257). As with all imaging types, while the technologies for capturing information continue to improve, the humans interpreting the data remain fallible and subjective. Problems can occur due to eye fatigue, eye disease, or limited training, but also because abnormalities can be difficult to spot in dense breast tissue (Kevles 257). Gladwell cites a study conducted at the University of Washington Harborview Medical Center, in which ten certified radiologists, examining the same 150 mammograms, interpreted them differently. Of the sample images, $18 \%$ were from women with confirmed breast cancers, yet one radiologist found masses suggestive of cancer in $78 \%$ of the images. Agreeing with van Dijck and Potchen, Gladwell notes, "Some radiologists see something ambiguous and are comfortable calling it normal. Others see something ambiguous and get suspicious" (Gladwell 4). Following the initial image examination, one radiologist had identified $85 \%$ of the cancer patients, while another had identified only $37 \%$. The radiologist who found the highest number of suspicious images also recommended the most follow-ups, including, "a biopsy, an ultrasound, or additional X-rays—on sixty-four per cent of the women who didn't have cancer" (Gladwell 4). A 2012 study based on ten years of data from the Norwegian Breast Cancer Screening Program, concluded that for every 2,500 women who undergo mammography, twenty women will be diagnosed with breast 
cancer, and one death from breast cancer will be prevented. Additionally, between six and ten woman will be overdiagnosed; they will be treated for breast cancer, although their cancers are not clinically significant, and would not become so in their lifetimes (Kalager et al. 498). While we can only speculate on the anxiety these healthy patients might experience, as well as their physical discomfort, and the potential financial burden (depending on their healthcare jurisdiction), they certainly would experience some level of avoidable distress.

Beyond X-ray imaging, more complex digital imaging types introduce the additional interpretive problem of artefacts, imaging effects accidentally created by the technology itself. MRI artefacts may appear as black or white spots, duplicated or blurred images, or wavy lines (Joyce 448). Pauwels notes that medical imaging creates pictures of things we cannot perceive through direct observation; we rely on and trust the technology to take accurate pictures (150). The only ways to verify that the images are a correct translation of the body's interior are to repeat the imaging session or surgically open the patient. Professional trust and authority for observing the area of concern are transfered to the machine. An artefact, digital noise, something the machine accidentally produces, might be misinterpreted by a radiologist as an object of medical concern (Joyce 451; Pauwels 153). Prasad notes that even slight patient movement during an imaging session can produce artefacts (294). "Cross-talk," interference caused by tomographic slices that are too close together, appears as white dots on the image and can be interpreted as either an artefact or pathology (Joyce 448); the impact on the patient depends on the radiologist's interpretation of the digital error.

Setting aside such technical aberrations, medical sociologist Kelly Joyce further examines the impact of MRIs on notions of authoritative knowledge and the physician construction of patients. Through an ethnographic analysis of imaging sites and interviews with 
physicians and technicians, Joyce discovers that the professionals producing and interpreting images, "equate the image with the physicial body... and authoritative knowledge" (437). In this conflation, the body and its image become interchangeable, with the image being perceived as a superior and neutral agent for expert knowledge production (Joyce 438, 439). A similar notion is reflected in genomics researcher Maud Radstake's work in real-time imaging, suggesting that images are more tangible and malleable to doctors than are patients' fleshy bodies (82). Joyce contends that, as with X-rays, MRI interpretation can be problematic; bodies are constructed as well or ill based on the interpretations of radiologists usually working in isolation from patients (448). One of the radiologists interviewed by sociologist Amit Prasad stated: "MRI images can give a perfect positive test but a perfect negative test is not possible," so clearly interpretation is paramount in deciding what images reveal and whether they indicate health problems (300). As with other imaging types, Prasad suggests that patients cooperate in co-creating the reality of MRI images, although such images risk erasing patients' physicality in the medical context (294).

Drawing on biotechnology theorist Donna Haraway, Prasad dubs the technological shift in the medical gaze as "cyborg visuality" (292). Prasad observes that MRIs, and by extension other imaging technologies using computer algorithms for image construction, do not actually involve seeing, but rather a conversion of mathematical sequences into "spatial maps of internal parts of the body" allowing "an almost unlimited extension of the medical gaze" (309). "Cyborg visuality" seems similar to what Radstake calls “black-boxing," referring to the unseen computer magic that renders images from complex mathematical data measured from the patient's body (27). Waldby suggests that scanning imaging technologies that use tomography, have a tendency for "calibrating living bodies according to the capacities of computer-generated space, and 
facilitating their surgical or orthopaedic reworking... through a linkage with data homologs" (45). Reference to such homologs, the accepted anatomical norms of the human body, creates what Prasad dubs the radiologist's "bifocal vision" (301); the radiologist has one eye on images of the patient's body, and the other eye on anatomical references.

Also alluding to Haraway, Waldby notes that the use of technology to reconfigure the subject body effectively blurs the historic line between human and machine, recasting the human body as purely informational (Waldby 46). The transformation of the subject into mathematical computer data requires the body's systematic fragmentation and dismemberment, working on the assumption that the whole is, like a machine, merely the sum of its component parts (Waldby 56, 66, 68). Visual culture scholar Lisa Cartwright suggests that contemporary medical imaging renders notions of bodily interior and exterior obsolete, and subsumes the body into "part of a living system that incorporates the technologies of its representation" (xiv). This kind of assertion is what leads sociologist Simon Williams to suggest that growing biotechnological control leads to a "moral, spiritual and existential crisis" of the corporeal body (1047). Because our bodies are increasingly plastic, can be rationalized and reconfigured almost at will, and are perceived as being at constant risk, the notion of the physical body becomes "ever more elusive and problematic" and strains to find meaning (Williams 1047). The blurring of lines between the human body and the technologically reproduced image supports Haraway's notion of the cyborg in current medical imaging practices.

While the imaging types previously discussed produce still images, real-time imaging, such as ultrasound, endoscopy, fluoroscopy, and angiography, raise unique problems. In Vision of Illness, Radstake examines the experience of real-time imaging, specifically considering the impact of imaging sessions on notions of patient embodiment. While acknowledging that 
"medical images show everything but one's own body," at least as we usually view it, and that imaging "alienates patients from their bodies," Radstake complicates the idea of a subject-object dichotomy in the patient's experience of real-time imaging (6). While agreeing that imaging is a form of mediation, she also suggests that during real-time imaging, patients relate to their bodies both subjectively and objectively, that during such imaging sessions "bodies cannot selfevidently be distinguished from their images" (Radstake 7, 6). Similarly to Joyce's previously discussed finding that physicians conflate the patient body with MRI images, Radstake suggests that in real-time imaging, patients conflate their own bodies with the images. Radstake argues that though image creation might objectify the patient, during real-time imaging patients are simultaneously aware of their subjectivity because of communication with the physician or technician, their visual perception of the images, their embodied haptic awareness of the process, their cooperation and agency as a participant in the imaging process, and sometimes even an emotional attachment to the images (92). Referring to patients' willing participation in preparing their bodies for the technology's requirements, Radstake borrows Gomart and Hennion's notion of "active dispossession" (56). Preparation might entail drinking water or a contrast medium like barium, or being injected with a contrast medium like iodine or a radioactive tracer. For Radstake, the moment of separation between subject and object body comes after the real-time imaging session, when still images taken from the session and the written report become fragmented data documents no longer attached to the individual patient's body, but interrogated in comparison with the norms and standards of anatomically healthy bodies (109).

With the first anatomical atlas, the three-dimensional subject body became flattened, rationalized into two dimensions. Cartwright suggests that this flattening of the corporeal was symptomatic of a broader cultural distaste for humanity's messiness, and a desire to move 
towards a clearer, more rational human being (91). While advanced imaging technologies, such as CT, attempt to revive the body's third dimension, they still "read the body's interior as digitised information configued on a computer screen" (Waldby 5). Although the body has been technologically reinflated, as it were, imaging still tends to create a sense of "medical objectification... which on the one hand augments and shores the status of the human, protecting subjects from the encroachments of diseased embodiment, and on the other generates its knowledges and procedures by treating the human as experimental object and passive biomass" (Waldby 7).

X-rays, ultrasounds, CT scans, and MRIs construct images of the body's interior and in so doing, they flaten, isolate, and objectify some part of the patient's anatomy. Each imaging type offers an "objective truth," yet may not reveal the same truth as an alternative imaging type; for example, an MRI may detect different information from an X-ray, and therefore may not construct the same patient or disease state. In Blaxter's case study, for instance, after a week of preparation, the patient's surgery was cancelled due to the interpretation of a PET scan, ignorning the patient's knowledge that what the concerning image revealed was an old injury. As Blaxter reports, "the world of the image and the world of the real body seemed to $\mathrm{P}$ to have existed in two separate spaces"(CVP 769). It is not that some imaging technologies are false, but rather that the technology has limitations, images are always subject to interpretation, and the subjective slippage between professionals within the field of image interpretation is surprisingly broad (Dershaw in Gladwell 11).

While interpretive error signals a serious problem in our trust of imaging technologies, another more subtle problem exists: the notion that imaging is a benign activity. While it might be less obviously invasive than picking up a scalpel, it is still invasive. There is a felt intrusion 
on and in one's person, a sort of intimate violation. The gaze penetrates with the aim of gathering information to choose whether or not to intervene more obviously; the meaning of this gaze is that it has the potential to change the structure of the body into which it penetrates (Lehoux 45, 56; van Dijck 7; Waldby 27). Advanced types of imaging, such as CT, PET, and MRI are more obviously intrusive; CTs may require the injection of a contrast medium, PETs require the injection of a radioactive tracer, and MRIs actively affect the body's atoms (Waldby 107).

Drawing on Foucault, Prasad suggests that by attempting precise answers, the medical community seeks "to discipline the images and through that the human body," a sentiment echoed by Cartwright (Prasad 301; Cartwright 108). "Disciplined" or "domesticated" images are, for Prasad, those presented and labelled in various types of anatomical atlases, as well as those presented in medical reports after they have been measured against these norms for signs of pathology (301). Bodies that deviate too much from these norms are considered pathological; the medical professional's role is to discipline such bodies through intervention so that they more closely meet standardized notions of human norms. Prasad also notes, however, that the norms used for reference in digital imaging, are anatomical norms rendered from an earlier medical gaze, one constructed using cadavers, rather than digital tomographs (303). To be tamed, to be rendered into a clear, coherent, and unambiguous report, images must be interpreted, organized, and labelled (Prasad 303-305); as Potchen asserts, "The purpose of any diagnostic procedure is to diminish clinical uncertainty" (424). Prasad acknowledges that this as an ideal, however; human bodies vary greatly according to genetics, gender, geography, demographics, and age. As Blaxter observes, especially with older patients, imaging is likely to reveal numerous anomalies, and while these may deviate from the medical conception of the "normal" body, it does not necessarily mean that they are abnormal for a specific body, or that they impede function (CVP 
768). Nevertheless, building on the existing Foucauldian notion of the medical gaze, even images that are digital reconstructions of data, rather than empirically or mechanically collected data, retain and extend the power of the medical gaze (Prasad 310).

The rapid growth of imaging technologies since the 1970s has led to their entrenchment in the modern hospital as a sophisticated addendum to a reductive medical gaze. Blaxter, Joyce, Waldby, S. Williams, and patient narrative scholar Arthur Frank all contend that because the image seems to trump everything else in contemporary medical discourse, the medical gaze has slipped into Jean Baudrillard's third order of simulacra, the hyperreal, in which the simulation, in this case the medical image, is substituted for the real (Blaxter CVP 762; Joyce 437, 441; Waldby 97; Williams 1047; Frank "Twin Nightmares of the Medical Simulacrum” 83). Frank states that in this inverted world, "the image on the screen becomes the 'true' patient, of which the bedridden body is an imperfect replicant, less worthy of attention" (TNMS 83). Unlike Xrays, which use photographic technology, as Prasad astutely points out, more advanced digital imaging technologies render an image of which there is no "original copy," only algorithmic simulations with a variety of possible interpretations (304). Yet, if patients no longer trust their own subjective reality, they may become more reliant on doctors' interpretations of images to determine how they feel.

The technological extension that imaging affords the Foucauldian medical gaze is, however, complex, both objectifying and constructing the patient. The clinical gaze must create the patient as both object and subject, as Mol and Law suggest (87). Doctors sometimes seem unaware that a mediating biotechnological gaze can cause patients to feel objectified, to struggle to assert their subjectivity. More, better, and faster imaging technologies may not lead to improved healthcare relationships, and they clearly problematize an already complicated site of 
communication between patients and physicians. Researchers agree that increased or better technologies are not the solution for the challenges of patient-practitioner relationships (Blaxter CVP 776; Cochrane in CBC; Prasad 302). Patient-centred communication offers a way to bridge the gap between patient and practitioner, especially when such mediating technologies are used. The current direction of diagnostic medicine seems to indicate that in the not-too-distant future, physicians will more closely resemble computer technicians than healthcare practitioners, and the patient voice may be even further stifled. Rather than working to accommodate technological advances, perhaps physicians should refocus on building meaningful therapeutic relationships with their patients through improved communication.

\section{Conclusion}

Examining the development of medical technologies and imaging, the reliability of imaging data, and the impact of imaging on patient-practitioner dynamics, this chapter narrows the focus towards the field research. As is clear from these first two chapters, communicative tensions between patients and physicians are apparent from the outset of medicine, and continue throughout medical history. These complex communicative problems form part of the foundation and precedent for contemporary medical students and practitioners. The hope is that this dissertation prompts physicians to critically assess their communicative strategies, and gives patients permission to speak and question. Building on Simon Williams's assertion that, "lay voices should be the final arbiters in these broader theoretical debates concerning the role of medical technology," the following chapters discuss the methodology and findings of field research examining the impact of medical imaging on patient-practitioner communication (1048). 


\section{Chapter 3: Methodologies for Exploring Patient-Physician Communication}

To attend to the lived body is not to forsake the tools and learning that Cartesian medicine has provided. It is merely to refuse to grant this mechanical wisdom the status of ruling paradigm.

Drew Leder (31)

In the medical interaction, patients and physicians must find a common language that enables them to comprehend each other through the exchange of information, to diagnose, and generate mutually understood and agreed upon treatment options. Whether mediating imaging technologies enable or impede this discourse, they likely shift the communicative dynamic in some manner. What is the impact of this mediation on patient-centred communication? Do, or should, physicians adapt their discourse in the context of imaging technologies? While profound biomedical advances may improve life quality and expectancy, new communicative strategies could empower patients in an increasingly technologically dominated medical environment.

\section{Research on Patient-Physician Communication}

...medicine is a unique interaction between two individuals, the patient and the physician, involving permission and (hopefully) trust.

\section{David Wootton (21)}

Since the 1970s, Western clinical education and practise has acknowledged the patient-centred model, one in which the patient is considered as a subject, a complex being with a familial and social context, who is having an experience of illness. Also since the 1970s, however, the biomedical practice model has grown increasingly reliant on advanced technologies for diagnostics, monitoring, and treatment. Patient-centred communication and advanced biomedical 
technologies, which have evolved concurrently in the same communicative environment, create very different versions of patients. While patient-centred practice evokes a three-dimensional, thinking, acting, feeling subject, imaging technologies render a flattened data set that is readily objectified.

The relationship and communication between patients and physicians is complex, requiring a profound level of trust. The patient-practitioner relationship relies on social and negotiation skills, rather than on technical ability. Physicians anticipate that patients will behave in certain socially ascribed ways during the medical encounter to support an assumed patientpractitioner relationship. Regarding physical exams, medical instructor Dr. Danielle Ofri points out that, "There are few situations where we expect to disrobe and have our bodies touched by relative strangers."

In her paper, "Nothing Unusual is Happening," sociologist Joan P. Emerson observes a young woman, inexperienced with gynecological exams, refusing to cooperate with the anticipated "nothing unusual" stance required of the compliant patient. The patient refuses to comply with the requests of professionals on whose care she relies, making it very clear that for her, "something unusual" is indeed happening. The lack of therapeutic alliance between the patient and medical staff underscores the ways that patients usually collude with physicians to create a "nothing unusual" dialogue during medical encounters (J. Emerson 213-216). Emerson suggests that, "A patient may look upon his medical condition and the technical procedures it elicits as highly unusual events, while the staff is reassuringly nonchalant" (211). In this example, because the staff defines the patient's state as routine, the patient feels that her personal concerns are being dismissed. In his effort to convince the patient to comply, the physician alludes to "the standards of good medical practice," an authority beyond his own (J. Emerson 
216). The power dynamic is obvious in this scenario; physicians expect patients to behave in certain ways, and when patients behave differently, are noncompliant, and want to renegotiate their submissive role, they may find themselves labelled as "difficult" or "demanding." The anticipated relationship between patient and physician is based on a tacit understanding that the patient will do as she is told, so that the physician and other medical staff can do their jobs to help her as they see fit. The crucial element lacking in the encounter, Emerson reports, is any acknowledgement of the patient's fears; the professionals fail to negotiate the care needs of this particular patient to create a therapeutic alliance. This scenario provides an example of the kinds of things that can go wrong when patient-physician communication is sub-optimal. As Wootton asserts, "Medicine has often involved doing things to other people that you normally should not do-touching them, hurting them, cutting them open" (21). As patients, we give permission for this (sometimes rough) handling on the understanding that ultimately it will be for our benefit. Ideally, what should happen in a negotiating dialogue between patient and practitioner? Numerous physicians, and others, have researched patient-centred interviewing, the interviewing style taught at the University of Toronto School of Medicine, and other medical schools throughout North America and Europe. In his book How to Break Bad News, British-Canadian physician and comedian Robert Buckman details effective patient-centred communicative methods. He notes that patients routinely complain that physicians do not listen, and that they use jargon, or speak down to patients. On average, physicians interrupt patients' narratives within the first eighteen seconds of an interview, and patients rarely have the opportunity to talk for more than 150 seconds at a stretch (Buckman 41). Buckman notes that when physicians actively listen to their patients, satisfaction, a sense of competence in the physician, and treatment compliance all improve. Physicians who can adjust their vocabulary to each patient 
ensure that they are using intelligible jargon, nor are they being condescending (Buckman 42). Physician behaviours that Buckman suggests to elicit a successful therapeutic relationship include: using open questions to gather information about new areas of discussion and to ensure clear understanding of patients' concerns (closed questions have their place, but must be used appropriately); avoiding biased questions that steer patients to what might be perceived as the "right" or more desirable answer (which may not be truthful); facilitating dialogue through active listening, rather than interrupting patients; and repeating, reiterating, or paraphrasing elements of patients' narratives, or reflecting patients' narratives adding some analysis to the story (48-52). This combination of communication techniques promotes patients' narrative, ensuring that physicians understand the story before responding and moving towards diagnosis. The response should be factual or empathetic, rather than judgemental or aggressive; depending on the subject, sometimes silence is an appropriate response (Buckman 55-58). This idealized interaction is complicated by patients' increasing medical education, and the abundance of information at our disposal. Although physicians are trained professionals, patients may challenge their knowledge, or diagnoses, or request unnecessary tests.

Buckman was one of the authors of the Toronto consensus statement on doctor-patient communication. This brief, but groundbreaking, declaration begins with a survey of existing literature, and then declares what changes need to be implemented in medical education to improve patient-physician communication. Even in 1991, this statement concludes that: Sufficient data have now accumulated to prove that problems in doctor-patient communication are extremely common and adversely affect patient management. It has been repeatedly shown that the clinical skills needed to improve these problems can be taught and that the subsequent benefits to medical practice are 
demonstrable, feasible on a routine basis, and enduring... If current knowledge is now implemented in clinical practice, and if the priorities for research are addressed, there may be material improvement in the relationship between patient and doctor (Simpson et al. 1387).

The Toronto consensus statement begins by acknowledging that poor communication is a common problem in the patient-physician relationship, declaring that, "Most of the essential diagnostic information arises from the interview, and the physician's interpersonal skills also largely determine the patient's satisfaction and compliance and positively influence health outcomes" (Simpson et al. 1385). Citing findings from a 1979 study, the Toronto statement states that an alarming " $54 \%$ of patient complaints and $45 \%$ of patient concerns are not elicited by physicians;" half the time these problems include psychiatric issues (Simpson et al. 1385). Physicians' inability to communicate successfully with patients can lead to both formal and informal complaints. By using jargon, or making assumptions about what patients want to hear, physicians often fail to adequately educate their patients, and "The quality of clinical communication is related to positive health outcomes" (Simpson et al. 1385). Patient compliance and satisfaction are improved, and anxiety lessened, when patients feel heard and understand what is being communicated. To facilitate improved patient-physician communication, the consensus statement suggests that physicians allow patients to discuss their concerns, without interruption; as patient disclosure takes, on average, ninety seconds, at most two-and-a-half minutes, it does not significantly prolong the interview (Simpson et al. 1386). The appropriate use of open and closed questions, active listening, requesting clarification when needed, checking in and providing summaries to show understanding, providing clear explanation to educate patients, negotiating options, and providing appropriate empathy, together provide 
facilitation techniques that "positively affect the quality and quantity of information gathered" (Simpson et al. 1386). Through improved medical education regarding communication, the Toronto consensus statement advocates strengthening skills for physicians to gather data (including biopsychosocial information), form strong therapeutic relationships with patients, discuss sensitive or difficult issues, provide patient education through understandable information, as well as addressing necessary therapeutic skills development (Simpson et al. 1386).

As well as considering verbal exchanges, some researchers have examined the importance of non-verbal elements in human encounters. In Silent Messages, psychologist Albert Mehrabian defines nonverbal communication as including facial expression and body language, but also verbal communication style (e.g., rate, intonation, volume). Mehrabian's research indicates that successful communication is only $7 \%$ verbal, $38 \%$ paralinguistic, and $55 \%$ nonverbal cues. Clearly, Buckman's suggestion that physicians sit down when speaking to patients has a positive impact (46). A 1970 British study found that non-verbal cues provided by an actor, were 4.3 times more effective than verbal cues, with women being more responsive to nonverbal elements (Argyle et al. 222).

Physicians in practise, under strict time constraints, often complain that they simply do not have time for this "touchy-feely stuff," or that providing patient comfort and education is not their job and should be supplied by nurses or other healthcare professionals. What such physicians fail to realize is that, when well and professionally practised, patient-centred interviewing techniques are just as efficient as the physician-centred biomedical approach (Weston quoted in CPSO). The Toronto consensus asserts: "Beneficial clinical communication is feasible routinely in clinical practice and can be achieved during normal clinical encounters, 
without unduly prolonging them, provided that the clinician has learned the relevant techniques" (Simpson et al. 1385).

Part of patient-centred communication involves eliciting the patient's narrative; what stories does the patient tell about his or her experience of illness? In a brief how-to guide, Allan Peterkin, psychiatrist-physician and head of the Health, Arts and Humanities Program at the University of Toronto, provides practical tips for eliciting patient narratives that can provide additional, often valuable, information to physicians. His suggestions include: asking open questions, avoiding interrupting, using metaphors or defining key words to ensure understanding, being aware of body language, being aware of one's own assumptions, and finally, asking the patient: "What do you think is going on?" or "What's the one thing you haven't asked or told me?" (Peterkin 63-64). Together, these techniques allow physicians to develop a more complete understanding of their patients, the impact of illness, and patient motivators. Peterkin also suggests that physicians should, "View noncompliance as a blocked narrative, not as patient stubbornness" (63). While it is understandable that physicians may feel frustrated by noncompliant patients, listening to a patient's understanding or reasons for noncompliance may help the physician clarify the patient's understanding and improve compliance.

Since the publication of the Toronto consensus statement, Peterkin and other physicians have lent support to the practise of patient-centred communication, for a variety of beneficial reasons. For instance, Dr. Jerome Groopman states that, "Most incorrect diagnoses are due to physicians' misconceptions of their patients, not technical mistakes like a faulty lab test" (quoted in CPSO). Misdiagnosis can readily occur when physicians jump to conclusions before gathering adequate information from patients. Dr. W. Wayne Weston, Chair of the Advisory Committee for the Institute for Healthcare Communication in Canada, declares, "If we don't listen carefully 
and create a relationship where patients can speak their mind, we'll miss a lot. Our job is to help patients deal with the impact of illness on what matters to them—so we need to know what matters" (quoted in CPSO). Understanding the impact of illness on individual patients may help guide physicians to treatment options with which patients can be compliant. Dr. Joshua Tepper, President of Health Quality Ontario, states: "Ideally, the better job we do at the front end of patient engagement, the less we will have a need for an ombudsman at the back end" (quoted in Taylor). Improved patient-physician communication leads to stronger therapeutic relationships in which patients feel included in the diagnosis and treatment options, increasing their sense of autonomy, satisfaction, and trust in their physicians. When physicians can link treatment plans to patients' expectations, compliance improves, enabling physicians to treat patients to the best of their abilities, and improving patient care; improved patient-physician communication improves healthcare.

Although patient-centred care has been taught in medical schools for over thirty years, it still seems like an ideal that not all physicians achieve in practise. Numerous studies regarding the impact of patient-physician communication, and its effect on patient care, have been undertaken in the last few decades, with varying results. Referring to research mentioned in Chapter 1 of this dissertation, Dr. Ronald Epstein et al.'s 2005 research survey attempted to define and quantitatively measure patient-centred communication. Ultimately, this study acknowledged that communication is qualitative, and difficult to define or quantify; however, common traits of patient-centred communication included acknowledging the patient's perspective and psycho-social context; sharing the medical problem and treatment options to ensure patient understanding and agreement; and sharing responsibility and power regarding medical choices (Epstein et al. 1517). The study concludes that patient-centred communication 
is, "a multifaceted construct" with "an elusive transcontextual 'way of being' that defines the essence of PCC - a unifying principle" requiring physicians to be attentive, curious, flexible, and fully present for their patients (Epstein et al. 1525).

A 2007 Canadian study using Roter's Interaction Analysis System (RIAS) is perhaps more successful in developing a quantitative discourse analysis. This research tool assesses nine areas of discourse: closed and open questions, biomedical information, psychosocial information, social conversation, positive and negative talk, facilitation, and orientation ( $\mathrm{Li}$ et al. 422). Through the analysis of thirty-one consultations, Li et al. examined the asymmetry of patientphysician encounters, noting that physicians control these encounters by dominating the discourse, thus reducing patient satisfaction (418). Similarly to other studies, Li et al. found that although physicians and patients spoke similar numbers of words, physicians dominated the discourse by asking questions, intrusive and interruptive facilitation, and giving instructions, leaving patients to answer questions and with limited time to introduce their own concerns. The numerical results from Li et al.'s study indicate that physicians dominated with biomedical speech during $27 \%$ of the interview, and positive talk for $26 \%$ of the interview; physician facilitation expanded into 16\% of encounters, and closed questions occupied another 12\% (424). Patients governed little interview time, asking closed questions $1 \%$ of the time, and open questions $0.7 \%$ of the time; although patients attempted to advance psychosocial talk $17 \%$ of the time, physicians only acted on these prompts $3 \%$ of the time (Li et al. 424). Clearly, physicians and patients are not successfully sharing the conversation. In this study, physicians asked $89 \%$ of the, generally closed, questions, while patients asked only $11 \%$ of questions; the role of patients is perceived to be that of answering questions only ( $\mathrm{Li}$ et al. 429). As Li et al. conclude: "physicians are experts and patients are uninformed. Therefore, both parties may believe that 
physicians should be in control of the process and content of the medial consultation. Physicians offer their opinions and decisions and patients accept these decisions. This pattern of communication enhances physicians' power over patients" (429). As Li et al. and others assert, however, physician dominance may lead to premature diagnosis before patients can offer all the necessary information. This may in turn result in misdiagnosis, leading to unnecessary testing, medication, or hospitalization; added expense to the healthcare system; and poor quality healthcare for the patient. Physicians would better serve their patients by loosening their need to control interviews (Li et al. 429).

Diagnosis offers an important site of information sharing between patients and physicians. As Blaxter notes, the word "diagnosis" is both a noun and a verb; it is both a descriptive category of problem, and the process of coming to this conclusion (Blaxter, Diagnosis as Category and Process, 9). Diagnosis establishes generalized categories across patients, supporting statistical information across populations (e.g., studies of morbidity), and also the possibility of disease treatment (as opposed to patient treatment) (Blaxter, DCP, 10-11). Such rigid notions of diagnosis can leave little room for social disorders, such as family violence or economic stress, which may also impact both physical and psychosocial health. Diagnosis leads to action, either by the diagnosing physician, or in referral to another practitioner such as a specialist (Blaxter, DCP, 13).

Communication researcher Richard Street Jr. and Dr. Howard S. Gordon's 2006 study revealed that, depending on the diagnosis, patient-physician conversations differ, potentially impacting patient consent, outcomes, and satisfaction (217). At the same healthcare facility, two types of post-diagnostic encounters were examined: post-angiogram and lung cancer. The postangiogram encounters were five to ten minutes long, while lung cancer appointments were up to 
half-an-hour (Street and Gordon 218). Even after allowing for the time difference, researchers still found differences in patients' active participation, with lung cancer patients being more invested (Street and Gordon 218-219). Lung cancer patients contributed $40 \%$ of the discourse during their appointments, compared with the $24 \%$ contribution of post-angiogram patients (Street and Gordon 220); lung cancer patients were more assertive and directive of the conversation, while angiogram patients asked more questions (Street and Gordon 222). Street and Gordon conclude, not surprisingly, that short appointments reduce patient participation and enhance physician control over the discourse. Although physicians could improve patient participation through some of the communicative techniques outlined above, this may not be perceived as desirable due to time constraints (Street and Gordon 223).

As Prado et al. suggest in their 2013 survey article, "When physicians are uncomfortable with the truth disclosure process, they may avoid distressing information (bad prognosis, e.g.) or convey the news in an overly optimistic way" (15). This contention of "truthiness" in medical communication is supported by theologist Christopher Breitsameter's 2010 article indicating that treatment risks may be downplayed by physicians. Patients rely on physicians to inform them, to provide all the necessary information (benefits, risks, and alternatives) so that they can make informed decisions; however, Breitsameter charges that, "patients are not provided with complete and accurate information" (349). Clearly, this is another complex aspect of patientphysician communication. Physicians must explore each patient's ability to comprehend the information, and his or her emotional and intellectual competence to make appropriate decisions (the "appropriateness" of the decision usually meaning the decision that agrees with the physician's). With increasingly available information, patients may feel more confident to make decisions; however, the physician's experience must not be discounted. Breitsameter charges that 
treatment risk is often intentionally not communicated, even in the journals upon which physicians rely. He suggests that communicating the adverse affects of new treatments or medications may not serve the "interests of pharmaceutical companies in stressing the advantages of a new development" (Breitsameter 351). Even when claims are accurate, the way they are communicated can make them sound more beneficial than they are in practice. Using Breitsameter's example, stating a risk reduction of $50 \%$ is profound, and likely to lead to increased prescription; however, this might mean reducing the number of affected patients from four in 10,000 to two in 10,000, which sounds much less impressive (Breitsameter 351). Numbers may not lie, but they can certainly be manipulated to deliver the most fiscally desirable message.

While Breitsameter traces these omissions to pharmaceutical companies, medical anthropologist Sylvie Fainzang suggests other causes of lying between patients and physicians, and asserts that it is common practice in the patient-physician relationship. Fainzang asserts that "some doctors only give information to patients with the view to enabling the latter to make a decision which conforms to the doctor's opinion, and therefore to obtain their own therapeutic objectives" (37). She continues by suggesting that some physicians choose to avoid discussing adverse effects and actively discourage patients from reading this information (Fainzang 38). By refraining from disclosing information, physicians "claim the privilege of knowledge concerning the patient's body and tend not to disclose information that might enable the patient to make his own choices concerning his being" (Fainzang 46). Patients' lies are different; patients tend to lie about medication or treatment compliance. They may feel guilty for not trusting their doctors, or trying alternative treatments, and not wish to irk their physicians by sharing this information (Fainzang 39). In both cases, the types of information omitted from the discourse, as Fainzang 
suggests, further entrench the power dynamics of traditional patient-physician roles. While physicians lie through omission to retain power, patients lie to express resistance to physicians' power (Fainzang 46-47). As fictional TV physician Greg House was fond of saying, "Everybody lies."

Malpractice suits are levied when something goes horribly wrong medically, but as gastroenterologist John Petrini asserts, legal proceedings are less likely when patients have good communication and trusting relationships with their physicians, and feel well informed of potential risks of a procedure (382). Petrini suggests that,

The breakdown in the patient-physician relationship often underlies a malpractice claim. Communication is essential to establish a partnership between the patient and physician, so that a free exchange of information can be provided. Patients need to understand the reasons for a particular course of action and the ramifications for having or not having the suggested treatment. Alternative treatments or diagnostic tests must be discussed and evaluated. (383)

As will become apparent in the analysis of patient interviews in the following chapters, patients often feel that these dialogues do not happen in practise.

Ideally and ethically, medical decision-making should occur in negotiation between patients and physicians, thus ensuring that patients provide informed consent to treatment. In practise, the notion of informed consent seems somewhat malleable. One of the most difficult aspects of the physician's job is to deliver bad news, especially when it is not anticipated, yet in our cultural context this is the expected course of action when physicians discover an acute pathology. As Buckman suggests, in this instance the physician is privy to information of which the patient is ignorant. The interview's flow depends largely on the patient's reaction to the 
news. Buckman advises that the physician ensure a comfortable context, and the presence of additional patient support (family, friends, and/or professionals). The next two steps are crucial, yet sometimes overlooked: ask how much the patient already knows, and "Find out how much the patient wants to know" (Buckman 96). Being patient-centred means ascertaining each individual patient's needs; the best way to discover this is to ask. Some patients want all the details, opportunities to ask questions, access to informative resources, and a say in treatment decisions; other patients may not want to know anything, and may quite willingly defer to the physician's judgement for treatment. Either way, physicians must allow patients to be actively involved in the flow of information towards decision-making. An excess of information during a bad-news encounter is overwhelming, and will likely require repetition at a later appointment. Divulging the diagnosis is necessarily followed by a therapeutic dialogue in which the physician's primary responsibility is to respond to the patient's needs for information and psycho-emotional support. The interview should end with a summary and clarity for follow-up (Buckman 65-66).

Clearly, divulging bad news is physiologically and psycho-emotionally difficult for both physicians and patients, as explored in Ariadne Prado et al.'s survey article. Physicians and patients both experience increased heart rates and blood pressure, as well as spikes in cortisol levels and immune response (Prado et al. 15-16). Depending on their speciality, some physicians are more frequently faced with breaking bad news. Oncologists, for instance, may deliver bad news as many as thirty-five times per month, more than daily, in cases where palliative care is the only option. Stress from such encounters may last from hours to days, meaning that some specialists are constantly stressed. Not all bad news is the same; having to deliver news about palliative care or medical error is more difficult than delivering bad news about a patient's 
treatable diagnosis. Physicians are, predictably, prone to burnout due to emotional exhaustion, depersonalization, and a sense of limited achievement (Prado et al. 14). Psycho-emotionally, physicians are concerned with losing control of their professionalism and emotions (Prado et al. 13). Patients may respond in a variety of ways: crying, shaking, shutting down, denial, shock, or anticipatory grief. Closing the interview with further information regarding the diagnosis or treatment options provides hope and improves patient satisfaction (Prado et al. 16). As Prado et al. suggest, "Patients need time to adjust to the information given" (13). Supporting Buckman's suggestion of asking patients how much information is desirable, Prado et al. write, "studies around the world have pointed out that not all patients want to know their diagnosis and prognosis... doctors need to know and ask what the patient wants before giving information about the patients' health" (16). Communicating the correct amount of information for a given patient presents a communicative challenge for physicians; however, as Prado et al. assert:

If doctor[s] don't ask the patients what they want to know, not only unwanted information can be given, but patient[s] may try to find out about the disease outside the doctor's office like on the internet. Studies have shown that this type of information can be misleading and inappropriate which can lead to divergence in expectations between patient and doctor and ultimately a loss of trust. Studies also reported that patients want to understand risks and benefits of treatments like chemotherapy because not all of them want a prolongation of life in exchange of potential side effects. (17)

Prado et al.'s study supports that patients want family or friends present for the diagnosis, to receive as much clear and written information as requested, opportunities to ask questions and discuss their emotions, and to feel reassured (17). Additionally, patients "want their preferences 
to be respected by the clinicians" (Prado et al. 16). While this survey concludes that "good communication can decrease malpractice liability for physicians" (17), it also acknowledges that time constraints damage physicians' abilities to prepare for these difficult interviews, as well as diminishing patients' time to process the information (Prado et al. 17, 18).

Imaging has become an important tool for information gathering towards diagnostics; however, the implementation of this technology is far from perfect. Shawn Anthony et al.'s fouryear radiological study regarding the communication of critical imaging results indicated issues in interprofessional communication that can impact patient health. Suggesting that $10 \%$ of imaging results contain crucial information, it is imperative that such results be communicated to primary care physicians and teams in an urgent manner; however, this does not occur consistently. This study suggests that, "Failures in communication of abnormal imaging results are common," and also that, "Delays and failure in communicating critical results pose threats to patient safety and are a leading source of medical malpractice claims in radiology" (Anthony et al. 803). This 2011 study, based on recommendations presented by the Joint Commission, American College of Radiology, and Massachusetts Coalition for the Prevention of Medical Errors, championed the colour coding of imaging results dependant on urgency. Implementation of this policy resulted in improved timely communication of imaging results, and compliance to guidelines (Anthony et al. 806).

Emergency resident Damian Caraballo shares an incident indicating how deceiving imaging, in this case X-rays, can be in diagnostics. A dishevelled, overweight, middle-aged man with both gastrointestinal (GI) and psychiatric histories arrived in emergency in apparent and acute pain. Caraballo's immediate diagnosis was a perforation somewhere in the GI tract, a medical emergency known as Boerhaave's syndrome (208). The diagnostic tool commonly used 
to identify this syndrome is an X-ray showing air in the chest or abdominal cavity; however, the X-rays showed nothing (Caraballo 209). With the patient still in agony, Caraballo ordered additional tests — none of which show anything abnormal. He called on other professionals: his attending physician, the radiologist, the GI fellow he wakened with a phone call. With additional pain medication, the patient's writhing began to lessen. Caraballo ordered yet more tests, including another set of X-rays and a CT scan. The second set of X-rays revealed "free air" in the chest, supporting the original diagnosis; unfortunately, after three hours of listening to the patient's suffering, the patient's wife's nagging, and dealing with other emergency patients, Caraballo's judgement was also suffering, and he was now too awash in patient data to comprehend the finding's significance. Finally, the CT scan located a three-centimeter tear in the esophagus, the surgeon was called, and after eight hours in emergency, the patient was wheeled into surgery (Caraballo 209). Because of the diagnostic, and therefore treatment, delay the patient's recovery took forty-five days. Upon reflection, Caraballo recognized that his initial intuition was correct, but he had allowed himself to be swayed by others' professional opinions, the patient's psychiatric record, and because of his own lack of confidence, he just kept ordering more tests. Where did he get off track? When the first set of X-rays failed to show "free air" in the chest. The imaging caused him to dismiss this diagnosis, even though "free air" is not revealed on X-ray in $10 \%$ of patients in the early stages of Boerhaave's syndrome. Dismissing this diagnosis meant that he neglected to take more aggressive action towards this diagnosis, for instance by requesting a barium swallow or by consulting a surgeon earlier. The growing pile of unnecessary data, and the acute state of the patient, caused additional confusion that clouded Caraballo’s clinical judgement (210). 
The availability of online health information (of varying quality), and improved patient education, has led to emancipated patients who sometimes request unnecessary imaging, adding to physicians' challenges. Leah Rosenberg, of the Mount Sinai School of Medicine in New York, contends that, while patients should advocate for themselves, physicians should refuse unnecessary patient requests because they contravene professional boundaries, and can adversely impact the patient-practitioner relationship (22). Her suggestion for overcoming patient insistence for unwarranted imaging (e.g., full-body CT scans marketed directly to consumers), is to probe more deeply into the patient's concerns; in other words, communicate with patients to understand their motivations for the request. Taking a patient-centred approach, educating the patient, and rebuilding the patient's trust in the physician's clinical judgement, may help patients avoid unnecessary imaging.

Physician communication with older patients can present unique challenges, but physicians must also avoid making assumptions about their older patients' abilities. Sarah Barnes et al. researched communicative styles between primary care givers and older cardiac patients in a 2006 British study. Their key findings were: heart failure can be difficult for primary care physicians to diagnose; physicians avoid using the word "failure"; patients often have a poor understanding of heart failure, often due to receiving overly complex information; and little discussion of the prognosis for heart failure (Barnes et al. 488). This study underscores the complex problems of communicating with older patients, as acknowledged by both patients and physicians. Older patients can be complex patients, with multiple co-morbidities; physicians sometimes assume that such patients will die of something else before heart failure, so they feel it is unnecessary to discuss terminal heart failure (Barnes et al. 487). The rhetoric of "failure" (as in heart failure), was problematic for physicians, and was often avoided; consequently, patients 
were sometimes unaware that they were dying until they were admitted to emergency care (Barnes et al. 482, 485-486). "With improving skills and technology, modern medicine has moved towards the desire to fix diseases, with a tendency to keep implementing treatment regardless of outcome. Physicians often find it difficult to communicate a poor prognosis and the possibility of death is glossed over or avoided altogether" (Barnes et al. 488). Patients often found the medical language confusing and unhelpful, sometimes causing them to tune out (Barnes et al. 485). This demographic is less likely to ask questions, and more likely to defer to the doctor, even when this adds to patient frustration (Barnes et al. 488). The study concludes with a call for improved education and communication; however, in patients with potential memory loss or confusion, common with heart failure, this too is challenging (Barnes et al. 483). Finally, this study suggests that physicians, "tailor information to patient's individual needs" (Barnes et al. 488). In other words, physicians should be patient-centred in their communication to ensure that patients (and, with permission, their families) receive the information they wish to receive, in consideration of their ability to understand, the potential impact of this knowledge, and their desire to know.

Similarly, a 2006 study by Liang et al. regarding communication between older women and physicians concluded that, "physician communication styles characterized as deep, trusting, and bonding were associated with patient satisfaction" (390); these were often long-term professional relationships. The study's patients were women with an average age of 74.5 (Liang et al. 388). Although the encounters included some joint decision-making, once the discussion turned to mammography (the research focus), $60 \%$ of patients did not ask questions, and most physicians did not offer additional information (Liang et al. 389). Physicians discussed and informed patients on topics raised by patients over $90 \%$ of the time; however, they only elicited 
areas of discussion from patients $18 \%$ of the time, and treatment plans were rarely negotiated ( $9 \%$ of the time), leading to the conclusion that although physicians were skilled at initiating communication, they were less able to elicit patient concerns or negotiate treatment options (Liang et al. 389-390). Again, this study speaks to physicians holding power in the clinical interview.

A 2013 Agenda panel titled "More or Less Medicine" on TVO explored the changing complexion of healthcare in Ontario. During the panel, Dr. Danielle Martin, a family practitioner and Vice President of Medical Affairs and Health System Solutions at Women's College Hospital, suggested that fewer checklists and more talk would benefit patients. She also acknowledged that,

The complaint that you tend to hear from doctors is not so much that they don't want to listen to their patients, it's that they feel caught on a treadmill that they can't get off of and that the pressures of practice with a line-up of patients that they can't seem to get on top of, and the mounds of paperwork that they can't seem to get to the bottom of, make it really difficult to give people the time and attention that they wish they could, and so I don't think it's a question of having to retrain (Martin quoted on More or Less Medicine).

Dr. Martin also suggests, "perhaps we need to spend a little bit less time on the technology aspect of things and a little bit more time looking at the whole human being sitting in front of us" (quoted on More or Less Medicine). Technologies have enticed doctors way from listening, a skill that is foundational to good patient care.

This same panel featured Dr. Doug Weir of the Ontario Medical Association, who asserted that a serious issue with instrumental medicine is that it can create, "false reassurance... 
that if it's all clear, that somehow you're in good health," when this may not be the case, and may not be reflective of the patient's experience (quoted on More or Less Medicine). Sholom Glouberman, President of Patients Canada, adds, "The idea of going from an instrumental kind of medicine to a more human and individualized kind of medicine really does come from that shift in morbidity. ... People aren't benefitting from old-style instrumental medicine and they need now a kind of medicine that is individualized and relationship based" (quoted on More or Less Medicine). Glouberman suggests that, due to advances in medical science, populations are moving away from acute or infectious health concerns, and towards long-term chronic manageable health concerns. Chronic disease management, for condition like diabetes or mental health, requires knowledge about diet, activity, and personal habits only available through conversation.

Because the nature of people' illness and people's health has changed, we think that patients and their families have to be far more involved in the healthcare system....They have to be engaged in policy development, and in thinking about these policies together with the providers. They have to be part of groups that redesign services.... There are lots of things where patients' perspective and patients' view of these things would really make a difference both in regularizing and in getting better relationship with doctors and patients (Glouberman quoted on More or Less Medicine).

Along with improved patient education, patients must learn to advocate for themselves in a changing healthcare environment. For older patients, whose ideas of the patientphysician relationship evolved when physicians held an almost god-like position, this sort of shift may be very difficult. 


\section{A Multivalenced Methodology}

I'm interested in the murky areas where there are no clear answers-or sometimes multiple answers. It's here that I try to imagine patterns or codes to make sense of the unknowns that keep us up at night. I'm also interested in the invisible space between people in communication; the space guided by translation and misinterpretation. This space highlights the inevitability of solitude and the impossibility of true understanding.

\section{Taryn Simon (quoted in Cornell)}

John Creswell defines ethnography as "a way of studying a culture-sharing group," adding that it offers a way of "Describing and interpreting the shared patterns of culture of a group" (68). In this dissertation, physicians and patients can be seen as sharing the culture of the clinic. Ethnographic research generally involves interviews and observation of a group, decocted into a written descriptive analysis (Creswell 79). While Creswell outlines the broad strokes, this dissertation seeks something deeper, a type of "thick description" (Geertz 6). To gain this deeper interpretation, this dissertation draws on Hubert Knoblauch's (2005) concept of focussed ethnography. Knoblauch indicates that focussed ethnography is most appropriate for research within complex societies, such as the clinic, around questions of communication and interaction. As it has been used for medical research in nursing, and workplace studies in high-tech environments, it seems a natural fit for this dissertation. While similar to traditional ethnography, focussed ethnography has some marked differences that allow for a deeper, more detailed analysis of the group and its activities. Whereas traditional ethnographies rely on long-term field work, providing an intensity of experience, typically recorded in note form, focussed ethnography relies on short-term field visits, with a focus towards the data and analysis, over a 
shorter period of time, and electronically facilitated data collection that frees the researcher to critically observe and reflect as encounters are occurring. Because focussed ethnography tends to produce a large amount of data over a relatively short time, greater emphasis is placed on data analysis: How does the communication take place? How is the technology used in practice? This researcher's experience with over a decade of professional standardized patient and patient simulation work also provides background knowledge Knoblauch cites as necessary for undertaking focussed ethnographic research. While ethnography provided the theoretical boundaries for this research, data analysis was undertaken using a hybrid combination of qualitative discourse analysis strategies, as outlined later in this section.

Studying patient-practitioner communication in the presence of biomedical technologies required interviews, and the observation of patient-physician encounters. The most obvious way to gather data would be in a hospital, but the formal hospital research ethics process was daunting and would entail a lengthy waiting period, with no guarantee of success. ${ }^{74}$ Additionally, in-situ hospital research is tricky; what is designed as a purely observational study may be difficult to maintain if patients or families commandeer the researcher as an advocate or liaison with medical personnel (Catherine Schryer, personal communication). The most obvious approach might not be the most feasible, and so it was necessary to develop a different mechanism for gathering data.

After receiving Ryerson Ethics Board approval for the field research, the first phase was to conduct semi-structured interviews to access older patients' experiences in the presence of physicians and medical imaging technologies, such as ultrasounds, MRIs, CTs, and angiograms.

\footnotetext{
${ }^{74}$ I also had personal ethical concerns about requesting consent for either interviews or observation of patientphysician encounters when a patient might be receiving life-altering news. Additionally, because of personal experiences advocating for elderly parents in hospitals, I was uneasy about entering this context as a researcher. Researchers are never wholly objective, and in this case, my personal stake in the work seemed likely to compromise the research if I embedded myself in this environment.
} 
Based on Canadian and international definitions, the minimum age for interview participants was set at sixty-five (Service Canada; World Health Organization). Older interview participants, aged sixty-nine to eighty-six, with appropriate experience, were recruited using convenience and snowball sampling. As this researcher is not qualified to assess mental status, the assumption was made that individuals living independently were compos mentis and therefore able to give the required consent to be interviewed. The interview questions focussed on communication before, during, and after imaging procedures (see Appendix C). The aim of these questions was to facilitate the participants' telling of their stories, accessing the patient narratives, and providing a wealth of information. Seven interviews were undertaken, varying in length from forty-five minutes to two-and-a-quarter hours. Participants described their experiences, as well as reflecting on the impact of imaging sessions, and their communication with physicians and other healthcare practitioners. The interviews were analyzed using a hybrid discourse analysis, as detailed below. Analysis of the interviews is offered in Chapters 4, 5, and 6.

Epstein et al. acknowledge the "methodological problems in collecting data from interactions between physicians and actual patients," including issues of consent, behaviour modification due to observation, and the limitations of observing relatively few encounters (1519). To avoid these and other problems, the second phase of research, the patient-physician encounters, was undertaken using simulated patients (SPs). As defined by Dr. Barbara Stubbs, one of the founders and the original director of the SP program at the University of Toronto, SPs are healthy people who have been, "trained to portray the historical, physical and emotional features of an actual patient." This researcher has many years' experience with SPs and, having worked in medical education at the University of Toronto's Standardized Patient Program, is very familiar with the value of SPs in medical communication training, examination, and 
research with both students and practicing professionals. The use of SPs avoids repeatedly subjecting patients to situations that might be physically or emotionally stressful, as well as ensuring that each medical participant receives a similar patient experience.

Using the phase one interviews to access patient language and experience lent credibility to simulated roles developed for this research (see Appendix D). Based on their prevalence in the population, the roles focussed on heart disease and cancer, with one benign role and one role with findings for each disease (four roles in total). Each role was supported and informed by medical images from educational sources, with accompanying radiologists' reports. For example, an initial interview regarding an angiogram experience offered material for a simulated heart disease role, supported by angiogram images, and the accompanying radiologist's report. Each scenario was encountered by physicians, both generalists and specialists, who were asked to explain the imaging results and diagnosis, as well as discuss treatment options, with the SP. This series of encounters between physicians and SPs, in the presence of diagnostic images, added to this study's exploration of patient-physician communicative techniques with accompanying images. Epstein et al. suggest that, "Standardized patient methods can assess the behaviors of many physicians ... across a wide variety of medical conditions and psychosocial profiles..." The one drawback Epstein et al. suggest is that because SP visits are usually initial visits, they may fail to fully capture a physician's normal communicative style (1519). SP-physician encounters were observed, and also video recorded, for further analysis (see Appendix E).

In some situations, notably assessment, SPs are instructed to adhere strictly to a standardized delivery of the role, sometimes verbatim, regardless of the dynamics of a given interaction; in others, they are instructed to respond to the physician's mannerism and words more freely. In this research, although the SPs were trained on specific roles, they were free to 
respond to a given situation in the moment; in other words, if a physician said or did something that evoked anger or fear, the SP would respond honestly out of anger or fear. SPs are useful for educational and research purposes, highlighting that these are encounters between two individuals, a professional and a lay person, and helping to focus on the communicative dynamics. SP training consisted of individual training sessions with the researcher to read and discuss the role, as well as review of video links clarifying the patient's imaging experience back story.

Senior SPs are trained to analyze the communication in each encounter and provide articulate and insightful feedback, enriching post-encounter data collection. After each encounter, the researcher undertook one-on-one semi-structured interviews with the physician and the SP (see Appendix F). These questions helped focus the physician or SP on the effectiveness of both verbal and non-verbal communication techniques deployed during the encounters, as well as the effect of having medical images present.

Although, given personal experience, it was natural for this researcher to turn to SPs to help resolve issues of in situ research, most SP roles are developed by medical professionals drawing on their own clinical knowledge and experience, rather than medically untrained humanities graduate students. The researcher's medical knowledge, although above average for the general public, and further informed by recent imaging experiences, was tested in the creation of believable SP roles, and this arguably led to some problems with one role, as discussed in the following findings.

As is apparent from this chapter's review of literature, much of the research into patientphysician communication has been undertaken by medical researchers, usually deploying quantitative analysis techniques. This researcher felt it was important to move beyond statistical 
analyses and focus on qualitative methods of data analysis. Grounded theory was briefly

considered, using NVivo or similar software; however, the researcher felt that the resultant

analysis leaned towards the quantitative, even as it strove to be qualitative. The next pull was

towards both Arthur Frank and Michel Foucault, indicating some hybrid form of qualitative

discourse analysis to interrogate the raw data in audio, audio-visual, and verbatim text ${ }^{75}$ formats. $^{\text {. }}$

The first phase of field research draws on Arthur Frank's notion of patient narrative, as an

attempt by patients to make sense of and communicate their experiences of illness, often using

metaphoric language. ${ }^{76}$ Letting Stories Breathe: A Socio-Narratology was published in 2010,

marking a practical advance in Frank's thinking about narrative performance, structure, and

\footnotetext{
${ }^{75}$ Transcription is labour-intensive and, due to concerns that undertaking this aspect of the work would retard my progress, I contracted a trusted and capable colleague to undertake this work. He also willingly signed a confidentiality agreement.

${ }^{76}$ First presented in 1995 in The Wounded Storyteller: Body, Illness, and Ethics, the revised 2013 edition adds considerably to the book's argument. Frank contends that patient narratives can be categorized as archetypal, "restitution, chaos, and quest" stories (WS xiv), each having unique characteristics. The restitution narrative is the story in which the patient is well, becomes ill and returns to wellness; the more complex chaos narrative is an "antinarrative" descending into fear and anxiety, lacking rational control (Frank, WS 98); and the quest narrative is one in which the journey back to health is accomplished with mythic heroic proportions, underscored, as Anne Hunsaker Hawkins notes, by elements of rebirth or renewal after both a physical and metaphysical journey (31). Since 1995, these three patient narrative types have been used countless times by other researchers, as if they were the only three narrative types. In the Afterword of the revised edition, Frank reiterates that, "other types can and should be proposed," pointing to the "political/environmental narrative" he had also briefly suggested in the original edition (WS 76; WS 197n2). In the revised edition's Afterword, Frank develops three additional narrative types: "life-asnormal narratives, borrowed stories, and broken narratives" (WS 193). Briefly, the life-as normal narrative is a nonnarrative that minimizes or denies illness, and the patient who insists that everything is fine, when it is not (Frank, WS 193-197); a borrowed story is similar to a quest narrative, but is co-constructed based on an existing narrative (Frank's example is a child-patient who becomes fictional character Buzz Lightyear; WS 197-201); broken narratives are those of patients incapable of telling their stories, whether due to speech, mental, or memory problems (WS 201-204). Although these narrative categories are not used in the present research, they are a keystone of patient narrative research worth acknowledging. Frank's more recent notion of "letting stories breathe" is more actively used in this dissertation.

To Frank's expanded list, I would like to add the possibility of an acquiescence narrative. I suggest that the acquiescence narrative is the story of the patient who has accepted his or her mortality, accepted that he or she will not recover from illness, and that death is both inevitable and close. This is the narrative adopted by some elderly patients, as well as some patients suffering from chronic long-term disease. The acquiescence narrative may or may not be outwardly rational, or it may contain rational but decontextualized elements; for instance, the patient may be lucid, but believe that he or she is in a different place. The acquiescence narrative voices a personal experience of illness that may be less dominated by preconceived notions of consensus reality. The acquiescence narrative is incommensurable with techno-medical discourse, may be perceived as irrational, and may be explained in biological terms of brain oxygen deprivation. The acquiescence narrative is that of the patient at peace with his or her imminent death. The supposition of an acquiesence narrative is based on informal research, but may be a site for future research.
} 
analysis. Focussing on the notion of "dialogical narrative analysis," this text offers a loose method for discovering the effect of a story, rather than just its content (Frank Letting Stories Breathe, 71). Emphasizing dialogue's natural flow and flux, Frank considers dialogical narrative analysis a form of criticism, rather than a method, a temporary tool for interaction ( $L S B$ 73). In Frank's words, “dialogical narrative analysis refuses to say, as too many methods do effectively say: these are the rules; here are the steps to implement these rules" (LSB 73). Frank suggests analyzing stories using the following questions:

"What does the story make narratable?" (LSB 75)

$>$ "Who is holding their own in the story, but also, is the story making it more difficult for other people to hold their own?" (LSB 77)

$>$ "What is the effect of people being caught up in their own stories while living with people caught up in other stories?” ( $L S B$ 78)

"What is the force of fear in the story, and what animates desire?" ( $L S B$ 81)

$>$ "How does a story help people, individually and collectively, to remember who they are? How does a story do the work of memory?" (LSB 82)

Frank stresses that this is not a definitive list, and also that, depending on the story, some questions might be more or less relevant.

Frank also emphasizes that "interpretation is always a work in progress" and that dialogical narrative analysis necessarily has "considerable ambivalence toward interpretation" ( $L S B$ 87). The reason for this ambivalence is that interpretation often marks an end-point, a finalization, a declaration of the truth, closing further analytical opportunities; at this point, stories no longer breathe, but suffocate ( $L S B$ 87-88). The potential multiple truths of a story can only be discovered by letting it breath, keeping it alive, through multiple interpretations ( $L S B$ 
91). In this context, "Interpretation becomes a decoding scheme: the capacity to sort what makes a difference from what is secondary or contingent, to trace the cause of what matters, and to name that cause" (LSB 93).

Dialogical narrative analysis also adjusts the location of the researcher, and the relationship between the researcher and her participant. The researcher acknowledges that she has her own stories, some of which may overlap with the storyteller's, but also that the story may require the researcher to stretch into the unfamiliar ( $L S B$ 96). Rather than using the terminology of "research subject," Frank prefers a shift to "research participant," acknowledging that, "Participants are experts, at least in their own lives, and the dialogical interviewer is there to learn from the participant" (LSB 98-99).

To undertake dialogical narrative analysis, "the analyst's work and practice lead to hearing multiple stories about similar events or experiences. Analysis can connect these stories" (LSB 102). This type of analysis "is less a matter of decoding stories than of seeing all the variations and possibilities inherent in the story...Interpretation aspires to be an ongoing dialogue with the story" (Frank LSB 104). Frank also aserts that this type of "interpretation can only proceed slowly" (LSB 108), allowing it to dovetail nicely with Andrea Doucet's method of "slow scholarship," discussed below.

The search for a Foucauldian discourse analysis was wandering, as Foucault himself did not develop a methodology for discourse analysis. Various conceptions of Foucauldian discourse analysis have developed, rooted in his work, and somewhat overlapping, some more structured than others, and emphasizing different Foucauldian texts. Jean Carabine's case study of Foucauldian discourse analysis asserts that "there are 'no hard and fast' rules which set out, step by step, what a genealogical analysis is" (268). Carabine suggests that 
“discourse/power/knowledge are an interconnected triad," coming together in Foucault's notion of geneology (267). The patient-physician interaction is clearly a site of discourse, begging questions regarding power and knowledge. As Carabine sees it, "Foucault sought to trace the development of knowledges and their power/knowledge in modern society. Genealogy is concerned to map those strategies, relations and practices of power in which knowledges are embedded and connected" (276). The clinic is a complex discursive site in which knowledge is coproduced by patient and physician; assumptions are often made about who holds the knowledge and power in the clinic, but are these assumptions necessarily always true?

Foucault's complex power/knowledge concept seems inherent in the patient-physician relationship. Marianne Jørgensen and Louise Phillips assert that power, as a productive rather than oppressive force, is necessily bound up with knowledge and with discourse (13-14). They also assert that much of what claims to be Foucauldian discourse analysis is "relatively rulebound sets of statement which impose limits on what gives meaning" (Jørgensen and Phillips 13). Conversely, Jørgensen and Phillips suggest that, "Truth is a discursive construction and different regimes of knowledge determine what is true and false" (13). This flexibility of truth, dependent on the players and context, was important to ensure fair analysis of this research. "Because truth is unattainable, it is fruitless to ask whether something is true or false. Instead, the focus should be on how effects of truth are created in discourses. What is to be analysed are the discursive processes through which discourses are constructed in ways that give the impression that they represent true or false pictures of reality" (14). Subjective truths are slippery; this does not, however, invalidate them. Echoing Frank (and Thomas King ${ }^{77}$ ), Jørgensen and Phillips assert that "subjects are created in discourses" (14).

77 "The truth about stories is that that's all we are" (King 2). 
While attempting to comprehend this swirling array of ideas, the researcher attended a Qualitative Analysis Conference ${ }^{78}$, and was introduced to Andrea Doucet. Doucet, and co-author Natash Mauthner, suggest that a "narrated subject" can circumvent problems of a constructed subject in research (399). Access to the "narrated subject" is achieved through "the Listening Guide," an approach developed at Harvard by Lyn Mikel Brown and Carol Gilligan. Again, the focus is on "research subjects' stories" rather than imposing a research frame onto the narrative. This narrated subject is "constantly changing," and rather than being a defined subject, is a relational subject, that always, necessarily, retains hidden or unknowable elements (Doucet and Mauthner 402-404).

Brown and Gilligan developed "the Listening Guide" in their research for Raising their Voices: The Politics of Girls' Anger (1999), to help them understand and interpret interview data. Fundamental to their research were the questions: "Who is speaking and in what circumstances" and "Who is listening and what is her relationship with the speaker-especially with respect to power?" (32) They suggest that the Listening Guide is, "sensitive to the polyphonic nature of voice, the nonlinear, nontransparent interplay and orchestration of feelings and thoughts, as well as to the issue of power..." (Brown and Gilligan 32). Similar to Frank's notion of letting stories breathe, the Listening Guide offers, "an interpretive, dialogical approach," and it is maleable for deep analysis of different types of research questions (Brown and Gilligan 32). This style of analysis requires multiple readings or listenings to the data, each time focussing on a different element. For their research, Brown and Gilligan reviewed each narrative four times, asking 1) what shape does the narrative take and how does it relate to the researcher?, 2) Where is the first person voice and how does it relate to others?, 3) Where is a sense of personal anger and social

\footnotetext{
${ }^{78}$ The $29^{\text {th }}$ Annual Qualitative Analysis Conference: Cultures of Narrative/Narratives of Culture took place June 2022, 2012 at Memorial University in St. John's, Newfoundland.
} 
critque in the narrative?, and 4) What constructed or personal notions of feminity appear in the narrative? (33-34).

Doucet and Mauthner have adapted the Listening Guide for their own research and methodological considerations. This researcher had hoped that their volume, Slow Method: ReMaking the Listening Guide, would be published in time to assist with this dissertation; however, perhaps appropriately, they are still writing this volume and Sage has twice delayed publication. In their 2008 methodological article, the authors also suggest four readings, each with a unique focus: 1) "Relational and reflexively constituted narratives" to map common themes or words, and the researcher's reactions, 2) "Tracing narrated subjects" to focus on the storyteller's sense of self and self in the world, 3) "Reading for relational narrated subjects" to examine the storyteller's relationships and social networks, and 4) "Reading for structured subjects" to examine "structured power relations and dominant ideologies that frame narratives" (Doucet and Mauthner 405-406). This final component pulls the methodology in a Foucauldian direction.

Doucet's keynote address focussed on the related concepts of "slow scholarship" and "The Listening Guide," as well as notions of reflexivity and the place of the researcher in the research. The notion of reflexivity made this researcher more conscious of her place in the work, and as a field researcher. As Doucet said, a scholar is a sort of cab driver; the discussion of the sights along the route may alter the route itself. Her notion is that all our research reveals is stories; we can never know the research participant. Stories provide the opportunity for the researcher to explore the space between herself and the research participant. "There is no data; only your relationship to the data, and everyone's relationship is different." Ultimately, according to Doucet, there is no participant and no data: there is only a narrative process. All one 
can know is one's relationship to the story. Doucet seems to connect strongly to Frank (and to King).

Taking cues from Frank to let stories breathe, and from Doucet to carefully re-read stories multiple times, the following questions evolved from the data gathered for this dissertation:

What emotions are described?

What physical sensations are described?

What communicative experiences and rationalizations emerge about imaging or the medical context?

$>$ Does the patient respond to notions of power or control? How?

These are the questions used to interogate the patient interviews in the following three chapters, while the Observation Protocol developed for this reserach was used to examine the simluated patient-physician encounters discussed in Chapters 7 and 8 (Appendix E). By delving into these stories, this researcher hopes to begin to answer the primary questions guiding this dissertation:

How does the tension between the patient-centred approach and advanced biomedical imaging technologies affect patient-physician communication, especially where older patients are concerned?

$>$ Is it possible to retain a patient-centred approach to healthcare while optimizing practitioner use of biomedical technologies?

\section{Conclusion}

This chapter completes the review of literature by focussing on the challenges of patientphysician communication, the use of imaging in clinical encounters, and the responses of older patients in clinical encounters. It then reveals the methodological strategies used in this 
dissertation, beginning by discussing ethnography, gathering patient interviews, developing roles for and using simulated patients, and ending with a discussion of the development of an analytical strategy drawing on both Frank and Doucet, against a Foucauldian background. The following chapter offers an analysis of the older patent interviews focussing on the first two questions: What emotions are described? and What physical sensations are described? 


\section{Chapter 4: Older Patients' Imaging Experiences}

Particularly critical has been withdrawal of attention from personal, cultural, and social expressions of illness by technologies designed to explore or treat its physical aspects.... Humanistically, the subject of care is the person, who cannot be understood fully if divided into parts by the thinking and technology of medicine.

Dr. Stanley Joel Reiser (TM 188)

Physicians are often more comfortable with the medical discourse of disease than with the emotional, convoluted, metaphoric language of the patient's illness experience. According to Arthur Frank, illness narratives are the patient's attempt to re-orient the body, to restore order, but also an acknowledgement of the disrupted rhythm of life through illness; stories provide a way of integrating the illness experience into one's life (WS 2). Evidence exists, however, that seemingly objective medical tests and images can take precedence over the patient's history, narrative, and subjectivity (see, for example, Blaxter CVP 769).

Published narratives of the imaging experience clearly show how difficult it is to verbalize the effect of imaging on patients. Scholars experienced with using words in complex circumstances struggle with the language to effectively communicate such experiences. Sociologist Regula Valérie Burri notes, "few studies...have looked at how the body is involved in the very process of medical image production and at how it interacts with machines, instruments, spatial arrangements..." (109). In part, this dearth of research may be because, as a communicative and experiential territory, imaging is so complex and so difficult to express cogently. A review of the limited literature in the area of the patient experience of medical imaging reveals the ways in which researchers have struggled for expression. The images 
themselves are alluded to as mirrors (Radstake 116; Wall 139); evocative of a sense of ownership and bodily responsibility (Blaxter CVP 771); something that "provokes simultaneously a curious sense of detachment from my own anatomy and a feeling of inhabiting, or being inhabited by, the image" (Wall 142); perhaps reflecting the complex notion of "distributed embodiment" in which "bodies are multiplied to include images," simultaneously both here and there (Radstake 129, 134). Images create a strained mediation in our day-to-day entwined subject-object sense of embodiment (Radstake 34). Medical illustrator Shelly Wall suggests that "self-objectification" through the imaging experience leads to feelings of vulnerability and violation, and that, "Graphic representations of the body seem to both construct and trouble a person's sense of embodiment. They provide cognitive information that, like illfitting clothing, sits uncomfortably with how a person inhabits her body and alters how she moves and feels in it" (139-140). The othering effect of the image outside our lived-in bodies creates a unique self-consciousness.

What are the implications for older patients confronted by high-tech images of their bodily interiors? To begin to answer this question, seven older patients over age sixty-five (aged sixty-nine to eighty-six), with various medical imaging experiences, were interviewed. Semistructured interviews supported the sharing of patient narratives, focusing on emotional and communicative aspects of their experiences before, during, and after imaging appointments. As detailed in the previous chapter, this data is interrogated qualitatively using an adaptation of Andrea Doucet and Natasha Mauthner's “Listening Guide,” which supports a form of Foucauldian discourse analysis, coupled with Frank's notion of "letting stories breathe" (Doucet and Mauthner 405-407; LSB 4). Based on the research questions, the interview questions, and 
after reviewing the interviews, the following four questions are used to explore the interview data:

What emotions are described?

What physical sensations are described?

What communicative experiences and rationalizations emerge about imaging or the medical context?

$>$ Does the patient respond to notions of power or control? How?

For each question, each transcript and audio file is reviewed in detail, so each interview is reviewed multiple times. While each question is asked separately, there is overlap between these categories. Analysis was further complicated by the medical imaging context; images are taken to diagnose illness, so patients also encounter physicians and other medical personnel. Increasingly, hospitals employ a team approach to patient care; while acknowledging this, discussion of the team and encounter with its members would have expanded this dissertation to an unwieldy scope, so the focus remained on the physician. Additionally, diagnosis leads to treatment or surgery, so the interviewed patients often shared their larger medical experiences, some of which spoke to the posed questions. The depth of analysis, supported by direct quotations from the interview participants, yielded a chapter of unwieldy length, so it has been divided into three. This chapter analyzes patients' internal experiences, their emotions and physical sensations; the analysis of relational experiences - their communicative experiences and rationalizations, and the Foucauldian discourse analysis of their responses to power or control— comprise the following two chapters. All three chapters qualitatively analyze the seven interviews, exploring the ways in which these patients' experiences echo, or diverge from, each other, or published patient accounts. 


\section{What emotions are described?}

I don't mind going on rides at Canada's Wonderland, or the CNE, or any of those things, so I don't mind things that sort of take you to the brink of wondering, am I going to have a heart attack with this whole thing or what? I quite enjoy it. But that [MRI experience] doesn't sort of rate up with rides at the CNE or wherever.

\section{Patient D}

Patient A also used the metaphor of "a rollercoaster ride," adding that the medical experience creates "the gamut of emotions..." The emotions emerging from the data are complex; while the expressions of their experiences often agree, patient experiences sometimes conflict with each other, and sometimes the same patient contradicts himself or herself. When specifically asked how she felt during her imaging experiences, Patient $\mathrm{C}$ initially responded: "You know this is the kind of question that your generation asks. How did you feel? I don't know how I felt. Somebody told me that I had to have the thing. You go and you have it and you don't.... I had no feelings.” Although this reply suggests a neutral emotional response to the imaging experience, several emotions did emerge through her and other patient interviews. Notable too is the obvious Foucauldian undertone of an unquestioning, disempowered patient doing what she is told; she could have refused imaging.

The most commonly mentioned emotion was fear, although it is often difficult to determine whether this is fear of the imaging session, fear of a potential disease state, or a combination. For instance, when Patient B responds, “obviously emotionally it's threatening because of the risks of a brain angiogram," this seems a clear response to an invasive imaging procedure; however, underlying that apparent fear is the fear of illness, incapacity, or potential death. Separating the fear response into different causes is challenging. In Patient G's words, 
“...you know we're all scared when you're going out for these things. You're terrified. You don't know what's going on with your body—and usually it's nothing. But it's scary. You just don't know." Later in the interview, she repeated this theme: "You're scared. 'Oh is it cancer? Is it an aneurysm?' Because you know something's wrong, but you don't know the seriousness of it. And so the waiting is so long. It just — and then you're scared to death when you go the doctor because you've waited this long." This statement confirms that patients do begin to wonder, to guess at what may be wrong, especially when a long wait is required for imaging results.

Although Patient G has never had one, she declares, "I'm frightened of an MRI." This fear is based on things she has heard from other patients and her sense of claustrophobia. Similarly, Patient A says, "I've never had an MRI. I would find that difficult. But, even with a CAT scan I think it's important to have someone in the same room that you can see, and they can see you; that sense of someone who is here, so you're not alone. ...but certainly a good claustrophobia comes into play with machines that you go into." Although they have no direct experience, these patients express fear of certain types of imaging, based on discussions with other patients, and their imagining of the experience inside an MRI.

While Patient $\mathrm{G}$ initially states that she was not frightened during a recent bone scan, ${ }^{79}$ she later reflects that this scan was, "very, very slow and they do the whole—like they did from head to toe. And it's really, really, really slow. It felt like it was forever when it was here [indicating thoracic area]. But they were so close, so close. And I thought, "What if that thing fell. It would completely crush me."” As this and other interviews indicate, even when patients

\footnotetext{
${ }^{79}$ From the interview, it was unclear whether Patient G had a bone density test or a bone scan. A bone density test is a series of low radiation X-rays for detecting osteoporosis. A bone scan involves injecting a radioactive tracer into the body, followed by a two-to-four hour wait as the tracer circulates; Patient $\mathrm{G}$ did not mention this step. Similar to CT or MRI scanners, the patient lies on a gantry as the scanner takes multiple images. Bone scans are used to detect several conditions, including metastasized cancer (Mayo Clinic). Given the patient's description of the scanner, and her medical history, it seems likely that this was indeed a bone scan.
} 
initially respond that everything was fine during their imaging experience, upon reflection, they often reveal that there was some fear that they had rationalized, perhaps to calm themselves during the procedure. Patient G's fear regarding MRI machines is perhaps well founded.

Commenting on his MRI experience, Patient D says, "Actually I found it very claustrophobic. I did not like that part of the experience at all. My feeling was if I wanted to get out, how could I get out? And I've never had that feeling before, so it was frightening. I'm sure they probably told me there was a quick escape route and all the rest. I don't know what he said, but I did not hear that." Patient D acknowledges that he felt "anxiety" about the possible diagnosis of a lump in his neck; adding to this anxiety was a claustrophobia-inducing imaging procedure. As previously indicated by Patient G, patients may already be thinking the worst when they undergo imaging procedures. Patient D reinforces this idea, commenting: “...it's all tied in together that you're there for a reason and there's a reason of suspecting; that they want to at least look further and that probably raises that whole level of anxiety. Anxious about the equipment, but also anxious about why would they even suggest that I come have a look." The patient's dread of bad news may create a strong negative emotional response, although nothing serious was diagnosed.

In a study regarding the effect of bad news on patient-practitioner communication, Ariadne Juna Fernandes do Prado et al. find that patients "hear but don't comprehend everything” (16). Or as Patient E reflects, “...sometimes, though I'm listening, I may not be hearing what they're saying.” Although Patient D may have been told about an emergency call button to press if he became panicked, he was unable to retain this information due to fear caused by a combination of an undiagnosed lump in his neck and his MRI machine encounter. As he expresses his claustrophobia, “you go head first, and it just seemed like a tunnel that wouldn’t 
end." Although other patients report that MRIs are extremely loud machines, when asked about the volume during his experience, Patient D reports: "That part of it I honestly don't recall. I think I was more sort of fixed on being in this tube and being extremely claustrophobic." Given that MRIs produce noise levels of 82.5 to 118.4 decibels, actually increasing when a subject is inside the machine (typical conversation is about 60 decibels and sounds above 90 decibels are considered damaging), Patient D's obliviousness to the noise is a comment on his heightened anxiety during the procedure (Price et al. 288, 291).

In spite of his extreme anxiety, Patient D expresses a need to be “macho." Patient D's masculinity may have prevented him from asking for any kind of sedation prior to the imaging procedure, although it might have been helpful in his case. Patient E also comments on the "macho" behaviour of male patients in his waiting-room observations, noting that in his estimation, men are especially uncomfortable with the feelings of vulnerability induced by illness. In a similar vein, Patient F comments, “...you can't say to the nurse, 'You know, I'm scared out of my mind.' Or you can't because we don't do that sort of thing."

Patient E provides a lengthy description of an imaging-related incident that he found "scary." Difficulties injecting the contrast medium for a CT scan undermined his confidence in the technicians performing the scan. In his words:

I don't like needles. I don't like those kinds of things. And I didn't know that that was going to have to be a part of it, so that was scary because it was a surprise. The person who gave me the injection for contrast, she was, I believe, a student, who had trouble perhaps asking for help. She had her supervisor in the room and the student who was giving me the injection was really not good at it. It was a bloody mess. 
And the supervisor, or her teacher, was in the room, and he knew she was making a mess of my arm, and seemed to think it was okay for her to keep practising. And she did. And it was-it truly was a bloody mess where it didn't need to be. So, I think for that I-I mean eventually I think he did, the supervisor did take over and found an intravenous, got me to the point where he could get me on the table. That was very scary, and it made me lose confidence in the technicians. And I'm someone who thinks that students should learn in a health-care setting. But I certainly at that point was, in a minor way, a casualty of one. It didn't work well for me. And I don't think the student felt very good about it either... So that was my introduction, and then again in the context of having a new diagnosis where this is not the treatment, this is the prelude to the treatment. So that was scary.

Coming back to this incident later in the interview, Patient E says, "Well, it's important for the student to learn, but don't make it too hard for the patient. Like, if you're not getting it after a couple times, say you don't know. Get some help and let somebody who can actually help the patient better at that point do it." Although he is supportive of medical students learning on patients, Patient E's unfortunate experience points to the need for limits to this kind of experiential learning.

Subsequent to his throat cancer diagnosis, Patient E underwent over a month of radiation and chemotherapy treatments. To ensure that the radiation was appropriately targeted, a mask was made for locking him to the gantry in a precise treatment position. The location of the 
cancer, and the treatment itself, caused nausea and difficulty swallowing; consequently, he was on a feeding tube for five months. Additional imaging was used to assess the treatment's success. When asked about the follow-up imaging, Patient E responds:

I was dreading and looking forward to both the MRI and the CATscans, the most recent ones. The dread is, I'm coming back in the patient role in an environment that really hurt me a lot, and can I—if it's not good news, can I tolerate more of that? Do I have the capacity to hear 'you're going to need more or different treatment?' And I'm not sure I have an answer to that question, but I'm pretty sure I'm in a different space this year this month, than I was last year this month.

Fortunately, in his case, the treatment eradicated the cancer. At the time of this interview, no further treatment is deemed necessary. In this case, Patient E experiences an anticipatory "dread" because of a combination of a familiar environment and an almost intolerable anxiety about the possibility that further treatment might be recommended upon review of the imaging results.

While the treatment that Patient $\mathrm{F}$ underwent, a quintuple heart bypass, was clearly explained (verbally and in print) and smoothly orchestrated, the same cannot be said of his cardiac angiogram. Of the patients interviewed for this study, Patient $\mathrm{F}$ is the most traumatized by an imaging procedure. To begin with, "I was not well prepared... I had no idea what was going to happen...." The lack of communication about the imaging procedure, and what he might expect, made something that was clearly routine for the medical staff into a highly stressful experience for this patient. Furthermore, the perceived lack of information provided to the patient may also constitute a breach of medical ethics. Although he will have given consent, his statement suggests that his informed consent was not given, or he would have known what to 
expect. When asked how he felt during the angiogram, Patient F responds: "Absolutely

frightened out of my mind.” The procedure was explained step-by-step as the team proceeded:

"When I went in, I was told they were going to make an incision in my left leg, and the tube would go up through into my heart, and they would be able to see it on the monitor"; however, this information came too late. By the time Patient $\mathrm{F}$ arrived in the imaging suite, he was already terrified. He requested, and was given, "something" to calm him down; "I must admit whatever they gave me did calm me to the point where I was able to-I just lay there and let things happen... They did not put me out. I was conscious through the whole thing, but I did not suffer any pain..." The fear, however, did not abate: "significant I think is the fact that I kept my eyes closed the whole time...I wouldn't open my eyes. I never opened my eyes from the moment I went into the room till the moment I came out." While he admits that the angiogram was fairly painless, psycho-emotionally it was clearly traumatic; since this incident, Patient F consciously avoids this hospital floor.

Another commonly expressed emotion is varying degrees of frustration. ${ }^{80}$ Patient $\mathrm{E}$ seems somewhat frustrated by an MRI experience because of the demands of the machine. Due to the location of his cancer, during imaging he had strict instructions regarding when he could breathe or swallow to avoid creating artefacts $^{81}$ on the images. The technician gave these instructions while Patient E was inside the extremely loud MRI machine.

But the instructions are hard to comply with because they're so, well, for example, don't swallow more so than don't breathe. The “don't breathe," they don't say for four minutes, but sometimes if they need

\footnotetext{
${ }^{80}$ Frustration is often evoked by administrative problems, rather than the imaging itself. As Patient G suggests, "That's not medical, that's clerical. And I thought, you morons, you know? You're dealing with sick people. You're dealing with people who need these appointments." Again, it may be difficult to tease out a single precise cause; frustration may be more prevalent when a patient is already anxious about an imaging procedure.

${ }^{81}$ See Chapter 2, page 95, for a detailed explanation of this phenomenon.
} 
to get that.... But the "don't swallows" are a long time. "Okay,

swallow. Relax.” And I'm thinking, no, this wouldn't be a place that

I'd relax. But that's a good word. I get it. But relax? Not going to

happen.

Both patient and technician must comply with the machine's needs, which allow the patient, as object, to co-produce the facts of the disease with the technician. While well intentioned, the technician's giving the patient permission to relax, when the patient is essentially immobilized inside a machine, seems, at best, incongruous.

Frustration is also evoked by long wait-times, not just for imaging procedures, but also the wait-time to get results. Patient G states, "It's a long time to wait. It's probably three weeks, but it feels like an eternity..." Similarly, Patient D says, “And it's that wait. And then you get a phone call, and you've got to go in. And you think, 'Oh, oh it's bad.' And the phone call's only to say it's fine when you go in. But they can't leave that kind of a message." The timeframe, which may feel like normal workflow to hospital staff, feels "like an eternity" for the patient awaiting results, which largely determine diagnosis, prognosis, treatment, and perhaps remaining lifespan. $^{82}$

\footnotetext{
${ }^{82}$ Of the patients interviewed, Patient B is the most vociferous in terms of her multiple frustrations with numerous situations and healthcare professionals. Her frustrations are caused when she feels unheard by physicians, or by a lack of interprofessional communication between different practitioners providing her treatment. In one incident, Patient B reports:

I went for the EMG last week, and I sat for two hours waiting for my appointment. I had an appointment at a specific time, and I sat in a hard wooden chair for two hours. I get in there and say to the nurse, "I'd like you to tell me exactly what's ahead of me." "Oh, the doctor will talk to you after." And I said, "No, excuse me, now, because I have allergies." She said, "I'll get the doctor." He came in and he was furious, just furious. You could feel the electricity. You could feel the hostility. Why hadn't you told us all about this before? The surgeon had it. The hospital has it. I handed it to him and he's just having a fit. He was really very unpleasant. And the net result of it was, I was allergic to adhesives...And when they proposed to do this with really heavy, sticky adhesive and I have very fragile skin, I said, "I'm sorry, we can't do this unless you have some non-allergenic tape that you can use." "Oh, we have." And I said, "May I feel it?" You know, because I didn't want
} 
Several interviewed patients also had either positive or negative emotional responses regarding their time sense from imaging-supported diagnosis to treatment. Recalling her mastectomy in the mid-1970s, Patient A comments, "I was very impressed with the swiftness with which — the quick response with everything that happened to me." More recently, Patient E comments that once diagnosed, he found, "the racing toward treatment is - it's a comfort because it feels like this is probably quite serious and it's comforting to know that it's being done with dispatch..." In spite of the criticisms hospitals sometimes receive about wait-times, Patient E's experience indicates that when dictated by a serious health condition, hospitals can and will respond rapidly.

At times, however, patients also feel rushed. Patient G comments, "I'm always feeling if it's a specialist, I'm rushed; constantly being rushed... you see the specialist, generally because there's something a little bit more involved. And so, quite often I have felt rushed." Interestingly, the specialists who participated in the simulated patient (SP) encounters (analyzed in Chapter 7)

somebody to pull a fast one on me and I'm paying for it. So, he did have somebody bring it in and I could feel it and I said, "Yes, that's okay," knowing that it wasn't going to pull my skin off. He only got halfway through it. He said, "Your feet are too swollen to do the test." As if it were my fault. And I said, "Look, I've been sitting for two hours on a hard wooden chair waiting for my appointment...

It may be tempting to blame the "unpleasant" medical professional for overbooking his practice; however, we have no knowledge of what challenges he might have faced that day. While a clear issue of poor communication is presented in this example, what remains unclear is at what communicative juncture the problem lies. Is it between this physician and his colleagues, or is one or more administrator falling short, or is the integrated computer system within the hospital at fault? By asserting her right to appropriate care for her needs, Patient B reclaims some personal power in this situation; the physician's response is to become "furious." No doubt that Patient B, with her constant need to know, research, question, her insistence that procedures be undertaken in the manner she deems correct, and her demand for common courtesy, is perceived by many of her physicians as a "difficult" patient.

Many physicians, and other healthcare workers, like to clarify the patient's situation, ensuring their own understanding by asking questions; however, the persistent asking of questions also becomes frustrating. As Patient $\mathrm{G}$ declares,

...every time you go to a different doctor...you go through the same thing over and over again. Like, don't they talk to one another? Don't they? They have computers, and they have me on a computer. And it will say we want to give this lady a CATscan and the reason behind it being.... and she has seen this doctor, this doctor, this doctor and this is her medical history, the drugs she's on and everything else. I go through it every time...

Again, anxiety around the medical or imaging appointment may increase patient irritation, so frustration can be easily evoked, even when healthcare workers are simply trying to ensure that they have the correct patient, the correct procedure, and they are trying to do the right thing. 
did not seem rushed upon observation, and, as reflected in post-encounter interviews, did not cause the SPs to feel rushed. This may have been because they knew they were participating in a simulation, rather than dealing with normal practice pressures. Also, specialists are individuals; while some may rush, others may not.

While efficient, Patient F describes his cardiac angiogram as, "an assembly line," and feels like, "I'm number fifteen for the afternoon. Everybody should have the opportunity." Although he had questions regarding the procedure, he "never had any opportunity" to ask them and "no encouragement to do so, or no atmosphere that made you feel you could do so." His description of the environment in which he underwent his angiogram sounds Orwellian:

I was taken to a room which was about the size of a typical school gymnasium, and I would say there were at least twelve, if not fifteen or eighteen, beds. There was a person in each bed and it became obvious to me that they had if not three, there were two rooms where they did the angiograms, and all of these people were being done. And I was told, "Put this gown on. Put your clothes in this and lie down on that bed." I felt I was just another piece of meat going onto a bed. The admitting nurse did nothing to alleviate any fears or answer any questions other than, "Put on this. Put your clothes in that. Lie down on that bed. We'll come for you when it's time." It reminded me of One Flew over the Cuckoo's Nest. Nurse, what was her name...? ${ }^{83}$

\footnotetext{
${ }^{83}$ American author Ken Kesey (1935-2001) published One Flew Over the Cuckoo's Nest in 1962. The story chronicles events in a psychiatric hospital where most of the patients are intent on escaping reality, rather than actually mentally ill. The ward is run with an iron fist by Nurse Ratched, a.k.a. Big Nurse. Her reign of terror over the patients is challenged by Mac McMurphy, who opts for the mental institution over prison. To subdue the unruly Mac, Nurse Ratched has him lobotomized. The book was made into an award-winning film starring Jack Nicholson in 1975 .
} 
If information had been communicated to Patient $\mathrm{F}$ in advance, he might have been better prepared for, and less anxious about, his angiogram. Clearly, Patient F felt objectified and not well taken care of during this procedure. His psycho-emotional care needs were never addressed, leaving him with a sense that, "the efficiency of the angiogram is more important than the person who's having the angiogram."

At the other end of the spectrum, reflecting on her brain angiogram in the early 1980s, Patient B recalls:

In those days, they took you into the hospital so that you had the joy of spending the night in a hospital bed. And then they wheeled you off to the operating room, or the room where they were going to perform the angiogram...I guess I spent the rest of the day in the hospital and went home the next morning. Once upon a time you could actually relax in a hospital. You were there long enough.

The subjective appreciation of time, whether rushed, efficient, or relaxed, informs the way patients feel about their imaging and professional encounters. Depending on the specific circumstances, their emotional responses may range from a sense of relief that their care is moving forward at a speed appropriate to their condition, to a sense of pressured anxiety that their greater care needs are not being met, or that they are not receiving adequate information.

Several patients commented on feeling surprised, or even shocked, either during their imaging procedures or by the imaging results. In Patient E's situation, medical technologies detected the problem before the patient was aware of symptoms, even though it was diagnosed as stage IV cancer. Patients willingly subject themselves to the demands of imaging and other medical technologies, hoping that such intrusions will bring them closer to treatment, to restoring 
their health, and to continuing their lives. Patient E's reflection indicates one of the reasons why

we sometimes fail to think critically about diagnostic imaging procedures, especially for life-

threatening disease states: in that moment, mortality issues are much more pressing than

critically evaluating medical interventions.

Patient $\mathrm{C}$ is surprised when told to report to a hospital for an evening MRI, without being

informed of what area of her body is being scanned or why: "The biggest surprise of all, it was of

the brain stem. I never had a report. Nobody had ever told me I had a brain stem, or why it might

be involved in anything. So that was the mystery of all time." Similar to Patient F's angiogram,

Patient $\mathrm{C}$ was not given adequate information, or opportunity to ask questions; although she must

have provided consent, it is questionable whether this was informed consent. $^{84}$

\footnotetext{
${ }^{84}$ In Patient E's case, healthcare workers were observant and respectful of his responses, his body and verbal language, and willing to discuss his concerns. “...often the people who could see that I was shocked, or when I was shocked, were available to answer my questions, could help mediate the surprises. And they were pretty good about that." While it is tempting to assume that his cancer treatment at a hospital specializing in the disease provided healthcare workers more sensitive to such patients' reactions and needs, Patient A reports a somewhat less attentive experience at the same hospital.

I've been reflecting on this... the numbers are good [regarding her leukemia], but no one's saying, "But how do you feel about it?" No one ever says that to me in just that way, which is very surprising, and in a way not surprising being that I, like many people, am elderly, and I think that it appears to be a given from what I've read and heard from another doctor on television that doctors very often don't like to see elderly coming because they have so many problems...
}

Building on Patient A's observation that perhaps physicians engage differently with this demographic, it is worth noting that Patient $\mathrm{E}$ is the youngest patient interviewed at sixty-nine, while Patient $\mathrm{A}$ is the eldest at eighty-six. Physicians may be reluctant to engage with elderly patients in a meaningful way because of a sense that talking to patients with multiple co-morbidities, whose thoughts perhaps wander, is time-consuming. It is true that elderly patients may have multiple health issues, and this complicates their care. In addition to physical health issues, they may also have decreased hearing or vision, making communication more difficult. Additionally, elderly patients may suffer from mental health issues, such as dementia, again, making it more difficult to ensure understanding. These are potentially challenging patients, ones who may require more time. Yet, only one of the seven patients interviewed for this research took inappropriate advantage of the open, semi-structured nature of the questions, and the interviewer's time, by constantly diverting from the interview task. The interviewer struggled to get her to focus and eventually, had to find a way to leave courteously after more than two hours. So yes, an argument can be made that some elderly (or younger) patients can take inappropriate advantage of a physician's time; however, this field research suggests that those people are the minority. Perhaps professionals fear that asking an open-ended question, such as, "How are you today?" or "Do you understand?" may open a Pandora's box of troubles, many of which may seem irrelevant to the patient's care. Such a simple act of empathy, does, however help patients feel that their healthcare providers are concerned about their welfare. 
Some sense of vulnerability was communicated, either overtly or subtly, by nearly all the interviewed patients. As Patient G observes, “...you are vulnerable if you're sitting in the hospital." Some noted the lack of privacy while awaiting imaging; for instance, Patient B says, "I suppose I was the $125^{\text {th }}$ patient of the day and I was sitting in a hall. I had no curtain around me, I had somebody staring at me from across the hall, feeling very exposed." Patient E comments: 'I don't have any control over what's going to be seen here [on imaging]. I think I'm doing all right, probably am, but it's possible I'm not. Maybe I shouldn't be in such a hurry to come to the six o'clock in the morning appointment." This psycho-emotional sense of vulnerability is also reflected in Patient A's comment, "Well, and I think too, because of the way people feel about medicine and hospitals, and their fear of being sick or dying, you know all of that plays into it, doesn't it?" In Patient F's case, the vulnerability is more physical: "I saw the girl sitting beside the machine that they hook you up to, to move the blood from your heart to...the machine does ${ }^{85}$...but I've thought since, you know, that job she is doing, she literally has your life."

While negative emotions, such as fear, frustration, shock, and vulnerability are present at some point during all the interviews, patients also reflect neutral or positive emotions. Although Patient F's angiogram experience is terrifying, he says he feels calmer in situations when he has opportunity to ask questions. Several patients mentioned that they reach a relaxed state during long imaging sessions by reciting lines from memory or practising meditation. Such practices evoke a sense of calm, of being out of their bodies while things are being done to their bodies. Patient G reports, "Well I know people who have had them [scans], and they're not openly religious, but they do the Lord's Prayer. You know it's not because you want to pray to God, it's because you want to take yourself away from the situation that you're in." She herself mentally

\footnotetext{
${ }^{85}$ This is in reference to the cardiopulmonary bypass (CPB) pump, or heart-lung machine, Patient $\mathrm{F}$ was hooked up to during his quintuple bypass surgery.
} 
rehearses acting lines or upcoming tasks; "it's an out-of-body experience," she laughs. Patient B describes her bone scan as "a very tranquil experience. Long, but tranquil." She attributes this to "feeling that I was in the hands of somebody who was very skilled," so "there was no feeling of threat or concern." Patient A, who meditates regularly, says,

So, when it's time for anything, I can do a lot of visualizing ahead of time that can be kind of negative and going through it all in advance. Even if it's going to the dentist for a root canal, I go through all of that. But when the time comes, whoosh [she goes into a meditative state]...I know what to-you know the jaw's yawning and relaxing the jaw and everything. So, I'm not inclined with machines to look and see what's going on if it is available to me. It's not that I'm frightened to look necessarily, or not interested, but I'm more interested in doing my—closing my eyes and letting the light and love flow through my body, every cell, etcetera. And I can do that quite quickly now...Very useful, very useful—and very often I find things extremely funny.

Laughter is not something one expects from patients during imaging or other medical procedures, but given the right state of mind, it is possible to be this relaxed. Patient A's positive attitude helps her through challenging health situations, and also encourages physicians and other healthcare workers to engage with her; they take their time, talk to her, and want to help her. Although Patient E reports that his MRI experience was "shocking," he continues, "I just try to take myself away. I'm sort of musical, so if I can get a rhythm to the sound of the - chicka, chicka, chicka, chicka — whatever the noise happens to be, if I can convert it into something musical, then I've got a sort of instant musical composition going on." While it is possible for 
patients to calm themselves in medical situations, it may take a conscious effort. The relaxation methods these patients mention are things that they themselves devise to cope with their circumstances. They find ways to dissociate their conscious minds from their bodies, somewhat reflective of the way imaging technologies separate their bodies from their subjectivity.

Another common positive emotion interview participants report is a sense of relief at the conclusion of an imaging session, or sometimes upon hearing results. As Patient G comments, “Just glad it's over. There's no pain. Just want to get my clothes on.” While Patient E gives more detail about his exit from the session, he ends with “...for me it's like, thank goodness I'm out of here.” Similarly, Patient D says that when his MRI ended, “...I felt quite relieved.” His anxiety increased while waiting for results; however, once the follow-up appointment was booked, “...it was a relief in the sense that it was somebody there that would talk to me and tell me exactly what they saw and what they thought it was..." In his case, the lump was benign. Subsequent to the negative findings of her brain angiogram, Patient B comments, "I had no brain lesions of any kind, so that was a relief." Subsequent to a later imaging procedure, she indicates, "It was actually comforting to find that there was an actual reason why I was having pain in my feet is because there is blood pooling there, which is an indication of infection of some kind, possibly just disintegration from arthritis." Not knowing, not understanding what one is experiencing, is challenging; finding an answer, even when it is bad news, is, on some level, a relief. Although Patient C's X-rays show that she had lung cancer, she says, "that was one time when I really blessed the machinery." Answers evoke a sense of gratitude for the availability of diagnostic technologies. Reflecting what many of the interviewed patients thought, Patient A says, "I felt good in every case because of the results. Thank goodness they have these images now because at one time they didn't, and now they know so much more." Patients are clearly glad when 
medical technologies yield results that move them towards diagnosis and treatment, even when the imaging experience or treatment are difficult. This sense of relief is similar to Blaxter's experience that the images were, "informative and even reassuring" (CVP 776).

Compared to still images, like X-rays, real-time imaging, like angiography or ultrasound, presents a different sense of patient experience and body conception. As Radstake acknowledges, "patients regard the images on the monitor with mixed feelings: awe and fascination on the one hand, fear and avoidance on the other" (92). Patients F and A both comment on their real-time imaging experiences. Patient F reports, “...they said at one point, 'Oh yeah, there’s the heart,'... and she said, 'If you'd like to look at it, it's on the monitor on your left. You just turn your head.' I said, 'No!' There was no way I was going to look at it." Although he is encouraged to watch the onscreen images of dye dispersing through his cardiac arteries, Patient F says, "I kept my eyes closed the whole time." Similarly, although the screen is conveniently located for patient viewing, Patient A also avoids the images produced by a scope. "I "I think it was pointed out to me, you can watch if you wish." When asked if she took the opportunity, she responds, "I didn't take it, no." When asked why she chose not to look at the real-time images, she responds:

Well, because I say that's what I do. I close my eyes and-the same if I'm having blood taken; I know I can look and watch. I simply close my eyes and am very relaxed. There is no rigidity. I feel that's my part in it. But I can always check in advance on the computer and get information. But I'm inclined to keep things as positive as I can in my own mind and not think, “Oh, dear they're going to do this and that..."

\footnotetext{
${ }^{86}$ Patient A refers to this procedure as a "stethoscope," perhaps because it was simply called a "scope." Given her description, this was likely cystoscopic imaging used to investigate the urinary tract and bladder; however, at one point, her description also sounds like a colonoscopic investigation of the bowel.
} 
While Patients $\mathrm{F}$ and $\mathrm{A}$ both choose not to look at the screen during real-time imaging, their emotional states and reasons for avoidance are very different. Patient $\mathrm{F}$ is terrified, while Patient A is calm and relaxed. In fact, she is so relaxed during this procedure, she reports, "...you can laugh about it, at least I can, and they [healthcare workers] seem to go along with it." In spite of what many consider a physically and psycho-emotionally uncomfortable procedure, she finds the ridiculous humour in having tubes sticking out of various orifices. Although Patient A does not engage with her real-time images, she has no reluctance in looking at still images: “Oh, I've got a lot of images of myself [laughs]. I like to have those images." Her avoidance of real-time images is because it interferes with her routine to stay relaxed during medical procedures.

This section examined the emotions older patients report before, during, and after imaging experiences. While many of these emotions are negative (e.g., fear, frustration, shock, or vulnerability), neutral or positive emotions are also reported. Patients' time sense evokes conflicting emotions, with some reporting a sense of relief that diagnostic imaging results mean the beginning of active treatment, while others finding that wait-times for appointments or results are too long and increase anxiety. The most palpable positive emotion expressed was relief or gladness, either because imaging results were negative and ruled out anything catastrophic, or because imaging caught something early, propelling the start of treatment. Several patients expressed positive emotions of calmness or relaxation intentionally evoked as a coping strategy, through their own effort using the mental repetition of memorized lines, prayers, or mindfully practised meditation. 


\section{What physical sensations are described?}

I said, "Can't you give me something for the pain?" And he said, "It says in the book it doesn't hurt."

\section{Patient C}

Imaging is generally considered painless; however, most interviewed patients reported unexpected physical sensations. This section examines physical sensations experienced by patients, separate from their medical conditions, and directly or indirectly related to imaging procedures. The most commonly reported physical sensations are of pain and feeling cold, although other sensations are reported by some individuals.

While the imaging itself may be painless, demands of the equipment sometimes cause pain. Several patients describe difficulties lying on the gantry during imaging. Patient $\mathrm{C}$ reports “...you're on your back, and that may not be your comfortable spot... That is an agonizing position for me to be in for any length of time...it was a miserable experience." ${ }^{87}$ Similarly, Patient A also finds the gantry an uncomfortable part of the imaging experience: "You're so flat, so flat." She also comments that the gantry is so narrow that it is a struggle to stay on it, even for a small person like herself. In addition to the physical stress of lying on the narrow gantry, ...your arms had to be back up over your head. You're flat, my thin little arms back over my head and this cylinder that went over my chest area. I was left alone like that for, I don't know, for me it's like half an hour, but it could've been less than that, but not too much less than that, while this little thing is going back and forth. The horrendous thing about that was that my arms were back and almost

\footnotetext{
${ }^{87}$ Patient $\mathrm{C}$ describes this scanner as a "doughnut thing," but denied that it was either a CT or MRI machine. Clearly it was a scanner of some sort, and some newer models do use a doughnut-shaped structure, rather than the claustrophobia-inducing tube.
} 
immediately they started to shake and the strain on them was

incredible. That was the most uncomfortable experience, bar none, that I've had... ${ }^{88}$

When asked whether she had access to an emergency call button during this procedure, Patient A comments, "There would be no way you'd be able to touch it from that position that you were in." While emergency call buttons may be helpful for patients in sometimes claustrophobic or difficult circumstances, they may not always be accessible.

Patient G reports, "I have the bad back and they did a complete skeletal X-ray of me. Well, I couldn't get up off the bed. I mean, it was because of my back. I had two broken vertebrae in my back, and I couldn't get up, I couldn't roll over.” The technician, a small, older woman, was unable to help. Patient G continues, "Some of us are not able to move; if you've got a couple broken vertebrae in your back, you can't move. The pain is unbearable." So while the imaging itself may be painless, the simple act of lying on, or getting up from, the gantry may be excruciating for some individuals. Eventually, Patient G's husband was called to help. In another circumstance, in which the technician was "a big guy" who was "very aware," he offered a strong arm for Patient G to grab onto, so "he gets me up immediately. There's virtually no pain."

Not surprisingly, Patient E reports, "I was not comfortable with my head being locked into place, my arms behind my head. But I understood why it was happening..." While able to rationalize his experience, he also acknowledges that he has no mobility issues that might make the situation worse. In his experience, "Nothing hurts. Sounds from the MRI, they hurt in a way, but I think the radiation screwed my hearing enough that it didn't bother me as much, but nothing hurts, and I'm grateful for that; nothing that the treatment did. Well, in a sense it hurts a

\footnotetext{
${ }^{88}$ From the patient's description, this was likely a CT angiogram. As with a regular angiogram, dye is introduced into the coronary arteries, but the imaging uses a CT scanner.
} 
lot...." Elsewhere, Patient E reports on the terrible adverse effects from radiation and chemotherapy; clearly, the treatment hurt his body a great deal. Although this radiation exposure was for treatment, rather than imaging, he also underwent numerous imaging sessions.

Discussing his MRI experience, patient E reflects,

The voice, the sounds for me are-they're very unpleasant. They're like the sounds of jackhammers, like walking along the street where they're doing construction. Real loud. Like the police officers who get paid extra duty to stand and divert traffic from areas that are-and they have earplugs in. They probably know exactly what the experience of an MRI would be like. It would be good training, because it's a shock initially.

The volume in an MRI, even with ear protection, is alarming. For some, it may be an effort to stay calm surrounded by such noise levels. As well as repeated MRI and CT scans, Patient E also experiences endoscopic examinations to assess the progress of his tonsillar cancer and treatment. They stick a long unpleasant tube down nose, into my throat. Make me say things that really hurt. Not the content, but I mean speak with this thing down my throat which has basically got a light at the end of it, and it's not pleasant at all and actually hurts. And they're looking for trouble.

Given that his throat has already undergone burning radiation, and the patient is vomiting due to chemotherapy, this unpleasant procedure is particularly painful.

Several patients also report feeling cold during imaging. Patient G says that during her bone scan, "you can feel the temperature change when it goes over you." During other scanning 
procedures, she reports, "I remember being cold. Every time I have it done I'm freezing cold in those rooms." She then rationalizes, "But it's probably because it has to do with the equipment.... Of course it was the winter..." While other patients mention the cold, none of them attempts to rationalize this aspect of their imaging experience. During an early mammogram, Patient A reports, "I remember the room being icy cold. I have never had anything like that since, where everything is very, very cold." For Patient E, the cold is experienced both prior to and during imaging: "And then you get moved to a place where you get into a gown — where I got into a gown. A little changing room; it's kind of cold. And then you wait in a different area with one gown, so it's chilly and a little embarrassing, and an opportunity to get a look at other ill people." After this chilly experience, Patient E describes, "The rooms that are used, the rooms where the technology lives, are anything but sort of warm, friendly, home-like environments.” Perhaps hospital thermostats are set for fully clothed employees, or to reduce bacterial growth, rather than for patients in thin gowns, who are unwell, and possibly older.

Interview participants report a few additional unexpected physical sensations. Patient B, who has had numerous MRI imaging sessions, experienced one unique late-night session.

There was something that was making my arms twitch, which was very strange; making my body twitch... when the machine starts you twitching, you start to get a little — anxious about it.... That made me feel the machine was perhaps not properly tuned, or that there was maybe more current being used than was needed.

Although Patient B reported this strange sensation to the technician, the technician did not feel that it was in any way odd. Muscle twitches, or fasciculations, have been reported by MRI patients since advances in the late 1980s created more rapidly switching magnetic fields. A 1989 
study of the peripheral nerve stimulation (PNS) that causes such twitching, indicates that it is related to the switching rate of the magnetic field established by the MRI machine; rapidly switching magnetic fields stimulates nerves and creates a twitching sensation in subjects. This study also suggests that standards be adopted to minimize such adverse effects of MRIs (Reilly 108). Patient B's conjecture that the machine was not set up correctly is accurate.

Patient A describes an imaging procedure that caused her to feel very dizzy, although from her description, it is unclear whether this machine turned around her, or it turned her body. One of the ultrasounds I had after barium, which I've had a number of times, I get very dizzy when they upend the machine. You know, they turn it. It's like something like what you would get in a fairground. The thing goes around and I- even as a child I couldn't sit on a swing without getting dizzy. I mean it's still a swing, a motionless swing. While Patient A was dizzy enough to require assistance afterwards, the type of machine described is unclear. As is clear from this chapter, each patient is unique in his or her experiences, and also in his or her responses to those experiences. ${ }^{89}$ For instance, while some

\footnotetext{
${ }^{89}$ Patient E's radiation treatment, lasting fifteen-to-eighteen minutes each day for over thirty days, required considerable preparation. To ensure accurate radiation targetting, based on a CT scan, a mask was created to anchor his head for treatment.

So then I'm in a room where I'm told that I'm going to have a mask made. And again it's like sculpture class. I like that. I mean, I'm artsy, and it's like this is kind of fun. Somebody's going to make a mould of my head, neck and upper shoulders. And I understand that, when it hardens, will contain me when I have the radiation. And it's a little scary to have this - it's like cat-gut from a tennis racket which they soften and then put over your head. And it's soft and warm and they can stretch it and it takes the exact contours, but it sort of pushes your nose and that, over your eyes. A little bit confining really. It translates. I kind of wish there were eye-holes. I mean I can sort of see. And it would be great if I could move my mouth and breathe a bit easier. And this isn't treatment yet either. But it's a little scary. And then they lock you onto the table because it's necessary for the physics to work, to get the beams, the radiation to get exactly where it needs to go, and they don't want you moving around each time.
}

Making the mask, a very physical step between imaging and treatment, was both entertaining and scary for Patient E. To his relief, both eye and mouth holes were cut into the mask. Over the duration of his treatment, as his body 
patients report that transvaginal ultrasounds, in which a probe is inserted into the vagina, are unpleasant, Patient A reports that she rather enjoyed hers, laughingly saying "What's not to like?"

While this section describes physical sensations, Patient F describes what might be interpreted as an omission of a desirable physical sensation during his angiogram. ...all of a sudden I heard this voice that said, "Oh, yeah, there's the heart." And I kind of wondered why? Why would he be saying that? But just all of a sudden to hear this voice. As I say, he could've put his hand on my shoulder. He could've introduced himself. I suppose he was doing the procedure to someone else while I was lying on the bed, so he couldn't come out and speak to me. I don't know, but just that moment of coming and introducing himself.

In this passage, Patient $\mathrm{F}$ describes two accounts of a lack of physical sensation: one is the lack of sensation of the procedure, during which he was somewhat anesthetized; the other is the lack of physical contact with the unknown physician doing the procedure. While the first is a welcome relief, the other is perceived as threatening. Having another person, regardless of his professional status, doing things inside your body, around your heart, can be frightening, and has the potential to create a strong sense of vulnerability and fear. As Patient F indicates, simple actions like the physician introducing himself, or placing a reassuring hand on the patient's shoulder, might have reduced the patient's fear.

Although unexpected physical sensations vary widely between patients, the most commonly reported were those of pain and feeling uncomfortably cold. Other notable sensations 
include muscles twitches and dizziness. In one case, the patient commented on the lack of physical contact by way of an introduction to the physician handling an invasive imaging procedure.

\section{Conclusion}

This chapter has reviewed and analyzed information from seven interviews with older patients, interrogating these texts with two primary questions regarding their emotions and physical sensations before, during, and after their imaging experiences. Although most of the emotions were negative, sometimes positive or conflicting emotions were also reported. Many patients have developed unique ways to deal with the emotional stresses of imaging appointments. Although unexpected physical sensations vary widely between patients, the most commonly reported were those of pain and feeling uncomfortably cold. Other notable sensations included muscles twitches and dizziness.

Although imaging technologies may be optimized for technicians and medical practitioners, clearly they do not always provide an optimal experience for older patients. In some cases, consent may not have been fully informed, violating ethical considerations for imaging and other medical procedures. Patients' emotional or physical discomforts were often unacknowledged, even when the patient gave clear verbal or non-verbal cues. This analysis begins to answer the larger questions posed in this dissertation: in practise, biomedical technologies, and their requirements for successful imaging, can sometimes take precedence over patient comfort and patient-physician communication. An analysis of patient communication with medical personnel, and patients' rationalizations about these relationships, appears in the following chapter. 


\section{Chapter 5: Older Patients' Communicative Experiences and Rationalizations}

...now my concern about the machines and numbers results is that they

have the information that they need to know, but do they know what I

need them to know?

Patient A

This chapter continues the analysis of patient interviews initiated in the previous chapter.

Moving into the patient-practitioner relationship, here the focus is: What communicative experiences and rationalizations emerge about imaging in the medical context? Patients tend to communicate with their doctors or other healthcare workers (formal information channels), as well as other patients, family, and friends (informal information channels), when seeking medical information; they may also resort to the media. This information, coupled with the patient's own ideas, may range in accuracy. Especially in times of stress, like those caused by illness, patients may want to listen to, or tell themselves, stories to rationalize their experiences, and in an effort to find comfort. Due to the hospital context, many interview participants told stories about doctors and other medical or administrative personnel. While some of these stories are unflattering, they give a snapshot of the ways patients perceive professionals in the healthcare context.

\section{Formal Information Channels}

Halfway through our meeting, he closed up my file and said, "That's enough," not answering my questions. ${ }^{90}$

\footnotetext{
${ }^{90}$ Patient B is the most vociferous in her complaints about personnel in the medical context. She suggests, "It's in my nature to want all the detail, and generally it's available to you if you have a good rapport with your service provider. ... I mean I'd like to feel that I can ask, and that I can be told." Increasingly, however, she finds that physicians distance themselves from their patients in various ways. Patient B suggests that physicians, "...should be
} 
Patient B

Unlike Patient B, Patient E feels that although "the information was shared...it never made any real sense to me." Information about his condition is usually, "read off. You know two sentences on a piece of white paper" that is not helpful or informative for him as a patient. Patient E is a visual learner; an opportunity to view the images might have been more informative for him, but, "It was never offered." His physician, and others in the field research, tend to assume that patients either will not be interested, or will be unable to understand medical images, but this is an assumption, and patients should at least be offered the opportunity to view their images.

Patient A cautions practitioners not to make assumptions: "Just because you've sent someone to a specialist doesn't mean the situation's been taken care of." Because family practitioners act as gatekeepers to specialists, they may consider their responsibility and involvement in a health situation to end once the patient is sent to a specialist; however, this is not always the case. Patient $\mathrm{F}$ found that the cardiac specialist responsible for his angiogram provided enough information to create fear, but not enough to adequately explain the situation to the patient. Unless patients book a follow-up appointment after seeing a specialist, or return to their family practitioner still complaining of an issue, a possibility exists that the physician will not initiate follow-up.

Generally, however, Patient A suggests that her imaging experiences, and the communication around them, have been very positive: "I felt good in every case because of the results. And the two [in] 2011 where I heard about the [...] operation, I felt good, elated that the

careful about talking down to a patient, or giving trite answers to questions that - in other words, brushing the question aside, or making that person feel that they don't have a right to, or embarrassed that they've been asked a question." She is quite assertive in her need for information. As an example, "...you can't just look at a patient and say, 'I think you've got peripheral neuropathy'... without telling them what it was, and then leave," an experience she has had. This particular physician's approach, using the technical language of physicians when speaking to a patient, provides an example of a practitioner not using patient-centred communication. 
doctor could do something about it and it was going to be soon...I've always felt that the information has always been there for me." Even in terms of preparation for imaging and other procedures, Patient A feels very positive: "The printouts that you get ahead of time, in every case are very thorough. And always there was a number there that you can call if you have any questions ahead of time. So, no, I think it's a super job done." 91

Unfortunately, Patient B finds multiple communication gaps between patients, physicians, and alternative medical practitioners. When joint replacement surgery left her knee "forever painful," she had a reassessment, including X-rays. While her physician and surgeon say, "there's nothing we can do for you," her osteopath detects, ...a one-inch gap below the bottom end of the lower prosthesis and the knee. The shaft does not go....as far down the leg as it was intended to, and therefore I have this problem. And it's just great for somebody who has disk problems in three areas of the back; it throws everything off. That's my worst medical nightmare because there's no end, and there's no solution.

${ }^{91}$ Arguably, the patient's positive attitude has a positive effect on her medical situation; doctors, and other healthcare personnel, respond positively to her.

...the doctors and the one who did the surgery in the first place, he certainly let me know from the get-go that I would do because I was very positive. And I did. So, that was a good feedback to have from him right from the get-go, you're positive and all of that. It sort of gets you set on your path. You know, you've been given a pat on the back, and it's part of the way you approach things I suppose too, but a recognition that I think is awfully good to have that encouragement. It stays with you...

While some physicians may scoff at the power of positive thinking, some patients find it helps them through the difficulties of dealing with insecurities about their health situations. Patient $D$ also expresses the advantages of thinking positively in medical situations, and feeling positive about one's practitioners: "If you're uncomfortable going into the process or the procedure, then it's going to be harder for you to come out in a healthy state, whether it's a healthy state of body or mind, because you're not positive going in. It's easier to go in saying, 'I like that person; I trust their judgement..." While circumstances may not always allow patients to choose their physicians, the patient-physician relationship is an intimate one and patients must feel that they can trust the person or people providing their care. 
Patient B, who was an avid golfer and walker, describes suffering from a great deal of pain and reduced mobility since her knee replacement. She feels that the post-surgical X-ray findings, as interpreted by her osteopath ${ }^{92}$ have been ignored by her doctors, and that her doctors refuse to resolve the issue.

After stubbing her thumb when a city bus braked suddenly, Patient B goes to the hospital where she waits four hours before being sent home and told to return if her thumb worsens. Three days later, she returns, her thumb badly swollen. After another four-hour wait, she is given X-rays, but no results. She is given a doctor's phone number and told to call him; she finally sees him two weeks after the accident. In the interim, she has an osteopathic appointment. Upon palpation, the osteopath declares, "Your thumb is broken." What makes this incident remarkable for Patient B is that the osteopath, "was the only person in this whole exercise who actually palpated the thumb.” In spite of repeated hospital visits, and lengthy waits each time, “...nobody touched my thumb. In four hours, in the first four hours at the [hospital] nobody touches the thumb. When I come back with the thumb even more swollen three days later...Nobody touched it." Patient B perceives that not being touched, not having the injured thumb palpated, is poor

\footnotetext{
${ }^{92}$ Patients, dissatisfied with the medical profession's answers, may persevere in their quest for health by turning to alternative healthcare practitioners, like chiropractors, osteopaths, naturopaths, massage therapists, or others. Although alternative treatments can be expensive, and may not be covered by insurance plans, in some instances patients find them more helpful than traditionally sanctioned medical practices. As English and medicine scholar David Morris suggests, alternative therapies reveal "an impulse to recover lost knowledge about health" that "respects the wisdom of nonscientific traditions of healing" (5). When the scientific approach fails a patient, alternative medicine retains hope. One difference between conventional and alternative healthcare is that, as indicated by Patient $\mathrm{B}$, alternative practitioners often spend more time listening to and interacting with their patients. Patient A suggests:

Listening is just absolutely fundamental too. You wonder why some people never, who are doing medicine, or who are in communication with other people, where messages are received and given are so important, why they don't make that connection. I don't understand. It's very difficult for me to understand that because there's nothing more important that that ability to listen, and to hear what is being said.

Patients, especially elderly patients, often feel rushed and unable to fully express their medical concerns. Even when they succeed in expressing their concerns, they may not feel heard, or that their concerns have been acknowledged. Fundamentally, this means that they may not feel that their healthcare needs are met.
} 
quality care, and an unwelcome change from what might have happened when she was younger.

Her experience also indicates the strong reliance on diagnostic imaging, rather than hands-on

assessment, in hospitals.

Patient B also comments on healthcare providers whose communication skills she considers sub-par. These instances often relate to practitioners she perceives as not EnglishCanadian. Not greeting a patient or shaking her hand, which might be due to cultural restraint or concern about spreading germs, she considers "extremely rude." In another instance, during MRI imaging, she "had a technician whose command of the English language was somewhat abbreviated." While Patient B might be considered prejudiced, a point can be made that when undergoing a potentially frightening procedure, it is desirable to be able to communicate successfully with the technician. Patient B comments, "you really don't get any comfort from the person who was doing the MRI because her command of English is not all that good...."93 Prejudiced or not, this incident points to a potential problem in patient-practitioner communication. $^{94}$

\footnotetext{
${ }^{93}$ Conversely, Patient A's hospital experience causes her to reflect on how lucky she is, compared to some patients. For instance, she says, “...you just have to walk into a hospital and see those corridors absolutely filled with people from different countries who, you know - how lucky I am that I speak English, in an English country, instead of being an immigrant in another country." Certainly, Toronto-area hospitals are multi-lingual. It is common to see English-speaking adult children translating and advocating for their aged parents who speak limited or no English. What happens to those with no family here? Reflecting on the activity in waiting or emergency rooms, Patient A says:

Even though we have, I guess, one of the better systems, we could see the changes and the crowds of people in need. It's incredible. So, you do get to a point where you can see not only your own needs, but you can see the needs and the way the other person feels too. If you expect to be listened to yourself, you have to listen to the doctor and the other people who are needing help, and be aware of that. I think it's such a learning situation all round.
}

Given Canada's culturally diverse and aging population, the patience and understanding Patient A exhibits in this passage, the necessity for everyone to view waiting for medical care as a "learning situation," will become more and more necessary. A recent study by the Canadian Institute for Health Information indicates that central Toronto hospitals have emergency room wait times of one-and-a-half to six-and-a-half hours; Winnipeg's Grace Hospital clocked the worst reported wait times in the country, at over nine hours (CBC).

${ }^{94}$ Complaints and kudos about healthcare professionals surface in other interviews too. Patient G says, "Sure I have lots of complaints about the medical system, but not the doctors, not the regular nurses. I emphasize that, and not the technicians. I'm happy with those three." Where she finds shortcomings is with administrative and weekend nursing 
Not all medical communication is problematic. Patient G says, "I always felt I got more

information from my family doctor than the specialist." Similarly, Patient D states:

They [the results] were better explained by my family doctor as a

matter of fact. And why? I would say that is that I was probably in a

better frame of mind with my family doctor. I am familiar with my

family doctor. And, strange as it is, I rely on my family doctor,

physician, greatly to, sort of, give me the ins and outs of the specialist

if I'm going to see one and what they would be doing. And he knows

staff, whom she sees as disinterested and ineffective healthcare workers. In general, she is sympathetic to nurses; "I can't imagine why anybody would want to become a doctor or a nurse, and nurses aren't necessarily treated that nicely." She also suggests that sometimes nurses are as knowledgeable, if not more so, than doctors; and yet they still must bow to physicians for their education, title, and patient responsibility. Patients, and perhaps nurses, sometimes have unrealistic expectations about doctors' knowledge and power. Patient G appreciates when "doctors are honest, you know, 'I know all about the bowel, but I couldn't tell you a thing about the lung,' and they'll admit that. Whereas we think, 'Oh, he's a doctor. He must know everything.' But that's not the case, and it's not really realistic either." Patient G also recognizes that although nurses are required to defer to physicians, when they say, "'I think maybe you should talk to doctor so-and-so about that first, I'm not really qualified to do that,' it still scares you. What can't you tell me? What's wrong?" Depending on the situation, the limits of nurses' professional authority may unnecessarily trouble patients.

Patient G's issue with weekend nursing staff was with her perceived lack of care at a particular hospital. I needed assistance, and if they paged her once, they paged her twenty times, not just for me, but for other patients, because she would have other patients. Like, where the hell was she? And there should be a mechanism that we can - that if you do complain, and I have a friend who volunteers at [this hospital] and there's been complaints about certain nurses. Well they take it out on the patients and a lot of them are older people, some of them are not in very good shape, so they [the patients] hesitate. And I guess after they have seen their nine-hundredth patient they [weekend nurses] think, "Oh God, I've got to go through this one more time."

When contacting the hospital ombudsperson is suggested, Patient G is dismissive, saying, "I'm sure the ombudsman has a thousand people that are complaining — not always legitimately." Although many hospitals have an ombudsperson, fear of reprisals targeted at vulnerable family members often stifle feedback, reducing possibilities for positive changes.

Although I drafted a letter to the ombudsperson of this same hospital, I did not send it, because I was concerned about potential adverse affect on my mother's care. The issues were serious and impacted her care, as well as causing additional unnecessary stress to the family. In one case, her room was changed on a daily basis over a week-long stay, so family phoning her received no answer, and those visiting found an empty bed. Administrative staff were not consistently aware of her location. She fell several times while in the hospital due to inadequate monitoring. On release, she was discharged to await return to her retirement home via ambulance, when one became available. Family was not informed of her release, or we would have picked her up. She waited several hours, becoming progressively colder, until she was finally returned to the retirement home exhausted, shivering, and mentally confused. Family and the retirement home had spent several panicked hours trying to locate her. Along with physical problems my mother developed in her last few years, her care was complicated by dementia. Her lessthan-ideal experiences in the hospital context are similar to issues raised by some interview subjects. 
enough to do it in laymen's terms. ...Just simply because with my own doctor, he knows me. He knows how to tell me things. And that's just through conversation back and forth, so feel comfortable, and I suspect if he's going to use big technical terms, he says, "No, [this patient] won't know this. I'll give him some other terms." It's that type of a relationship that I find that for me it puts me at ease.

Patient D's experience speaks to the importance of having a primary care physician, rather than relying on walk-in clinics or emergency departments. An effective primary care physician oversees and coordinates imaging appointments and other specialists, which can be numerous for an older patient with multiple health issues; a primary care physician can also provide understandable explanations to patients regarding imaging or other test results. Additionally, primary care practitioners offer continuity of care for patients and, over time, develop an intimate knowledge of that patient's physical and psychological health. Unfortunately, the increase in many elders' healthcare needs often coincides with their long-term primary physicians' retirement, disrupting the professional relationship and that continuity of care. As Patient A relates:

I've had a doctor for thirty, thirty-five years that was wonderful. I found it very difficult after because apparently he had left instructions, when he became ill that, his patients be handed over to staff doctors, but I wasn't. I was given — every year I would have a new doctor. And finally I said to them, this is the third doctor that I've had in four years, three years. And I said, I've been here for thirty-five years! And 
they were very pleasant, all of them. But, that change at this age is difficult.

During these interviews, other participants also commented on losing their primary care physicians due to retirement, illness, or leaving the practice area. Although physicians usually tried to transfer their patients to reliable caregivers whom they trusted, things did not always work out as planned, leaving patients in difficult circumstances. While change can be challenging for any patient, it is particularly difficult for older patients with decades-long relationships to their primary caregivers. As Patient D suggests, developing a long-term professional relationship between patient and practitioner provides a sense of trust and strengthens communication.

Such advantageous professional patient-practitioner relationships may be challenged by advancing medical technologies. In addition to being misplaced in a hospital corridor, ${ }^{95}$ Patient $\mathrm{C}$ also expresses concern regarding the (over)use of new technologies.

I think that there's a tremendous reliance on these new-fangled, as it were - I think they have a place, but I'm wondering if sometimes they're not being overused, because with every use of outside aid, young doctors miss-I've never been asked, when you were a child, you know, my kids called it the "ahh-stick," the tongue depressor. The first thing the doctor looked at was your tongue, and evidently you can tell a great deal. During that period, when I was in the hospital

\footnotetext{
${ }^{95}$ Patient $\mathrm{C}$ describes this as "the time they lost me." A day after esophageal surgery, she was taken for a contrast MRI. "And then it was all over and the porter took me back, but he took me to the wrong floor and left me in front of the desk." Eventually, her worried daughter located her. By this time, Patient C was frantic. "I kept saying, 'Can somebody please help me?' and nobody paid any attention to me at all. And I must've been there for, I don't know, it seemed a lifetime, but a fairly long time by the time [my daughter] checked all the floors (laughs). But that was a bad thing." While this is similar to the kind of administrative error mentioned by Patient G, in Patient C's case, she was still weak from surgery and it created enormous and unnecessary patient and family anxiety.
} 
with the esophageal thing, nobody looked in my mouth—ever. ... this

is an art that's being lost, I think, is being able to see something on the

tongue. Very simple thing.

Some of the discussion in Chapter 2 indicates that at least some practitioners share Patient C's concern. After her esophageal surgery, her tongue swelled - an unexpected complication. For a long time, she was unable to eat and lost forty pounds. Surprisingly, no one inspected her tongue or mouth to assess the severity of the swelling. Similar to Patient B's incident with her injured thumb, Patient $\mathrm{C}$ was left without answers, and with a sense that none of the professionals cared enough to investigate or attempt to resolve what, for the patient, was a serious health concern. In her case, perhaps a hands-on physical examination was seen as ineffective, or an inefficient way to gather diagnostic data.

While Patient $\mathrm{G}$ comments on the lack of administrative efficiency, ${ }^{96}$ Patient $\mathrm{F}$ finds the angiogram experience overly efficient. As previously noted, he describes it as "an assembly line.” In trying to rationalize his experience, he says,

\footnotetext{
${ }^{96}$ Patient $\mathrm{G}$ comments on poor administrative and telephone communication. On one frustrating occasion, she was left a phone message regarding a medical appointment and asked to return the call to confirm. When she phoned back, she heard an outgoing message indicating that the office was closed until morning. She phoned several times the next day, receiving the same message. Finally, she was informed that she was dialling the wrong number, and given an alternative number. Although someone answered, she was told it was the wrong number. When she arrived at the hospital to check in for the appointment, she is told that the doctor in question does not work at that hospital. After further insistence on her part, she was finally told that she was correct and she could see the doctor. Of this incident, Patient G says, "That's not medical. That's clerical. And I thought, you morons, you know? You're dealing with sick people. You're dealing with people who need these appointments." For her, the administrative problems boil down to, "the left hand doesn't know what the right hand is doing." The unfortunate consequence of this is that it may erode patient care, compliance, and confidence.

Patient $\mathrm{G}$ rationalizes her irritation about both weekend staffing and administrative gaffs, saying, "I know it's all about money." She adds, "...the clerical staff-a lot of mistakes are made. It's more attitude than anything, and I know they're busy. We all are. There's a lot of waste, a lot of waste." Hospitals struggle with efficiency. In the last few decades, this has translated into increasing technology and stable or decreasing staff. Hiring contract weekend staff, rather than more costly regular staff, may be an attempt to balance a lower budget, but does it serve patients' needs? Integrated computer systems for tracking patients and their care seem like an efficient idea; however, a database is only as good as its information. If information is not continually updated in real time, rather than at the end of a shift or when a computer server automatically backs it up, patients can disappear off the electronic grid, even when they are still bodily present somewhere in the hospital.
} 
Now maybe it's necessary to do that number of people in an afternoon, because I did learn later that they did that number of people in the morning. Maybe it's necessary. But perhaps they could cut it down by one person or two people as a matter of time so they can spend a few moments with them, but then that would have an effect on their efficiency.

This comment echoes Patient G's sentiment that hospital care is "all about money." Subsequent to his angiogram experience, Patient F learns that this particular hospital "does 4000 [heart bypass operations] a year." He calculates: "When you think the number of days, five days a week, for fifty-two weeks, that's really, what, some 250 -some-odd days into four thousand...Of course we got the literature that said they've got the highest success rate (chuckles). Well, I wasn't interested in talking about the success rate." Patient G may be right that, while reducing the perceived efficiency of the assembly line angiogram process might make it more humane for patients, it would also reduce the hospital's surgical efficiency and income. While this hospital's administrators have determined their priority, a review and slight adjustment with reduced focus on fiscal matters might support a slightly slower pace and a more humane communicative approach, increasing the likelihood of ensuring that patients' needs are being as well met as the accountants'. While the number of surgeries, and an accompanying high success rate, speak to the great expertise in this practice area at this hospital, these sorts of statistics, as Patient F suggests, may be of limited interest to an individual patient. An individual patient only wants to know if this particular surgery or procedure will be successful in his or her specific case.

In Patient E's case, surgery is not an option: “....it would've been so extensive they just probably could've cut my head off; that's sort of my kind of way of understanding it. So the 
other options were chemotherapy and radiation." The customized mask helps isolate the

radiation target to "minimize the collateral cell damage." MRI and CT scans were performed at intervals to ascertain the treatment's effectiveness. Patient E describes the movement through the imaging process as "a kind of invisible progress chart," in which patients are moved through each stage of a carefully organized system, from waiting room ${ }^{97}$ to imaging suite. The preliminary step in this progress involves completing a medical history form: ... and one of the things you have to, or I had to, fill out each time I had one of these tests, is how many incredible things could've gone wrong in my body that I tick off. So they want to know; they don't want surprises ("they" being the specialists). So they want to know what experiences have I had medically or surgically? What has been put into my body? What is still there? And it's surreal to realize how

\footnotetext{
${ }^{97}$ In waiting rooms, Patient E occupies himself by people-watching, noting, "I have to wait with other unhappy looking people in a waiting room; and then you wait in a different area, an opportunity to get a look at other ill people." The glumness of the situation is apparent in his description. He is also sympathetic to both other patients and his caregivers:

Especially with something that is as complex and chronic as the ones [patients] that they're [oncologists] seeing. So, this is not like a cold or a broken arm. I don't want to compare the specialties, but this is complex, chronic illness that they're having to deal with, and for many of the people it just keeps getting worse. You can see the fatigue in the doctor and you can see the fatigue in the people too. And at the same time, the doctor who is with me, and he's trying his best, or her best, his/her beeper goes off. And because there's another person with cancer who is imploding somewhere nearby and is in an emergency situation, and at that point I'm not in an emergency situation. And the person that oncologist saw before me, and what was supposed to be a kind of review, had something going wrong with them that made that interview with the patient go forty minutes when it was supposed to be six because there was blood and guts all over the floor for that one. So, for the doctor in a way, or the treater, to remember that about me when, in a sense, the urgency and the acuity of the problem is so great, it's hard. It's a hard expectation. But I'll say it's a good one.
}

Given his experience working in hospital contexts, Patient E is, perhaps, more understanding of the stresses physicians and other personnel face during their shifts. He is clearly able to see both sides of the patient-practitioner dynamic, even when he is in the patient position undergoing extreme treatment. This passage underscores how difficult treatment can be for both patients and physicians, but also suggests that some patients are aware that their physician is under stress and may be late or called away with good cause. Patient E later reflects, “...the staff who have to go home to the spouses or partners who wanted the person that they married, and find they've got a much more tired version of that person. Yeah, I mean it's costly in many different ways." 
many, how much, surgeons and other medical specialists have been able to invent to put into human beings that could create a problem for the imaging, for the technology. So that's also a bit of a surprise. How bad am I? Or boy I'm luck, and how come I'm not so lucky that I don't have cancer, sort of thing?

Patient screening questions are designed to ensure that patients' bodies are safe to undergo imaging; to ensure, for instance, that patients have no metal implants (e.g., orthopaedic, cardiac pacemaker, replacement heart valve, dental, etc.) prior to an MRI. Such forms are requested from patients prior to each imaging session, because health is not fixed and immutable. Patient $\mathrm{E}$ makes the point, however, that certain medical procedures render a body unsafe for certain types of imaging. Patient $\mathrm{E}$ also makes the point that being presented with a detailed list of potential body modifications (these forms also include things like piercings and tattoos, as well as medical implants) is daunting. Patients are suddenly very aware of how much can go wrong with the complex human body, how much medical practice can intervene, and in some way, they may feel lucky. In Patient E's case, the luck feels double-edged: lucky enough not to need any of a variety of implants, but not so lucky to avoid cancer. While these forms are superficially clear, simple, and necessary for safety, it is impossible to know what thoughts go through a patient's head when she or he is presented with such a survey—unless one asks.

Regarding the cancer revealed through diagnostic imaging, Patient E says, "the cancer's not the problem; it's the treatment that's the problem." In an effort to make the treatment more bearable, Patient $\mathrm{E}$ was told he could bring a music $\mathrm{CD}$ to listen to while undergoing radiation therapy or the technicians could play music for him. 
There was something funny that happened. I didn't feel like I ever wanted to bring a $\mathrm{CD}$, but a couple of times they did have one going on, and I just wish I could remember the song. So, the juxtaposition of the song they were playing, and the experience that I was having was so opposite that, again, it was like somebody had a really good ideanot. But it was almost such a really good idea, but it was human then, and the mistake was then, the bad choice of song for me was still a kindness because someone was anxious that I would be uncomfortable in what was a clearly uncomfortable state.

While well-intentioned, this attempt to distract the patient from the activity of radiation treatment, while effective for some patients, may have made this particular patient even more uncomfortable. Patients undergoing this form of therapy are at the mercy of their environment and its practitioners for the duration of each treatment. Again, Patient E's situation speaks to a possible communicative problem, and the need for practitioners to have a conversation about patients' preferences.

Patient B states, however, "I think the doctors are much more open with their patients [than they used to be]. Their patients are much better educated; they do their own research, they do their own work, and if they don't like the doctor, they probe until they get an answer. This makes some of the doctors terribly defensive, particularly the older ones." With regard to imaging, she indicates, "Imaging has so many facets to it; it's part of a much longer train, and your memory doesn't tend to highlight it in the same way as some individual who was responsible for your care. Usually a doctor or a nurse or an incident will override it and your memory doesn't pick up on the imaging." In the medical experience, even when technology is 
involved, it can become invisible to patients; what remains in memory is their treatment by individual staff, ${ }^{98}$ or the diagnostic imaging results.

Several patients voiced positive, if guarded, thoughts about imaging or the medical context. In spite of his traumatic angiogram experience, ultimately, Patient F says, "But I suppose when all is said and done, the procedure is, well it's not simple, but for me it was simple. It was just something I lived through. But the mental aspect of living through it was very difficult." After the fact, he is able to recognize that his strong negative response was entirely emotional; the imaging itself was easy.

The medical images, and their interpreters, had a profound impact on Patient E.

So I mean, I was glad somebody was looking at it so at least I knew that the experience of the MRI, in this case the CAT-Scan, I mean, some radiologist somewhere was going to be looking at it, but more important, it was part of my chart, so it's there to be seen if somebody wants to look at it, and in this case they do want to look at it, and they are looking at it. So I felt a connection. But the other part of it is if the equipment shows that the cancer in my case does not appear to be there, then that's kind of incredible because then I can trust, I do trust,

\footnotetext{
${ }^{98}$ Because patients are aware that some physicians perceive the elderly as time-wasters, Patient A says: I have friends who are just like I am, and if they're seeing a new doctor, they're on their toes. They're ready to sparkle and shine and be so succinct and intelligent and with it, that it's kind of a little thing that you do so they'll become interested in you as human beings, as individuals, and not just an elderly person... [Interviewer: Making sure you're at your very best.] Yes, because I have to be paid attention to. I have to have this doctor. I don't want them to be disinterested when there's something that is, at this time of life, is important and serious. I want to feel that they are like my original doctor, "We're in this together." ... and if I have need to say that I have to tell you something, there's something you need to know. This is what I feel.
}

Here, Patient A's interview makes it clear that it is not only healthcare practitioners who feel stressed when they see an elderly patient; the patients feel it too. Elderly patients may feel the need to do all they can to stand out as unique individuals, rather than just be categorized as one more senior. Younger patients may not experience this sort of agebased stereotyping. 
the people, and I do trust the equipment. And then I can figure out how I'm going to continue to cope with what's been a traumatic experience, but that may not continue to be in the same way it was last year.

Imaging was integral to discovering Patient E's cancer, and then tracking treatment progress. He recognizes, however, that these images require a human interpreter. Patient $\mathrm{E}$ is also aware of the number and diversity of medical staff involved in his care: "but how many people, I mean there are so-he's [radiation oncologist] got students, fellows, there are nurses. I was part of a clinical research trial, so a research nurse. There are so many people who are carers in a complex situation..." Perhaps experience working in hospital contexts, as well as an awareness that his situation could go either way, helped Patient E recognize and appreciate his care. He describes his radiation oncologist in heroic terms:

I view him [radiation oncologist] as a-well this is a time of The Hunger Games. ${ }^{99}$ I never did see the movie, but it's archery apparently, and being a good archer can help you survive in that book. And I view my radiation oncologist as a really good archer, because his task was to shoot me and many, many parts of me, with radiation bows, arrows, in order to kill. And in order to kill as little of me and as much of me as he had to. So, that he really knew science and, in a sense, archery, in this case physics...I was grateful that he cared to that detail.

\footnotetext{
${ }^{99}$ The Hunger Games (2008) is the first of a trilogy of young adult dystopian adventures by Suzanne Collins. It was released as a movie in 2009 starring Jennifer Lawrence as the archer-heroine, Katniss.
} 
Although Patient E admits that the radiation caused a great deal of discomfort, and left permanent physical changes, he remains positive about the treatment. His latest imaging indicates that "the cancer's not there," and given the location of the tumour in his throat, "To even have the voice to say it is kind of lucky to me."

As is apparent in these patients' thoughts regarding the formal channels of medical imaging information, while patients sometimes get the answers they seek, other times, communication is sorely lacking. Some of these communicative problems are with physicians, while others are with technicians or other workers within the medical community. When patients feel that their questions are not adequately answered through formal channels, they turn to informal channels or the media, which may provide less reliable information, and information that may not speak directly to their individual health concerns.

\section{Informal Information Channels}

...and then friends speaking up and saying, "Oh! I'd seen it on TV, but

I didn't like to say that there are class actions going on" [regarding a proposed procedure for this patient].

Patient A

Patients talk among themselves; one can only read so many waiting-room ${ }^{101}$ magazines. Patients often find support and understanding from their peers, people with similar experiences. As

\footnotetext{
${ }^{100}$ While Patient E lists his caregivers, Patient D comments positively on the use of medical teams in hospitals, saying, "They give you a number; it's pretty quick getting the information. I have phoned and I've had to identify myself as the husband or the caregiver, and I have found that it's easier to get the information." Clearly, the recent strategy of employing teams can have a positive effect. The doctor may not be available, but someone on the team, who knows the patient and the situation, will likely be available to answer questions or address concerns.

${ }^{101}$ Unfortunately, sometimes waiting for answers can be discouraging. As Patient A suggests, "I know people who have - younger than I by a good deal — who have ailments, and they're not being, they don't feel any better. You know? So I think they get discouraged, but they keep going, keep trying." This may point to a fundamental truth about patient-practitioner relationships. Patients want to feel better, so they have a vested interested in finding
} 
Patient G suggests, "you talk to other people to see what their experiences are." Given the personal nature of medical intervention, one person's experience may not accurately reflect another's; “...a little bit of knowledge can be dangerous" (Patient G). Patients sometimes share knowledge regarding medical imaging procedures: "I have a friend—she has cancer actually— and she has it [imaging] done down at [hospital], and she said if there's something wrong, they do get in touch with you." While this may be true, the hospital itself might be a better source of information regarding its communication procedures.

Patient F says, "I had no idea what was going to happen, except things I'd heard from other people who'd had angiograms." Again, information regarding what to expect during an angiogram (or any other procedure) should be communicated from medical personnel, either in print or verbally. That patient $\mathrm{F}$ has to rely on other patients' understanding and experiences of angiograms, may have added to his anxiety. While waiting for his angiogram, "you look around at all these other people who are in bed and they're all lying there looking straight up at the ceiling," says Patient F. He suggests that "while you're lying there, you could read over this piece of information. Because that in itself, 'This is what's going to happen. This is why we're doing it.' Really, I had no idea." Rumours and anxiety tend to abound when people feel that they have inadequate information. Reliable medical information explaining the angiogram ${ }^{102}$

solutions, diagnoses, or treatments that will enable them to put their illness in the past so they can re-engage with their normal lives. While doctors also want their patients to feel well, the value of feeling well is greater to the patient than it is to the physician; the physician is not living the patient's experience. While the physician is able to listen, to test, to use his or her comprehensive knowledge to assess and diagnosis, sometimes, even after all these steps, the physician is still unable to satisfy the patient, to help them return to their normal life. As a society, we perhaps expect too much of the medical profession. Regardless of our doctors' best efforts, eventually we all age, may become ill with something for which there is no cure, and die. While some patients doggedly fight this inevitable trend, others simply give up. Patient A continues, "I've certainly heard that people, for instance, aren't continuing going to the dentist because they're older. Why bother? That creeps in. They're busy accepting the fact that they're going to die and they've got enough to work on, you know? Perhaps."

${ }^{102}$ After his traumatic angiogram experience, followed by quintuple bypass surgery, as previously noted, Patient $\mathrm{F}$ finds few opportunities to work through his experience. Although he attends a post-surgical support group, opportunities for one-on-one discussion might have been more helpful for him, and probably other patients too. The book he is given to help him and his family through recovery includes a section on the emotional impact of heart 
procedure, provided to Patient $\mathrm{F}$ at any time prior to the procedure, might have decreased his anxiety.

Patient D conjures other people to help rationalize his claustrophobic MRI experience: “...it's used by thousands of people daily around Canada. I've never heard of somebody not coming out.” The MRI experience causes Patient E to wonder, however, “there's this big equipment, and it's big enough that the staff leave the room, you know? Hmm, this is not a place where any other human being wants to be. I wonder why?" He knows, of course, but being left alone in a room of radiation-emitting equipment creates an uncomfortable sense of confronting a dangerous situation alone.

Hearing both positive and negative reports from friends who had experienced internal scopes, Patient A is primed for her first experience. The patient is placed in a vulnerable position for the scope to be introduced. Patient A's reaction to her scope is unexpected: "Well, it was funny, you know, the position you're in is so funny. So I started to laugh, and the two nurses were extremely, you know, they played into this and so the whole thing was very light and relaxed." Because she is able to see the ridiculousness of her position, or perhaps as a coping mechanism in a potentially embarrassing situation, Patient A is able to laugh; her laughter proves infectious, and lightens the experience for everybody in the room. Her experience proves to be quite different from those of her friends. ${ }^{103}$

surgery. Although the book states that, in Patient F's words, "It's possible the patient's emotions will be very, very different," it is not until a friend, who has also undergone bypass surgery, phones and asks, "Have you cried today?" that Patient $\mathrm{F}$ feels that he has permission to cry. As he says, "I remember thinking, why me, etcetera. But he [the friend] was the first person who acknowledged that that happens, and did I ever breathe a sigh of relief when he said it." While print resources and support groups may be helpful, they may be inadequate. Referring to the print resources, Patient F says, “...for some reasons, all they say is that there will be emotional stresses. They don't say, 'He will cry,' which I think they should." Speaking openly with someone who has similar lived experience, especially a close and trusted friend, was necessary to allow this patient to let go of the emotional stress he carried before, during, and after surgery.

${ }^{103}$ Patients E and F both comment on the successes and shortcomings of support groups. Some hospitals have established group therapy sessions enabling patients to find support from people with similar health issues. As these 
groups are administered by the hospital, information can be filtered back to medical staff, and issues that may arise can be readily clarified. Patient F mentions that prior to his cardiac bypass, he was in a group of half-a-dozen or so patients all about to undergo the same surgery. The pre-surgery information session was fine; however, a different dynamic was apparent in the post-surgical meeting:

On one day I had to go back for my follow-up, and those five people were also there, and one woman was having a terrible experience. And as a result-I remember now, although I didn't think much of it at the time - we all moved away from her. We wouldn't have, literally, we wouldn't have anything to do with her. We didn't sit with her and chat. So when I think that, I'm dealing with my own stuff. That must make the type of work you're trying to do that much more difficult because you can't get a group of people telling you. It's got to be each person alone. Patient F's observation, that the group reacts by isolating an unwell member, is not something that had occurred to this researcher. Notions of group interviews were dismissed due to logistical and privacy concerns. Patient F reflects that when patients are unwell, or recovering from life-altering surgery, it becomes more difficult to be sympathetic to other patients; one is somewhat trapped inside one's own head, trying to unpack the events of one's own body. Drawing on Frank, one might be said to be figuring out the narrative details.

Patient E draws on both his experience in cancer treatment group therapy, as well as his professional experience as a social worker in hospital and group-work settings. He is uniquely qualified to make his observations: Well, and not a lot of interest in talking about getting support. Women, and here I have seen over a career, who have illness and, say cancer, are much more ready to have conversations about it; to seek help, and to seek support. So there's an assumption, I think, that the healthcare community makes that women are more amenable to it. I think men are probably too, but I think because the man starts by saying, "I don't want any person to help me. It's bullshit. It's touchy-feely. I don't want any of this." I think because that's the starting point, and it probably is for many people, but more men than women, that's where the physician, I won't say the doctors, but that's where the health professionals stop. It needs a little more work to peel the onion a little bit more. "Yeah, I hear you saying this is bullshit, and can we talk a little bit more about it?" Because then I think just like in prostate cancer there, I mean again, I'm looking in the past decade, there are lots of support groups. They function different than a woman's support group. A women's group, and here I speak from my experience with the Cancer Society, a women's group, say breast cancer, will be much more ready to talk about one another's experience, and much less needy of structuring a meeting. A men's group generally will say, "All right, we need a chairman, or we need an agenda. We need an agenda to know-we need speakers." So, even though it's a support group in both cases, the men seem to have to replicate perhaps many of their work experiences in their support group. And the women are smarter than that. So, in that way there is a difference, and I expect the health professionals, when it comes to the one-on-one relationships with the people with the cancer experience, that there'd be even a more-women may get a better chance from the healthy professionals without it being conscious, that they might be wanting to talk, or might be interested in support. The men, if they're kind of tough at the front end, you know, "Beat this...," which, I don't know, I guess my fantasy hard drinkin', hard smokin' guy, has to all of a sudden be vulnerable? But they probably are. Different notes that have to get played. And if they've had a lifetime living with someone who's never seen them do that, or with children who've never seen their dad behave in that way, it may not be something they want to start doing. But it's an opportunity.

While it may not be obvious that support groups function differently along gender lines, Patient E makes a fascinating observation. Reflecting on some male patients' earlier comments about the need to feel macho, even in the medical context, it is not surprising that at least some men find group session work difficult or impossible. Patient F comments, "But I think, other than the family, you're the only other person I've ever talked [to] about the things as I did today." The tendency for male patients to suppress emotional responses in obviously challenging medical situations is an unexpected finding. It also raises the question, how does suppressing intense emotion impact health and recovery? Whether this tendency for suppression is generational, remains to be seen. 
A stereotype may exist that patients tend to be very self-focused and self-centred. Patients can also be concerned about the translation of their experiences to other patients, as well as the stresses on their caregivers; they can be surprisingly outward looking and sympathetic. Patient B, probably the most self-absorbed interview participant, expresses concern about patients not receiving enough diagnostic information to enable support:

You know, if you ask somebody, you know, they're telling you about a visit to a doctor and you say, "Well, did you ask him that?" "No, we didn't even talk about it." You think about the person and you think, well, and she may not want to know. Some people are more comfortable not knowing, leaving all the responsibility somewhere else, but I mean, if a woman does that and her husband says, "Well, what's going on?" she's not going to have his support either, because he's going to be feeling in a very lonely and unsustainable position. I think the doctor needs to think a little bit about a patient, how much they really want to know. And if they ask aggressive questions, I think, you know, that they really want straight answers. If they ask timid questions, you need to be a little more careful, be more circumspect about what you say.

This passage points to the challenges for physicians of patient-centred interviewing. Ideally, a doctor tailors his or her communicative style to the needs of each individual patient, considering the patient's verbal, as well as non-verbal, cues. This kind of communicative work requires a great deal of attention, leading some physicians to dismiss it as too time-consuming for their practice. Once a diagnosis has been revealed, asking the question, "How much information 
would you like?", for instance, may only take a few seconds, and can often be answered with printed material or reference to other resources. The advantages to the patient, as given in Patient B's example above, can be enormous, helping the patient and her family understand the medical situation, but also helping her find the necessary emotional support at a vulnerable time.

As well as medical staff and other patients, people also rely on family and friends for information, as well as emotional support, through difficult health situations. Patient A says, "Thank goodness I have other support. Some people would not have that." As she expresses, having support beyond the medical environment has proven very helpful:

I have daughters who keep informed. I have friends who keep informed, or have information for me, and that's certainly wonderful to be, to have people around who are, who care, and have that information. So, they're constantly finding things that will help. So you're not dependent on the doctor only, and I would think that in my situation where my doctor was there one day a week, you've got to have other...

The additional information, as well as emotional support, has sometimes helped her understand her medical situation more clearly, or make better informed treatment decisions.

Both Patients D and E discuss the importance of having their wives present during medical appointments. Patient D considers himself "more fortunate than my wife" in terms of health. He acts as her caregiver, and routinely accompanies her to medical appointments, often sitting with the physician or technician:

It gives us an opportunity of finding out what the patient hears and what the observer hears, and do they match. And I have found on a 
couple occasions that there was a difference in interpretation. And I have found that the observer probably is closer to hearing the fact, and my wife and I will now not go without the other.... doctors know the patient, if it is my wife, wants me to be with her so we both can hear, and we both can go home and talk about what we think we heard, and do we need further clarification. And in a couple of cases we did.

Similarly, Patient E expresses:

...for most of the appointments I came with a notebook because we both listen well, but she [my wife] actually wants to remember it. And, again, it's an opportunity to take some of those words and get a translation. And she understands me well enough to know that sometimes, though I'm listening, I may not be hearing what they're saying, or I'm all ready to go to the next topic on my mind. So we have a really great joining of-it's really helpful. That's really helpful because there is so much. There is so much. And there are so many different health professionals who come into your life.

Patients are not always the best listeners. Especially when receiving a serious diagnosis, the patient is mired in his or her own health situation, which may be frightening, or they may not feel well; such factors impact the patient's capacity to listen and/or understand the information being offered. Whether a spousal partner, other family member, or close friend, as both Patients D and E express, it is advisable to bring an advocate to medical appointments around serious health issues, regardless of one's age. Having someone present to listen and take notes, helps ensure that questions of concern that the patient has expressed prior to the appointment, are heard, 
answered, and that the answers are understood. Not all patients have a spousal partner or family member able to attend their medical appointments. In the US, it is now possible to train as a patient advocate, ${ }^{104}$ accompanying patients, helping them navigate the complexities of the medical system, and ensuring that their needs and wants are met. As Canada's population ages, and hospitals continue to try to do more with less, trained patient advocates may become more common.

This section has examined the pros and cons of patients receiving imaging information through informal channels, such as other patients, family, and friends. When encountering a new experience, a common human reaction is to try to understand what that experience will be like. If inadequate information is provided by healthcare professionals, the chance of patients seeking information elsewhere increases. While unsanctioned information is usually provided as a caring response, the quality of this information is inconsistent. Furthermore, some things we cannot know until we ourselves experience them; every patient is a unique individual.

\section{Media Information}

And then the cardio inversion... I said, "What's that?" And she said, "Well, we shock your heart.” I said, "With two paddles?” She said, “Oh, yeah." I thought to myself, my God I've seen this on TV...

I looked back and there was a nurse standing there. I said, "When are they going to do it?" She said, "It's done."...Now if they had told me

\footnotetext{
${ }^{104}$ For instance, Sonoma University in California offers a certificate in Health Navigation; the University of Toledo offers an online graduate certificate in patient advocacy; and the University of Miami offers the online Alfus Healthcare Advocacy Program. These tend to be short online courses, specific to the American healthcare system. No Canadian equivalents were located; however, patient advocacy groups have formed. Patients Canada encourages patients to tell their stories online; their website also includes a section on Patient Advisers. In 2007, Open Arms: Patient Advocacy Society formed in Alberta, and is available to assist patients and their families navigating difficult medical journeys.
} 
that. Again I was - the idea of these paddles, what you see on TV, the body jumps six feet.

Patient F

As well as personal contacts, older adults also engage with media information: radio, television, newspapers, and the Internet. In the interviews, this source of information is often just a vague allusion: “...part of the worry is just caused through reading newspapers, what you hear on TV and you become suspicious...it's one of those things that we read and hear so much about; diseases and in particular things such as cancer...” (Patient D). Patient B echoes this sentiment: "There's so much discussion available in the media in one way, shape, or form - and even the timid people read the papers." When asked if he considers improved patient access to medical information positive or negative, Patient D responds: "Well, I'm going to have to answer both ways. It's good that we can find out a lot quicker. We can find out what options there are; however, having said that, one must be careful as to what they look up, what source they're getting it from and actually how they read it." Patient B agrees, saying: "I probably read three, four, five [online articles]... I'm kind of fussy about not getting carried away on one person's say-so. You know, some of the stuff I look at, that's just a commercial venture, not a research venture." A lifetime of media engagement, perhaps coupled with growing cynicism, has made at least some seniors aware of the need to engage with media critically, an ability they translate to newer digital media like the Internet. Patient D suggests that access to more medical information has both negative and positive effects:

...it's easy to jump to conclusions and [self-]diagnose and diagnose the wrong way...But that I can get to something quickly and say wow, maybe I better go and tell my doctor. I don't know if what I saw is 
right or accurate, but it's enough reason that I'll just phone, and if he wants to phone me at home and say yeah, come on in, or don't be silly.

While a little knowledge may be a dangerous thing, an advantage of increased access to medical information for patients is that it may alert them to potential problems, allowing them to seek appropriate medical help sooner, rather than later.

In some cases, patient vigilance is a good thing; however, Patient $\mathrm{C}$ suggests, "this [patient access to medical information via the Internet] has been probably becoming a real problem for doctors," in that patients sometimes overwhelm healthcare providers with irrelevant information and unfounded concerns. Patient B suggests that her search for medical information begins and ends with the Internet:

...if I didn't get an answer, or didn't think to ask a question, I could get the answer on the Internet...I mean, I'm prepared to ask questions if I've got a suspicion, and ask questions means I go to Google. Or if I don't, I go to my doctor, but Google is easier...It's much more immediate.

Although Patient A acknowledges that she could seek information online, she declares, "I don't go where I feel I don't need to go.” Depending on the individual patient, how the information is used, and the reliability of its source, increased access to medical information may be more or less desirable, positive or negative.

Increasingly, hospitals, and other medical institutions, are adding communication technologies to their operations, both administratively and for patient education and blog 
conversation. As part of his patient education, Patient $\mathrm{E}$ was given a video prior to his treatment. While he thought it was "pretty good," he qualifies this review:

Pretty good in the sense that there's a lot of content in it and it gives you some images, and I learn visually and I don't mind that. But again, because it's so early in my career as a person with cancer, I don't know how much of me is available to take in all that stuff. ...And I don't know whether my story is the same as the person in the video tape. It doesn't seem to be. So a lot of it's there, but a lot of it I think just goes by.

Needs regarding the quantity of information, and when and how it is delivered during medical treatment, are unique to each individual. Some patients may be satisfied with relinquishing control to physicians, while others may wish to be well informed and actively involved in treatment decisions. Some may be satisfied retrieving information from digital sources, videos, or pamphlets; others prefer opportunities for discussion, supporting clarification and a deeper understanding of their diagnosis, treatment options, and prognosis. To provide truly ethical patient care, physicians must be cognisant that patient communication is not a one-size-fits-all proposition; patients are unique individuals, so effort is required to understand each patient's communicative needs.

\section{Patients' Reflections and Rationalizations on Imaging}

...if I can see a picture while I'm listening, that'll get a-and then I can think about it—-then that's all good. So, if somebody asks me those [questions], if someone's aware of that, that there may be ways 
that I put it together, this picture, that is different from the way that you do...

\section{Patient E}

Patients often reflect on their imaging and medical encounters after the fact, developing their own ideas about their experiences, developing their own stories that become part of their continuing lives. Given the difficulties in finding appropriate words to discuss imaging experiences, it is interesting to note the language that Patient E uses. Regarding a CT scan, he says: “...it's an image of me that's different from the one I look at when I'm in the mirror. It's exactly what I'm looking at when I'm in the mirror, except I can't see it." As cited at the beginning of Chapter 4, the notion of the medical image as a mirror is sometimes used in academic discussions (Radstake 116; Wall 139).

Later, Patient E says, “...if it was a photograph somebody took, they might say, 'Here’s the photograph—-headshot.' Because this was my headshot..." As an occasional actor, the language of the headshot, a promotional photograph of the actor, seems an obvious way for Patient E to relate to these images. He also uses the possessive; this image is "my headshot," alluding, perhaps, to the sense of ownership to which Blaxter refers (CVP 771). Ultimately, language may be too dull a tool to fully discuss the complex relationship between patients and their images. As more of our lives merge with the digital, the language to distinguish between the real and the imaged may become more blurred and illusive-perhaps even irrelevant.

Pointing to a potential generational difference in relating to imaging media, reflecting on his angiogram experience, Patient F suggests, "I suppose if I'd looked at the monitor I might have reacted differently, but I wouldn't do that (chuckles). Sheesh.” While somewhat selfdeprecating, this statement suggests that there may be a difference in the experience of monitor 
viewing for individuals who gained life experience prior to digital technologies; perhaps the response of a younger demographic would be more receptive. Younger patients, who know no world without advanced digital interfaces, might view the screened image as strangely comforting, or pleasantly distancing, creating the detachment or self-objectification alluded to by Wall $(142,139)$ or the "distributed embodiment" discussed by Radstake (129). While monitors may be comforting for some, imaging machinery remains problematic.

Patient D finds the MRI terribly claustrophobic, suggesting, "I think if they put me in feet first I would've felt better. But they don't, you go head first, and it just seemed like a tunnel that wouldn't end." Upon reflection, however, he says, "it's no big deal." When asked for clarification, he finds "just going into the equipment" was the terrifying part; but "I'm not sure I was even thinking of the results at that point. I was just thinking of the experience of being in the MRI machine or whatever it is. But after the fact, and to this day I still think of it." These statements reveal somewhat mixed messages. Clearly the MRI experience caused an anxious claustrophobic reaction; "it's no big deal" are the words of a survivor, someone who has come through a difficult life event and is still here to talk about it, an attempt to rationalize a traumatizing experience and minimize its discomfort. Patient $\mathrm{D}$ ends this part of the interview saying, "It's just I've often thought I hope I don't have to have another MRI." Clearly, even if "it's no big deal," the MRI experience is not something Patient D ever wants to repeat. Complex, or conflicting, responses are apparent in other interviews as well.

For instance, Patient $\mathrm{E}$ reflects on his identification as a person with cancer, which is primarily based on diagnostic imaging.

I think when I was trying to manage this notion of having been a person with cancer, there's so much—it's such an overwhelming label 
to have, especially in my situation, because I really wasn't feeling ill.

I had really very little indication that anything was wrong. So, all of a sudden I'm given a diagnosis that requires me to be - that alters my life, perhaps terminates it.

The patient impact of diagnostic medical imaging is summed up in this short passage. When a patient is feeling generally well, to receive a diagnosis of stage IV cancer is shocking, instantly shifting one's self-perception from that of a healthy person, to that of someone whose death may be imminent. Patients in this situation are forced to trust the imaging technology and image interpretation; they must develop strong faith in the treating physicians. This is an extremely disempowering position, as will be discussed in the next chapter. Patient E continues: The shock of the diagnosis, the rapidity of the need for treatment and the fear about the treatment is - in my case radiation and chemotherapy — are bigger than the worry about the technology. So I view the technology as something in between me and, in my case, the radiation oncologist and the medical oncologist. So I saw them [the imaging technologies] as necessary, a bit alien, and get on with it because they get me closer to the start of the treatment. I was told, and this is probably important from my experience, I was told that with this cancer that I have—-had — the likelihood after treatment for a good prognosis was pretty good; so it was curable.

In his case, the technology effectively becomes invisible. Patient $\mathrm{E}$, who is suddenly thrust into a life-threatening situation, is more focussed on treatment and recovery, than on anything mediating those activities. This is a unique experience from any of the other interviewed patients, 
for whom the imaging technology is frightening. It may be worth observing that Patient $\mathrm{E}$ is also the youngest interview participant, at age sixty-nine, and until this point, a generally healthy person, so perhaps more confident in his ability to engage with medical technologies. Further reflecting on the imaging technology, Patient E says:

Well, it's comforting to know that the science is going to inform the practice, and that the practice is designed to be helpful... I'm not terribly interested in technology, so how marvelous it is that there's this huge machine that will help me is, I mean I'm grateful to the scientists who created it, but I'm not really interested in how it works, and so it's a matter of enduring it, and that, in that sense alien—alien to me in that it's not part of my immediate experience that I wanted to know about. And it still doesn't really interest me, the science. But as a result of having seen some images, I'm blown away by the information it provides. So it was alien and it continues to be alien. The more often I have to go into these rooms the less odd it is, but it's never comfortable. And I like to be comfortable.

Patient $\mathrm{E}$ decides that the most appropriate word to describe the imaging machines, MRI or CT scanners, is "alien." While unique to this patient, this word is used by the participant half-adozen times during his interview. Patient E's consistent use of the word "alien" relates to Blaxter's use of "alienating" to describe imaging technologies: "they are often described as alienating to patients - little understood, perhaps frightening, the means by which illness is turned into disease. Technological representations hide the selves embedded in human bodies, reinforcing the already alienating effect of hospital medicine" (CVP 763). Patient E also speaks 
of "enduring" imaging sessions. Intellectually, he appreciates that imaging helps assess treatment progress; however, undergoing imaging is taxing, and it takes effort.

Although most of Patient E's encounters with radiation are for treatment, rather than imaging, he speaks about this experience a great deal during the interview. His treatment is a direct result of diagnostic imaging. Given the concerns about radiation discussed in Chapter 2, it is potentially frightening that radiation is used in cancer treatment. Although effective for destroying cancerous cells by burning them, radiation also damages healthy tissues and causes deleterious effects for the patient. Reflecting on this part of his experience, Patient E states:

What's interesting to me in terms of the technology, and the radiation piece of it, is that because the treatment for throat cancer is so, well, for me it was very aggressive. But it's so primitive that you can't, I mean it's-you can't swallow, you can't eat... And then the radiation has a kind of half-life. In the first couple of weeks it's really no big deal. I mean, first of all the radiation isn't touching you, so it's like the dentist experience... And then at the same time with the kind of radiation experience that I had, it's cumulative so as the radiation is in you, it stays in you and then it's prolonged, so you get worse and worse. When I got worse and worse I lost weight, which is not surprising... I know it's unsafe for me to be in here [having radiation treatment], but it's like, I wonder when I leave the hospital now and I'm full of radiation, or I wonder what the — does this stuff, am I radiating stuff to you? And I'm sitting here, so I asked them these kinds of questions because I don't know. Who would know? You 
wouldn't think to ask that stuff. You know when you've got thirtyfive days of radiation in you, and you're going to get souvenirs like this [touching the dewlap at his throat], no taste, a dry mouth. Okay, so where else it this? It's pretty—it's why they leave the room... Well, I think for me, I have asked and that [radiation seeping from the patient] isn't happening.

In the post-atomic age, given the number of popular culture references to the adverse effects of radiation, our culture tends to be concerned about obvious radiation exposure. Given this context, it seems surprising that Patient E actually has to ask whether his body may be seeping posttreatment radiation, and that he does not recall this information being offered in the patient literature or pre-treatment video. According to the website for the American Society of Clinical Oncology (ASCO), in this type of external radiation exposure "the patient does not become radioactive; the radiation remains in the treatment room;" however, "because internal radiation therapy causes the patient to emit radiation, a number of safety measures are necessary" (ASCO). Internal radiation uses radioactive "seeds" or other physical delivery methods implanted in the patient's body to introduce high-dose radiation targeted for certain cancer types. In such cases, patient visitors must stay "at least six feet" from the patient, visiting for a maximum of half-anhour per day; children and pregnant women should minimize or avoid exposure to the patient for two months (ASCO). Although Patient E's treatment was "beam" radiation, it was still very aggressive; Patient E declares:

Well I felt like, well yes, the cancer cells were being killed. That was the reason they were filling me with chemotherapy, sort of from my bald head to the tip of my toes, which was like a shotgun that was 
going to, theoretically—no not theoretically, in practise — visit as many cancer cells and kill them. That was how I understood the reason for this quite dreadful treatment. And so that's a killing that I felt, and I felt, with that treatment that I was being killed. I really felt, as I shared with you, by the second treatment I was pretty close to as dead. And then I wasn't in a movie I was all- just above being dead from the treatment. If this helps, even with a kind, caring empathic medical oncologist, it doesn't feel like it. With the radiation oncology, you're revisiting - you get on a table, you're getting anchored onto the table, locked onto it with or without music. You're getting, these cells are getting radiated. And the cells in front of them, which are healthy, are getting killed — and I would use that language — in order to get to the cancer cell which he wanted to kill. [Interviewer: So there's sort of collateral damage?] Oh yeah. And so, for me, after a while I'm getting the dry mouth. I have this sort of mass that sort of hangs. It's a dewlap is what they call it. You know, it's smaller than it was, but you know in a turtle neck sweater it's like this is a huge souvenir of radiation because this is the mass that gets - that I have and will have. And in a way I view that as kind of, not my Red Badge of Courage, but it is kind of like, I don't mind wearing this.

Patient E feels fine when he starts treatment. The treatment itself is enormously damaging to his body, leaving him with permanent physical changes. Cancer campaigns often use the 
violent language of the fight, the battle, to communicate treatment to the public and to patients. When asked about this rhetoric, Patient E responds:

But I don't view the cancer, I don't see that as a fight, because I'm not sure for me that I'm free from cancer because I know, I'm aware that cancer can visit me again in different parts, and very well may. But it hasn't visited me in my throat yet, and that's what I've just learned from the scans and that's all good. So, I don't feel that cancer is a fight because I don't even view it as an enemy. I just see it as, you know, it's an illness that can happen, and it can happen to many people as they get older and even when they're young. And it can happen in many sites, and it can spread. And I got lucky in 2011, 2012 - unlucky-lucky. And that can continue. So, I don't view it as-I don't actually like-I think it's the treatment that is more of a killing experience than the cancer. Certainly that would be how I would characterize it. But no, the war imagery and the survivorship, because I'm not even sure that, because again I did, I survived. Certainly this week I can say to you that from the scoping there doesn't seem to be any indication of cancer in the throat...

Just as the imaging initially identifies the cancer, imaging also allows Patient $\mathrm{E}$ to declare, at least for now, that he is cancer free. Images, and their interpretation, hold enormous power over patients and their sense of wellness. Reflecting on the news that the imaging shows the cancer is gone, Patient E says: 
The meaning, I think what I-it gets real primitive. The reason why I'm having all these tests is because I'm a person with cancer. That's time one. Then time two is, “Am I still a person with cancer? I know I'm still a person." And then if the results of the test is you're no longer a person with cancer, that's when the listening, that's sort of message received...And okay, translates, is the cancer where you want it to be? Is it in the garbage can?

Patient E's state of being is informed by diagnostic information; through imaging, his identity becomes linked with the cancer. As imaging provides information that the treatment is successful, he can return to being "a person," rather than "a person with cancer."

Patient E also acknowledges the issue of cost - something many of us in the fortunate position of having provincially funded health plans may not consider. The popular notion is that provincial plans provide "free" healthcare, but in fact, we pay for such plans through taxation. Information about how much our care costs through a provincial plan is not transparent. Patient E suggests,

The one thing that I don't know is how much it costs for those treatments, and I'm not sure that it wouldn't be-I'm not sure I'd want to be charged, but I rather expect that my cancer experience has cost a pile of money. I mean, we know it has, but I don't know that. And, I mean, if I were in the States, I probably would know how many hundreds of thousands of dollars of care, whether I was insured or not, and assuming I was, that it would cost. And I think it might help me, I mean it helps me to know, is that this experience of illness 
has not bankrupted us. I mean it's incredible to be in a society where, as a citizen, I've been able to have excellent care, and with a good outcome, without really having to reach into my pocket very much at all over time, and as a tax-payer for sure paying... And I think for this technology, because I know it's so expensive, and I know it breaks, and I know it also works, probably wouldn't hurt me to know what my community is paying so that I can have what is basically — it seems free, but is actually very costly care to make me better. That [knowing the cost] wouldn't hurt. But I also think that it would help me to know the next time I went for a CAT-Scan, and I will, or an MRI, that roughly speaking, this cost X-thousand dollars.

His point is that perhaps we would better appreciate our "free" healthcare if we had a clearer understanding of what it actually costs to receive treatment. Patient E continues:

...this is very costly to treat ill people, and it's very costly to treat people who have illnesses that keep getting worse, or that need to be monitored much. So, I could go the rest of my life without ever knowing what is a ballpark figure for a CAT-Scan cost? But I could also know and sort of have some sense of, okay the doctors are complaining about how much they're not getting paid, or paid, and the province is complaining about the billions of dollars for healthcare. But what do these, how much do these, what does this represent? ... at the end of a year-and-a-half of this kind of experience, since we're talking about it, and it's valued and valuable, I don't really 
know. It's not political. I guess the point I'm trying to make is that I don't raise this with a view toward the politics of healthcare, but I do think it's useful to know how many, how much is a hospital bed? Not to buy a bed, but if someone's staying in a hospital overnight? What is that? And in most hospitals I can probably say $\$ 800$. Okay, so if I stayed in hospital for say four nights, it’s $\$ 3200$. Wow. And again, it helps me understand why there may be a pothole in the road. And if you ask me, well, "Would you rather not be admitted?" No, no that's not why I'm asking those questions.

In Ontario, information regarding healthcare costs is not readily available to the public; the Ontario Health Insurance Plan (OHIP) website does not offer such details. The OHIP website does, however, post its "Interprovincial Out-Patient Rates" (April 1, 2014). These are the rates OHIP charges another province's health plan, if one of their patients requires treatment in Ontario. In this case, OHIP charges $\$ 630$ for a CT scan, and $\$ 686$ for an MRI "including radiologist services" (OHIP). ${ }^{105}$ One can surmise that these rates are similar to the costs that Ontario's hospitals charge OHIP for these imaging types in our "free" healthcare system.

As Patient G comments, "Yeah, I would say I think we're very fortunate in this country to have, in this province, to have the medical system that we do have, and I wouldn't want to change it except there's a lot of room for improvement, and a lot of money's going into the system." This patient, and the others interviewed, experienced Ontario's health services before

\footnotetext{
${ }^{105}$ Private clinics in Canada also offer both CT and MRI imaging, and advertise their price lists. Canada Diagnostics, with private clinics in BC, Albert, and Quebec, charge $\$ 600$ for a non-contrast CT scan, and $\$ 1100$ for a contrast CT scan; MRIs range from $\$ 900$ for straight imaging, and up to $\$ 1600$ with contrast. Ultrasounds are available for a flat rate of $\$ 500$ (Canada Diagnostics). With services in Gatineau, Quebec, St. Joseph MRI's rates range from $\$ 745$ for a straight MRI, to $\$ 1255$ for more complex imaging, or imaging requiring contrast (St. Joseph MRI).
} 
OHIP; provincially funded hospital services came into effect in 1959, while physician service funding began in 1966 (Ontario Ministry of Health and Long-Term Care). The provincial government estimates that OHIP will cost $\$ 13,806,754,000$ in the $2014-15$ fiscal year, an increase over the estimated budget of $\$ 13,378,813,300$ in $2013-14$, which was an increase over the actual 2012-13 budget of $\$ 13,278,213,387$ (Ontario Ministry of Finance). Both Patients G and E recognize that our healthcare system is anything but "free." As the province and Canada see a demographic increase in older patients, frequently with multiple co-morbidities, these costs will continue to escalate.

In spite of the costs, Patient B suggests, "I think that we ought to have better facilities for imaging, and distinctly more. ...The availability is questionable." Smaller centres cannot afford scanners; consequently, their patients are transported to larger centres if they require scanning. This puts additional stress on both rural patients requiring imaging, and the facilities in larger centres. The availability of imaging technologies is questionable, but as discussed in Chapter 2, a high percentage of scans may not be warranted or helpful. Availability might be improved if physicians were more selective about using this equipment, and if patients were less insistent on demanding its unwarranted use.

Reflecting on the contemporary use of medical imaging, Patient A suggests, "Well, thank goodness they have these images now, because at one time they didn't, and now they know so much more." In terms of patient care, imaging provides more information, and with greater detail, than could have been gathered using earlier medical technologies. The question of care revolves around how the images are used, and how their interpretation is communicated to patients. Reflecting on a serious post-surgical incident, Patient A comments: 
...lessons learned, and an ability now to see more sides. More and more sides as you get older, hopefully that change where one won't get stuck but continue to see all the possibilities that exist in other people's minds. And I think that makes you maybe stay younger, or alert generally if you're able to see more...So that even if one is older...you've got years of hearing, and seeing, and experiencing. And if you put them together it will be useful-hopefully. And that's what, you know, if you just look at someone as an elderly person, you don't see. You really have to be in conversation.

This closing again supports the importance of patients in medical dialogue. Regardless of their demographic, patients bring their own experiences into the medical discourse; these can shade or enlighten their perceptions of healthcare personnel and situations, as well as imaging and other medical technologies.

\section{Conclusion}

This chapter has analyzed information from the interviewed older patients regarding communication through various channels, and their thoughts and rationalizations regarding medical imaging. Regarding formal channels of medical imaging information, while patients sometimes get the answers they seek, other times, communication is lacking. Some of these communicative problems are with physicians, while others are with technicians or other healthcare workers. Older patients may feel included or excluded, informed or ignored; physicians and other healthcare workers are sometimes perceived as making assumptions about what patients want to know, and how they want to know it. While the imaging technology itself 
might be perceived as neutral, the ways in which it is used, the ways in which it mediates patientphysician discourse, can support or impede patient understanding, acceptance, and reaction. When patients feel that their questions are not adequately answered through formal channels, they turn to informal channels (other patients, family, or friends) or the media, which may provide less reliable information, or information that does not speak directly to their individual health concerns. Many are, however, very media savvy and critical of the sources and quality of their health information. Frequently, older patients find themselves lost in a complex and unfamiliar system; consequently, older patients feel the need to educate and advocate for themselves, and/or be accompanied during healthcare visits.

Older patients are conscious of costs, generally feel very lucky to live in a context that pays for their healthcare needs, and are often sympathetic to overworked physicians. They are also, however, conscious of fiscal waste in the medical system, often around administrative tasks. Although patients may struggle to comprehend their images, both medically and philosophically, they are grateful for access to imaging, feeling that it provides reassurance regarding their health condition. Some are also concerned with imaging costs, and sometimes unhappy with a lack of communication or information made available to them before, during, or after imaging procedures. Although imaging technologies can evoke strong negative emotions, it can also become invisible when detecting or treating serious health conditions, when the focus shifts to more personal mortality issues.

The discussion of patients' responses to power and control, a Foucauldian discourse analysis, appears in the following chapter. 


\section{Chapter 6: Older Patients' Responses to Medical Power}

But the word power is apt to lead to a number of misunderstandings....

By power, I do not mean "Power" as a group of institutions and

mechanisms that ensure the subservience of the citizens of a given

state....It seems to me that power must be understood in the first

instance as the multiplicity of force relations immanent in the sphere

in which they operate and which constitute their own organization; as

the process which, through ceaseless struggles and confrontations,

transforms, strengthens, or reverses them; as the support which these

force relations find in one another, thus forming a chain or a system, or on the contrary, the disjunctions and contradictions which isolate them from one another; and lastly, as the strategies in which they take effect, whose general design or institutional crystallization is embodied in the state apparatus, in the formulation of the law, in the various social hegemonies.

Foucault, The History of Sexuality 92-93

Continuing the analysis of older patient interviews from the previous chapters, and using the same methodology, this chapter explores the question: Does the patient respond to notions of power or control? How? The patient-practitioner relationship around imaging is examined using a Foucauldian lens to focus on notions of power. The most obvious sovereigns of medical power are physicians and other healthcare workers, and a physician-dominated power struture is apparent in some patients' remarks. For instance, Patient C states, "Somebody told me that I had to have the thing [imaging]," or Patient D says, "my family physician thought it was worth 
checking out, so they sent me [to the hospital for an MRI]." "They," meaning physicians and other healthcare workers, are mentioned a great deal in the interviews, and often "they," seemingly invested in a reductive medical gaze, have the power to determine what actions are taken on a particular patient's body. True to Foucauldian conceptions of power, however, power can shift and power struggles may not be as obvious as they first seem. Surprisingly, sometimes the imaging technology, or the images themselves, seem to hold power over both patients and physicians or other healthcare workers. While patients' power may seem limited, some interview participants found ways to assert their own power in these vulnerable situations, sometimes in surprising or subtle ways. Discovering that patients can assert themselves, that they are more than "docile bodies" in a disempowering context, lends credibility to the notion that medical knowledge can indeed be coproduced by patients, physicians, and available technologies (Foucault DP 135).

\section{Physicians' Power}

We should admit... that power produces knowledge...; that power and knowledge directly imply one another; that there is no power relation without the correlative constitution of a field of knowledge, nor any knowledge that does not presuppose and constitute at the same time power relations.

Foucault, Discipline and Punish 27

Well, I was brought up at the tail-end of the era where, I mean, you believed fully what your priest or your minister said, what your doctor said, what your teacher said, what the policeman said. So, a lot of it 
you just wouldn't question. You took it for being the truth rather than asking, is there any other information that may be hanging round the outside edges that is likewise important?

\section{Patient D}

Although the lump Patient D's physician finds and scans is determined to be benign, "they still wanted to remove it." Patient D agrees to the surgery, expressing, "when a doctor becomes a bit suspicious, then I'm not sure what you hear." The physician's suspicion, not supported by laboratory evidence, translates into the patient agreeing to surgery.

I recall that my doctor had come. Now I—he was with somebody else, and I guess they had looked at the results. They said that they thought that it was benign, but they still want to, if I was okay with it, remove it. And that would remove any opportunities for the lump to change at any given time in the future...But I can honestly see where it still can be a very frustrating and overpowering experience. And it's frightening quite often to go to the hospital in the first place.

Patient $\mathrm{D}$ is fairly passive in this scenario; he trusts the imaging results, the biopsy, the professional interpretation and recommendation, although his language suggests that the diagnosis is somewhat vague. He readily agrees with the suggestion that the lump be removed. Yet, he calls the experience "overpowering," reflecting a sense of having no choice other than compliance. When awaiting results, still unsure if the lump is benign or cancerous, Patient $\mathrm{D}$ says, "I don't know how to react." This statement seems to assume that there is a correct or 
expected reaction. Patient D has been conditioned to be a "good patient," to be compliant, based on the (hopefully well-founded) belief that his physicians are acting in his best interests. ${ }^{106}$

Patient F struggles through his imaging experience, in part because appropriate information is not offered regarding what to expect during the angiogram, or specifically why it is being done. "I did not have any opportunity to ask questions at the angiogram, other than you're going to have an angiogram. 'What's that?' Well, we'll be checking the valves of your heart. I had all sorts of questions, but never had any opportunity to do so." Patient F recalls that each step of the procedure was explained as it was happening; however, he speaks of the experience as if he were a depersonalized object undergoing examination.

So they wheeled me in. "Move onto this bed. Now we're going to put this intravenous in. It's just sugar and water so you can just-we do it for everybody." And she said, "Now you're going to feel a little bit of pressure on your left thigh," which I then did. And she said—oh before that they anesthetized the surface. And she said, "Now we're putting a needle in, but you won't feel it because_-" and I didn't feel it. And she said, "Now we're going to move the tube, but you won't

\footnotetext{
${ }^{106}$ Most patients prefer to be perceived as "good," but circumstances can make this challenging. Patient A recalls a lung biopsy that kept her isolated at the hospital for over twelve hours, with her adult daughters anxiously waiting, because of an adverse reaction to medication given for the procedure.

So, when the doctor did come, he came to see me at nine o'clock at night to allow me to go down. He said that my pulse rate had been high. I could go now, but to be very careful because I had some holes in my lung. And I said to him that, "I was good, wasn't I?" And it was interesting. It was, again, it was that intuitive need. It wasn't snarky. It may sound snarky the way I'm saying it. But, "I was good, wasn't I?" A need to assert myself as an individual, this human being here needs to be heard. "I was good, wasn't I?" He said, "Yes. Yes you were.” Would you believe it? Patients need to hear that they have been "good," especially after a traumatic and exhausting day of unfamiliar events. Patient A intends to be "good," to be compliant, although her body has difficulty with the procedure. She interprets her question as, "A need to assert myself as an individual," and "to be heard"; however, other interpretations may also be valid. Her question sounds like a need for approval from an authority figure. It may also reflect the notion of illness as punishment; her body's reaction, and her medically induced separation from her family, may, on some level, be perceived as punishment for some unknown transgression. Assurance from the medical authority that she is "good," reduces or eliminates the notion that she is somehow being punished.
} 
feel anything. And at that point I said, "Do you think you can give me something to settle me down a bit because I'm really worried." [She complied with his request.] And she was taking it out, she indicated she was taking it out and she said, "Now I've removed it. We've put a bandage on your leg. We'll have to put pressure on it because we don't want it to bleed, and we'll move you out to the other room where we'll do that. So I was taken out to the other room. There was absolutely nobody in there and it was that big room again. But it was a different section that had been curtained off...Yeah, at that point they said, "We're going to put a brick on your leg to be the weight...It was quite obvious that the attendant was very anxious and annoyed that he had to stay there for the forty-five minutes because everybody was gone and all the staff were going out. And it was almost as if it was a public thoroughfare and they were all going out to go home, and this person had to stay there with me. He kept looking at his watch (grunt). But then the doctor came in, and he said, "Here are the results." [Interviewer: So you got the results the same day?] Yeah. As a matter of fact, if I look, I've got a pamphlet or a file in the cupboard there. I can show it to you. There's a diagram of a heart with all the valves and all the tubes. And he said, "This is ninety-eight percent blocked. This is ninety-percent blocked. This is eighty percent blocked. These two are a little bit blocked, but we're not worried about them." He said, "You'll have an operation." And he said, "I'll be contacting Dr. 
[...]", who's my cardiologist, "I'll be contacting Dr. [...] and he'll be making the arrangements. So he'll make the arrangements for the operation." Turned around and walked out... I would like to have asked him some questions about what I was holding. I wanted to ask him, "Well, when do we have to do the operation? How serious is it that I should-." I'd like to have asked, "Where will the operation be? Who will do the operation?" Well, he did say Dr. [...] will arrange it and so on. But I had all sorts of questions I would like to have asked him. But it was almost as if he was just part of the system that I was going through. And in fact I never did see him again. To this day, I can't tell you what his name is.

When asked how he feels about this imaging experience, Patient F responds, "Before, I was frightened to death. During, I must admit whatever they gave me did calm me to the point where I was able to... and I just lay there and let things happen." Without being fully apprised of what to expect from the angiogram, Patient $\mathrm{F}$ accepts the procedure, because his physician tells him it is necessary. Throughout the experience, he is clearly internalizing a great amount of fear, yet maintaining the actions of a "good patient." While his passivity gives the physicians and healthcare workers complete control in the situation, it also leaves them unable to support him psycho-emotionally. The only clue that he is in distress is when he requests, "something to settle me down a bit because I'm really worried." While he is given a sedative to reduce his anxiety, a more patient-centred intervention might have been to invite the patient to, "Tell me what you are worried about." Actively listening to the patient's question might have allowed the practitioner to put the patient's mind at ease, to develop a therapeutic alliance. The handling of this situation 
supports the status quo, in which the physician is in control, and the patient remains passive and, in this case, terrified. Commenting on real-time imaging, such as angiograms, Radstake suggests, “The success of real-time imaging depends on a delicate balance between patient's passivity and activity.... Actively attaching their bodies to the imaging apparatus, patients share in a collective, distributed agency" (90). She does not comment on the (un)willingness of the patient to share in this activity.

During his follow-up appointment, a few days later, Patient F has a much more informative and positive experience with his regular cardiologist.

...he had another copy of the sheet that I had. And he explained as this guy had, "You've got this blockage, this blockage, this blockage. We're going to clear it up. We're going to have the operation. Dr. Soand-so is going to do the operation...." Oh no, at that time, he said, "I'm not sure who's going to do the operation yet, because I want a specific doctor to do it, and I don't know if he's available for it, or when he's available." So I got a call the next day from his office, saying, "Yes, Dr. So-and-so is going to do the operation, and he will see you on this date, which was another week away. So I went to see him and the first thing that I thought about him was that he had a sailboat. So with that information, he was okay. [That this surgeon had a sailboat created common ground with Patient F, who also has a sailboat.] But it turned out he was the head of surgeons in cardiology. So, Dr. [...] did give me, arrange for me, the best people as far as my 
feeling about who was doing it. And this guy was fabulous...Once they resolved that I was going to have the operation.

Patient $\mathrm{F}$ feels confident in his medical team, so it is easy for him to agree to the surgery. $\mathrm{He}$ trusts his cardiologist, and this trust is extended to the cardiac surgeon his cardiologist selects. The cardiologist could also be seen to be exerting a professional power over his colleagues, allowing him to choose the most appropriate surgeon for this patient. Additionally, when asked if he has opportunity to have his questions answered during the follow-up appointment, Patient F responds:

Yup, yup, he wouldn't leave the office 'til I was finished...He was fabulous. He answered every question; wouldn't leave his office. He said, "Now is there anything else I can tell you about it? Is there anything else you want to know?"...And then he introduced me to the fact that next, whatever day it was, I would come down. I would meet the anesthetist. I would meet the nurse in charge. I would meet-well, I don't know, I forget now. But I would meet all the people. And we went down and they had half-hour segments. First half hour was an overview and giving out this book, and explaining what was in the book and what things you should make sure you read and so on and so forth. And then I had a half hour with the anesthetist, a half hour with the nurse, a half hour with some- - I forget. So I was ready for it and able to ask the questions I wanted to ask...And I remember the doctor said, the surgeon said, after we'd talked about the blockages and 
everything, he said, "Well, we'll take care of this stuff." You know, just that kind of attitude...Yeah. So it is possible.

Patient F's cardiologist takes time to ensure that the patient clearly understands the imaging results, and the recommended cardiac bypass surgery, using visual aids, as well as answering questions. The cardiac surgeon, as well as introducing Patient $\mathrm{F}$ to the surgical team, also assures the patient "we'll take care of this." Because Patient F feels fully prepared for surgery, he experiences little pre-operative anxiety. Conversely, due to the lack of communication regarding the angiogram, a fairly routine (if invasive) imaging procedure, his quintuple bypass proves far less frightening than his imaging experience. Reflecting further on his angiogram, Patient F says, I knew there was somebody standing there. I felt the presence. But I didn't know anything about him, 'til finally he said, “Oh, yeah there's the heart." And then he did the, "Uh-huh, yeah, okay, move it there, move it there. Okay." And then, "That's fine. That's all we need." And then, the next time I saw or had any involvement with him was when he came to the side of the bed to show me the diagram... So that, even though I'd had the angiogram, he had given me enough information to frighten me, but not enough explanation other than, "You'll have an operation."...I don't know whether he said, "Well, you'll have to have an operation," or "We'll arrange for an operation," or 'You'll be having someone...." But it wasn't in a "Let's volunteer to have the operation" kind of...

Clearly, Patient F feels compelled to agree with his doctors' suggestions, even when they are not fully or well explained. The problems Patient F experiences with his angiogram are the result of 
poor communication causing unnecessary anxiety, but they could also be perceived as a breach of medical ethics. Patient F would have signed an angiogram consent form. Having signed consent indicates that the procedure has been fully explained and understood by the patient, that the patient is providing informed consent, but this is clearly not the case here. Although patients do have the option to refuse to sign until or unless they fully understand to what they are consenting, most patients eagerly comply, even though they may not fully understand with what they are agreeing. Patients sign consent without understanding so they can procure the procedure or treatment that their doctors have indicated as necessary, but also so they can be perceived as "good patients."

Comparing his different experiences between the cardiac surgeon and the angiogram cardiologist, Patient F says:

Whereas the surgeon who did the operation, the first thing he said to me was, "Oh, we'll take care of this," which was assuring in itself...But the caring aspect was also with the surgeon who did the operation. Just because of his nature. He seemed to be caring, whereas with the angiogram, it was just an assembly line. ${ }^{107}$

\footnotetext{
${ }^{107}$ Even with Patient F's positive comments and the confidence evoked by his regular cardiologist and the cardiac surgeon, his ethical rights regarding the surgery still come into question.

There was no discussion. There was never any, "Do you want...?" Now when I went to see the surgeon, I had to sign forms of course. But up to that point there had never been any suggestion that I might not want to bother doing it... No, it was still a matter of, "You've come in because we're going to do the operation, and I'm going to tell you what I'm going to do." But it was also, "We'll sort this out. We'll fix this up." But it was never, "Would you like to...?" Never, never, never until I got the papers. But the reason you get the papers is, "Now, we'd like you to sign these so that the responsibility is yours." Well, oh yes, there was no doubt I was going to sign them, in their minds and then in their presentation.... When I went in to see the surgeon, I remember thinking, "Do I have a choice?" And I didn't discuss this with [wife and family].

In retrospect, Patient F, now fully recovered, is glad that he had the surgery, but it is clear that the patient's right to decide upon treatment is not always obviously respected. The patient's right to give informed consent sometimes seems to be glossed over, with physicians taking the lead in the decision-making process and not consistently
} 
Patient B offers an example of being talked into surgery by a physician, and then having things go wrong. She reports that she, "came to them [an orthopedic surgeon] with an MRI, and they took X-rays, to which I was never privy." The patient thinks that it might be time to "clean out my knee," as her physician has previously suggested.

And he said, "Oh, I think it's much more effective to do knee surgery, to do a replacement." And I said, "That's a little harder on the body." And he said, "Well, not really. People seem to recover very well from it." I never, ever heard what the failure rate was. I never heard that between ten and fifteen percent of patients never get over the pain. And I'm one of them. And I didn't know. And had I known, I would not have had the surgery because my golf was too important to me.

Upon reflection, Patient B does not feel that she was provided adequate information to make an informed decision regarding the surgery. The surgery diminished her active lifestyle of golf, tennis, and skiing, to one in which "I can't walk more than half a block without a cane." As a result of the surgery, Patient B finds that one leg is now an inch longer than the other, and that her knee joint no longer bends adequately. She confronts the surgeon, attempting to remedy the situation, but finds no satisfaction. Two years post-surgery, she approaches a more senior doctor at the same hospital who declares, "Everything about your leg looks fine." The physicians' perspective does not reflect the patient's experience, or her osteopath's findings upon examining

providing adequate information to ensure patient understanding. While patients generally have faith that their physician's decisions are in the patient's best interest, things do not always go as planned.

For instance, Patient A requires additional emergency surgery, just two days after a mastectomy during which she nearly dies due to a procedural oversight. Given the choice between the surgeon who performed the initial surgery, and a different surgeon, Patient A elects to stay with the first surgeon. This decision is clearly guided by a nurse who has established herself as someone to be trusted. While the other surgeon is probably fine, and many patients might refuse to have the first surgeon do the follow-up surgery, for Patient A, retaining the same surgeon is the right decision, and fortunately, the second surgery goes well. 
the post-surgical X-rays. ${ }^{108}$ Of her previously friendly relationship with the surgeon, she declares that the botched surgery, "spoiled the friendship, but it unnerved me terribly because I couldn't get answers, and worse than that, it was a breakdown of trust..." Patient B's mistrust of physicians is now apparent from her perceived need to research every aspect of her medical care online, to her decision to carry her detailed medical history to each appointment. ${ }^{109}$

Physicians' abruptness is sometimes viewed by patients as positive. Patient $\mathrm{C}$ is surprised by an MRI: "I said, I've never been told I'm having one. How come? And they said, well Dr. Soand-so said you are to have one." Again, the patient is not given adequate information to authorize consent; however, when the scan locates a cancerous tumour, she declares, "that was one time when I really blessed the machinery" because "that was discovered by mistake." She describes her respirologist as both "nice" and "tough as old boots." When she requests a delay in the follow-up appointment to play golf, he responds, "You're not playing golf." She suggests, “...he didn't over-explain. With this it was, 'You're not playing golf. You're coming back

\footnotetext{
${ }^{108}$ Patient B's frustration may be further fueled by her position as a financial contributor to this hospital; clearly, her contributions do not result in superior care.

${ }^{109}$ Patient A feels similarly shut down by an eye surgeon. Having experienced what she calls a "disconnect in my vision," she is referred to an eye specialist, who: ...examined me, and it was very swift. "Nothing wrong with your eyes." I said, "I don't have cataracts?" "No." "I've got this disconnect." Nothing wrong with your eyes."And I was out. So, you know that kind of person, you think, well the doctors have the information that they need. There's nothing physically wrong with my eyes. It has to do with something that is causing this in my eyes, but he's not going to discuss it. You get that feeling, well there's no point in expecting more from him, though another doctor could have been more interested and made the connection. "Sometimes these things happen," or, "It's not that..." He could have said it in another way. "There's nothing wrong with your eyes," two times. So you don't follow. There's no desire. Sometimes you just know enough is enough. You'll get what you need elsewhere.

Patients sometimes feel stonewalled by physicians. The specialist may have been overbooked that day, so irritated at having to see an additional patient. While the physician may feel frustrated, he or she is not living with the problem: the patient is. The patient is clearly concerned enough to request help in diagnosing and treating the problem, but as Patient A suggests, when a patient feels shut down, it may be best to take the problem elsewhere, to a physician who seems more interested in offering assistance.
} 
here.'... He let me know it was serious...but he didn't dwell on the fact." While Patient C clearly respects his authority, her respect is earned.

Patient A has a similar experience when a routine check-up turns up something suspicious; she is sent for a mammogram and rapidly to surgery. The doctor, "phoned the school where I taught during the day, and it was a Thursday. I had to close up my classroom by Friday, that meaning I handed it over to somebody else. Did it and was in the hospital on Monday. It was that swift. You don't get that kind of swiftness now." While clearly disruptive and perhaps alarming, Patient A is thankful for the speed and confidence with which her doctor handled the situation. Again, he made the seriousness of the situation clear, so the patient willingly complied. ${ }^{110}$

Patient E labels his healthcare workers the "treaters," saying, "I'm doing this [imaging] to enable the treaters to do what they have to do," even though the treatment "was making me sicker and sicker. It didn't make sense that this was helping because for me, I had very few symptoms of illness. So I was really quite comfortable, a happy guy, and the treatment was making me really, really sick, the chemo and the radiation." In spite of the physical discomfort, Patient E speaks well of his radiation oncologist and their tacit agreement when the heavy impact treatment was having on his body became clear:

The medical oncologist is a really kind man. Oncology is a hard specialization, because you're seeing such unhappy patients and you're often making their life horrible. It's already not looking good.

\footnotetext{
${ }^{110}$ Physician abruptness can also have adverse impacts, however. A doctor examining Patient $\mathrm{C}$ decades ago for university entrance, declared, "You won't live to be thirty." Now in her mid-eighties, Patient C boldly states: "I outlived this doctor, who was a she, doing the women students. And I've often thought, wondered, what damage she really did." More recently, Patient $\mathrm{C}$ had an incident with a physician who seemed distracted by someone outside her window; at that moment, she tripled Patient C's medication, putting her in hospital a week later. Patient C described this doctor as "totally inattentive and totally disinterested" - and human. Even doctors make mistakes; the problem is that patients usually bow to their authority, even when it is ill-advised to do so, or even when treatment causes worse symptoms than the disease state.
} 
But your treatment is just-you're not really going to get a big thank you note. You might, but .... So the relationship and the rapport is really important, and I felt with the chemo which was really - the chemo was clearly for me wrecking me. Wrecking me! And he knew that. And I was supposed to have had a third treatment, and I just couldn't endure it. And he knew that, so it was a trade off as to whether, you know, this could help, but at the same time how much can a body take... I didn't feel like I was failing, except I also wasn't going to be able to comply with the original plan.

Although Patient E states that he "didn't feel like I was failing," the following "except" undermines this assertion. The treatment plan was conceived by his physician for optimal treatment of the disease state; however, upon reviewing this plan during the course of treatment, and acknowledging Patient E's illness experience, the physician modifies the plan. Here, Patient E uses the word "failing" to acknowledge his inability to "comply with the original plan." To the patient, his inability to comply with the plan may feel like failure; however, to the physician, it is simply the modification of an intended course of treatment, indicating a significant power differential. Throughout his ordeal, however, Patient E trusts his physicians and his whole healthcare team. This trust is aided by their honest communication: “...my doctor did tell me that, you know, for six months he was taking control. And how often in life do you ever hear that? Never. I never hear that." Later in the interview, Patient E says, "I was told by my oncologist that, you know, he's going to be taking six months of my life away from me, at least. When he saw me most recently, he said, 'I should've said nine.'” In adult life, in contemporary society, in the west, we are rarely, if ever, placed in a situation in which someone else has complete 
authority over our bodies, especially for such a protracted period of time. This is the kind of power that physicians can wield, so patients hope that their doctors are using their power responsibly, and in the best interests of their patients.

Patient E acknowledges that patients often feel "unempowered" in the healthcare context. In addition to his comments about gender differences in support groups, Patient E also observes gender differences in the patient experience.

I think in terms of gender, it was probably, there is probably to some extent some differences between men and women's response to being a patient, to seeking help, to seeking support, to ask, to being vulnerable and to being empowered or unempowered. When somebody says, I'm taking your power away, and that was a helpful thing to say because all of the treatment is designed to make that patient want to balk and get the hell out because nothing is going to go right, then it's hard to feel any kind of power. And then when your body starts to literally lose its power, there's not much left in the tank to work with.

This observation underscores how powerless patients feel during prolonged aggressive treatment, regardless of their gender. The treatment seems more aggressive than the disease, and between them, they consume the patient's body and energy. Because of his throat cancer, Patient E found that assumptions were sometimes made along gender lines about how it might have developed:

Most of the cancers at throat and neck have been lifestyles. I'm always asked, “Smoke a lot? Drink a lot?” And the probability, although I haven't met them, is that a lot of the people, men, who 
have had this kind of tonsil cancer, have had lifestyle where heavy drinking, heavy smoking, not really talking, not really dealing with vulnerability, and not liking to trade off power or anything, not even wanting to go to a doctor. So when these guys show up at my radiation oncologist's door, with or without their spouses, they don't want to be there. They didn't want this in the first place. They don't want the treatment. They want it over with, and even in the most recent conversation I had with my radiation oncologist, he knows that there are so few, although he sees many people, men with this problem, there are very few who speak like I do. Most of them don't want to come. They don't even want to come back to that appointment, and probably a lot don't.

Men, according to this interview, seem more resistant to the patient role than do women. While their resistance may be read as a refusal to relinquish power and control of their bodies, this resistance to treatment can also have devastating effects on their health and outcomes. Patient E chooses to relinquish control and undergo treatment, commenting, "the treatment took so long and was so arduous." But having survived the experience, he asks rhetorically,

'Did I pass? 'You passed.' Am I still alive? 'You're still alive.' Am I palliative? 'I'll tell you when you're palliative.' So, I'm not palliative? 'I'll tell you when you're palliative.' So, I'm still alive, I'm breathing, there's lots of side-effects that aren't seeming to go away, but I'm still breathing and, 'You're not palliative.'” 
The patient needs this kind of reassurance after the treatment nearly kills him, but this passage also shows the power of a physician's words, as well as the demands on physicians of tending to the extremely ill.

Minor actions on the part of healthcare providers become comment worthy in Patient E's story. For instance, regarding the mask used to secure him to the table for radiation treatments, he comments, "They cut eyeholes for me which was a big help. And they cut a little mouth opening for me which again, was a big help." This seemingly insignificant action is noteworthy for Patient E and again, speaks to the incredible power of healthcare providers over patients. Without the mercy of these small slits cut into the mask, the patient would effectively be rendered blind and mute during treatment. Fortunately, Patient E finds that during his radiation treatments, "the staff...were pretty universally kind and empathetic, always ready to help, I must say. And never did I feel that I was being ignored in that way; if I needed assistance it was always there." While it is pleasant to feel that one is being treated well, in the medical context, good treatment has the added benefit of encouraging patient cooperation. Patient E continues, It's an institution where kindness is important, and I felt that there was a lot of that. It's remembering that it's probably pretty scary for the person, and these kindnesses can make someone a little less fearful. And if they're less fearful, they'll be more easy to work with when you have to get them on the table, which is where they have to go.

Patients are more likely to be cooperative if they feel they are being treated well, but even kind treatment can be a form of exercised power, a subtle form of manipulation. The complicated relationships inherent in medical practise are further complicated because physicians know, through their training and experience, that their treatment may make a patient feel worse in the 
short term, for the sake of better long-term results. The patient, who has no previous measure by which to judge his current experience, only knows that he feels worse than he did at the beginning of treatment, making it more challenging to continue trusting the physician and treatment process. Kind treatment can help soften the potential distrust caused by the physical ramifications of aggressive treatment. ${ }^{111}$

${ }^{111}$ While patients generally respond well when treated compassionately, occasionally healthcare workers resort to rough handling, sometimes leading to additional patient challenges. Patient A describes a post-surgical nurse who "force-fed" her. "She was very rough, and I remember being down, and her pulling me up into the sitting position, and now sick...I could see my daughter in the corner, and I could see her wince in response to my..." Perhaps the nurse wanted to ensure that the patient didn't develop complications from lying supine for too long. Perhaps she thought that the patient was malingering. Perhaps she had been asked to work an extra shift. Regardless, Patient A requires additional emergency surgery shortly after this rough handling.

Worrisome, is that both Patients A and C (almost a third of the patients interviewed) report that they nearly died from preventable surgical complications. Both were on anticoagulant medications, and neither was advised to stop taking their medication prior to surgery. During Patient A's mastectomy, she "almost bled to death during the operation"; afterwards, she repeatedly lost consciousness due to blood loss. She remembers, .... nurse brought me to with cool cloths or tepid cloths or something, which they probably do. But, you know I knew that this woman had saved me. She'd saved my life. I didn't know when I was out, but I knew when I was brought back that she was patting me. You know, to me that was wonderful. Now that, so that was maybe 4:30 or 5:30 in the morning when that happened and I went out. Then the nurses changed. I wasn't aware of it until this other nurse came in and I was going out again. And so I quickly said, remembering how I'd been brought back, because there wasn't time to say anything more, I said, "Get the other nurse." And she said, "You want the other nurse because I'm Black?" And I went out. Now that, again, there is so much you could learn from that. So much you can learn; the fact that in 1975 we're talking about, that nurse, the first thing she thought of, because it was part of her experience. "Is it because I'm Black?" Nothing could be further from my way of thinking. And the fact when needed, swiftly get the other nurse she would know... it was just sort of an instant thing. And that was a shocker.

Clearly, when a patient is in a vulnerable state, such as recovering from near-death surgery, nurses and other practitioners have even greater power. In this unfortunate case, the patient's attempt to exert some small power for the sake of her own wellbeing is misunderstood by the nurse. Fortunately, there are no negative repercussions for the patient from this incident, but as commented earlier, nurses are sometimes thought to treat patients poorly if the patient is perceived as "difficult," or in this case, racist.

Patient $\mathrm{C}$ is unlucky in having a surgeon, that on first meeting, ...he looked at me and hated me and I hated him, but if I'd known then what I know now, I'd have asked for anybody else - the janitor. And something went wrong in the operation. I've never known quite what, but I think he ignored the fact that I was on Warfarin and did nothing about stopping it. And so I think I began to bleed out, and he hurried to close up, and this was not quite properly done, and it leaked, and resulted in an abscess on the liver a year later which only identified itself by septicemia. I had a couple of nightmare years, and they're not clear enough in my mind. I do know they asked me if I wanted Last Rights when I came in with the septicemia. First they thought that I was an alcoholic. They were making a lot of assumptions. 
Different healthcare practitioners sometimes vie for power within the healthcare system.

For instance, imaging technicians have a limited scope of practice, which does not include

sharing diagnostic information with patients. Patient $\mathrm{C}$ suggests that the technicians she

encounters doing Doppler ultrasounds of her legs,

...are usually fairly uncommunicative, but I think that's their job

because they are technicians, and they get somebody to read it. And

that's fine. That's the way it should be. They shouldn't be talking

about...so they're not - they probably are experts by the time

Whether or not something went wrong during surgery, and whether or not the liver abscess a year later was the result, is irrelevant; Patient $\mathrm{C}$ believes her story to be true. This example underscores that sometimes doctors really do hold the power of life and death over patients. Clearly, this power must be wielded with great care, and yet as these examples show, appropriate care is not always taken. As Patient A indicates, in 1975, protocols about anticoagulants and surgery had yet to be established; however, shortly after her surgery, another patient sued a hospital because of a similar experience. Patient A reflects that she could have sued. Regarding doctors, she says, "There's such a concern that you're going to sue. I'd never do that. I mean, I was in a situation at the very beginning of all of this with the blood thinners, I could have; someone else did it. It wouldn't even have occurred to me to do that, even after that. I just had that sense that these things could happen." Although Patient A is philosophical about this incident, clearly not all patients were. After a few near misses, the medical profession established necessary protocols advising patients to stop taking anticoagulants prior to surgery.

More recently, Patient A avoids what might have been a problematic surgery. He [the surgeon] said, "Well, I can help you. I will do a sling operation." And he drew a little diagram. He didn't say anything, any side effects or the dangers involved. It wasn't that information. "I can fix you and we'll do it," this was in November, "we will in January." In April, it still hadn't been done, and I discovered that, in that interim, that there are court cases. I'm not saying against this hospital or this doctor, but pending because this particular operation is not as successful as one would believe. And then you get a lot of infections from it, which is the worst thing that could happen to me...A year. And then to find out-back to the original doctor, older doctor who I had had in the first place, that his questions immediately were, "Were you told? Is it going to be just the uterus? Is it going to be just the...?" You know, and the questions that he asked, no, I wasn't told any of that, and what was being used and all of that. And I thought, ah...

Clearly, inadequate information was provided to Patient A regarding her awaited surgery. As she is in her mideighties, allergic to many foods and medications (including most antibiotics), and this surgery has adverse affects, it might have had a devastating impact on her health. Yet her communication with the surgeon reveals none of the potential problems, and clearly does not take her specific health concerns into account. After nearly a year on various antibiotics to treat a chronic $e$-coli infection brought on by unresolved organ displacement, the surgeon declares that her situation is "not an emergency." Patient A concludes, "he didn't see it that way because he didn't really listen and understand that I had problems with medication. And always that's the first thing I say, because it is the truth. And I say it, and so often it hasn't been listened to...No one's paying attention." Patients want to feel well, and expect physicians to help them regain their health; consequently, they need to feel heard, and that their concerns are at least acknowledged by their physicians. Patients often want to feel more involved in their care than some physicians are comfortable with. 
they're - they really know what they're doing. But they don't have the piece of paper to allow them to tell you, and so it seems uncommunicative, but it's forced upon them. It's part of the system. You can't have technicians diagnosing and doing all the - telling you you're having triplets!

In Patient C's rationalization, while the technicians may be diagnostically capable, sharing information with patients falls outside their scope of practice, and so it would be inappropriate for them to do so.

Patient A's experience is somewhat different, in that she has heard results from technicians: "Oh, most of the time with ultrasounds, if the news is good the technician is telling you...So that's been very good." When questioned about the type of information shared by technicians, Patient A clarifies, "Their statements have been based on a previous ultrasound that I've had and, 'There's no change... from the last time.' It's been that kind of thing. It hasn't been any information that I felt should have come from the physician.” Technicians sometimes walk a fine line, risking giving away too much. When an ultrasound technician checks Patient A to clarify the site of an intestinal bleed, "right away, the technician, as soon as he knew what was what, he called out, 'Not cancer!' And so it's good when things are okay and they give you that immediate response. That's what I like about ultrasounds." When asked if she has received information from a technician that was contradicted by her physician, Patients A acknowledges that this has happened once after a goiter ultrasound.

And the technician gave the, "Oh," she said. "You must get in touch with your doctor. See your doctor." And she gave me information that sounded as if there was something seriously wrong. There was 
nothing wrong, and both the surgeon and my family doctor were very, they said, "This is not right. This should never have happened." I must say I worried about —it was the tone it was given to me in. It was very worrying.

In general, Patient A finds the technicians she has encountered very communicative and easy to work with. They are, "always talking to me. It's a very intimate space that you're in, and I felt that they were very much first rate in their communicating and directing to me...Certainly eyes were looking at me to get responses, and 'Are you all right?"' In her experience, technicians are aware of her comfort and the difficult demands imaging may impose.

Both advantages and disadvantages become apparent when medical professionals work in teams. Patient A recalls a power struggle between a surgeon and an interning medical student, in full view of both a nurse and herself as the patient requiring an imaging mediated left lung biopsy.

He [the intern] kept saying, "I can't find it. I can't find it.” He was in a panic because he couldn't find it. I couldn't say anything because he said, "Don't speak, don't move." And it would be, "Don't breathe," or "Breathe." You know it was that (laughs). He was very confident inbut he couldn't find it, and this went on for such — again the whole procedure should have been over in forty-five minutes. I would think that we were still going at it half-an-hour and he-I wanted to say, "Get the doctor!" The nurse didn't say anything; she just kind of looked at him and commiserated. But this was his test, so he was doing it. Well, the surgeon came in and he was furious. He said, "I 
can't hit the target. How do you expect me to hit my target?" because it's nowhere near where the nodule is. So he goes ahead and shoots the darts anyway, but he knows that he's going to miss. You know, I felt for him. But, would you believe it? He, when it was over, because he had spoken sharply to the doctor, to the young intern, the two of them, he put his arm around the doctor. Nothing was said to me. Put his arm around the young intern and they went off through the door together. The nurse said to me, "Roll over onto the gurney." That was my experience with the biopsy.

While the doctor empathizes with his young intern after the less experienced doctor fails in his task, no such gesture is made towards the patient. Although the intern is having difficulties, the patient is left feeling unsure about whether she has lung cancer, or her prognosis.

Sometimes the team member assigned to discuss diagnostic results with a patient is not ideally suited to the task. This was the case when Patient A received the results of an abdominal ultrasound in a busy surgeon's office. Because of other health issues, Patient A's scan was not "normal," nor did she expect that it would be. An intern given the task of telling her the scan's results was clearly unprepared.

...his reaction was "(gasp) did you know that you have a cyst? The lymphocytic cancer is right next to the pancreas?" All that I knew, so I really-I was absolutely nonplussed by it because I knew that my hematologist would discount that because he knew something other. So, no matter how diligent you might be, you get someone who is not the right person to be delivering that, the hot note. Very scary. And 
you have no way to really address that because you didn't see the surgeon after all. He left it to this fellow to do it.

This inexperienced intern is not yet familiar with the breadth of possible results, especially in older patients, nor with the powerful impact his reaction and words may have on a patient. Additionally, he neglects to ascertain the patient's existing knowledge about her health, likely findings, or potential co-morbidities, and he assumes that he is imparting new and startling information. Older patients have lived many years through which they may have sustained injuries, recovered from illnesses, or received wear and tear to their bodies that younger, "normal," healthy bodies are less likely to exhibit. As Blaxter notes:

Especially in an elderly person, it is likely that many forms of pathology could be found if a sophisticated search was made: old injuries here, a less than perfectly efficient organ there, narrowed arteries, worn joints, general loss of function with 'normal' ageing. The definition of 'abnormality', for this body, becomes problematic. Add to this the systemic nature of cancer, with possible spread throughout the body, and there is a strong possibility of suspicion arising over different bodily systems. (CVP 768)

A body can continue to function with a surprising number of "abnormalities." A patient can be a rich source of information regarding his or her own body and health. Accessing Patient A's existing knowledge of her medical condition would have allowed the intern to contextualize the findings for this individual patient, possibly making them less alarming, and providing a greater sense of patient competence in his professional abilities. 
In another imaging situation, examining a bladder blockage, Patient $\mathrm{A}$ feels ignored as the physicians discuss her case.

So he didn't give me information in the room where the machine was.

I went into another room to see him along with the doctor who had put

the tube in, and then popped in where the wonderful technician was

along with a student, and they looked at the computer. It wasn't turned

my way in this second room.

During the endoscopic procedure, the screen was facing her, and Patient A could have viewed her images in real time; however, after the completion of the procedure, the screen "wasn't turned my way." She was no longer privy to her images as at least three physicians gather around the screen to discuss her case. Physicians sometimes seem to forget that the patient is also present and listening. Not only can the patient feel ignored, but she may hear things that she only partially understands that can cause additional concern. ${ }^{112}$

\section{Medical Imaging Power}

...power reduces one to silence; truth does not belong to the order of power, but shares an original affinity with freedom..."

Foucault, The History of Sexuality 60

You can't read. You can't hold a book up. You can't do any of those things. And I don't imagine you could have earphones or anything

\footnotetext{
${ }^{112}$ Perhaps the ultimate expression of patients' awareness of their physicians' power is exhibited in "white coat syndrome." White coat syndrome, or white coat hypertension, is the physical response of approximately $21 \%$ of patients while in their doctors' offices, although their blood pressure in everyday circumstances is normal (Pickering et al, 225). Although Patient B has multiple health issues and spends much of her time with physicians, she says she suffers from "white coat syndrome. My blood pressure just goes up to 200 or 220." White coat syndrome is generally considered an anxiety response, or the body's way of acknowledge that the doctor has a great deal of power over the patient in the situation (Pickering et al, 225).
} 
like that...I don't know if that would interfere with the way the radio waves or what not... but something, a story or-because if it was a child, you could do a little bedtime story or something like that. Just something to keep your mind...

Patient G

Medical technologies, the machines that do the imaging and the images themselves, also wield a great deal of power in the medical communicative dynamic. Sometimes these machines seem to dominate the discourse between patients and practitioners; they seem to take on an authority of their own. While undoubtedly helpful, as seen in Chapter 2, their value is sometimes overrated or misinterpreted. Some interviewed patients commented on the power of technologies in the medical environment, both positively and negatively.

Medical scanners require the dedication of whole rooms; patients travel inside these huge machines for imaging. The size of these machines can be intimidating, and being unsure of how they work or what they do adds to this intimidation. Regarding her bone scan, Patient $G$ comments, "It's this massive great big huge machine. But it was explained to me beforehand by the technician. 'This is going to come very close to you, but it's not going to touch you.' So she kind of calmed me down a bit." Later, Patient G reflects, "But they were so close, so close. And I thought, 'What if that thing fell. It would completely crush me."' This may be an irrational fear, but it underscores the power attributed to machines; patients may be that frightened by their sheer magnitude.

While suffering from acute claustrophobia inside the MRI, Patient D reflects that it is "actually a bit of a relief having been through this machine," as the images produced indicate that the lump in his neck is benign. He suggests that his reaction is "just part of the experience, 
just the experience of being claustrophobic, and feeling not in control, and not wanting to let anybody else know that's the way I felt because... not supposed to feel that way." His statement begs the question that if the patient is not "in control," then who or what is? The physician is not usually present during routine imaging, having relinquished control to the technician; however, at the moment of imaging, the technician also relinquishes control to the machine. Both the patient and the technician necessarily subject themselves to the machine's needs, effectively giving it dominant control. Because of their desire to coproduce clear and useful diagnostic images, patient and technician cooperate, allowing the machine full reign to do its work.

Patient B comments on the somewhat defensive way that X-rays of her injured thumb were used by one of her doctors. She found this particular doctor "rude" in that he "didn't even say good morning." Without a word of greeting, "He switched the X-ray machine on, the X-ray screen on, and said, 'See? Your thumb isn't broken.”' This appointment was two weeks after the initial injury, and Patient B's thumb was causing increasing and constant pain, waking her at night, and making daily tasks difficult. Although six X-rays were taken, the doctor reviewed only one with the patient. Reviewing the whole X-ray series, Patient B's osteopath located a hairline fracture. In this case, the physician's interpretation gave the X-ray authority over the patient's story; "images can be used by doctors as 'proof' of the reason why they, rather than the patient's own perceptions, should be trusted" (The et al. in Blaxter CVP 763).

Patients who undergo multiple imaging appointments sometimes adjust to the technologies. After several CT scans, Patient G decides to dress for the occasion. "The girls laugh at me down at the hospital. They say, 'Oh you've got your CAT scan outfit on today.' Because what I do, I wear track pants, elasticized waist. No bra. I'm ready. I don't have to change. I don't even have to put a gown on. I just go the way I am. I'm all prepared.” While this 
makes her appointment quicker and more comfortable, it also acknowledges the machine's demands that she dress a certain way.

After a couple of uncomfortable imaging experiences, Patient A learns to request necessary assistance. Having held a difficult imaging position during a CT scan, the next time, she asks if someone can hold her arms in the correct position. Similarly, she is able to inform an ultrasound technician that she knows the procedure will make her feel dizzy; consequently, he checks in with her throughout the imaging process and helps her up afterwards. "I learned that if you've had an experience, you speak up, and you don't have to get into a state about it. It could be that someone is listening to you and they will respond." While she receives helpful responses to her requests for assistance, she only learns that she requires help after uncomfortable initial encounters with these technologies.

While sometimes intimidating or uncomfortable, imaging technologies can also provide comfort. After a body scan, Patient B asks for and receives a disc of the images. While she initially has difficulty interpreting the images, she comes to understand them and finds some useful meaning.

What you're seeing is where the blood is pooling in the body. They touched on, I saw a very brief report on the page, and it named various areas of the body where there was blood pooling, and I have three areas in my back. This is all the different disk areas in my back where the disks are in trouble. The feet, the knees, there was somewhere else in my body I can't remember off the top of my head. Nothing scary. It was actually comforting to find that there was an actual reason why I was having pain in my feet, is because there is blood pooling there, 
which is an indication of infection of some kind, possibly just

disintegration from arthritis. That's the most likely. I have, and I've

got the disc and I can go to it at any point and look at the rest of it.

And I've got a better idea now of how to read it.

Regardless of how correct her interpretation may be, Patient B finds comfort in having a disc of images, which seem to support her sense of trouble spots in her body.

Patient E's relationship to imaging technologies changes over the course of treatment.

Initially, he is focussed on starting treatment, rather than on the necessary tools moving him towards that treatment.

When I got the first CT scan, or MRI, I really wasn't interested. I

knew I was a person with cancer, I knew that these things were designed to get me moving toward treatment, and it felt like the scientists needed this information to formulate their treatment, and since I'm not really interested in the science, I don't know physics. I don't want to start learning now. I hope I have the best team possible and if this informs their decisions, it's good. So, I wasn't really interested in knowing. I already knew I had cancer; the CAT scan showed it. I was told what the results were, but in many ways it was like gibberish because it's not a language I even really understand, nor was it at the time a language I wanted to learn. But it enabled me to go forward. So, I had no idea what the CAT scan looked like. I had no idea what the MRI looked like. Further, I had no idea what my cancer looked like to those people who were looking at it. 
This attitude may be common among patients facing dire health concerns. Their focus is on treatment, on progressing towards health, rather than on the method of diagnosis or treatment chosen by their medical team. Over time, however, with additional exposure to the technology, and once the initial shock of diagnosis has passed, patients do sometimes become more curious; at very least, they may begin to learn passively through repeated exposure to the technologies.

In time, Patient E's response to the technology became friendlier: "I view the scans as part of my team." He continues, "Noisy members of the team, the MRI, but what they have to say is important data." By proposing that the scans and imaging machines are part of his healthcare team, Patient E endows them with power, as well as anthropomorphizing these inanimate objects. In terms of how this team member works with patients and physicians, Patient E says: "Technology helps both. I mean, it is a member of the team-it's just a big member." Patient E finds perceiving the technology as a big, noisy team member, helpful and necessary. During imaging, however, Patient E sometimes finds himself in a power struggle with the equipment's demands.

And then, for head and neck [MRI], my experience, you're often told don't breathe... and/or don't swallow, and it makes perfect sense to me. Don't swallow, oh I won't, because they're shooting images of this whole part of my throat [he gestures up and down over his throat], and I don't want an interruption... to be told this'll be, oh you know, four minutes, don't swallow. Then it's not so much scary for me, but it's like, okay, I want to be compliant because if I do what they [the technician and the machine] don't want me to do, then I'm going to get punished and I have to stay longer. So I want to get a good mark. I 
want to pass this test the first time, but the instructions are hard to comply with because they're so-well, for example, don't swallow more so than don't breathe. The “don't breathe," they don't say for four minutes, but sometimes if they need to get that-but the "don't swallows” are a long time. “Okay, swallow. Relax.” And I'm thinking, "No, this wouldn't be a place that I'd relax."...If I can, when I'm told, "Be still. Don't swallow," I can remember times when I have done some acting and there was one time where I had to be in the role of someone who was dead. And I could never tell what the director was seeing, and so I tried not to breathe. Or I tried to make my breathing as shallow and as minimal as I could. So, in that context I wasn't going to be told, you know, "We have to do this scene again because you're moving too much." But that turned out to be phenomenal practice for me when it came to the CAT scan, particularly the CAT scan, because it's quiet except for the sound of the machine. Because then I could get myself extremely still, and it worked for me extremely well during those thirty-five radiation treatments. Because I knew I was literally locked onto a table. I weren't goin' nowhere.... And being as still as I could be, as almost dead in a sense as I could be, would work for the technology, and it was a way for me to cope.

The technology, which Patient E understands as a useful technology, demands his physical compliance to do its work. When scrutinized by a reductive clinical gaze, the patient is 
objectified and rendered corpse-like. In an effort to make his body compliant for the technology, he plays dead. Similarly, Blaxter's Patient P comments that she: "Felt entirely responsible for remaining absolutely still, following positioning instructions exactly" (CVP 771). While these thoughts are noted and recorded by a subject, they clearly mark the actions (or inactions) of a subject endeavouring to be an object; the passive, docile patient, mimicking a corpse. Leder suggests that, "... the living patient is often treated in a cadaverous or machine-like fashion. We see this, for example, in the traditional physical examination. The patient is asked to assume a corpse-like pose, flat, passive, naked, mute" (Leder 22). During his MRI imaging sessions, Patient $\mathrm{E}$ takes this notion of becoming corpse-like to the extreme, knowing that it will best satisfy the technology, thereby allowing him the earliest possible release. In terms of radiation treatment, Patient E says, "you're lying on a table, and with this kind of cancer experience your head and neck and shoulders are literally locked onto the table. And then the machine goes, you know, does its work around you." During treatment, Patient E again acknowledges the passivity of his role, locked onto the table, while the machine works on him, taking control, and actually damaging his body, as it ultimately works for his benefit.

Patient E's recent MRI experience was not his easiest. The technician was eager to finish his shift and so perhaps rushed the procedure. What this means is that the technician put dampening headphones on the patient before the procedure, and then tried to communicate over the MRI's noise:

...put headphones on which makes it hard to hear, and then the equipment tends to be noisy. And, in my case, any instructions are spoken from another room. What he tended to do more often than not was speak over the sound so that I, and often because it was, "Okay, 
don't swallow," or "Swallow and relax," and I'm wanting to be a good patient, it's really important to hear what he's saying. What's the instruction? Is this the one where I'm supposed to not swallow, or can I now swallow and relax? His was the only time when the instructions were kind of voiced over the sound of the equipment, and a little soft, partly soft because I'm wearing headphones, partly soft now because my hearing has been adversely affected by the radiation. So that could've been changed. I didn't say, “Excuse me, could you speak first and then start the machine?" So I take some of the responsibility for it, but it was difficult because the instructions are precise, and if you can't hear them, and you want to do well... So, it would've been something that perhaps I could've told him and didn't.

The demands of the equipment make patient-technician communication difficult in this imaging context. The patient must wear headphones to protect his hearing, but this further complicates the communication around the noisy machinery of the MRI. In this case, perhaps unfairly, Patient E assumes partial responsibility for this communicative failure.

Patient $\mathrm{E}$ has a powerful experience when he finally sees his MRI images. In the midst of treatment, he was sent to a resident for an endoscope to chart his progress. As he sits in the waiting room, Patient E could see into a small office, and noticed images on the computer monitor.

...there was a head on the screen and I was sitting in the room and I was thinking, "I'll bet you that's my head, it's me. And he's [the resident] sort of warming up to come in and greet me. It turned out to 
be me. He came in and we chatted. He scoped me and was quite good about it — decent fellow. And at the end of it all, I said, "Excuse me, but is that my head on the computer that's just sitting in front of this office?" He said, "Yeah.” I said, "Could I see it?" He said, "Sure." And it was fabulous for me because it's, I don't know, I guess it would be like a looking for a fetus at an ultrasound. And I don't think I've ever actually been able to see one when I've looked at them, so you need to know a lot about anatomy and physiology to make a lot of sense of these maps. But what was really important for me is that he had the time one MRI, and the time two MRI on the screen-it's a split screen. And I could actually see where the cancer was at time one and where it wasn't at time two. And that was a wow for me, because it is a way that I learn [visually] ...So it was a kind of a blind spot for so long and so the good luck of that second appointment where I could see the image. So my sense of it is that, for me, having had the opportunity, whether I chose the opportunity or not, to actually see it, it being the CAT scan or MRI, might have been helpful because for me, simply if I had known. I knew that the cancer was in nodes that were down my neck, but that's Greek. Well, I liked the screen idea, because that's why I was doing all these things.

While Patient E's images are not offered for viewing, he asks to see them when the opportunity presents itself. As a visual learner, the chance to see the images has a powerful and enormous impact, enabling him to appreciate, for the first time, the work being done and the obvious 
benefits of treatment. A year after the conclusion of treatment, Patient $\mathrm{E}$ has additional images at the request of his radiation oncologist, to determine if the cancer is still in remission. "I'm happy to say, the first year, I passed. Translates that the cancer's not there." While other tests were probably ordered, imaging provides the ultimate assurance that the cancer has not returned.

\section{Patients' Power}

"Where there is power, there is resistance."

Foucault, The History of Sexuality 95

...the fact was that you have to express yourself in a way they are

going to see you're - that you're an individual, you're a human being, as they are.

\section{Patient A}

Finally, the power of patients is noted in the interviews, sometimes in surprising and subtle

ways $^{113}$. While patient power is sometimes expressed verbally, other expressions are non-verbal,

${ }^{113}$ While some patients find that being compliant smoothes their way, Patient A expects to be heard, to be listened to, and feels disappointed when she has to be more assertive with healthcare workers. When physicians speak to her in a reductive manner, she feels a disconnection and demands more.

The other is the listening. I have thought about this whole idea of, "Your numbers are good, that's fine." And I thought, "Ah, that isn't necessarily a closure to the conversation; it could be an opening." You know a doctor could be saying, "Well, your numbers are good" and not saying, "Is there anything you want to talk about?" But that expectation, if there was, that would be an appropriate time. So, it's good to, as I say, look at both sides, and not say this is the way this doctor is. If he's that way, it's easier to be that way, I think, if you have twenty patients still outside in the waiting room, if someone hasn't drawn your attention to it. But, I do feel that if the need was there, you express that need, I don't think that - the few doctors that I've had whom I felt were very, you were just in there for two minutes and then out, I still don't think that if I express something to them that they would stop and take notice.

If a patient expresses a need to know, to have more information, a physician has an ethical responsibility to provide further detail. Patient A intuitively finds a unique and powerful way to communicate her frustration to her physician. At age eight-six, she has multiple conditions, multiple physicians, and multiple medications. In addition to a form of leukemia, Patient A also has a prolapsed uterus, and has started having unexplained pain in her jaw and ears.

Appointments with a hematologist, an otolaryngologist (ear, nose, and throat or ENT specialist), and dentist yield no solution; the problem is not caused by her cancer, her ears, or her teeth. In her words, "I've got this kind of help, and 
I've got that kind of help. That's not enough." Extremely sensitive to medications, Patient A concludes that her pain is caused by an antibiotic she has been taking due to chronic infections from her prolapsed uterus.

And I thought, how unfair is this that I've been on antibiotics for eleven months, and now I'm going to bed angry at night? Angry. And so, my family doctor knew about this. I told her many times. But somehow, this day, I was saying, "You know it's just gone on too long and I was holding my hands on my jaws like this, cupped over my jaw, and I reached over and put my hands on her hands, on her jaw. And of course my hands would have been cool too; this wasn't an acting job, this was for real. I took that feeling, holding onto that pain and that intense feeling, and put my hand on her jaw and said, "This cannot go on. You are a good doctor," I said. "But I need to wear a pessary now. I am eighty-six. I am not having a sling. I want you to get in touch the original doctor. That was a Friday; Monday I had a call from [the hospital] to say that they would be seeing me on the Wednesday or Thursday or whatever it was. And I realized that it shouldn't have to come to that, and I shouldn't call it "coming to that." But because I was in absolute oneness with her and she with me as a result of that, but it came from me, and that was a good lesson, I think. But it wasn't something that you could have necessarily prepared in advance. That would have come out different. This was very instinctive and real, and she got the message. She heard and she understood. And by getting in touch with the doctor she let me know that she heard and understood. And that isn't something that you always have, the completing of that circuit of listening, that's it's been received.

Through a simple human gesture, Patient A connects with her physician in a new and deeper way. While very subtle, it allows her to take control in the situation, supporting her getting the appointments and care she feels she needs. It also provides a situation for the physician to confess, "I feel so fragmented; so fragmented" due to the demands of her practice. Some pysicians are reluctant to let down their guard with a patient to this degree.

During eye surgery, to correct a blocked tear duct and do a biopsy, Patient A asserts her personal power into a very controlled situation. Again, it is done very subtly, perhaps almost unconsciously. When she first meets the eye specialist,

...before I even opened my mouth he said, "Don't speak. I'll do the talking. Don't speak." And I hadn't spoken. Now, at eighty-six you know that there's something wrong here. He can't—-he's prejudging. Someone said the word: its ageist. But, absolutely that, you know? So then, the next time I had [my daughter] there, you know he was looking at her to do the talking, not at me at all, as if I absolutely did not count, but when that doctor, oh yes, this is important. So, there I am, [my daughter] and I, down in the hospital for the morning of the surgery. Well, when I walked into the operating room, the anesthetist had been out and talking to me and about what medication, because the surgeon himself had said, you know, he went through this whole thing immediately that I mentioned that I have a reaction. $\mathrm{He}$ says, "You know the difference between allergy and..." I say, "I said reaction." But immediately that, and giving me the whole spiel about, I know, but I listened dutifully. Now, I'm dutiful in that sense. I listen. I get their numbers; they've got mine, but I've got theirs. Anyway, as I'm going into the operation with the anesthetist, the nurses in there just lined up as I'm walking along. Suddenly, I realized that I am approaching, on one side there is this kind of semi-oval, and on the other side the same thing. They've lined up, two sides and they are smiling at me as I walk through into the operation. It was so beautiful. It was just wishing me well, and I guess they had. And the reason I realized that this is done deliberately is because I heard someone say, we must all do it together. And you know, it was a kind of reminder, because they maybe they'd done it before, but maybe they hadn't kept it up or whatever. But they were doing it. It didn't hit me until afterwards what they had done. And you know, it was like being wrapped around with love.

Incredible. But, I just thought, that is wonderful. That is a whole great more; an advance in medicine. Then, when I get under the local anesthetic for this, it's not very long; this man was a master at his job. There wasn't a word spoken, but it was 
through gestures or actions. Sometimes these are directed at physicians or healthcare workers ${ }^{114}$;

other times, they are more obviously a response to the technology.

palpable, the elegance of that operation. Again, this twit who was saying, "Don't speak, don't this, don't that...", and not even looking at me. But, boy, as a surgeon he was spectacular, and you could feel it. As I say, it was palpable. I spoke during the-I said, "That's so interesting. What are you doing?" I mean, I was very relaxed and laughing about it too. And, "Not now. Tell you after." So, unwittingly, he got my number too. He knew I was a human being. And immediately afterwards, I said, "Oh, the elegance was palpable, all of you. I could just feel it." So, he said to me the next time I visited, "It was so much fun" (laughs). This is the same man who said, "Don't speak. Don't speak." I just asked him the one question during the operation, but it was not deliberate, it was just — curious. It was interesting. You could feel, you try to imagine where they are now. Are you down at the end of my nose, or are you still up there? Trying to figure it all out...

In what seems to be a very tightly controlled surgical situation, Patient A intuitively brings herself some power, resulting in a positive relationship with this surgeon.

${ }^{114}$ The most direct verbal statement by an interviewed patient expressing her power was when Patient G, upset by the poor care patients were receiving from weekend nurses declares: "I've also got a mouth on me that I'm not afraid to complain." While she might have complained, as previously noted, she did not complain to the hospital's ombudsperson.

Patient D reflects on his philosophical approach to physicians:

Well, I sort of think of it this way, that, you know, if I'm going to get a new roof for my house, I'll ask for three or four quotes. If I'm going to have my car fixed, I probably will go to more than one spot. If I go to my doctor, and I get something that I don't understand or I'm uncomfortable with, I know enough now that I don't have to stop there, and actually my doctor would probably be on my side to say, "why don't you talk to somebody else? Here are a few other people that could help you out." And rather than just that blind trust that is still there sometimes. And knowing that you can go, and knowing that most doctors probably would encourage that in their own way for their own protection, people should do that and it makes you feel more at ease. It really does. I've got the best person to do what I need to do and I feel good about that person. Yeah. Or I have asked two or three and I, yeah, I got the same answer that I don't like, but they're consistent. It's reassuring that they do know their stuff, so...

Framing his physician encounters this way gives Patient D power to select, the way one might when getting a roof reshingled or a car repaired. Because of the scarcity of physicians in some Canadian jurisdictions, we may not feel that we have these kinds of choices, but Patient D feels that he does. Patient D also suggests, "I know enough that if the doctor that I'm seeing isn't great at asking, still there's information that I have to offer up that is helpful, I would just offer it up, whether he asked or not. And he can cut me off, or she can cut me off, if they feel they do not need that information." Clearly, Patient D is willing to negotiate power with his physicians.

As older patients, with increased practise communicating with physicians, some patients develop proficiency in exerting power in medical situations. Patient F, for instance, says, "I can ask questions." This assumes that practitioners grant patients opportunities to ask questions, which is not always the case. Patient C says, "If it's not explained, I ask questions. You know, I'm interested in what's going on that would be more likely in my case. They'd say, 'For God's sake, shut up!'” While she has a friend who incessantly complains about doctors' attitudes, Patient $\mathrm{C}$ counters this, saying, "I, on the other hand, don't give a damn. It's not my business to do this. And I go merrily in, and my experience has been very, very good." Sometimes patients get whatever they expect; leaving the medical side of the equation to trained personnel, works well for Patient $\mathrm{C}$. When asked why she has not asked more questions in uncomfortable situations, Patient A responds, "I've asked myself why I don't ask those questions more frequently, but I think, and I think perhaps it has something to do, again, with my responsibilities to myself, and working things out for myself. The best learning I have is that which I find out for myself and it's just reflecting on situations." Although Patient A feels comfortable asking questions, she sometimes hesitates, perhaps due to an 
When Patient E asks his radiation oncologist how he prefers to be addressed, the physician allows the patient to control this aspect of their relationship. Interestingly, Patient E opts to address him as doctor, yet still finds this mode of address empowering. ...the radiation oncologist, I remember asking him initially whether he wanted me to call him—would he be comfortable with me calling him by his first name, or doctor, and he left it up to me. But I could see that in many ways he wanted to be called doctor, that he was a doctor and a scientist. A radiation, like a physicist, and a physician, were extremely important roles for him and the way he could be helping me. He's just a lovely guy. But I think for him, it's really important

intellectual pride of finding her own answers. When pushed a little further on this matter, she responds, "I was certainly expressing how I felt right along. But it wasn't, it was like, well what else can I do?...Well, and I think too because of the way people feel about medicine and hospitals, and their fear of being sick or dying, you know all of that plays into it, doesn't it?" So even when a patient feels confident to ask questions, and needs answers to feel more certain of her situation, she may not ask, for fear of the answer.

Patient $\mathrm{C}$ has on occasion voted with her feet; she has opted not to return to physicians she felt were rude or inadequate to the task: "Because the first one, he was rude to me. You know, my reaction was to almost get up and walk out, except that I have a little bit of common sense. But, the second one, I just made a mental note that is not one I would go back to." While Patient C's response seems reasonable, sometimes circumstances of time and place may not allow this option. Simply walking away, without giving verbal or written feedback to the physician, also means that the physician loses the patient without knowing why. The physician has no opportunity to do better next time, because he or she may be unaware that his or her behaviour is problematic for patients. During a difficult specialist's appointment, the physician discovered that he did not have all of Patient C's medical information:

And then at one point he accused me of, yeah, he asked me, "When did you have your knee surgery?" And I'm feeling in my bag for these papers, and I don't see very well, and I had this little problem too [indicating her splinted thumb]. So I'm fiddling, which I hate fiddling; it makes you look a hundred years old. And he said, "Well maybe it's your mind you're losing." I mean, that was inexcusable, and I looked at him. I almost stood up and I said, "How dare you speak to me that way. There is nothing wrong with my mind." And I handed him the piece of paper and I said, "Perhaps if you'd be kind enough to read this. The information is there." He read it, and he said, "Why didn't I have this?" I said, "I don't know why you didn't have it, because the recommending physician had it." I was absolutely livid. I was shaking, I was so angry. And then he said, "I don't think you have a neuropathy."

And I said, "I don't think I do either, but what do you think I have?" And he said, "I think you have arthritis." I said, "Isn't that interesting. Nobody has yet asked me what I thought, and arthritis is what I think."

In this situation, a stressed physician seems to be taking out his frustration on the patient regarding an administrative oversight. The patient's assertion of her power initially encounters resistance, but ultimately leads to agreement. The patient, in this case, is well prepared with detailed documentation, but still finds the situation upsetting and very poorly handled. 
that the science, that the physics, helped him to get to the room to help a person like me, and the medicine helps him to stay in the room with a person like me. So the joining of those two roles were important for him. However I want to call him, that's okay. But what I found with the radiation oncologist is that he always knew that I was the patient, and I always knew that I was the patient, but it was so obvious that it just, for me it was, I guess, empowering in a way.... I think if I had to just sort of give a broad brush stroke, and I just did, he always made me feel that I was important to him.

In this complex moment, Patient E requests power, and is then offered power by the physician. While the patient feels empowered by the offer, he chooses to address the oncologist formally, in part because it seems important to the physician, in part out of respect, and in part because it helps clarify their roles. ${ }^{115}$

As previously noted, Patient E is a visual learner, and his "breakthrough" moment was when he finally had opportunity to view his MRI scans. Prior to this, he tried to illicit visual information from his oncologist.

Earlier on I had said to my medical oncologist, "You know, visual is kind of a way for me. Could you draw them?" Because I had had all these treatments and I know what they're doing, but my head is

\footnotetext{
${ }^{115}$ When Patient E is told that his prognosis is good, and he will likely be cured, he says, "I was compliant [with the radiation and chemotherapy], not wanting to fight," because, "from the beginning, I had a sense that I wasn't likely to be palliated." While healthcare professionals have the power to pronounce his condition curable, Patient E has the power to decide what attitude to bring to treatment. "I was prepared to do what I needed to do, and I had a sense that if I did it well, or as well as I could do, then I might actually find myself cured of this." So in this case, the patient consciously chooses to be compliant, hoping that it will lead to a positive health outcome. At the same time, Patient E feels: "I think the person with the illness, or the person needing this stuff also has some responsibility to inform the person [healthcare worker], because it's possible they will remember." Patients need to verbalize their requests if they expect their needs to be fulfilled; healthcare practitioners may not be mind readers.
} 
anchored to a table. I had no idea really what they're doing. He said,

“Well, I can’t draw” (chuckles). Well okay, I asked.

In this case, the patient tried to exert a subtle power to get information that he felt he needed. Unfortunately, this attempt was shut down by the physician's self-perception that he "can't draw." While the physician felt unable to draw a diagram for the patient, something that is often used to good effect, he could have offered explanation by showing the patient his MRI or CT images.

Patient E comments that, "with certain staff I felt very human and invisible, very present and invisible. With some staff I felt mostly invisible." Patient E's response is similar to Blaxter's, which discusses, "the 'virtual patient', of the possible disappearance of the person behind the images" (CVP 764). This sense of invisibility could also be read as a sense of powerlessness; however, Patient $\mathrm{E}$ is very aware of his caregivers and makes astute observations about their place in his care. “...as this treatment goes on, I was getting just more and more wasted, so it was really apparent to whoever was seeing me from day to day that this is tough. And it's tough for them too, because they're watching someone deteriorate. They're not really seeing a good news story." His observations may be a way of taking back some personal power, when, "I feel so unempowered through so much of this... empowerment isn't something you're feeling. You know, your body's kind of wasting away.” During his imaging appointments, when he was disempowered by both personnel and machinery, Patient $\mathrm{E}$ offered a strong gesture that consciously took back power in his "unempowered" situation:

One of the things that I asked for was, not a blanket, a sheet; something to cover me because it's quite chilly in the room. And often I was asked if I would like something, but I always asked if I could 
have something. It made me feel I had a little bit of power in that moment, and it was a bit more comfortable because the rooms tend to be chilly and you're wearing this silly costume, you know, a gown. Maybe your socks and your underpants and that's about it. And again, if I tried my best, and my best was good enough, they seemed satisfied. I didn't get called back. Sometimes they had to do a repeat of a section, but again, not a sense of judgement, it's just a relief. Yeah, I'm back in, I have some power now I'm back in a costume I wore when I came in.

The simple act of requesting a sheet to cover himself and keep warm during imaging, provides a small sense of power to Patient E. Once imaging is complete, being allowed to dress in his street clothes reconstitutes his personal sense of power before re-entering the world outside the hospital.

\section{Conclusion}

This chapter has explored Foucauldian notions of power in the medical imaging context. While it may seem obvious that physicians hold power in this situation, surprisingly, power sometimes shifts to the technologies themselves, and may even be usurped by patients in some subtle or unexpected ways. Even when patients seem passive and compliant, they are constantly watching and listening, aware of the context dynamics around them. They may be responsive to power struggles between personnel, and know when they, as subjects, are being ignored as objects or cases to be solved. Patients generally want to understand diagnostic findings, which may include imaging data; furthermore, patient understanding is required for informed consent prior to 
treatment. Because patients generally trust their physicians and medical team, they willingly subject themselves to imaging or other procedures, but their compliance is enhanced when they fully appreciate why something is recommended, and know that their physician's desired outcome matches their own. Small acts of kindness towards patients can have huge positive impacts. Male and female patients may behave differently in the medical environment; however, more study is necessary to make this claim.

The machinery of imaging tends to exert control over personnel and patients; both parties are subservient to the machine's demands to co-create the desired images. Although patients may or may not acknowledge their fears of the imaging technology or medical environment, sometimes causing their compliance, they may still feel overpowered or overwhelmed by its enormous presence. While images have the power to rule a diagnosis in or out, they can also be used by physicians to wield power over patients. If the image fails to provide supporting evidence of the patient's bodily experience, physicians often feel that the patient is mistaken, rather than the image. As discussed in Chapter 2, imaging results and their interpretation are fallible. Imaging findings, even when dire, may provide a sense of relief at finding an answer. Negative imaging results may, correctly or incorrectly, provide relief that everything is fine; or they may cause frustration when they fail to support the patient's lived experience. Physicians must listen to the patient's experience, even when imaging results are negative. Patients who are routinely imaged to track the course of treatment become more familiar and comfortable with the machinery.

Perhaps surprisingly, patients can have power in what may seem a disempowering situation. Patient E provides the strongest conscious example of patient power in the imaging 
context; however, most other interviewed patients reveal moments in which they exert power, sometimes quite subtly, within the medical context.

This is the last of three chapters reviewing and analyzing information from seven interviews with older patients, interrogating these texts with four primary questions regarding their emotions, physical sensations, thoughts, and notions of power before, during, and after their imaging experiences. Additional information regarding their communication with medical personnel has also been included in extensive footnotes. Based on these interviews of older individuals' imaging and communicative experiences, simulated patient roles were developed with supporting medical images for physician encounters. These encounters are analyzed in the following chapter. 


\section{Chapter 7:}

\section{The Impact of Imaging Technologies}

\section{on Physician-Simulated Patient Communication}

Care is not, you know, let me hold your hand, you know, snuggle you up. For certain people like myself, what we do is, is more, well, it $i s$, it's rational, it's focused, and it's not about falling apart and blubbing. It just isn't like that. ... Hospitals go through a lot of criticism because, "Oh, they were uncaring, they didn't care about me, blah, blah, blah." That's bollocks, actually. That's absolute crap, okay?

Eddie Chaloner, Consultant Vascular Surgeon (Lewisham Healthcare NHS Trust, 40-45 minutes) As argued in Chapter 1, Galen and Descartes were both foundational to the biomedical model. They both championed reason over emotion, considering the latter to be an unbalanced aberration (Dear 69; Wooton 40). As clearly indicated above, some physicians still hold this to be true. What the biomedical model risks disregarding is that caring does not equal "blubbing"; in fact, patients might feel alarmed to observe a strong emotional response from their doctors. What Dr. Chaloner and his ilk fail to recognize is first, it is understandable that a patient might have a strong emotional response to a life-altering health situation; second, regardless of patients' emotional responses, they should still feel heard and their questions must still be answered. As indicated in Chapter 3, physicians must communicate successfully with their patients to create trust and compliance, to promote healing, to reduce fear, and to reduce the prospect of ethical breaches. 
Chapters 4, 5, and 6 focus exclusively on patients' perspectives of medical imaging encounters with physicians and other healthcare workers. This chapter analyzes encounters between physicians and simulated patients playing roles partly based on experiences described by the interview participants, supported by images and their documented findings as detailed in Chapter 3 and Appendix D. Initially, some discussion of the challenges of recruiting physicians is provided, and then a brief discussion of some unexpected findings from the recruitment process. This is followed by an analysis of the physician-simulated patient (SP) encounters with imaging present using the Observation Protocol developed for this study (see Appendix E). The final section analyzes the brief post-encounter interviews with physicians and simulated patients using semi-structured interview questions (see Appendix F).

\section{Physician Recruitment}

Please be advised that I will be out of the office until Monday, January 7 2013, and will not have regular access to my emails. Any urgent issues regarding my Cardiology patients should be addressed with the Cardiologist on-call at the Toronto Western Hospital. Happy New Year!

Automated e-mail response from recruitment request The recruitment of older interview participants, as well as simulated patients, ${ }^{116}$ was fairly simple; however, physician recruitment proved extremely difficult. As a consequence, this stage of research was nearly abandoned. It is worthwhile to document this process, as it revealed some unexpected findings. The initial plan was to recruit between six and nine physicians, with a mix

\footnotetext{
${ }^{116}$ My work since 2000 with the Standardized Patient Program at the University of Toronto's Faculty of Medicine simplified recruitment. As I have worked with numerous individuals in this program, I was able to choose experienced SPs in an appropriate demographic range for my research.
} 
of specialists and generalists, allowing two or three encounters per case. Due to recruitment difficulties, these expectations were scaled down; ultimately, four physicians were recruited, and each saw two cases, providing eight encounters.

Beginning November 9, 2012, the first seven physicians invited to participate were those who had previously expressed interest in the research. Although four returned communication, a much higher ratio than later recruitment drives, ultimately, none was able to participate in this research. One had relocated outside Toronto; one stated that he was no longer interested; one was too busy—a sentiment heard several more times during recruitment. While the fourth initially looked promising, study location proved to be an impediment. Relocating the research to her hospital office would have required hospital ethics approval, in addition to the university ethics approval which had already been granted. This individual was also dismissive of the $\$ 25$ honorarium plus parking offered to assist with this research, stating " $\$ 25$ is not a real incentive for physicians." The researcher had, perhaps naively, hoped that physicians would see the intrinsic value of the research, and would therefore be willing to participate. While the dollar value of this honorarium might seem meagre to those in medical research, humanities research budgets are usually much tighter. This study was completely funded by the graduate student researcher, with funds allocated from an Ontario Graduate Scholarship — money primarily used for tuition fees and living expenses. Of the physicians who did ultimately participate, two of the four refused to accept the honorarium or a parking refund. Ultimately, none of the physicians who had initially expressed interest participated in the study.

The second round of recruitment entailed creating a database of potential participants, considering the relevance of their specialities to the roles that had been created, their proximity to the research facility, and the public availability of their professional e-mail addresses. 
Between November 11 and December 3, 2012, 174 individual e-mail invitations were sent, with negligible response. With the holiday season approaching, the timing did not seem optimal for recruitment, so this activity was halted until January 2013. Recruitment began again on January 4 until January 15, 2013. In addition to some new leads, previously contacted recipients who had not responded were sent a second request; 151 individual invitations were e-mailed. In total, 325 individual e-mails were sent, the vast majority of which received no acknowledgement. While positive cold-call response rates are usually about $10 \%$, e-mail response rates are considerably lower. Quoting the 2012 report from the Direct Marketing Association, American marketing firm Loop Demand indicates that positive responses from researched e-mails generally run about $.12 \%$. The recruitment effort for this study was not successful, as statistically it should have returned stronger results. While twenty-four negative responses were received, and a dozen email or telephone conversations ensued, ultimately, this extensive and time-consuming effort garnered only a single physician participant. One cardiologist was recruited at this time, who participated in the research in February 2013.

The other three physicians were recruited through contacts at the Standardized Patient Program (SPP) in the Faculty of Medicine at the University of Toronto. Twenty-seven invitations were sent in late November 2012, garnering no results. A further 378 invitations were sent by the SPP office in January 2013. This recruitment drive did not consider the physicians' specialities, or their proximity to the research facility. Instead, these are physicians who work as examiners during medical licensing examinations. While they are all in the GTA, they had much further to travel to the research facility than those on the researched recruitment list. The primary difference is that these physicians have a proven dedication to medical education and research. This dedication arguably skews the research results somewhat; these physicians are likely more 
aware of patient-centred interviewing techniques, as they are part of an educational system teaching these skills.

Recruitment was further complicated by the availability of Ryerson University's Daphne Cockwell School of Nursing Lab, where the encounters took place. The researcher is grateful for the support of the Nursing Lab in making this facility available at no charge to someone outside the department. Similar facilities in Toronto would have cost in excess of $\$ 1000$ for the duration of the study, and would have been considerably less flexible. Additionally, removing the research from Ryerson's campus and into a hospital, where similar medical education facilities exist, would have required hospital ethics approval. Gaining this approval might have added another year to the research process; the research was intentionally designed to avoid this delay. The Nursing Lab is, however, for the use of Ryerson's Nursing students, so research encounters had to be scheduled outside normal Lab hours when the space was available. While the Nursing Lab was very generous in allowing the use of their facility, and ethics approval was limited to campus, these limitations also made it more challenging to book physicians who have limited availability.

\section{Recruitment Findings}

regrets-no time. good luck!

A physician's response to recruitment request

The recruitment process itself revealed findings, some not surprising, but others unexpected. Owing to the time of year, numerous automated out-of-office replies were received; some of these individuals did respond later. As indicated above, the availability of lab space was 
sometimes problematic for scheduling such a busy population. A selection of physician responses includes:

$>$ "Sorry, not available any of those days."

$>$ "Sounds interesting but will be away until 20th of December."

$>$ "Though I am interested, all those dates are not good for me."

$>$ "I am gone that whole week : (

In all cases, these physicians were offered alternative dates; generally there was no response to follow-up e-mails.

The hectic schedules of physicians were apparent in many responses, for instance:

“Thank you for your invitation. My schedule is very busy until next year so I won't be able to participate."

$>$ "I'm sorry—I'm just too swamped. Good luck"

$>$ “Thanks for the email, it sounds like an interesting project. Unfortunately I don't have time to participate."

"Between clinical, administrative, and teaching responsibilities, I have absolutely no free time. Sorry"

> My apologies but I must decline your invitation to participate in this research project. My schedule is absolutely full for the time being. I wish you the best of luck with your research.”

D "This sounds like a highly interesting initiative, but I'm overburdened right now and so simply don;t have the time to participate. Wishing you the best in your project.” (sic)

"Due to many current commitments, I am unable to participate. Sorry I could not be of help at this time." 
"good luck with this but I don't have the time to participate at this point"

"No sorry, cannot do this as I am out of the office 3 days that week and have clinics two other half days. That only leaves me one day to do the whole week's work."

While some messages might be considered a brush off, the rushed nature of several responses, the details offered in others, as well as their tone, indicate that many of these individuals may be highly stressed and overburdened.

Three physicians who were unable to participate did, with the researcher's permission, forward the recruitment e-mail to their colleagues; another physician made the invitation available to his residents. Although the researcher anticipated a stronger response when the invitation was forwarded by a colleague or superior, this did not prove to be the case. No recruits were garnered in this manner.

The most surprising finding was that many physicians do not read images in their practice; they rely exclusively on radiologists' reports. ${ }^{117}$ As previously noted in Chapter 2 by Birkelo et al., the recommendation is that images "be read independently by at least two interpreters" (365), a recommendation given numerous times over the decades. The obvious opportunity for this second look is when a physician receives the images and report from a radiologist. While not as comprehensively trained at image interpretation as radiologists, physicians are trained and examined on their abilities to read and interpret images. Yet it becomes apparent from some responses that this review does not happen. Some physicians were vague, stating:

\footnotetext{
${ }^{117}$ This is, at least, the case in contemporary Canadian practise. Medical Humanities conferences in Europe have provided the researcher with opportunities to converse with medical doctors and radiologists from other jurisdictions, who were shocked that images were not reviewed by family or general physicians during their conversations with patients. This informal finding bears additional research.
} 
“Thanks for inviting me but I wouldn't review images in usual practice" (Family Practitioner)

"I'm sorry — my practice does not incorporate imaging technology for diagnostic assessment with patient care.” (Family Practitioner)

"I am sorry I am not the right person to interview as I do not routinely use imaging in making patient decisions." (Cardiologist)

While imaging may not always be an appropriate diagnostic tool, it seems surprising that physicians, especially a Cardiologist, would not sometimes refer to images. The following two responses make their approach more explicit:

"I would not say that my practice routinely includes reviews of imaging beyond the written report...” (Family Practitioner)

“I don't think I can help. I don’t review images myself only reports." (Family Practitioner)

Another prospective recruit, a Family Practitioner (FP), was contacted by phone. During this conversation, she declared that she, "only read reports. I don't look at the images."118 As previously suggested by Reiser in Chapter 2, this delegation of responsibility to another practitioner with no knowledge of, or relationship with, the patient, could be seen as an ethical breach. At very least, this delegation of responsibility could be interpreted as a dereliction of patient care. The FP could provide a second pair of eyes for image interpretation, as recommended; however, this is not what happens in practice, at least not currently in the GTA.

The final unexpected finding was in the form of a longer response from a full-time Emergency Department (ED) doctor. While he declined to participate in the study, he did offer the following: "You may want to try this kind of work in the ED setting sometime. 15\% of our

\footnotetext{
${ }^{118}$ My own Family Physician also supported this, saying, "I never even see the images—just reports."
} 
patients are over 65, and we bombard them with all kinds of information all the time. To make matters worse, we are not their regular MD, so there is no continuity of care!" It would be fascinating to be a "fly on the wall" in such a context; however, the prospect is also daunting, both ethically and personally. What this note makes clear is that even while working in this context, some doctors are aware of certain shortcomings in the system in which they practice.

\section{Analysis of Encounters}

If you get to ninety and you have a heart attack or stroke, well unfortunately, that's sort of life catching up with you.

PH B during encounter with SP C The encounters between physicians and SPs with imaging present took place on February 20, March 20, and March 22, 2013. All encounters took place at Ryerson University's Daphne Cockwell School of Nursing Lab at 415 Yonge Street, Toronto, Canada. In each case, the consent form, which had previously been forwarded via email, was discussed and signed. The physician was then handed a file folder with the chart note and images of the SP he (all recruited physician participants happened to be male) was about to encounter, and told to take as much time to review the file as seemed appropriate (these chart notes and images are included at the end of each simulated patient role in Appendix D). When ready, the physician then entered the examination room in which the SP was waiting. A laptop computer, with the same images as printed in the folder, was also available in this area. Physicians were told to use the images as they would in practice, and, as much as possible, to do and say what they would normally do and say during their encounter with the SP. The audio and video of all encounters were recorded on CD-ROMs for later review. The researcher was also able to monitor each encounter in real time through one-way glass and take notes. 
The following analysis uses the Observation Protocol developed for this study (see Appendix E) with notes taken both during the encounters, and upon repeated viewings of the recordings of each encounter. Each of the four roles was encountered by a General Practitioner (GP) and a specialist, and the manner in which each of these physicians encountered the same roles were comparatively analyzed. The two cancer roles, with character names Lee Berkowitz (benign) and Dana Jones (findings), will be analyzed first, followed by the two cardiac roles, Skyler Hughes (benign) and Chris Anderson (findings). Table 1 below indicates which physician encountered each role, and which SP portrayed the role, as well as their demographics.

\section{Table 1: SP-Physician Encounter Schedule}

\begin{tabular}{|l|l|l|}
\hline \multicolumn{1}{|c|}{ Role Encountered } & \multicolumn{1}{|c|}{ SP Encountered } & \multicolumn{1}{c|}{ Physician } \\
\hline \multirow{2}{*}{$\begin{array}{l}\text { Lee Berkowitz } \\
\text { (oncology, benign) }\end{array}$} & SP B (female, mid-50s) & PH A (Oncologist) \\
\cline { 2 - 3 } & SP A (male, early 60s) & PH C (GP) \\
\hline \multirow{2}{*}{$\begin{array}{l}\text { Danc Jones } \\
\text { (oncology, with findings) }\end{array}$} & SP A (male, early 60s) & PH A (Oncologist) \\
\cline { 2 - 3 } (cardiology, benign) & SP B (female, mid-50s) & PH D (GP) \\
\hline $\begin{array}{l}\text { Chris Anderson } \\
\text { (cardiology with findings) }\end{array}$ & SP B (female, mid-50s) & PH B (Cardiologist) \\
\cline { 2 - 3 } & SP D (female, mid-60s) & PH D (GP) \\
\cline { 2 - 3 } & SP C (male, mid-60s) & PH B (Cardiologist) \\
\hline
\end{tabular}

\section{$\underline{\text { Lee Berkowitz }}$}

Lee Berkowitz presents as a sixty-six year old healthy hypochondriac. During a routine examination, his/her doctor finds a "shadow" on the lung X-ray, and subsequently sends Lee for a CT scan. The imaging experience creates additional anxiety, and Lee is certain that the results will be devastating. (See Appendix D for complete role, chart notes, and images.) This role was encountered by PH A (Oncologist) and PH C (GP). While both interviews were fairly patientcentred, these two physicians use somewhat different approaches. PH A tends to use more close- 
ended questions, while PH C has a more conversational exchange, using a mix of open and closed questions. PH A asks questions such as, "You do not smoke, right?" (an assumptive question), and "Do you have any coughing, or shortness of breath, chest pain or anything like that?" Although PH C asks similar questions, additionally, he pursues relevant questions around the patient's social history, such as, "Anything in your family history?...Anybody in your family smoke, or in your house smoke?...Are you exposed to second-hand smoke...What type of work do you do?...So you were never exposed to any outside things. You never worked on a farm or anything like that?" He even asks, "Have you travelled anywhere recently?" Even though the responses to these questions are negative, they cast a much broader net for the differential diagnosis than those asked by PH A.

PH A's encounter becomes more conversational once he has gathered the initial information, and is offering his diagnosis and next steps. Although Lee is anxious by design, all these roles include explicit instructions that they be physician-driven, rather than SP-driven. SP B perhaps overplays the anxiety affect, asking numerous questions beyond the role's parameters. Throughout the interview, PH A remains very patient, answering Lee's questions with a smile; however, the SP pushes this interview to continue long after it has ended organically. One concern that emerges both near the beginning and end of this interview is that the SP is not aware of any earlier chest X-rays (a detail not included in the role). PH A mentions twice that he would like to locate any earlier X-rays, as they might provide useful comparative data.

Both PH A and PH C direct their gaze primarily to the SP; the only exceptions are during the few minutes when they were focused on the images or chart notes. During the explanations of imaging findings, both physicians use medical language, but immediately define the terminology in lay terms, without being prompted by the SPs. For instance, PH A points to 
something on the monitor, saying "This is just what we call the hilum, a collection of blood vessels." Turning to the hard copy images, he continues, pointing to something and saying, "It is not what we call speculated, like having fingers sticking out." Here he also gestures with his fingers splayed to illustrate his meaning. Similarly, PH C immediately defines lipoma as "a deposit of fat." When he mentions pulmonary hematoma, he immediately follows it with, "it is not a cancer," then continues to define it as, "a collection of, almost like, connective tissue." Giving this definition further detail, he says, "they [radiologists] look at it and look at the borders, they look at the calcification and things like that. They felt that it was more in line with a hematoma rather than something carcinogenic." PH C offers a more detailed explanation of the findings, outlining why the radiologist might have reached this conclusion.

Throughout these detailed explanations, PH C routinely checks in with the patient to ensure understanding, by simply asking, "Okay?" This is enough to allow the patient to pursue a question if s/he has one. Conversely, PH A does not verbally check in or ensure patient understanding until the end of the interview. He does, however, seem very observant of the patient's body language; whenever she looks as if she might ask a question, he invites her by saying, "Yes?” SP B pushes her agenda throughout the interview, so perhaps the PH feels reduced need to invite questions. Both physicians consistently answer their patients' questions, and in terms the patients seem to understand. Similarly, both physicians respond to the patients' anxieties, taking time to address their concerns. Lee's challenging opening line, "It's cancer, isn't it?," is acknowledged and deferred by both physicians. PH A says, "We will discuss it, and we will talk about it in a few minutes," indicating that he prefers to ask a few questions first to familiarize himself with the patient. He then says, "We'll get to that, right, I promise you, in a few minutes." He tacitly acknowledges the patient's anxiety. PH C does not immediately 
acknowledge the opening question, prompting SP A to repeat the opening line. PH C then says, "Well, that's what we're here to discuss." After a moment's conversation, PH C overtly acknowledges, "I know that you're anxious to hear the results." This is followed by the broad opening question, "How've you been feeling?"

With the overly anxious SP B, PH A is quite emphatic and repetitive in his assurances, stating when viewing the images with the patient, "Those are what we call calcium, and when we see calcium in the mass, the chance that is what we call benign — that is non-cancer-is going to be higher." When the SP double checks that he means that the mass is not cancerous, PH A emphasizes, “No. Non-cancer. Not cancer.” As SP B keeps repeating the possibility that it might be cancer, PH A later responds, "The doctor who was reading the CT scan, and myself, we feel that the suspicious index is low that we are dealing with cancer. This lump is not a cancer." Later still, he again states, "We are not dealing with a cancer." Towards the end of the interview, relating his diagnosis back to the images, $\mathrm{PH}$ A states, "So, in the X-ray there, there are some good features suggesting that it is not a cancer." Throughout these multiple reinforcements of the good news, PH A remains patient.

The primary difference between the PH A-SP B role-play, and the PH C-SP A role-play, is that in the latter encounter, once the possibility of cancer is discussed and dismissed, cancer is never again mentioned. PH C states, "When we see a mass in the lung, okay, it could be a number of things, okay? Now, definitely cancer can be one of the differential diagnoses, okay? But it could also be something quite benign." He then definitively states: "It is not a cancer." While PH C takes SP A's concerns seriously, once they are dealt with, SP A feels no need to revisit the concern with additional questions. 
Non-verbal communication is also a subtle, but note-worthy, factor in both interviews. PH A uses the images on the monitor to explain the results to the patient, causing him to shift towards the monitor, away from the patient. This positional shift means that he is no longer facing the patient, but is sideways to the SP. After the explanation, although he turns away from the monitor, he does not resume his previous posture, but remains sitting somewhat sideways to the patient. While the images are being explained, SP B leans slightly towards the monitor, at one moment pointing to something on the screen. By contrast, $\mathrm{PH} \mathrm{C}$ opts to use the hard copy images from the patient file, rather than the monitor. He removes the images from the folder, and shifts closer to SP A; in response, SP A moves his chair closer to PH C for better viewing. Both lean their heads towards the images during the explanation, which only lasted a few seconds.

The primary focus of eye contact is similar in both interviews, remaining on the patient. The only time the patient is not the physicians' primary focus is when they are viewing and discussing the images, or distracted for a moment by trying to get the imaging technology to move to another view. Similarly, in both encounters, the patients' primary focus is on their physicians, except when viewing the images. PH A tends to use hand gestures, but he does not use as many in this encounter as in his encounter with Dana Jones portrayed by a male SP (see below). PH C also uses broad hand gestures. No increased anxiety or agitation, beyond that required for the role, is noticeable in either SP A or B during these encounters.

PH A's encounter with SP B lasts 24 minutes, and ends when the researcher knocks on the door, once it becomes apparent that the SP feels obliged to protract the interview indefinitely. The encounter between PH C and SP A lasts only 7:30 minutes, and ends naturally. During each encounter, the time focussed on the images seems minimal. After 2:15 minutes of the interview, PH A and SP B shift to the images, remaining there for 2:22 minutes; however, even during this 
time focussed on the images, PH A still glances at SP B, for a total of 45 seconds. Also during these few minutes on the images, for about 18 seconds, $\mathrm{PH} \mathrm{A}$ is focussed on the imaging technology, trying to move from the X-rays to the CT scan images. These seconds are largely silent, with him saying only "so, um" several times. Once the images are explained, PH A only glances occasionally at the images, for a total of 45 seconds during the remainder of the encounter. Relying on the hard copies, PH C spends much less time on the images: 23 seconds. He flips through them fairly quickly, pointing to different views of the same concerning feature, then returns to explaining the images' meaning and next steps. As the SP makes no move to stop him or refer to the images again, presumably, this is adequate time to understand their meaning.

Other than the images, the only other point of focus is the chart note in the file folder. $\mathrm{PH}$ A spends 38 seconds of the 2:25 interview opening looking at the file, but usually engages with the patient. PH C spends a similarly brief time looking at the file near the beginning of the interview: 34 seconds, with a further 9 seconds after the images are explained. As previously noted, in both encounters, eye contact is primarily with the patient. Similarly, patients primarily focus on their physicians, unless directed to examine the images. SP B spends 1:38 minutes on the images when they are being explained. She glances at the physician twice during this part of the interview, but does not receive eye contact in return. Similarly, SP A's focus moves to the images when directed by the physician. During the few seconds that PH C spends viewing the file folder, SP A continues looking at him, but receives no eye contact in return. These few seconds when the SP looks at the $\mathrm{PH}$ and receives no eye contact in return do not to agitate the SPs in any observable way, and seem inconsequential. 


\section{Dana Jones}

Dana Jones presents as a sixty-seven year old taciturn fatalist who deeply distrusts doctors. S/he went to a walk-in clinic two weeks previously after prolonged productive coughing and shortness of breath. X-rays revealed a suspicious mass in one lung, so s/he was sent for a more definitive PET-CT scan. The scanning experience was fine; however, the radioactive tracer injection prior to the scan was poorly handled, further enhancing his/her distrust of medical professionals. Dana has smoked a pack to a pack-and-a-half of cigarettes per day for fifty years. S/he has a strong family history of lung cancer, but no intention of quitting smoking. Dana has also experienced increased fatigue and weight loss in the last month, although these details have not yet been shared with any physician. (See Appendix D for complete role, chart notes, and images.) This role was encountered by PH A (Oncologist) and PH D (GP). Unlike the Lee Berkowitz role discussed above, in the Dana Jones interviews, a sharp contrast is observable between the two physicians encountering this patient.

Similarly to his previous performance, PH A offers a fairly patient-centred interview. Although he asks many closed questions at the beginning, his opening question requests SP A's permission: "Can I ask you a couple of questions first?" Other questions include: "I was told that you started coughing up some blood about two weeks ago?...Have you lost any weight?... And how are you feeling, say, for the last...when were you last well?...And do you get shortness of breath?...How far can you walk before you become breathless?... Is the shortness of breath getting worse, or just staying about the same?... Otherwise, you don't have any problem swallowing?...Do you have any chest pain?..." These are all questions to help clarify the doctor's understanding of the patient's medical condition. He then proceeds to ask questions regarding the patient's social supports: "So, are you married?... So, who is at home with you?" If Dana 
requires serious medical intervention, these supports will be necessary. Shifting his body towards the monitor, PH A asks permission a second time: "So, if it's okay with you, I want to go through all what is the test results." Because Dana is rather reticent by design, this interview is necessarily very physician-driven; the discourse is dominated by the physician, and his mostly closed-ended questions. Throughout the interview, however, PH A responds to SP A's questions, explaining terminology and adjusting his language to ensure patient understanding. Additionally, PH A remains very positive and hopeful for a good outcome, in spite of the findings. After a detailed discussion of the results and treatment options, he states: "I think that based on all the information so far, I am quite optimistic. You have a localized cancer. Most likely it is lung. It is an unfortunate thing, but the good news is, it seems that it is confined within your lung. That is potentially curable.”

Conversely, the encounter between SP B as Dana Jones and PH D was highly problematic. Although PH D had the same written and verbal instructions as the other three physicians, and understood that the focus of the encounter was communication around imaging technology, his interview reflected a bullying attitude towards the patient around issues of agreeing to have a biopsy and quitting smoking. As a result, the interview became quite acrimonious. Additionally, technical problems during this interview made it sometimes difficult for the researcher to hear or transcribe dialogue, although enough conversation, in addition to the body language, is clear to make the confrontational tone obvious.

PH D begins with very open questions: "What do you know?... So, do you have any idea of what's going on at all? Any concerns at this point?" Although superficially these seem like very patient-centred questions, to the researcher, after over seven minutes they begin to seem like delaying tactics to avoid discussing the findings. When SP B, eager to leave the doctor's office, 
says, "I want you to fix me up if it's possible and just get on with it," PH D responds, "It's a lot more complicated than that." While this may be true, PH D makes no attempt at empathy. He has already determined that the patient smokes, and that her primary risk for lung cancer is selfinflicted - knowledge that seems to create a very negative attitude towards the patient. Unlike his colleague, PH D consistently uses very grave language, saying things like: “I'm concerned, very concerned about this. It looks like a tumour." He then states, "I do not make a diagnosis without a biopsy. I need a biopsy" and refuses to make a definitive diagnosis; PH D uses the word "biopsy" at least ten times during this encounter. The combined effects of having raised the patient's fears, and refusing to offer a diagnosis, completely shut down the interview about halfway through, leaving physician and patient sitting in awkward silence for over a minute. When they resume, and PH D again refuses to give a diagnosis without a biopsy, SP B begins grasping at straws, asking if it might not be cancer. PH D agrees that it might not be cancer, creating false hope for the patient.

Only after their stormy silence, towards the end of the interview, does PH D begin asking social questions, such as “Are you married? Do you have any kids?" As this line of questioning continues, however, it becomes apparent that he is not asking these questions to ensure patient support, but rather to find new leverage to try to manipulate the patient into agreeing to a biopsy: "What would you say if your daughter said she had a mass in her breast?" In the course of trying to persuade the patient to agree to the biopsy, PH D states, "We're not talking about something that's optional," also saying, "It's not going to be comfortable." Not surprisingly, his persistent use of negative language does little to convince the patient to agree to the biopsy. The effect of PH D's insistence that SP B have the biopsy is apparent; the patient is clearly annoyed by midinterview. Her agitation is clear from her tone of voice, and PH D only adds fuel to the fire, 
saying in exasperation, “That's what I'm going to help you with!” PH D never acknowledges the patient's fears or attempts to negotiate a suitable outcome. At one point, he states, "Clearly I'm not going to send you to someone I don't think is any good," to which SP B responds, "I hope not." PH D counters with, "Why would I?" his hands open in exasperation. This is the highest point of antagonism in the room. After a brief time-out, PH D continues, "Can I guarantee that things are going to get better? No. On the other hand, what's going to happen if we don't do something?" While the interview reflects PH D's belief that his approach is the only path to potential success, he fails to get the SP onside using bullying tactics; he does not understand that there is no "we" in the room. Rather than creating a therapeutic alliance, his continuous strongarm tactics fail to deliver desirable results.

PH D also pursues smoking cessation during this encounter, even though the patient is a life-long smoker with no interest in quitting. Again, the physician's approach is to try to bully the patient, and the exchange becomes argumentative. When the patient insists that she is not somebody who could just quit, PH D responds, "I disagree with you" and continues his monologue. Finally, the patient simply states, “I don’t believe you.” Clearly, no therapeutic alliance is created during this encounter; any hope at establishing trust is dashed by PH D's approach. When the patient says, "It'd be hell for me [quitting smoking], but I'm sure it wouldn't make that much difference," PH D counters: “I don't want you smoking post-surgery, because it's going to be very difficult. You're going to be going through a lot of pain and suffering." He becomes quite agitated, his frustration apparent through heavy sighing, slapping his hand on his knee, before continuing, "Ultimately, it's your choice. I have to be realistic in terms of saying to you here's what you can expect. When you're in the hospital, you won't be allowed to smoke. Again, it's your life. No one's going to take that away from you. It's up to you, but for the short- 
term, my strong advice is to quit smoking." He then offers her prescription medication to ease the symptoms of smoking withdrawal.

What is most confusing about this encounter is that while refusing to give SP B a definitive cancer diagnosis, PH D simultaneously places her in post-surgical "pain and suffering" in hospital. As discussed later, he also mentions "staging," a term specific to cancer diagnosis. As an observer, the message seems very confusing. PH D ends this encounter saying, "It's been lovely meeting you." It is, however, clear from his tone and facial expression that he is quite annoyed with the encounter, and so the sentiment of his words seems insincere.

Both PHs A and D generally retain eye contact with their patients throughout the interviews. Once PH A clarifies his understanding of the case, and announces that he is going to go through the test results, SP A states, “It was kind of an unfortunate experience... I don't like hospitals very much. It was a bit of a weird experience getting that scan thing." Rather than moving towards the findings, PH A acknowledges, "Sometimes it's a little bit frightening, right?" This acknowledgment of the patient's fears is a simple, yet effective, way of offering empathy. PH A then requests the patient's permission a second time before going through the test results and motioning towards the monitor. His focus is primarily on the monitor and the file as he facilitates the explanation of the findings; however, his attention returns to the patient when he begins to discuss the results' meaning. Conversely, PH D largely ignores the imaging results, spending only about one minute looking at them with the patient.

Similarly to his Lee Berkowitz interview, PH A uses medical terminology, but immediately explains its meaning in lay terms, or checks to verify the patient's understanding, or answers the patient's question with a simple explanation. For instance, when PH A states, “All we can see is what we call increased uptake..." SP A interjects the question, "What was that you 
just said?" PH A repeats, "Increased uptake" and then explains, "That is the black dot, right? So the black dot is where the radioisotope - they give you the injection during the scan. They concentrate in that area, and when there is a concentration, so there is, it becomes darker." $\mathrm{He}$ then looks at the patient to ensure that this explanation had been understood. Seeing that the patient has no further questions, he continues, indicating points of concern on the image. When PH A switches to a different view, a cross-section of the chest, he first ensures that the patient understands what he is seeing from this new perspective. PH A then indicates, "We see something abnormal in the PET scan, and there is something there. And this is what we call a lymph node, because you can see is just a very small spot." SP A asks, "What did you call it? A what?" At this point, PH A leans towards the patient and explains, “A lymph node, a gland...do you know what is a lymph node?" When the patient responds, "No, not really." PH A explains, "Okay, so, the lymph node is a gland in your body. Say, when you have a cough or the flu, sometimes you feel it in your neck." In this way, he is able to relate lymph nodes to the patient's experience. SP A responds, “Oh, so when mom said you've got swollen glands, that's what she meant?" The doctor responds, "Exactly," before continuing his explanation of why having a growth in a lymph node might be quite serious as it might spread a problem throughout the body. PH A indicates that "we can see a lump" and that "is suspicious"... "correspond to a growth." PH A is careful when using the word cancer. "Nowadays the PET scan is a useful test for us to detect lung cancer." When SP A reacts physically, leaning back into his chair, PH A responds by gently saying, "Well, cancer, and especially is lung cancer, because you do have a smoking history, and there is also a family history, so you are at risk."

While PH A is careful to explain medical terminology and ensure patient understanding, PH D does not make the same effort. He uses a mix of lay and medical terminology, but the 
medical terminology is not defined, unless the patient requests further information. For instance, when PH D uses the medical term "lymph nodes," similarly to PH A no explanation is offered until the SP requests a definition. When prompted by the patient, SP D offers, "We have them all over our body and they react to infection." Unlike PH A's explanation, this definition is not something that the patient can readily relate to, yet PH D fails to ensure the patient's understanding. Similarly, at 14:15 of the interview, PH D says, "You have to be staged." This is medical language specific to cancer diagnosis; any patient unfamiliar with the medical language of cancer may be unfamiliar with this term. Again, PH D offers no further explanation until SP B asks, "What does that mean?" While PH D answers the patient's questions, sometimes in ways that the patient seems to understand, he seems to find it frustrating when the patient requests these definitions. As with her previous role-play, SP B proves more talkative than the reticent Dana is intended to be. With PH D, this is perhaps a good thing, as SP B continues to ask questions until the answers are clear, making it obvious that the doctor is not communicating well with this patient. PH D clearly has his own agenda, and is unconcerned about negotiating the patient's agenda. He certainly does not offer SP B the careful explanation and definition of imaging results that is offered by PH A. Not surprisingly, PH D did not check in with the patient or invite questions during the interview; he only invited additional questions at the very end of the encounter.

By contrast, as shown above, $\mathrm{PH}$ A has a more conversational encounter, routinely checking to ensure patient understanding. PH A also adjusts his language to the patient's comfort level: at one point, he begins to say pulmonary, then instead says, "breathing test"; later, he says "lung doctor," rather than respirologist. PH A invites patient questions twice at the end of the interview, as well as saying, "I'm sorry I give you a lot of information today." Additionally, he 
offers a contact number for the office, scheduling for a return appointment, and also suggests that the patient write down any further questions, making them easier to remember during the followup visit. While both SPs ask questions during their encounters, PH A is clearly more conscious of ensuring patient understanding.

In both encounters, the physician's primary eye contact is with the SP. Again, PH A uses hand gestures to advance the conversation. For about 5:20 of the encounter, PH A is somewhat turned in his chair, explaining the findings using the images on the monitor. SP A also leans forward towards the monitor during part of this explanation, but leans back in his chair after about a minute. Although he does not lean forward again, his focus remains on the images. During the explanation of the images, PH A's back is to SP A; however, once he has explained the images, he turns fully to the patient to deliver the diagnosis, and maintains strong eye contact with the patient throughout this explanation. PH D also uses some hand gestures to explain the diagnosis. Similarly, he moves his chair closer to the monitor when viewing the images. Although he does not move the chair back, he does sit back in the chair once the explanation is completed. Near the beginning of the interview, PH D tosses the file aside, and does not review it any further. He delivers the difficult diagnosis within the first few minutes of the encounter, leaning towards the patient to do so. PH D leans back into the chair about halfway through the interview, but for less than a minute, before leaning back towards the patient. This attempt at creating a more intimate encounter receives no physical response from the patient, who maintains her position with her back against the chair.

While SP A's encounter with PH A seems generally comfortable, this SP often has his arms crossed; however, this posture is appropriate to Dana. SP A understandably becomes less comfortable, his body stiffening, with the suggestion that surgery to remove part of his lung 
might be required. Conversely, SP B encountering PH D becomes progressively more tense and agitated as the interview progresses. She too starts with her arms crossed, as is appropriate for the role, but her body becomes progressively tighter throughout the encounter, and she never leans towards either the images or PH D. In both cases, the patients' focus is primarily on the physician, unless they are viewing images on the monitor. SP A's focus remains on the images as PH A explains their meaning; this is the case even when PH A tries to make eye contact. Similarly, SP B focuses on the images longer than PH D. The Dana Jones role includes an optional prompt: "Can I see the pictures? It might help me understand." By the 12-minute mark, when the images have still not been reviewed, SP B draws them to the physician's attention saying, "Is that me?" and motioning to the screen. If not for this patient prompt, the images might have been omitted entirely from this interview.

PH A's encounter with SP A lasts 17:47 minutes and ends naturally. PH D's encounter with SP B is 20:27 minutes, and ends when the researcher intervenes by knocking on the exam room door. The decision to end the interview was made for several reasons: a) SP B prompted the interview to continue several times past its natural conclusion, which was out of character for Dana; b) a sense that the interview was not going to accomplish anything more, and that both parties were frustrated; and c) continuing technical problems affecting the audio in the control booth. The time spent focussed on the images, or on the file, was quite different between these two encounters. PH A spends 27 seconds in the file during the opening 2:40 of the interview; PH D spends only 7 seconds in the file before tossing it aside at the beginning of the encounter, but reviews it again after the encounter has ended. Between 3:16 and 8:39 of the interview, PH A spends a total of 3 minutes focussing on the images. Although he is turned towards the monitor, he regularly glances back at the SP, either in response to a question, or to see how the patient is 
doing. Once the images have been explained, PH A only glances at the images for a total of 35 seconds during the remaining minutes of the encounter. From 11:48 until the end of the encounter, his attention is on moving forward with treatment options. During the explanation of the images, the SP's focus sometimes remains on the images for a few seconds longer than PH A's; the physician is looking at the patient, while the patient is still focussed on the images. While PH A focuses on the images for 3 minutes of the encounter, SP A focuses on the images for 3:35. As with his first interview, PH A spends about 10 seconds of the interview trying to make the technology work to give him the desired image. PH A's use of images is very different from PH D's. PH D spends only about one minute on the images, broken up with glances at the patient. While the SP is also viewing the images during this minute, her eyes remain on the images during this time, even when the physician is looking at her. Similarly to SP A, SP D continues viewing the images for a few seconds longer than the physician.

\section{$\underline{\text { Skyler Hughes }}$}

Skyler Hughes presents as a compliant and affable sixty-nine year old, who is in generally good health. In the last six months, s/he has experienced occasional mild chest pain and shortness of breath during physical activities. Skyler mentioned this during a regular check-up and his/her doctor said it was "atypical chest pain" and sent him/her for a coronary CT angiogram (CTA). Although the imaging experience was very claustrophobic, it was otherwise uneventful. (See Appendix D for complete role, chart notes, and images.) This role was encountered by PH B (Cardiologist) and PH D (GP).

PH B and SP B have a pleasant conversation, a very patient-centred encounter, and even share a laugh. PH B begins by asking open-ended questions to better understand the patient's 
experience, for instance: “And you've been getting some chest discomfort or breathing troubles?" He then facilitates the patient's narrative by acknowledging her story with the intermittent use of "okay." His questions continue with, "And, when did it tend to come on? Was this when you're resting, when you're walking?... And what did it feel like if you had to describe it?... Did it go anywhere, down the arm, through the back, to the jaw?...And how many episodes of this have you had?... Anything you could do to make it better?...And, you've otherwise been in good health?" With each open-ended question, he allows the patient to share her experience, sometimes offering possible descriptive words (e.g., is the pain, "Burning, sharp, pressure, stabbing, tight, heavy...”), retaining eye contact, and facilitating her story. In a similar manner, PH B gathers the family medical history.

SP B opts to use the role's prompt to direct the conversation towards the images: "Can you show me the pictures? They might help me understand better." While PH B agrees to do this, before doing so, he explains what they will be looking at and how it is diagnostically useful. Understandably, this section of the interview is dominated by $\mathrm{PH} \mathrm{B}$ explaining the imaging findings, with SP B sometimes acknowledging and saying "okay." SP B offers several questions out of curiosity, which PH B answers in lay terms, taking this opportunity to educate the patient on the heart's structure and function. According to the findings associated with these images, Skyler has "lipomatous hypertrophy of the interatrial septum" (Mullins, Case 12100)_essentially a benign fatty deposit on the wall separating the heart's right and left chambers. PH B explains this finding with care, ensuring patient understanding, and also emphasizing that it is nothing to worry about, saying, "It's a normal thing... Now, this is nothing to be concerned about...It's just something that we accidentally picked up on the scan." He repeats several more 
times that, "this is nothing to be concerned about" through the remainder of the encounter. When the patient keeps asking questions to ensure understanding of this benign finding, PH B explains: Let's say we talk to a thousand people. Say, five or ten would have this. But you could have it your whole life and it wouldn't do anything, it wouldn't mean anything. It's not going to have any impact on you. It wouldn't change anything at all. It's just something we've picked up on the scan. So, it was more or less just to let you know, “Oh, okay we've seen this particular thing." I don't want you to be worried about it. Nothing at all to be concerned about. Okay?

After assuring the patient that the imaging finding is of no consequence, however, he also indicates that it is unlikely that the lipomatous hypertrophy is causing the patient's mild chest pain and shortness of breath. This is the patient history and findings accompanying these images; however, the researcher must acknowledge her own shortcomings and complete reliance on information from medical education websites. It is probable that other images, as well as other diagnostic tests, were administered to the original patient, but not shared online, to reach this diagnosis.

PH B indicates that he is not satisfied with the imaging provided, and although everything looks fine with the heart, he wants to review additional images of the cardiac arteries. He is certain that the information has already been gathered; he simply needs to locate it. As he indicates, “We haven't seen anything concerning here, but I'm not satisfied that we've got all the information we should get out of the scan...Unfortunately, the images I've got here are not diagnostic." To the researcher's surprise, based on both primary and secondary information, $\mathrm{PH}$ B then says: "Typically what I do is usually, at least where I work, I would sit down with the radiologist and we go over it together. That's the way I was trained to go over these tests. So 
that's generally what I feel. You know, two minds are better than one if we've gone over it that way." It is worth noting that PH B trained in Australia, rather than Canada. When the need for additional images not included in the file is raised again later in the interview, PH B says, "It's just the way I'm trained. I'm a bit of a stickler to go back and look at all the things myself...and I don't trust when anyone else tells me, unfortunately." SP B's response to this assertion is to say, "I find that reassuring" and "I really appreciate that you're being very honest with me. You want to take a closer look. I can appreciate that." PH B's sense of responsibility to his patients is apparent and clearly reassuring.

Although PH B is a Cardiologist, he also tells the patient that the spots on some of the images do not indicate cancer. In the role, Skyler's father died of lung cancer, so PH B feels it is appropriate to put the patient's mind at ease about this imaging finding. "I just don't want you to go home thinking he's seen a spot on my lungs; he's worried about lung cancer. That's what he's not telling me. No, that's not what I'm thinking at the moment." PH B is able to look beyond his own area of specialization, put himself in the patient's position, and provide assurance to the patient. Additionally, PH B offers to write down the name of the finding, lipomatous hypertrophy, so that the patient can "look it up on the web." He continues:

The only thing I can recommend on the web, if you want to look these things up on the web, let me recommend a website...There's lots of junk out there, and you'll get people writing all sorts of stuff. But generally I like to recommend one of the big sites: Canadian Cardiovascular Society, The American Heart Association, where we've put in input. We know that, we know it's the real, right information for you. They have excellent patient sheets, all that sort of stuff, so 
we can get you more information that way. So there are a lot of people on the web now and you're right to look it up.

Clearly PH B supports patient education, and fully understands patients wanting to self-educate. He also recognizes the potential confusion for patients reading misinformation online.

PH D's encounter with SP D as Skyler Hughes has a very different tone than PH B's encounter. During the encounter, and in the exit interview (see Chapter 8), PH D states that he has no experience, and limited knowledge, of the findings presented in Skyler's case; regardless, he chooses to continue his participation. In fairness, PH D is a generalist, not a specialist, and so it is possible that he might not have encountered similar findings in practice; however, the way he chooses to handle the situation shows questionable judgement.

Again, PH D begins by tossing aside the patient file. PH D admits in the opening, "I may be limited" and "We'll go through it together and I will do the best that I can with you." Throughout the interview, he repeats several times, "I'm limited in my knowledge of this kind of condition"... "I got to be honest with you, it's not my field." Towards the end of the interview, he states: "I've got to tell you, it's a very new condition. I've been a physician for thirty-five years, and it's the first time I've ever seen this." While it is responsible for him to admit his limitations, repeating it does little to comfort the patient, and nothing to normalize her situation. PH D begins reasonably well, given his limited knowledge for the case. He begins with the open question, "What brought you in to see Dr. Chan?" (Skyler's regular doctor) and listens to the patient's narrative, facilitating the story with the occasional "okay." Once the patient has delivered her narrative, PH D's next enquiry is to confirm that the patient is a non-smoker; this seems to pave the way for a less confrontational interview than the one with SP B. PH D then moves to more closed-ended questions: "Did he send you for a stress test?" When SP D says no, PH D asks the 
question a second time, somewhat incredulously. In retrospect, the researcher acknowledges that this information should have been included in the role, although it did not present an issue for $\mathrm{PH}$ B. When the patient indicates that she is not familiar with stress tests, PH D explains this test in understandable terms. When SP D confirms that the only investigations are the imaging, PH D responds, “That's all they did? They didn't do anything else?” PH D then asks more specific closed-ended questions: "Are you ever short of breath?" and "Do you notice any extra swelling of your hands or feet, especially your feet?" SP D says she sometimes gets short of breath when exercising, but has no edema (the swelling to which SP D is alluding), which might indicate cardiac insufficiency.

Having gathered that the patient is essentially asymptomatic, PH D states: "Your heart, for whatever reason, is getting insufficient blood flow..." He then tries to placate the patient by adding, "Well, it's workable. It's treatable." From here, PH D explains to the patient that the images show a thickening in the wall of the heart. Rather than admitting that he has no further knowledge, he starts guessing at what this might mean for the patient:

You've got a thickening of one of the walls. How much that's impacting on your heart situation, I'm not sure. We're going to need to talk to some specialists to see what's going on. So, you will be talking to a cardiovascular surgeon. That's my suggestion. I have a question in my mind about how much impact that is having and whether or not you might require cardiac surgery...But, in either event, we're talking, you've got to see a cardiologist, and possibly cardiac surgery... There's no question about this. You need to see a cardiovascular surgeon. You may or may not end up in surgery. My 
guess is you will; my guess is they probably take out this lipoma. It's

too large in that area...In any event, it is a consult with a

cardiovascular surgeon; there, there's no margin.

While it is clearly an appropriate recommendation that SP D sees a specialist, the tone and word choice make her condition seem extremely serious. PH D also makes it clear that the patient has no say in the matter; she will be sent to a specialist. SP D, who was told during training that this was a benign role, responds, "I wasn’t quite sure what, you know, exactly what my doctor was expecting to find from this." Note too that using the abbreviated "lipoma" (which is what appears on the chart) rather than the full name, "lipomatous hypertrophy," makes it sound closer to lymphoma - a cancer of the lymph system that is more commonly known than the benign condition of "lipomatous hypertrophy." This increases the risk of patient confusion and increased anxiety about her condition. Throughout the interview, PH D comments on this condition as being "unusual" and "very complicated."

From suggesting the patient must consult with either a cardiologist or cardiac surgeon, about 4 minutes into the interview, PH D continues:

And the mortality rates from surgery are very low. And, you must know people who have gone through cardiovascular surgery. So, it's not, “Oh, my God!” It's not pleasant. It's serious. I won't belittle it or anything.

Having admitted that he is unfamiliar with this condition, and that the patient should consult a specialist, PH D assumes that this condition will require surgery-but the chances are good that the patient will survive. Additionally, he assumes that the patient knows people who have had cardiac surgery. PH D refers to specialists several more times during the interview: “...once I've 
talked to the cardiovascular surgeon, I'll be in a far better situation to say, 'this is what we're going to do.' For now, I think, the first call is to get you the cardiovascular surgeon. Probably a cardiologist as well. They work together."

PH D also demeans his colleagues multiple times during this interview. Rather than suggesting that he might consult with the patient's regular doctor, he says: "Well, to be perfectly honest, had you come to me the first time, you probably wouldn't have gone straight through to this one [imaging]. I would've probably started with the stress test." Such statements have the potential impact of undermining the patient's confidence in her regular doctor. In addition to returning to the stress test several times, PH D also suggests doing a complete physical, including blood pressure. SP D had visited her doctor for a routine physical just three weeks earlier. The chart indicates that the patient's blood pressure is a reasonable 120/80. PH D also says, "I'm just going to talk to the radiologist. Just chat with him. I'd like to get his take on what we've seen. That's what they get paid for. That's why they make the big bucks." The provided chart includes the radiologist's findings for these images, quoted directly from the medical education website. While there is sometimes tension between radiologists and physicians regarding income levels for practice type, it seems wholly inappropriate and unprofessional for PH D to make such a flippant comment to a patient. Additionally, PH D is judgemental about the communicative abilities of the proposed cardiovascular surgeon, saying, "I might be better at explaining it to you, what's going on, once I find out." In this single encounter, with a new patient, PH D undermines the authority and professionalism of three other physicians, two of them highly trained specialists. This is demeaning to physicians, and it also potentially reduces the patient's confidence in those to whom she is expected to entrust her care. 
Rather than leaving the patient on a positive note, as did PH B, PH D's parting summary to the patient is:

Well, we want to make sure that when we go in [for surgery], we end up telling you that we're bang on. So, if you're already starting with this symptom, you know it's going to get worse. Everything in our bodies does. We've got to head this off. We've got to know what's going on. We've got to look into it. We've got to solve it. We've gotten pretty good at it. Cardiac surgery is much, much better. I'd like to talk to the radiologist. We're going to set you up with a cardiovascular surgeon. There's no question about that. You're going to see a cardiologist. That's a done deal.

This encounter does not reflect a patient-centred interview; the physician tells the patient what will happen to her body, as if she has no choice. In the process, he succeeds in frightening the patient; for much of this interview, SP D is verbally and physically shut down. Additionally, he makes the assertion that bodies, left to their own devices, necessarily become sicker; in his view, bodies are incapable of healing without medical intervention. In fairness, PH D does ask at about 9 minutes whether the patient has family or social supports; during this interview, he does not use this information to manipulate the patient.

PH B's primary focus throughout the encounter is SP B, although he frequently shifts back and forth between the patient and the images. PH D's focus is primarily on the patient; he seems to avoid the images. About halfway through this interview, the patient uses her prompt, requesting to view the images. Initially, PH D views them onscreen, but he then moves to the hard copies in the file. 
As with his previous interview, PH B uses medical language, but immediately defines it in understandable terms. Prior to sharing the images with SP B, PH B explains, "basically, a CT scan is a fancy X-ray.” He then explains why it might be useful in this case, before explaining the imaging findings. He consistently offers explanations, and answers the patient's questions. Conversely, PH D often makes assumptions about the patient's understanding, and does not adjust his language to the patient's level of understanding. For instance, as already noted, the patient must ask what PH D means by a stress test. Later in the interview, PH D catches himself saying, "What you're seeing here is an incursion," and immediately defines it as, "a pushing in of that area." While he simplifies his language, "incursion" is not a medical term, but common language. When beginning to explain the problem, PH D attempts to explain, "The heart is composed of four chambers; it [blood] goes through, goes into the other, goes through the lungs, back into one and then into the other." This is accompanied by hand gestures. This rapid-fire explanation would be a fine review for a medical student, or someone already familiar with heart function, but hardly seems adequate for a patient with no previous personal or familial cardiac history. Similarly, PH D uses the medical term "lipoma," indicating it is serious and will require surgery, without explaining what it is to the patient. PH D introduces the word, prompting the patient to ask, “So, it’s a lipoma?” Only once prompted does PH D respond: “Lipoma? I'm sorry, I know I should explain that." He then defines it in understandable terms as a, "fat cyst," "fatty tissue." He even continues saying, “one in ten people will have them, usually under the skin. They can be anywhere. It's benign. It's not a malignant process. It's not like its growing and causing all sorts of problems." His concern about the "lipomatous hypertrophy" is its location: "You only have so much room in the heart... so if it's encroaching enough, you're not going to 
get the blood flow in there, and we have to take that out." This lengthier and helpful explanation is offered only after the patient requests the information.

About 4:32 from the end of the interview, PH B asks, "Now, any questions about this test?" PH D does not make his patient a similar offer. PH B clearly answers any questions the patient has throughout the interview, and he makes the effort to ensure patient understanding. When PH D says that the patient will have to see a cardiologist and cardiac surgeon, the patient responds, "That's serious." To this tacit request for better understanding of the finding's implications, PH D answers, "Well, yes and no." His response is flippant, rather than informative or comforting to the patient. Fortunately, PH D continues and does explain and clarify in a little more detail.

Changes in body language are also noticed in both interviews. PH B is a very animated individual, who presents as someone with a great deal of energy. As a consequence, he uses many hand gestures. For instance, when asking the patient to describe the location of the chest pain, PH B motions towards his own chest. At 4:21 into the interview, PH B turns somewhat towards the screen to begin sharing the images with the patient. The patient similarly leans closer to the screen about 9 seconds later, and does not lean back into her chair for 4:20 minutes. Nearly two minutes into the explanation, SP B gestures towards the screen, asking, "So what are those flecks on the lung?" After leaning back in her chair for 3:49 minutes, SP B again leans forward for about 8 seconds, again asking about the white flecks, leans back again for 6 seconds, then forward again for another 2 seconds. Perhaps in response to the physician's activity, the patient also seems more physically active in this encounter than in her previous interview. After reviewing the imaging findings, while SP B's body is still turned somewhat to the monitor, PH B 
turns to look at the patient directly while emphasizing that these findings are nothing to worry about.

Although using the same monitor set-up, PH D experiences some frustration with both the hard copies and computer images. At 8:05 into the encounter, SP D pulls her chair closer to the physician to view the offered hard copy images. PH D responds by pulling his chair closer to her, so they are sitting beside each other. After 1:30 minutes, PH D leans towards the monitor expressing a need to find more images; note that physicians were informed that the images in the file and onscreen are the same. PH D moves his chair away from the patient, towards the monitor 14 seconds later. After struggling with the computer for 32 seconds to locate the desired image, he says sarcastically, "Don't you love computers?" At this point, the patient pulls her chair closer to the monitor too. After only 42 seconds of looking at the computer images, PH D pulls his chair away from the computer, and resumes eye contact with the patient. In both interviews, the primary focus is on the patient, except when viewing the images. PH D's focus on the images is more consistent, while PH B tends to move rapidly between the images and patient.

Outward signs of confusion or agitation are not observed in SP B during the interview. Conversely, SP D keeps her arms crossed in a closed posture throughout the interview. Although subtle, this slight agitation may be the result of unexpectedly being given bad news by the physician, when the role is anticipated to be fairly benign. In both cases, the patient's gaze remains on the physician, unless they are both focussed on the images.

PH B's encounter with SP B lasts 18:29, while PH D's encounter with SP D is slightly shorter, at 15:43. PH B only refers the patient to the screen, not the hard copy images. After reviewing the file for about 68 seconds, he moves to the screen 3:39 minutes into the interview; however, as previously noted, he tends to switch rapidly between the images and the patient, 
rather than focussing on the file, or onscreen images, for a long period. For instance, after spending 18 seconds focusing on the screen, he turns to the patient to indicate cross-sectioning on his own body, before returning to the screen 4 seconds later. After another 4 seconds of screen time, he returns to look at the patient for another 15 seconds. This rapid switching of focus continues for a total of 4:29 minutes of screen time, with the longest prolonged period of screen time being only 52 seconds, less than a minute. In this manner, PH B is assured of patient understanding, and meaningfully integrates the image into the interview. Later, PH B refers to the file folder for a total of 23 seconds, broken into five short-duration glances. SP B's viewing is less broken up than the physician's. She spends 2:25 focussing exclusively on the images, even when PH B looks at her. Later in the interview, she spends a total of 43 more seconds on the images, broken into four viewing periods.

PH D uses the images quite differently in his encounter with SP D. He turns to the monitor at 3:22 minutes into the interview; however, after struggling for a few seconds, he quits and returns to the conversation. Another 4:30 minutes later, SP D uses the role's prompt requesting to view the images. Once prompted, PH D picks up the file of hard copy images. After reviewing these with the patient for 1:25 minutes, he returns to the monitor, seeking more images. After another 1:14 minutes focussing on the screen, he returns to the conversation. While PH D is only focussed on the images for 2:39 minutes, SP D maintains her focus for 2:58 minutes, with only one glance at the physician.

\section{$\underline{\text { Chris Anderson }}$}

The fourth and final role is that of Chris Anderson, age 72, whose recent diagnosis of high cholesterol and high blood pressure has responded well to medication over the last six-months. 
In the last three weeks, however, s/he has experienced increasingly recurrent attacks of acute chest pain, accompanied by breathlessness and sometimes referred pain to the left arm or jaw. After seeing his/her regular doctor, s/he was sent to a cardiologist, and then had a frightening angiogram experience. Chris has a strong family history of cardiac problems, and is a takecharge A-type personality who wants the problem fixed. (See Appendix D for complete role, chart notes, and images.) This role was encountered by PH B (Cardiologist) and PH C (GP).

PH B's encounter with SP C as Chris Anderson begins with the Cardiologist clarifying a few points, such as: "You've been having some chest discomfort?...When does this tend to happen?...Was it getting worse?" The patient's narrative is facilitated by the physician saying "okay" at intervals. Once PH B has a clear understanding of the problem, he then clarifies the patient's prescription medication use, including, "How often you taking the [nitroglycerin] spray?" 119 With the A-type patient pushing somewhat, PH B proceeds to the imaging; however, he first explains, "The angiogram is sort of a gold-standard test; it really tells us exactly what's going on with the arteries." Having correctly read this patient's personality, PH B selects a specific image, and maintaining direct eye contact with the patient, says, "We'll go over that one first, because it actually shows there's quite a number of narrowings in the arteries to your heart. So, there's no doubt that this is the cause for the chest pain you've been having." Having delivered the bad news, PH B carefully describes the heart's anatomy, using hand gestures to explain its structure, aided by the images. He also explains why narrowings of the heart's arteries are problematic. This is followed by a clear and simple explanation about the condition of the patient's heart; this is the good news, after having delivered the bad news. PH B then delivers three treatment options: 1) do nothing, 2) add more medication, or 3) surgical intervention. He offers the most detail on the option of a surgical bypass, saying both, "It's a big operation" and

\footnotetext{
${ }^{119}$ Nitroglycerin is used as required by cardiac patients to rapidly relieve angina symptoms, such as chest tightness.
} 
"It's the most commonly done operation in the world today, so we have a lot of experience with it." Following this, PH B details another surgical option: an angioplasty to open the arteries and introduce stents to keep them open. To illustrate the appearance of a stent to SP C, PH B unscrews his ballpoint pen and removes the spring, saying, "It actually looks like this. It comes in a collapsed form. We put it in the artery, we expand it up. It's stuck in the artery forever, but it keeps the artery open."

PH B acknowledges, "I've given you a lot of information," and suggests that he provide SP C with some reading material on each option so that he can make an informed decision. PH B acknowledges, "There are pros and cons for each particular treatment strategy.” After answering SP C's questions, and offering the advantages and disadvantages of each option for this patient, PH B declares, "My overall recommendation would be that I believe bypass surgery would be the best treatment for you with the plan of - that will take away your angina, keep you out of hospital, keep you alive longer." While the A-personality patient is eager to agree to the surgery, PH B slows him down, saying that he will write a prescription for additional medication to help him over the short-term, and suggesting that SP C speak to his regular Cardiologist, and a Cardiac Surgeon, as well as involving family members, before making his final decision. PH B then summarizes what has been covered during the appointment, encouraging the patient to think critically about the options presented. As well as assigning the patient homework, PH B says, "Okay, so what I will do, as I said, my homework, I would normally go back and just look at the total raw images to make sure, confirm everything." PH B also goes over emergency contingencies: what to do if the pain escalates or fails to respond to the nitroglycerin spray. He also encourages the patient, when consulting with the other doctors, to "Take a pen and a piece 
of paper. Write stuff down because you're going to forget it when someone starts telling you all these facts."

When SP C suggests that this encounter is very different from his imaging experience, saying, "I was scared, and then by the time I left, I was twice as scared," PH B diplomatically responds, "I can't speak for the guys who did the scan, but generally they don't want to give the information because they don't know the whole story. So, they don't want to say something without knowing your history, your symptoms and, I think, tell you the wrong thing." Having explained why the imaging team might be reticent, PH B continues, "So maybe we should go back and discuss it with them, because they should give you some, you know, at least some reading or something about it. But that's - I can't speak specifically for them." Towards the end of the encounter, SP C says, "I'm really relieved to know that at least I have an option to do, because I was told I had no, I thought, I'm going to die, and I felt really badly when I left [the imaging appointment]. But as of today, I'm buoyed, I must say.” Clearly, PH B's handling of this interview gave the patient hope and a positive outlook on the upcoming challenges.

PH C's Chris Anderson encounter is also with SP C and begins similarly with the physician inviting the patient's narrative, with questions like: "How have you been feeling?... Any more chest pains?...How's the medication working?... Any trip to the emergency or the hospital since the last time you were here?" With this information gathered, PH C proceeds to inform SP C of the imaging results, saying that the news is "good and bad." The good news is that the heart is healthy; the bad news is that some arteries show significant narrowing. The good news is emphasized through repetition towards the end of the interview. About halfway through the interview, at 9:37 minutes, PH C suggests two options: a bypass or an angioplasty. In simple language, PH C explains each procedure, but the patient feels compelled to prompt, "Are there 
advantages and disadvantages to both?" Once prompted, PH C suggests, "There's good and bad for each. The angioplasty, there may be a little bit of higher risk of re-stenosis; it may get narrower a little bit faster than the bypass, but the bypass, like I said, it is a major operation." Ultimately, he defers to the Cardiologist to make the best decision for this particular patient. PH $\mathrm{C}$ also reiterates the importance of the patient's medication compliance to "help keep the arteries unclogged, hopefully as long as possible," adding that the patient should be careful of physical, as well as emotional, over-exertion, and always keep the nitroglycerin spray readily available. The need for adherence to the medications is repeated at the end of the interview, with the addition, "If it [nitroglycerin spray] doesn't work, or you find it [chest pain] persists, then come to the hospital, and then we'll take care of you here." PH C also checks to see who lives with the patient, and advises SP C to apprise his wife of his medications and his condition.

When the A-personality patient starts pushing to schedule the surgery, PH C suggests that the next steps will be to see his regular Cardiologist, as well as a Cardiac Surgeon. When SP C alludes to his anxiety during imaging, PH C does not follow up; however, the patient continues, saying, "I'm glad to hear it [the imaging results] from you." The SP's response indicates some sense of relief at the conclusion of this interview.

Once the opening patient narrative is delivered, PH B clearly dominates the interview with SP C. Although the patient does ask some questions, he has to interrupt the physician to do so. PH B sometimes uses medical terminology; however, it is consistently and immediately defined in simpler terms. At one point, he states: "So, as Cardiologists, we're plumbers. What we do is we have a look and see, are the pipes blocked off, or are they open? And if they are blocked off, what's the best way to fix it?" This simple explanation prompts the patient to respond, "I never thought of it that way...That's easy to understand." Similarly, when PH B explains that the 
heart is getting adequate blood supply, he refers to an image and asks the patient, "You see it looks sort of like a doughnut?" When the patient acknowledges that he does, PH B continues, "Okay, doughnut's good, because what that means is you're getting enough blood through." When PH B explains that the "heart muscle is a little bit dilated" and the patient guesses, "Dilated is narrow?" PH B refines this definition, saying, "It's starting to swell up... the heart muscles are getting narrower.” Although PH B does not regularly clarify the patient's understanding, he is open to answering questions, acknowledges the amount of information he has delivered, and encourages the patient to write down questions or take notes during appointments. Only near the conclusion, he asks, "Any questions about any of this?"

Well into the interview, after the findings and treatment options have been explained, SP C asks, "Is my life in danger right now?" PH B acknowledges this with:

Okay, good question. So, what do we know about this? If we did nothing and just kept you on your tablets, it is likely that these narrowings will continue to, over time, get worse and you could have a heart attack... Now, good medication therapy is effective, but we know it's not as effective in preventing heart attack, stroke, death, as, it's not as good as the surgery, or the balloon-type treatment option.

PH B's experience and knowledge are evident throughout this interview; he even offers evidence from clinical studies regarding the efficacy of bypass versus angioplasty options.

PH C's encounter with this patient is more conversational than PH B's. As well as openended questions at the beginning of the interview, PH C consistently checks the patient's understanding. Most of his sentences ended with, "Okay?" This simple addition invites the patient to ask a question if he has one. Throughout the interview, the physician's focus is almost 
always on the patient, other than when he is explaining an image. PH C almost consistently uses lay language, or immediately explains medical terminology. For instance, at one point he says, "myocardial profusion defects." He immediately turns to the patient, and expanding on what he has said, states: "That means that parts of the heart that kind of light up when they take the images...if there were certain areas where, let's say, blood vessels were completely blocked, and the muscle's starting to die in the heart, that would show up differently from the healthy heart muscles." When PH C fails to define what a stent is, and SP C asks, PH C struggles with his definition: "A stent is something that, it's kind of like a, how do you explain it? It's kind of like a, it's a metal piece that, sort of, keeps the artery open. It kind of opens up, and it [stent] keeps it [artery] open." Although PH C has to work at it, the patient seems satisfied with this explanation. When explaining the meaning of the results, $\mathrm{PH} \mathrm{C}$ also explains the impact on the patient: They said that there's a more than $90 \%$ stenosis, okay? That means that there's a narrowing. It's not blocked off, but there is a narrowing. And, what happens is that, basically, when you feel the chest pain, is that when there is narrowing in your arteries, especially when you exert yourself-you walk, you go up a flight of stairs, you walk a block or two, whatever - the heart pumps harder, and the blood has difficulty going through because it's [arteries] narrow. And that's how you end up with the pain in your chest. And once you take the spray, the nitroglycerin, that sort of relieves the spasm.

Throughout this explanation, the patient says things like "Yes" and "Exactly," acknowledging that the physician is understanding his illness experience. 
When SP C uses his prompt requesting to see the images, PH C shows him selected images, but also indicates, "I was going to show it to you, but then I thought it might be difficult to see." He explains, "they [the images] show you the arteries, and they show you some narrowing, but it is very difficult, because it's not like a, sort of, complete image of the heart. This is just part of it." Similarly, the patient has to prompt the physician regarding post-surgical recovery and what kind of health improvement might be expected. Although PH C is able to answer these questions, the patient has to ask, as such information is not immediately offered.

As with his previous interview, PH B's gaze moves rapidly between the patient and the images, remaining largely on the patient. Importantly, he uses strong eye contact when explaining the impact of the diagnosis, and the treatment options. Additionally, he uses verbal underscoring with phrases like, "This is important," drawing the listener's attention to the information that follows. At the opening of the interview, PH B spends about 17 seconds looking at the file, hands on knees, but once he begins to speak to the patient in earnest, he sits back in the chair, and when he is ready to look at the images, he gestures towards the monitor. Once opening clarifications are complete, at 2:48 minutes, PH B opts to use the hard copy images. To facilitate discussion, he leans towards the patient — away from the monitor — and uses hand gestures. After a further 12 seconds, he turns to the monitor and brings up the angiogram, expressing his concern about the image's small size. The patient appears somewhat anxious at the beginning of the encounter, as is appropriate for this role. When the diagnosis is delivered at 5:05 minutes, the patient suddenly sits back into his chair, physically expressing shock; however, he seems quite relaxed and calm by the end of the interview. As noted in his previous encounter, PH B is very animated throughout the interview; his gaze shifts rapidly between the patient, the hard copy images, and the monitor. PH B shifts in his chair a great deal, but never turns his back 
on the patient in favour of the image. Also, as previously noted, PH B uses hand gestures to good effect. At 5:35 minutes, he masterfully uses hand gestures to describe the three main arteries of the heart. He also gestures to his own chest on four occasions throughout the interview when describing the problem and the treatment options. Encouraged by the physician, at one point, SP $\mathrm{C}$ leans towards the monitor for about 5 seconds; however, his gaze remains almost exclusively on the physician.

PH C's gaze is similarly focussed almost exclusively on the patient. PH C increasingly uses hand gestures through the interview. About one minute into the explanation of findings, hand gestures are used to emphasize the narrowing of arteries and the effect on the heart. Hand gestures are also used to help explain the treatment options. While SP C appears somewhat anxious at the beginning of the encounter, as is appropriate for this role, he seems increasingly calmer as the situation is explained. Again, the patient's gaze remains almost exclusively on the physician.

The difference in overall duration between these two interviews is the most extreme during this field research. PH B's interview is 20:15 minutes, compared to PH C's at 10:08 minutes - almost exactly half the time. One explanation for this discrepancy might be that PH B is a Cardiologist with more detailed information to offer the patient, than a generalist like PH C. It should be noted that in spite of the time discrepancy, the patient seems equally satisfied by both interviews.

In total, PH B spends 32 seconds reviewing hard copy images, divided into four segments, mostly towards the end of the encounter when discussing surgical options. Additionally, he spends just over a minute, 63 seconds, viewing onscreen images, with two of the four segments being about half a minute long. The file is viewed for 38 seconds at the 
beginning of the interview, and only another 3 seconds later. During the first 4:35 minutes of the interview, another 46 seconds is spent in the file, but it is always broken up with glances at the patient. After this, PH B turns to the monitor. Demonstrative hand gestures amount to 27 seconds over six segments. The stent demonstration using the pen spring requires only 16 seconds, from unscrewing the pen to conclusion. As previously noted, the patient's gaze is almost always on the physician. During this interview, SP C spends 2 seconds looking at the written file; a further 5 seconds are spent on the file when discussing medications, with 2 of these seconds remaining on the file after the physician stops looking at it. Perhaps guided by the physician, the patient's gaze on the images tends to be broken up. In total, the patient looks at the screen for only 31 seconds over 9 glances, all under 10 seconds each. At 8:59, he points to the screen, and says “That's on there?" in reference to the physician's explanation that his heart is beginning to struggle. PH B refers this patient query to the hard copy images, rather than to the monitor.

Similarly, PH C's focus remains largely on the patient. In total, he spends 38 seconds on the file over five segments, the longest being 33 seconds during his explanation of findings about a minute into the interview. Viewing the images begins at 2:24 minutes into the interview, and requires only 16 seconds on two hard copy images, with one quick glance at the patient when the patient is not looking. When struggling with how to define a stent, PH C looks away into space for 3 seconds. The patient's gaze remains mostly on the physician. Prior to PH C entering the room, the patient views the image onscreen, but no other use is made of the monitor. When SP C's attention is drawn to an image by the physician, the patient engages with the images for those few seconds. 


\section{Conclusion}

The researcher must acknowledge that one of the limitations of this study is the low number of physicians recruited to participate. Four is clearly too small a sample from which to extrapolate larger findings. Careful observation does, however, indicate some trends worth considering.

To varying degrees, PHs A, B, and C all exhibit patient-centred communication techniques during their interviews. Conversely, PH D, the most senior physician in this study, displays a more biomedical or physician-centred communicative approach. One might conclude that changes in medical education over the last three decades to incorporate patient-centred communicative training have resulted in positive changes in the manner in which physicians engage with their patients. Without addressing similar research to a larger participant body, of similar demographic breadth, it is impossible to know whether the difference in communicative strategy is due to changes in training over time, or simply a difference in individual style. Both specialists, PHs A and B, exhibit strong communication skills, as does PH C, a generalist.

The time focussed on the images has little bearing on the interview's communicative success, or SP satisfaction. Consistently, physicians focus primarily on their patients, using the images only as tools for reference and to aid their explanation of findings. PH D, who spends the least amount of time on the images, exhibits the poorest communicative skills during these encounters.

Although the time spent reviewing images with patients is minimal during each interview, in all cases, patients and physicians focus on the images for some part of the interview. What is notable is that in all cases, the patients view the images as they are, either overtly or tacitly, directed to view the images by their physicians. For instance, during their interviews with $\mathrm{PH} \mathrm{B}$, who tends to glance rapidly between the image and patient, both SPs tend 
to mimic this viewing pattern, which is different from their viewing patterns during other interviews. Both patients and physicians are observed viewing the images when they are alone. Images have power in the room.

The following chapter discusses the results of post-encounter interviews with the physicians and SPs immediately after their encounters. 


\section{Chapter 8:}

\section{Simulated Patient and Physician Perceptions of Encounters}

The good physician treats the disease but the great physician treats the patient who has the disease.

\section{Dr. William Osler (1849-1919)}

The previous chapter offers an analysis of the encounters between simulated patients (SPs) and physicians. This chapter analyzes the exit interviews with both SPs and physicians. Once physicians had engaged with two SPs, their session ended with a one-on-one semi-structured exit interview (see Appendix F). Interview questions were designed to provide an opportunity for each physician to reflect on his performance during the interviews, consider the relationship established with the patient, as well as allowing the researcher to check if the physician thought that he had performed as he normally would during a patient interview. Once the physician interviews were complete, each SP who had portrayed a role with that physician was also individually interviewed. As the focus of this research is communication, and these SPs are experienced educators in the medical communication context, their feedback adds another critical dimension. SP feedback was usually similar to the researcher's observational assessment of the role play, thus adding credibility to the analysis. This chapter is organized by physician, in alphabetical order (A, B, C, and D), with the SP interviews for each physician included in each section.

\section{Analysis of Exit Interviews}

I think he was trying to explain to me what the images meant. It was like he was the navigator and he was showing me the maps.

SP A regarding PH A encounter 


\section{$\underline{\mathrm{PH}} \mathrm{A}$}

$\mathrm{PH} \mathrm{A}$ is a radiation Oncologist in the GTA with eighteen years of professional practice at the time of this research. He specifically requested additional SP feedback during his exit interview, and both SPs obliged. PH A declares that he is happy with both interviews and that he has said and done the things he would in practise; however, he adds that normally he would order blood work and perhaps other tests prior to this patient encounter. In terms of communication, he comments, "I tried not to use too many big words, and I also usually I try not to use the word cancer. I try to use the word growth, because sometimes people don't like the word cancer." When asked if he feels that he offered the patients adequate opportunities to ask questions, his response is:

I usually ask them [patients] at the end of my conversation, do they have any questions. I try, and it may just be my way of, the so-called, train-of-thought, right? Say, I usually try to, once I start talking, I keep talking, and sometimes I should try...I know and I try to stop and ask them, do they have any questions and things like that. But sometimes I feel that if I do this, sometimes I've lost my train-of-thought, and therefore it is a little bit more difficult for me to go back and start to continue my conversation and things like that, so, yeah, so sometimes I'm still struggling about it. Say, if it is a simple case then it will be fine, but if it is a little more complicated or whatever then it may be a little bit, sometimes difficult.

PH A is aware that sometimes he may overwhelm patients, but he works consciously not to. His response clearly shows the difficulty of trying to facilitate two complex tasks simultaneously: 
analyzing complex data, while also communicating effectively in a challenging situation. When asked where he thought his attention was generally focussed, his response offers an interesting insight into the way this physician uses imaging.

I usually like to do, is that I try, is to show, show that patient, for example, for lung cancer, is to show them the image, because it will be, it explains a lot if they can see the image and see exactly what is there and all that. And then sometimes it may not be-it may be a little bit too overwhelming for some of the patients if you show them too much. So, it is like a balance. You just need to show them what is. One, what is considered to be enough, and then it depends on the patient's reaction.

Here again is evidence that $\mathrm{PH}$ A recognizes the difficulty in communicating with patients, and that he, as the physician, always has to balance what is adequate information for each individual patient, given the patient's reaction.

When PH A encounters Lee Berkowitz (benign), he only shares two images with the patient, SP B, although others are available. When asked whether this decision is based on his assessment of the individual patient, PH A responds that he just showed a "representative image," because in her case, the growth is benign and he did not wish to alarm the patient unnecessarily. When he encounters Dana Jones (findings), however, the mass is significant and cancerous. In this case, PH A shows SP A many images to assure him that although there is a substantial mass, and a serious health concern, it is localized and has not metastasized into surrounding organs. 
Although PH A delivers what seems to the observer to be two very patient-centred interviews, when asked, "Are you familiar with the philosophy of patient-centred care, patientcentred communication?" He responds, "Not really." As with many things in life, it is possible to practise something without being aware of the theory, or having forgotten the theory.

Although PH A worked with the room set-up as it was established, he also says that normally he would move the monitor closer, "in front of both the patient and myself." He also says he prefers to be on the other side of the patient, easing access to the mouse and computer.

When asked about the amount of eye contact with his patients, PH A responds, "I try to. I always do not have. I don't know. It is important, I know, to have good eye contact with the patient. Yes. But I don't think I have enough.” He expresses that he felt a little disorganized during the Lee Berkowitz encounter, in part because this is not a case that would normally be referred to him; such cases would be referred to a thoracic surgeon. He also agrees that while the "gold standard is going to be a biopsy," in a low-risk case, the potential risk of performing a biopsy would be difficult to justify. His recommendation would be "to repeat the scan in a few months' time to observe it."

When SP B is asked about her encounter with PH A, she answers, "I thought it went really well...I thought it was fairly conversational...I thought he was very thorough.” She also expresses that the discussion was longer and more detailed than she had expected, saying, "I was impressed by that...he was really listening to me..." Regarding the communication, she declares, "I understood everything he was talking about," including descriptions beyond what was viewable on the CT scan. While commenting that not much empathy was apparent, she also says, "I felt that he was concerned," adding, "he didn't coddle me in that way, which perhaps that was good." With regard to the amount of eye contact, something PH A expressed concern about, SP 
B responds, "I thought the eye contact was just fine." Additionally, she notes that PH A often nodded his head to facilitate discussion. SP B reports that she did not find the images distracting during the interview, although she thinks that she referred to them more than did the physician. She adds, "I liked the fact that he showed the X-ray, and then went down and showed the CAT scan, and how clear it was. It's such a clear sort of image.”

Overall, SP B is very complimentary about PH A's performance, saying that she found him "reassuring." She ends her interview by saying:

I mean, I really felt that the relationship with this doctor was certainly not over, because you've got to keep....and that was nice. It wasn't dismissed as, oh this is definitely benign. We're just going to... I felt the relationship, it was definitely implied that we were going to be having contact, and looking into this, and this was just the beginning of the process. So, I wasn't being dismissed as just, oh, we don't need to look into anything further... If that had been me, and if that had been my doctor, I would have been pleased.

SP A's exit interview regarding PH A is somewhat more critical than SP B's. This might be due, in part, to the anxiety caused by playing a role in which one is told that there are findings. Although SP A expresses that, "I felt the encounter went well," he also says, "I felt a bit overwhelmed and scared." Reflecting on the encounter's conclusion, he admits, "I didn't leave feeling scared. I mean, obviously, I did leave scared in that I knew it was cancer, but I thought he approached that incredibly well, in that he was very honest about it and up front." Later, SP A says, "There were a few times where I felt scared and I think he addressed them." 
Regarding the communication, SP A comments that some medical jargon slipped in once or twice, but he always asked for clarification. SP A also comments, "It took him a while to get to the bad news, but I think he did a pretty good job, and I really did appreciate his kind of upbeat attitude. It wasn't negative or dour, and I thought that was good." He expresses some relief that PH A, "didn't dwell on the risk factors," such as the strong family history of cancer, or the patient's smoking history. Instead, "I think he stayed with the problem at hand, which was the growth in my lungs, you know? So I didn't feel judged. I didn't feel like I was an idiot for smoking so long." Although the patient commented on his smoking history during the encounter, it garnered no reaction from $\mathrm{PH}$ A.

When asked whether the encounter was conversational, SP A responds, "I definitely felt like it was generally a counsel as opposed to a conversation, but it didn't feel like I was excluded from it. It didn't feel like he was preaching to me." While expressing this, SP A also indicates that he often felt overwhelmed by the information; because PH A did not routinely check in to ensure understanding, or see how SP A was responding to news of the diagnosis, the patient became increasingly overwhelmed. As SP A expresses it: "Because it was a lot of information. And at the end of the interview, he said, 'Do you have any questions? I know I'm giving you a lot of information.' And, you know, at that point, it's already too late... I would be overwhelmed and not know what to say."

While the images were very present for SP A in the encounter, it "wasn't all about the pictures," and he did feel engaged "in a very nice way." From the researcher's perspective, the eye contact seemed strong during this interview; however, as the patient engaged with PH A, SP A reveals, "I thought there were times where he, it seemed like he was focussing on my shirt, as opposed to my face." SP A acknowledges that this trend improved through the interview, and 
stronger eye contact was provided when PH A discussed the images, and towards the interview's end.

When the interview ended, SP A says he felt reassured that there was a team behind this doctor, and that "we were talking about the future." Ultimately, SP A reports, "he made me feel like he was there with me. It wasn't like I was just another patient.”

\section{$\underline{\mathrm{PH} B}$}

Physician B is a Cardiologist in Toronto with thirteen years of professional practice at the time of this research. When asked for a general assessment of his interviews, he responds, "Fine. Fairly standard," adding that the room set-up is similar to his own office. "I would have all the information there on the computer in front of me." Later, he adds, "Although I generally try and keep the computer a little bit behind me, or we have a monitor that I move around so that when I want to show something, like a diabetes check, there it is. And then I put it away, because otherwise, as I said, everything tends to focus on that." PH B's comment indicates his awareness of the possibility that imaging, or other information on a computer monitor, can dominate patient interviews. While the interviews were similar to his normal style, he adds, "Generally, if it was the first time, I would take a bit more of a thorough history and exam."

Regarding patient communication, PH B comments, “They both seem fairly intelligent and understanding...Do what I usually do; try to repeat things a couple of times. They get the take-home message." When asked about his suggestion that patients write down their questions, and let him advise them on appropriate websites for information regarding their conditions, PH B comments that, especially in bad news situations, "I find people just can't remember anything... if you can, give them reading." He cites that another potential problem in delivering bad news is, 
"they [patients] misinterpret something you say and then that becomes the key event." Even when a physician is communicating consciously, he still may not be successful; this is one of the reasons why physicians must adjust to each individual patient's communicative needs. PH B also acknowledges that in a busy clinic, sometimes appropriate materials are not available to give patients. "So that's the ideal [offering pamphlets], but it doesn't always work." He also adds, "I mean, that's why generally if I'm going to do a treatment on someone, then we'll ask them to come back with a family member. If it's something I'm less concerned about, then I won't generally do that." A family member or friend can offer patient support, but can also hear and record details of the patient-physician encounter somewhat more objectively than the patient whose body is under discussion.

PH B tries to focus his attention on the patient, but is acutely aware of the allure of technology: "Certainly the technology is nice and you do drift towards it because you've got these pretty pictures.” During the exit interview, he acknowledges, I often don't show the direct images because of that reason, because I think people just sort of interpret what they want out of them [images] as opposed to - they can't interpret them in the timeframe anyway. So I generally say, look, you know, this is what we showed. If you want an image, I can give you something, but I don't usually go through everything in great detail with the image because I just think it's information overload and it's too hard.

Although PH B might use images to facilitate his dialogue with patients, he carefully considers which images, how many, and what he thinks would be most beneficial for a particular patient. 
For instance, in one of his SP encounters, he showed only two of the available eight images. In his estimation, most patients won't "get it" if shown their images. He concedes that, The angiogram is a little bit easier if you have a good quality angiogram, and it goes from open to very narrowed, and open. Or if you have a big lump in the heart or something, it's easier to show that. But then more subtle degrees of pathology, which is what most people generally have, it just gets lost in translation.

Radiologists undergo months of training learning how to read medical images, so unless something is obviously wrong, it seems unlikely that most patients will appreciate a problem from the images alone. For instance, The Michener Institute for Applied Health Sciences, associated with both the University of Toronto and Laurentian University (Northern Ontario School of Medicine), offers a fulltime twenty-month ultrasound program and a nineteen-month part-time MRI program. Upon completion, students can apply for full degree status at an associated university, and fulfill medical sonography or ultrasound licensing requirements (Michener Institute). As PH B suggests, So, unless it's really obvious that a lay person could appreciate what they're looking at, it's counterproductive. I don't do it. If people ask for it, sure. But I find it takes a lot of extra time. People pick up on things. What's that? And this is a cancer, etcetera, which is fine. Then if I haven't got all the information, then it actually takes more time in the long term, because you have to go back and have a long discussion, so.... 
For example, during his encounter with SP B, the patient noticed some white specks on the images, which were not indicative of pathology, but caused a digression in the discussion into something unrelated to the presenting problem.

After doing two very patient-centred interviews, PH B's response regarding patientcentred interviewing is surprising. Although he is aware of the concept, he seems dismissive of it:

I understand the sort of terms like, you know, from my perspective just trying to be open and give the patients opportunity to ask questions, is a, you know, and try and convey the therapeutic options in the easiest possible light is an effective way to do it. At least that's my style. But I don't run through a specific checklist-I have to do this, and I have to do that. Yeah, but on the other hand, it's good if you do run a checklist, because then you don't rush and you don't forget it. But no, that's how I try and do it, but....

PH B seems to equate patient-centred communication with following a checklist; however, following a checklist is often perceived by patients as very clinical and linear - the opposite of patient-centred. Rather than adjusting to a specific patient's needs, the physician following a checklist may be perceived as insincere or simply going through the motions. In the simulated context, SPs sometimes joke about physicians who, at an emotionally difficult moment for the patient, say, "I'm sorry," and then immediately ask "Do you smoke?" During feedback, the SP might suggest that s/he felt a lack of empathy; invariably, the physician will counter defensively with, "But I said I was sorry," not understanding that saying something by rote hardly passes as empathy. When an expression of "empathy" is insincere, the physician will find it more difficult 
to create a sense of trust with a patient. Communication can become very stilted and closed, so the therapeutic alliance is not well formed.

PH B reveals that he has "tricks" for appearing patient-centred, while retaining his efficiency. For instance,

I find in terms of putting people at ease, you can actually save a lot of time by, sort of, appearing to be close and do a few things like shake their hand and sit down, and that people feel you're giving them a lot of time, even though you may be rushing things through. So, it's that sort of body language stuff. So, I don't like a big thing in between [myself and a patient, like a desk].

For him, appearing patient-centred is about creating a desirable perception, while not necessarily practising the concept. PH B strives to create, "the perception that you're actually putting in a lot more time, and touchy-feely sort of medicine stuff." As noted in Chapter 3, however, patientcentred medicine is often more efficient, not less, than a purely biomedical encounter; it does not necessarily require a more substantial temporal investment. "Like putting your coffee down" PH B continues. "If you walk into the room with a coffee, if you put it down, and then turn around and face someone, then they like, well, you've really got my attention now, because you've stopped what you're doing. And then you can pick it up and do whatever you want with it after that." Although PH B considers these "tricks" for appearing patient-centred, that he is somehow creating an illusion of being patient-centred, an argument could also be made that he is behaving in a patient-centred manner. Certainly body language is part of appearing to be patient-centred.

Regarding eye contact, PH B feels that his eye contact with patients tends to increase as an interview progresses. Additionally, he concedes that, 
...if I'm not quite as confident about what I should be doing, I think then I do less eye contact, because when you know this is definitely clear cut, this is easy—bang, bang, bang. Whereas when it's like, gee, this is a grey zone. I start to do this [eyes upward, thinking] a bit more, and there's a bit more sort of the body language comes out.

Finally, although PH B thinks the patients he encountered during research, "seemed reasonably happy," he acknowledges that while trying to give patients the time they need, "I think you can always do more, but then there's time constraints."

SP B, who encountered PH B as Skyler Hughes (benign), describes this interview as both "energetic" and "very clear," indicating that as Skyler, after this encounter, there "would not be a concern." She concedes that "he didn't look worried, but he definitely stuck by his decision that he needed to look further." SP B appreciates that PH B was, "very upbeat" and remained patient, even when Skyler asked questions obviously based on misunderstanding or limited knowledge (i.e., were the calcium deposits in the images caused by drinking too much milk). She found his use of language clear and accessible, and as a patient, she found him "approachable." SP B was comfortable asking questions, knowing that they would not be dismissed as "stupid." She comments that although the interview started in an open-ended manner, this was followed by PH B telling her of his concerns, while also expressing that she need not worry. For the patient, the encounter became less conversational as it progressed. She did, however, feel that she had adequate opportunities to ask questions, and says, "he was quite willing to stop and listen to my questions.” Ultimately, "I didn't leave worried, but things weren't concluded.” SP B also feels that, "this fellow was interested in me, not dismissive." 
SP B suggests that the eye contact was "very good" during her encounter with PH B. When asked whether the images were distracting, she responds that they did not adversely affect eye contact, adding, "I felt we needed them [the images]. We really needed them." When asked to expand on this comment, she says:

Well, I don't think I could've understood about the, sort of, separation in the heart, between the two areas in the heart, unless I could've seen it, because it's strange looking at the CT-the way, the slice of you, the perspective. It takes a few moments to, sort of, orient yourself to how you're looking at everything. But right away I noticed the specks on my own, really, and wondered what those were, because of the illumination.

Overall, SP B finds the images "very helpful" rather than distracting, although these white specks that caught her attention were something that PH B found unhelpful during the encounter. SP B appreciates that this physician was "forthcoming" with information and "very thorough." She comments several times on PH B's high energy, also reiterating that he is able to stop and listen to the patient's questions. SP B found it positive that PH B referred to her regular doctor, by name, several times during the encounter. Additionally, she appreciates the handshake at the interview's beginning; while PH B may view this action as a "trick," it clearly had the desired effect of creating an instant and positive connection with this patient.

PH B also encountered SP C as Chris Anderson (findings). When asked his overall impression of this interview, SP C states:

Well, from the beginning to the end, I thought it went fabulously. I thought he was forthright, honest, didn't pull any punches, told me the 
plain truth and that's exactly what I wanted to hear. I could feel myself being elevated in mood, and I could feel myself being buoyed and supported because of this guy's understanding, and seemed to have- he mentioned a couple of times about there's so much information here, he mentioned that a couple of times. So, the fact that he was explaining this to me, and he even said something that was not quite layman, and I mentioned something about it, and right away he told me what it was, and I was allowed to excuse myself and say, "Would you mind if I ask you this?" And, he was just more than patient and he let me know exactly what I needed to know, and I just felt he was a pretty honest fellow. He let me know it was a serious thing as well. So, you know, this is a serious thing and you better have your family member with you when you get in, because there's so much information that you want to know, everything you need to know. Then he gave me his opinion on what choice I could make, and what he would recommend. And, I felt so much different than when I went and got my original test. I felt left out [during the angiogram], and I was able to say that, and he was able to acknowledge it. And, I liked what he did though; he respected the people who did it, and I respected him for that. That he didn't — they're his colleagues. Things happen in these things [medical testing], and people are all different. And so, him letting me know that, made it all really good for me. 
Although Chris starts in a bleak place, SP C comments that PH B, "he didn't rise to the bait." Although this role provides opportunities for the SP to be very dark and to think the worst, SP C explains, "I thought I was armed with all kinds of things to throw, but I had no need to throw them." PH B's pleasant and positive manner effectively disarms this patient, creating a more satisfying interview for both parties. A strong therapeutic alliance was formed, so the patient felt, "I'm not the only one in here [dealing with the medical crisis]." Chris is written as a "control freak," but SP C ended the interview with the sense that, "I knew what I had to do, to do my part in making - I had a part to play in this, and he showed me what it was. We'll do our job, and left me to do my job." Clearly, PH B provided this patient with a sense of empowerment and control, in spite of the dire circumstances.

SP C found the language understandable, adding, "when he described himself as a plumber, my day was won." While SP C did not find the encounter conversational, he did find it educational, but not in a condescending or insensitive way. SP C adds that he felt comfortable interrupting PH B when he had questions or required clarification, and that he feels he had the opportunity to ask questions. SP C suggests that PH B's answers were, “very understandable. He kept it nice and plain and simplified as best he could," so patient understanding was optimized during this encounter. Of the proposed procedures, SP C says, "I really had no idea exactly what they were. I only heard the names 'angiograms,' 'angioplasty,' but the way he explained it, I walked out of there being very educated."

Regarding the images, SP C admits, Well, you know, the images that I saw, well, I didn't really know what they meant. I mean, I could see the images, but I didn't quite know what they really meant, and he $[\mathrm{PH} \mathrm{B}]$ was able to explain to me what 
they meant, and I think he, when he was describing the use of the balloon [angioplasty], and he showed me specifically where the [affected] arteries were and how they were different from the other arteries, they were unhealthy, and the reason they were going to push this balloon through was to push all the plaque out and open that up. On the other hand, he also said, "But we probably have to do that again." So, maybe that was the second-best solution for what was going on. Of course, his primary one was the bypass, and that was the one I was confident in.

Although the images were somewhat meaningless until explained, for SP C, they were helpful in providing information during PH B's explanation of both the findings and possible treatment options. SP C admits that some of his attention was focussed on the images during this encounter:

Aware of the images, and not knowing really; the arterial images that he showed to me and explained to me about the three different channels of [arteries]... and he was able to point those out. But I tend to think that when I was looking at the other images, they were actually the inside; I thought they were the inside of the artery that he was showing. That's what I thought they were.

Supporting PH B's sense of the images, SP C does seem to have been distracted by being unable to understand what he was seeing. Regarding how the images were used during this encounter, SP C says, "Although they weren't necessary to explain, but he did.” For SP C, perhaps the discussion alone, without the images, would have sufficed. Regarding PH B's communication, 
SP C says, "I felt confident, and I understood everything he said. I remember all the explanations for each procedure. I really thought it was really buoying me, and giving me confidence and hope, essentially. And yet, knowing that this is serious, but I still had hope."

When questioned about why he had moved his chair before the beginning of the encounter, SP C suggests, "I just felt as if I wanted to be a little more distant from him. I just thought, I felt like adjusting it because I could still see what I need to see. But, it just felt like a conversational space." SP C adjusted the chair to create what seemed to him to be a more conversational space, prior to meeting PH B. SP C felt no further need to adjust the physical space once the interview had started, so perhaps his initial decision was appropriate. SP C adds, however, "But as it turned out, like, he was kind of, he moved up when he wanted to see- he moved closer. And so, I thought that space that I had created, I thought, no it was good, because I didn't anticipate that he was going to move closer. I just felt more comfortable." PH B moved his chair closer to SP C to facilitate the discussion using hard copies of the images. SP C suggests, "I think I might've moved forward as well," which, in fact, he did. SP C concludes, "He shared it [an image] with me." SP C also concedes that they maintained strong eye contact throughout the interview.

When asked if he felt comfortable during this encounter, SP C answers, "I thought it was a really good 'man' interaction." When asked to elaborate, SP C continues, "It was just two guys. Honestly, I felt, lots of times with certain doctors, and I'm not saying that, there's no preference between female and male doctors for me because I've had lovely conversations with female doctors, and felt the same way. You know, I felt the exact same comfort place. It was just a male thing." SP C suggests that the interaction felt, "very easy."

SP C's final comments on his interview with PH B are: 
Well, first of all, I was delighted with it. I was delighted because I thought this guy just aced it so well with what I needed. And, you know, I've been looking at this for the last few days, and there was some trepidation at home about, 'What am I going to do here?' But, it just was so easy, and because he made it easy by coming in and being a very confident guy too. And open, he was very — a modest fellow at the same time. I felt a modesty and a, you know, like, 'I'm no hero here, but this is what I do.' ...The fact is I never, I didn't know any of this stuff anyway. And, so as a person walking down the street, just, I'd been educated coming and doing this thing that you gave me here (chuckles). And I'm not just blowing your horn for you.

Clearly, SP C found this encounter both relaxed and informative. Patient simulators gain knowledge regarding the medical situations they portray, from the role, but also from information provided to them by physicians and medical students. In this case, the encounter was perceived as very educational.

\section{$\underline{\mathrm{PHC}}$}

PH C is a General Practitioner in the GTA with twenty-three years of professional practise at the time of this research. When asked for a general assessment of his encounters, he says, "I think it went well." When asked to expand on this response, he explains that the answers were available in the results, and he was able to explain the answers to these "typical patients" in a way that was reassuring. He also says that he said and did what he normally would in practise. He tried to use "non-technical terms" to ensure clear patient understanding, adding, "I think there were times 
that if there was something they didn't understand, it was good that, you know, they came back and told me, and then I was able to, you know, explain a little bit further or maybe use some other terms." When asked how a practitioner knows whether a patient has understood, he says, One of two ways: either when they repeat back to me how they feel. Okay? I have a good sense of whether they, you know, feel the question has been answered, and whether it's been answered appropriately. And I guess the second way is the next question that they ask. If they ask the next question, it's kind of like the next step after this answer, then I know that they probably understood this one, and they are asking the right thing, and that will kind of lead me.

PH C agrees that this approach requires the physician to actively listen to the patient. Interestingly, his response does not include the obvious answer: just ask. PH C also thinks that these patients had adequate opportunities to request clarification, or ask questions. His focus remained on the patients during these interviews, primarily because both SP A and SP C seemed anxious to know their diagnoses and treatment options. PH C also feels that he applied a patientcentred approach, in that he was, "not only answering the question, but also letting them know what the next step is in terms of referral, in terms of what possibly, you know, the specialist might do. So I try to encompass the whole thing, the whole picture for them, yeah." While this response does encompass the whole medical approach, it does not acknowledge the patients' psycho-social context.

Considering body language, PH C feels that the physical distance between himself and the SPs was fine, and required no adjustment. He also declares that his eye contact was good with both patients. In assessing the patients' comfort levels, PH C suggests, 
I think that they got more comfortable as the session went on. I think initially, obviously, you know, they're kind of uptight. They want to know the result. They want to know what's going on. But once they, sort of, got the answer, they were reassured that it's not heading towards cancer [the Lee Berkowitz role] then I think they felt much more comfortable.

PH C was conscious of a shift in the SPs' demeanours through the course of the interview. When asked if he has any other comments, PH C laughingly states: "I thought it was enjoyable." He then adds, "If the chart said this showed cancer, or bronchogenic cancer or something like that, then that would have changed the whole mood in the encounter and everything." Because he felt that both cases were resolvable and had positive prognoses, he found the experience enjoyable; he felt that he did not have to deliver bad news.

It's always nice to tell a patient that, you know, at least from one specialist's point of view they don't think it's cancer. And, I guess in the second case, that there's no profusion defect, even though there's a ninety percent diagnosis, which is quite a bit, but at least right now there's no profusion defect and we can, you know, sort of move on to the next step.

This declaration by PH C makes it clear that when a physician has to deliver bad news to a patient, it is difficult and can have a personal cost; however, it also makes clear the difference between physician and patient perceptions regarding what might be considered bad news. The Chris Anderson role that PH C encountered has serious cardiac findings that will likely require surgical intervention, a lengthy recovery, and life-style changes for the patient. 
PH C encountered SP A as Lee Berkowitz and SP C as Chris Anderson. When asked for his overall impression of the interview, SP A comments, "Felt very comfortable, and, you know, it was a lot of information, but I felt he was very pleasant, and certainly very personable and he made me feel very comfortable. Yeah. I liked that he showed me actual physical photographs; I like the tactile part of the pictures." When asked if his comfort level changed through the course of the interview, SP A says, "Yes, because he made me feel more at ease. Obviously when he brought up the fact that it might not be totally cured yet, that red-flagged me a lot, especially as a hypochondriac kind of character. So I clung onto that a bit, but having said that, I think he eased it as well." Commenting on PH C's use of language, SP A says he felt the language used was appropriate for the patient's understanding; "it didn't seem overly technical, and if it was, he explained it, which was good. And I like that as a person, because I feel educated." When asked if the encounter felt conversational, SP A responds, "I would say that it was more of a counsel, one-sided thing, but certainly not in a bad way; he told me his story, and he was open to comments." SP A also suggests that this approach was probably wise for Lee Berkowitz-an overly anxious hypochondriac, who, given the opportunity would "derail the train." In terms of opportunities to ask questions, SP A responds, "I certainly felt like I was allowed talk whenever I wanted to. I mean, I would have to sort of butt in a bit to do that, but even so, he was open to it." PH C's answers to SP A's questions were consistently understandable. SP A also observes that towards the end of the interview, when Lee again referred to his fear of cancer, "he [PH C] answered that by saying the radiologist was pretty sure it wasn't cancer, so that was very reassuring." The physician succeeded in creating neither false hope, nor false anxiety in the patient; he was realistic. 
Regarding body language, SP A acknowledges that initially, his focus was on the images on the monitor, adding that he misinterpreted what he was seeing. "The thing I was looking at wasn't even the cancer. It was on maybe the heart probably, or something. And then he pointed out the cancer, or the growth — it wasn't a cancer, so. And I was a bit, I was focused on my neurosis, the hypochondria. And then he relieved it, so then I was more focused on the information he was giving me." SP A thinks that the physician was focussed on him throughout this interview. He also feels that PH C responded well to the patient's sense of urgency for quick, but reasonable, action. Additionally, SP A felt comfortable with the physical distance between himself and the physician, adding,

I thought his body language was lovely. I mean, he was very exuberant with his hands. You know, eye contact continually and, you know, showing me the pictures. It wasn't like, 'I have the cards, and I'm going to tell you what's happening." It was like, you know, it was a conversation that way, for sure.

SP A found it effective when PH C leaned in to show him the images, "and it wasn't condescending or patronizing at all.” In terms of comfort, "I didn't feel uncomfortable, and, if anything, he made me feel more comfortable right off the top." When asked to expand on this comment, SP A says, “...his sitting down, his presence, his eye contact, his, you know, his demeanour, his tone of voice, you know. Yeah, all those things." Clearly, PH C is capable of putting patients at ease, even during a difficult interview.

SP C's opening comment regarding his interview with PH C, is, "Well, the doctor came in, he was extremely friendly, very graceful, gracious, and I had no problem standing up and meeting him, and as soon as I saw him come in, I felt that my day got better just because of his 
manners." When asked if that sense changed through the course of the interview, SP C declares, "I thought he was consistent throughout, and he was more than willing to explain. Every question that I asked, he would pause and let me ask him a question, and he would give me his best answer, so I appreciated being listened to.” Regarding the language used, SP C comments that whenever PH C used words he did not understand, he asked for, and was given, clarification. "Like the narrowing of the arteries. So, there's a name for that, stenosis, or something. He explained what that was." This gave SP C the sense that "he [PH C] was open." Although SP C acknowledges that PH C, "did speak considerably more than I did" he feels that this approach worked well in this interview. "I listened and he explained to me certain things, and that's all I wanted to get." Although SP C feels that he had to interrupt the physician to ask questions, these interruptions were readily accommodated; the physician answered according to the patient's agenda, rather than simply acknowledging and deferring questions until later in the discussion. By addressing questions when they seemed urgent to the patient, SP C reports, "we were on the same page, with the same project in mind as far as my health." PH C's answers were always, "very understandable. He seemed to be inclined to want to explain to me what I needed, and seemed to want to accommodate me with those answers. And so, I just felt really, you know, looked after most of the time. All the time, I should say." SP C also states, "My anxiety level went down considerably with each bit of information I got. And that really helped a lot, because it's - the build up to this, like, I was in a state of anxiety coming in and, but with the doctor coming in and his friendliness, and, you know, his accommodation, I just felt very relieved and looked after." PH C's easy manner helped decrease the patient's anxiety. SP C thinks that the physician's focus was on explaining the situation to him; 
He alternated between, kind of good news and bad news, in a sense; but he'd always come back to the good news and say, "Yes, but you have a really healthy heart, save for these arteries that are being clogged, you actually have a healthy heart." So that was a total relief that, gosh, I have, so I guess if you have a strong heart, that means they might be able to do procedures, and you might accommodate it better.

By maintaining and reinforcing his focus on the good news, PH C helped Chris Anderson recognize that, although serious, the situation could be remedied.

Regarding body language, SP C felt that the physical distance between himself and the physician was comfortable. Regarding eye contact, SP C reports: "Full of eye contact; look at me, point to something, look at me. I was never let go.” Although SP C initially says that he felt no discomfort, he qualifies this by saying,

I did sometimes rock between being a little bit scared of the truth, the explanation of what was going on, but then, a sense of relief when I was given the ability, well, when he mentioned, I think, an angioplasty for instance, and what it would do. Right away I felt better. I just think, oh, that seemed like a simple procedure. It wasn't going to take too long. And I thought, this will make me feel better. And he reassured me that, "Well, you're not going to feel that tightness in your chest anymore if you have this angioplasty." And I think, well, that's just wonderful. 
PH C explained the procedure to this patient clearly, as well as offering the positive impact of this procedure on the patient, all while remaining positive about the strong potential for success. In this way, he was able to build a strong therapeutic relationship with SP C, who exited the encounter feeling both relieved and positive about the next steps.

PH C's efforts to calm the patient, while explaining the results and treatment options, had an enormous impact. SP C reports, "I slowed down as well. Because I was anxious at the beginning, I was, kind of, anxious in trying to get words out. But I slowed down as the information he was giving me, and my opportunity to ask questions and not feel funny about it, I think I was able to slow down too and be able to articulate a little bit better." Due to PH C's efforts, SP C was aware of his anxiety subsiding, allowing him to engage more fully in the conversation.

Ultimately, SP C reports that he found this encounter very educational. "I learned a lot of things I had no idea about." He concludes, “...to think I heard all these terms, many, many times and never really bothered to look them up or what they were, or how, you know. What is an angioplasty? I was never really sure what it was, but he explained and how it works, and what happens with it. Wow, that was all really lovely education, I thought." This is similar to SP C's concluding thoughts during his exit interview regarding $\mathrm{PH} \mathrm{B}$, when he also portrayed Chris Anderson.

\section{$\underline{\mathrm{PH} D}$}

PH D is a Family Practitioner in the GTA with thirty-five years of professional practise at the time of this research. He encountered SP B as Dana Jones and SP D as Skyler Hughes. When asked for his overall sense of the interviews, PH D responds, "The second case [Skyler Hughes] 
was very strange if you're looking at family, like really strange, for family medicine." He then discusses the encounters separately:

Well, first one [Dana Jones] would require a lot of discussion I suspect. And I think, I mean, she was challenging. I expected, probably would have to deal with her a little bit more in terms of the smoking because, as I said to her, "You're in hospital. Let's be realistic about this." With that being said, I think she was, at the end, agreeing with me. I think we were on a mutual path. I see some glitches and problems. My guess is the first time she sees someone who's rough to her, she's going to have a bird. That being said, that's why I said to her, "It's out of my hands. Be prepared." But at the same time, don't give up the ship. Just understand there's good news; bad, and we'll try to find someone else for you. So, she would've been a challenging one. The second one [Skyler Hughes], I think she just wanted a friend to stand by her... That's why I actually said, "Let me talk to the person and give you the questions to ask." Because she was, that's what I was sensing, this is a person I don't have to push along, but this is a person who needs information better than I can give it to you. And that's why I offered that to her, and I didn't offer it to the first one.

While it could be argued that PH D adjusted his communication style to these individual patients, a very patient-centred thing to do, it is also apparent in the encounters and his exit interview that 
he is quite judgemental when patients' ideas of their needs differ from his own, showing a lack of patient-centred approach.

When asked whether he said and did what he would normally do in practise, PH D responds, "No. In the second one, to be honest, I would've been much more straightforward and said, 'This is not my field.' But since I couldn't do that, I played the game as best I could.” He continues, "I hate lying to a patient. I hate misleading them. I was in a bad situation. She was looking at something. I had to say something. I gave it my best shot. We never do that in the office." When the researcher reminds him that he did admit during the interview that it was not his field of expertise, he responds, "But I wouldn't have tackled that far. I wouldn't have gone that far." During recruitment, it was made clear to physicians that this study's focus was communication with older patients in the presence of images—not their knowledge base. Recruited physicians were also told that they were welcome to bring medical resources with them, such as a medical PDA. Additionally, the consent form specifically states: “...you are free to withdraw your consent and end your participation at any time without penalty... At any point in the study, you may refuse to answer any particular question or end your participation.” Although it could be argued that PH D's decision to continue an encounter that was clearly beyond his expertise might indicate his commitment to the research, this decision also shows questionable judgement.

Regarding the appropriateness of language use during his encounters, PH D says, "I screwed up once or twice, but they [SPs] brought me back." He states that he "screwed up" this aspect of the encounters three times during the exit interview. Upon reviewing the encounters, and listening to PH D berate himself over his use of medical terminology with the SPs, it becomes apparent that PH D relies on patients to request clarification of technical terminology, 
rather than explaining or defining terms himself. While SPs are trained to prompt a physician when s/he uses medical language, in practise, most patients will not. The possibility exists that some of PH D's patients do not understand what he tells them, potentially leading to issues of non-compliance or uninformed consent.

When discussing technical language, PH D says, "What's a lipoma?” and then continues into a monologue on cancer. A lipoma is a benign fatty mass (lipid), and such things are relatively common in middle-aged and older people, although usually they appear just under the skin, rather than in the heart. The term lipoma does sound similar to lymphoma (type of cancer), and perhaps $\mathrm{PH} \mathrm{D}$ is confusing the two.

When asked whether he thought the patients had adequate opportunities for clarification or to ask questions, PH D responds that in the second case, the patient [Skyler Hughes] had adequate opportunity. Regarding the first case [Dana Jones], PH D says, "In the real world, the first patient is one that I say, I don't know what they're getting out of this. I mean, she didn't want to have [a biopsy]. If she didn't, it's because she chose not to." While SP B asked perhaps more questions than the Dana Jones role required, the patient was clearly non-compliant, due to personal history and fear. Rather than trying to understand why the patient was responding negatively, PH D seems to blame the patient for being uncooperative.

PH D says that he is familiar with patient-centred communication. When asked if he used it during these interviews, PH D says, “Possibly unconsciously; not consciously. I see a person's needs...I've been around for thirty-five years. I don't do things consciously anymore." In any art, one learns theory to forget theory and simply practise; perhaps the same is true in medicine. In PH D's case, however, his encounters did not reflect a strong patient-centred approach. His 
conscious attention to this approach might support more effective interview techniques, especially with new patients.

Regarding communication, PH D comments that his office has a desk; however, for these types of interviews, he feels the space was appropriate. What he appreciates about the Nursing Lab is that, "You could get close enough to the person. You could look at the person. I felt very comfortable in the situation. It's not one I would have in my office, so sometimes you functionally have to make one if there's a desk. Sometimes you go across and sit beside the person and say, 'Listen, let's talk." A desk would be a barrier between himself and his patients, and for some encounters, PH D prefers that barrier. "If you were trying to be aloof, it [the open lab space] would make it next to impossible. Like, if the first one was really angry at you, it would be literally impossible." PH D seems unable to recognize that it was largely his handling of the interview that evoked this patient's irritation. His stated need for a desk between himself and his patients makes one wonder if he routinely irritates patients in a similar manner.

Regarding patient comfort, he comments that in the second interview, "I got her to laugh," which requires a certain level of comfort. Referring to his encounter with SP B as Dana Jones, PH D suggests that the patient was, “annoyed at me, but not angry at me.” Later he comments, "I don't think there was any opposition with the first one; I think it was more of a challenge, that's all." He adds, "I didn't know if she was trying to break role, or if she was getting comfortable, but she almost smiled at one or two occasions." He then turns the question to his own comfort, saying, "I felt comfortable with both of them, very comfortable." He suggests that he uses humor to help alleviate discomfort in an interview. While he thinks this approach was effective in the second interview, he found, "the first one was a lot more difficult." 
In his encounter with Dana Jones, his attempt to equate doctors with plumbers evoked little response.

PH D comments that, "You didn't give me a case where it was devastating; now, if it was metastatic cancer-but what I do try to do is try to focus on the hope. Ultimately, I will never ever say there is no hope. I will play on the hope issue. I try to play on the positive. There is mortality, but on the other hand, overall things are good." While this is laudable, an ethical boundary also exists to not create false hope for the patient. Additionally, it is unclear whether PH D succeeded in creating hope for this patient. In this context, PH D adds, "The same thing with bringing up the first one's daughter. You think there's hope for your daughter, well...” Although PH D's stated intention in mentioning the daughter was to try to evoke a sense of hope for the patient, this was perceived by the researcher as a manipulation.

PH D found the first interview [Dana Jones] a little unfair, commenting:

To be perfectly honest, with the first one, although I think you guys would probably want me to say it's probably [cancer], I refused to. I wouldn't do that with a patient. I want clear definitions. I don't want to lead you down-I don't want to hurt you; you're already vulnerable. I want to be clear; crystal clear about what we're doing as we go along.

While it is heartening to hear PH D exhibit such sensitivity and concern during the exit interview, again, it is unclear whether the patient felt that she received this care. In both cases, PH D suggests that he would require more information, more test results, before informing the patient of a diagnosis. Obviously physicians should not give patients incorrect information, or devastating health news that is unwarranted; however, this must be balanced with patients' 
frustrations of feeling that inadequate information is being offered, and that the generalist is simply sending one for further tests.

Regarding body language, PH D feels that his eye contact was strong with both patients, and he offers no further comments regarding body language.

When asked for his final thoughts, PH D declares, "If I had that situation, the second case [Skyler Hughes], I would've walked right out (laughs). Give it up! I failed. I failed. Totally failed." While acknowledging that he did a poor job on the second interview, his response underscores that PH D exhibited poor judgement by not refusing to proceed with this interview, or requesting that it be omitted from the research.

SP B's overall impression of her encounter with PH D is:

I think it was successful in that he would've gotten me to have agreed to get the biopsy. So it was successful in that way. If you were saying a strong alliance, you were saying a strong alliance being developed, I mean, how can you do that in that amount of time? Given that person's, given Dana's sort of background, I don't think a strong alliance could be formed.

SP B suggests that ultimately, she would have agreed to the biopsy to provide additional diagnostic information. Similar to the researcher's observation, SP B suggests, “There wasn't a lot of empathetic connection happening in terms of losing my father the way I did, or the bloody mess of my arm with the young woman who didn't know what she was doing" [in preparation for imaging]. She counters this by adding, "He [PH D] had a very cheery, a very upbeat manner, which was really good and sort of proactive. But, with a person who doesn't like doctors, it 
might almost seem as if, since I don't like doctors that much, that kind of cheery, gung-ho sort of, might be, could be a turn off at the same time.” Later, she adds,

I felt uncomfortable, because I didn't want to be with a doctor particularly, but, like I said, this kind of—almost like you're in camp. It was maybe a bit too positive, a bit too- under the circumstance, with this particular person, and he said he would try to get the very best, but - the enthusiasm was maybe a bit much, in that he was with someone who was not enthusiastic about any of this...It's funny; if you're someone who's not that comfortable with doctors, and not that comfortable with talking about your health and that, that sort of eye contact and enthusiasm maybe is not as well received with certain people. It can be an infringement on their personal sort of space.

SP B also notes that she felt PH D's enthusiasm increased throughout the interview; as she says, he seems to be thinking, "If I become even more enthusiastic and more, you know, it's going to convince them more.” SP B says, "But I did consent. I thought I had to do something, but I know, in my head, I was thinking 'My husband's never going to let me not do anything."' Additionally, as SP B notes from the case,

In the back of my mind, I kept thinking, my husband wanted me to come here. My husband wants me to do this, and I have to do something because, really, the husband's nagging, has gotten me toand also I'm worried about this condition. And, I would not want to look around for another doctor. That would be really not something that I would want to do. 
In this encounter, PH D's choice of communication style is not effective for this particular patient; however, SP B feels obliged to give consent because of the physician's (and her husband's) badgering. Is this ethical consent? The physician's agenda is the only one being considered, and the patient's agenda is not acknowledged. As SP B states: "I don't feel like we have anything in common at the end of it." No therapeutic alliance was formed. "It's possible that this person would say they were going to do it and that they wouldn't come back." She indicates that if Dana returned, it might be because of continued pestering by her spouse.

In terms of PH D's response to her difficult imaging experience, SP B says, “I didn’t feel that there was any real sort of empathy." PH D tried to normalize her experience by saying, "A lot of people don't like needles," but failed to explore her individual, and unfortunate, episode. Rather than pursuing this prompt into a conversation, PH D's response shut down this opportunity for dialogue; “There wasn't a conversation,” in SP B's words. As observed, SP B suggests, "I think most of the time was spent trying to get consent [for a biopsy]." SP B states: “Well, there was no question of, 'How do you feel about all of this?'...It was more, 'So, are you going to get the biopsy, or are you going to...? You know, the things that were really important to him." PH D's approach evoked a sense in SP B that, "He was driving the agenda—in a very pleasant, and cheery way_ but he was driving the agenda." Ultimately, SP B felt bullied into accepting PH D's agenda, rather than that she was provided adequate information to make her own decision. During the exit interview, the researcher shared PH D's comment that he could have continued the interview for another fifteen minutes; SP B replies, "I couldn't have."

Regarding the language used, SP B feels that it was appropriate, adding, "Most of the time when he used words I didn't understand, I asked him about it." Overall, the interview was “more closed-ended” on PH D's side. SP B adds, however, “I don't know if that could've been 
avoided, because of the role I was playing, Dana, is not going to be overly talkative in the first place." She does feel, though, that she had adequate opportunities to ask questions, and PH D's answers were understandable.

Regarding body language, SP B spontaneously says during this interview: "Sometimes I wished there had been more of a space between us. I would have preferred it if he didn't lean forward quite as much. Intrusive." She acknowledges that, "It was in an effort to get close. I could see that, because he was very positive." She also indicates that sometimes her focus shifted to the image, "because it was easier to look at the image of my lungs [on the monitor] than it was to always be looking at his face. That's why it was nice to look at the screen; to look away, because I didn't want that much eye contact." She changed the physical space by looking away, down, and at the monitor; however, she did not adjust her chair, making it difficult for the researcher to notice her subtle change in focus. SP B suggests that when PH D tried to persuade her to accept his perspective, "He was very in my space, so it was comforting to be able to look at the screen." The SP feels the physician's primary intention during the interview was on gaining consent, and having her agree to return to him as her family doctor. SP B acknowledges that he defined his role in this context well, saying that he would ensure that she saw the "right" people for further diagnostic tests and treatment.

SP B found PH D's insistence that Dana quit smoking to be annoying. "He wasn't going to quit" nagging her about it. As she states though, "It's one thing to talk about maybe quitting, but that this person is actually going to quit? It's very hard." Quitting smoking was important to PH D’s agenda; however, SP B says, “I don’t think Dana is interested in quitting smoking.” Although PH D emphasized positive results, for instance a noticeable reduction in phlegm within the first week, SP B states, "I'm not sure that I bought that." PH D has failed to gain SP B's trust. 
In conclusion, SP B states:

I thought he was having a really good time, that he relished this, that he just loved this. But it wasn't a good time for me. So, it's kind of a weird sort of feeling when someone is almost excited about the prospect of - of course making someone better, but also the findings, and the discovery. And none of it is appealing for me as a patient. So, you've got one person in the room that's really, 'Come on! We're going to...' You know? And, I don't want to know. So, it's hard.

Again, SP B speaks to the incompatibility of PH D's approach to this particular patient. The patient leaves the encounter feeling even more damaged and bullied than when she arrived. How Dana Jones will proceed after this encounter, is uncertain. If a more successful therapeutic alliance had been formed, her future actions might be more certain and more conducive to helping her regain health.

PH D’s second encounter was with SP D as Skyler Hughes. As discussed in Chapter 7, PH D made what was intended as a benign cardiac condition seem catastrophic, while also admitting that he knew nothing about this condition. When asked for her overall impression of the encounter, SP D states:

The encounter for me was very comfortable. He came in and introduced himself, and asked if it was all right for him to call me Skyler, which is great. And everything, you know, his body language and the way he positioned himself, everything, eye contact, just really put me at ease. And then he asked me, started off with a really nice open-ended question like, "Tell me why you went to see your doctor." 
Clearly, PH D's manner put SP D at ease from the outset. Regarding language use, SP D acknowledges that in some cases, she repeated a word, indicating that she was unsure of the meaning; when prompted in this way, PH D offered further explanation. She also suggests that on a few occasions, PH D caught himself and replaced a technical word with a more common one. SP D feels that PH D provided opportunities for her to clarify his language when necessary. When asked whether the encounter seemed closed, or more conversational, SP D states: "It did feel like a conversation; very much so." She also feels that her questions were immediately answered, rather than deferred for later discussion.

Regarding body language, SP D states, "the eye contact was really phenomenal, and I just felt he was right there with me the whole time." She feels that the physician's focus throughout the encounter was, "Definitely right with me." The physical space between them was comfortable, and the SP felt no need to alter this space. She recognizes that when they were viewing images onscreen, both of them pulled their chairs forward. Reflecting what was observed, their time spent viewing images, "was a very short period in the encounter." SP D did use the optional prompt, "Can you show me the pictures? They might help me understand better." During the exit interview, she says, "I didn't know whether he was going to, you know, show me the pictures, so I asked, and at first he showed me the hard-copy pictures, then we went to the screen." When asked whether she felt comfortable during the encounter, she responds, "Very." Summarizing her thoughts regarding her encounter with PH D, SP D states:

I just found the directness and the honesty really reassuring. Like he told me right near the beginning, you know, "I think this might be what's going on, but one thing I can tell you it is cardiac." And he just made that very, sort of, clear. But he said it in a very, kind of, calm 
tone- " "but I want to let you know that this is cardiac." And I really liked that, even though it was sort of scary to hear. And we did talk about that later. And he was very reassuring about, you know, "Yes, it can be serious with your heart, but, you know, I'll have to see someone else, and it probably will involve surgery," he told me. But that they do incredible things with surgery, and he said that a few times during the encounter, and it made it seem sort of more normal by the end, like, that I would have to be going to see someone else, and it probably would eventually, umm... He also was very, I appreciated that he was very honest about the fact that this wasn't his specialty. And I liked hearing that because he said, "We'll hook you up with who you need to see next, and what needs to happen, but this isn't my_-" One thing he said that could have been very alarming and would have alarmed me a lot if he hadn't said this wasn't his specialty, you know, he said, "In my practice in thirty-five years I've never seen anything like this." And, you know, if he had been a specialist or something, and I had heard that, that would have been very alarming. But, because he said, you know, "Of course, I'm not," That wasn't quite as alarming when he said that.

Although SP D acknowledges some challenges in the interview, overall, her encounter with PH D was satisfactory. This is in spite of PH D's lack of knowledge about the condition with which Skyler Hughes presents, and his creating a much more dramatic situation than was intended. Ultimately, SP D reflects, "One thing that was very reassuring and felt really good was near the 
end, he said, 'What concerns me more than these images and what we see here is the effect that it's having on you and why you're having...' So that just made me really feel taken care of, like these pictures weren't the main story." Although the images were referred to, for SP D, they played a very minor part in her encounter with PH D. This statement by PH D reflected a sense of patient care, and a concern for her wellbeing, well beyond the images and the diagnosis. Unlike his encounter with SP B, in this case, PH D successfully formed a therapeutic alliance.

\section{Conclusion}

Again, it must be acknowledged that it may be inadvisable to translate the findings from a small number of physicians to generalized conclusions; however, some information can still be derived. During the exit interviews, it becomes apparent that the specialists seem more aware than the generalists of the possibility of overwhelming patients with an excess of information, including imaging information. Without adequate training, patients may be unable to comprehend their imaging results, leading to an excess of concern or digressions in conversation. Both PHs A and B are conscious of this possibility, and so are selective in their use of images depending on the accessibility of imaging information to a lay person, and the response of the patient to their images. Although images may be useful in helping explain findings, they make up only a minor part of the discussion; images are not given the chance to dominate the conversation. Both physicians seem comfortable conversing with patients in a relaxed and respectful way that can be perceived as patient centred.

The generalists, PHs C and D, focus less on imaging and more on communicative dynamics during their exit interviews. In both cases, they seem to rely on their patients to keep them on track by prompting for further explanation when needed; while simulated patients are trained to do this, most patients will not. The researcher wonders if some patients leave these 
doctors' offices without adequate explanations, simply because they are unable or uncomfortable in requesting more information. PH D seems to blame his (simulated) patients for communicative inadequacies, rather than examining his own role in the dynamic. PH D in particular seems lacking in an ability to co-create a therapeutic alliance, and seems to want to dominate the conversation as the expert in the situation.

Regardless of their approach, physicians clearly want patients to be educated about their health, and will offer educational materials to ensure that patients receive accurate information. Some physicians want their patients to feel empowered about their healthcare decisions; however, sometimes this seems like a bit of a game, and other times, it is still a case of "doctor knows best." Patients often want to be educated about their health, although this may be a personal preference. Patients are not, however, trained to view images, and may misinterpret what they see, causing conversational digressions and longer than necessary discussions. Images are generally helpful in supporting a physician's explanation to a patient regarding both the diagnosis and treatment options. None of the study participants felt that the images dominated their encounters.

Perhaps surprising is the gap between a physician's idea of bad news, and a patient's idea of bad news. When a physician tells a patient that nothing can be done, and that death is imminent, both parties consider it bad news; however, when a patient hears that s/he may require invasive surgery accompanied by profound lifestyle changes, the patient will likely perceive this as bad news, but the physician may not. A physician who communicates well with patients can help them understand that because something can be done to improve his or her condition, then it really is a good news story. 
Should the roles used in this study be used for future research, some revisions should be made. Although a chart with basic and relevant information was provided, some physicians commented that they would have preferred a more in-depth history and/or additional test results prior to the patient encounter.

This chapter concludes the analysis of field work undertaken for this study. The next and final section offers overall conclusions to this research, as well as recommendations for both patients and physicians in the medical context. 


\section{Conclusion with Recommendations: Where are We Going?}

I'm the first to admit that the resolution of a hand feeling the belly doesn't compare with the resolution of a CAT scan scanning the belly, but only my hand can say that it hurts at this spot and not at this spot. Only my hand can say that.

Dr. Abraham Verghese (under-told stories)

This dissertation set out to begin to answer two primary questions:

$>$ How does the tension between the patient-centred approach and biomedical technologies affect patient-physician communication, especially when older patients are concerned?

Is it possible to optimize practitioner use of biomedical technologies while retaining a patient-centred approach to healthcare?

In a brief archeaology of medical communication, the roots of both patient-centred and biomedical models in Ancient Greece are traced towards a radical shift to the biomedical model through the work of Vesalius and others, creating a more objectified patient body through anatomy. With the professionalization of physicians and continuing developments in medical technologies, culminating in the development of imaging technologies, physicians have increasingly relied on objectifying data for diagnostics, consistently moving away from patient reports of their illness experiences, and thereby challenging the physician-patient relationship.

Against this backdrop, the field research began with interviews of older patients regarding their experiences with medical imaging, and communication with physicians and other healthcare workers before, during, and after their imaging experiences. This data was analyzed using a hybrid strategy, based on the work of Arthur Frank and Andrea Doucet, to create a Foucauldian discourse analysis. This analysis considered the interviewed patients' descriptions 
of emotions, physical sensations, communication through various channels, and thoughts and rationalizations, as well as Foucauldian notions of power, all in the medical imaging context. The analysis revealed both positive and negative experiences, as well as some surprises. It also showed the intelligence, resiliency, and observational and critical skills that many older individuals possess, even though they often feel unacknowledged or dismissed in the medical context. The research also suggests that physicians sometimes make assumptions about what patients want to know, rather than having the conversation to discover an individual patient's needs. Imaging technologies, and the ways in which they mediate patient-physician discourse, can support or impede patient understanding, acceptance, and response. Alarmingly, some cases of uninformed consent were also revealed, pointing to a concerning breach in medical ethics in practise.

Based on the interviews of older individuals' imaging and communicative experiences, simulated patient roles were developed with supporting medical images for physician encounters. Each of four physicians (two specialists and two generalists) encountered two patient simulations with images. To varying degrees, all displayed notions of patient-centred communication techniques during their interviews, although one generalist used a more aggressive ("doctor knows best") attitude that might be considered biomedically driven. During these interviews, very little time was spent on the images; all four physicians remained intent on their patients, using the images only briefly for explanation or reference. The images had little impact on the quality of the interviews. Patients tended to (unconsciously) mimic their physician's imaging viewing pattern; if a physician spent most of an encounter focussed on the images, the patient would likely follow suit. Some instances were recorded of patients and physicians engaging with the images when they were alone in the room. 
Following the physician-simulated patient role-plays, the physicians and simulated patients were each individually interviewed for their thoughts on the communicative dynamics during their encounters. From these interviews, it becomes clear that physicians want their patients to be educated about their health; during encounters, physicians sometimes offered educational materials, and were careful to ensure that patients received accurate information regarding their diagnosis before deciding on a treatment option. Some physicians want their patients to feel empowered about their healthcare decisions; however, in some instances this was more about gamesmanship with the physician holding all the cards. While patients often want to be educated about their health, this is a matter of personal preference; physicians should listen for cues or ask. Because patients are not trained to view images, misinterpretation can be a problem; images require professional interpretation. Physicians found the best way to use the images was briefly to support their explanation of the diagnosis and treatment options, and then to put them away to avoid further distraction. Neither the physicians, nor the simulated patients thought that the images dominated their encounters.

A surprising finding was the existence of a gap between physicians' ideas of bad news, and patients' ideas of bad news. Physicians may only consider themselves to be delivering bad news if the outcome is death, while patients may consider the need for intensive treatment, surgery, or profound lifestyle changes as bad news. Physicians can help patients understand that if a condition is manageable, however critical or difficult, this is still relatively good news.

The findings between the older patient interviews, and the physician-simulated patient encounters do not completely harmonize. Many of the problems older patients commented on during phase one research interviews were not reflected in phase two research encounters. Several things might account for this difference: 
Although older simulated patients were used in phase two research, they were still considerably younger than the interviewed patients. This age difference might evoke different handling by a physician. As noted previously, the youngest of the interview participants seemed to encounter the fewest issues in practise.

The simulated patient-physician encounters only captured one moment in a more complex involvement: the follow-up appointment. Interviewed patients also commented on communication before and during their imaging appointments, experiences that were provided to the patient simulators as background, but not part of their experience during the simulation.

Epstein et al. note that behaviour modification can occur when physicians (and patients) know that they are under observation (1519). The physicians in these encounters knew that they were being observed. While they all bought into the role-play, simulation may cause physicians to behave in an ideal manner, which may or may not accurately reflect their normal practise.

The four physicians who agreed to assist with this research all actively participate in medical education and/or research, as well as maintaining their practices. It is difficult to know whether this sample accurately reflects field practise.

Regarding the question of the tension between the patient-centred approach and biomedical technologies affecting older patient-physician communication, while the interviews point to many adverse effects and some positive impacts, the simulated encounters point largely to positive effects. These physicians seemed conscious of the possibility of imaging technology dominating the discourse, and ensured that their focus remained on their patient through patientcentred communication. This observation helps answer the second question; yes, biomedical 
technologies can be optimized while retaining a patient-centred approach. This challenging combination does, however, require a conscious effort on the part of the practicing physician. Physicians must recognize the potential problem presented by technologies in the room, and practice its avoidance. This can be accomplished through awareness in professional practice, as well as medical education to ensure that physicians can successfully manage the two competing complex tasks of image translation (or any technical competency) and patient communication.

The combination of Frank's notion of letting stories breathe, and Doucet's concepts of the Listening Guide and slow scholarship, guided the researcher's multiple encounters with patient narratives and simulated interviews to support a deep and detailed analysis. As both methodologies allow flexibility, the researcher was able to tailor the approach to the needs of the collected research, rather than mapping a prescribed frame onto the data. While the researcher can only suggest one set of interpretations for this data, another researcher, with different life experiences, might perceive the stories differently, and might emphasize or notice different elements than those observed in this dissertation. Even if the same researcher were to approach the same data at a different stage of personal development, from a slightly different experiential context, the results might have been somewhat different. This dissertation offers one possible set of interpretations of the collected data, although myriad other possibilities exist. Regardless, these tools enabled the researcher to explore notions of the complex communicative power dynamics in process within the medical narrative and dialogical clinical contexts.

Foucault's concepts of the medical gaze and complex power-knowledge relations in the clinical context also inform this study. Assumptions are often made about who holds the power and knowledge in the complex discursive site of the clinic: physicians. Regardless of whether Bichat or Vesalius is considered the initiator of modern medicine and of the medical gaze, both 
figures are foundational to the creation of patient objectification, which supports the power dominance of physicians common to contemporary Western medical practise. Patients generally acquiese, allowing themselves to be perceived through a reductive medical gaze as flattened data; however, as shown in some interviews, patients can resist to regain some power in this relationship.

Medical technologies, such as imaging, provide a further challenge and complexity in the clinical context by introducing a third element in this power-knowledge struggle, and creating another bid for dominance over patients, and even over physicians. Patients and technicians work at the whim and requirements demanded by the machinery, and physicians tend to trust results produced by technology more than their patient's illness experiences. While patients struggle to be perceived as subjects co-creating knowledge of their illness, imaging technologies reinforce patients' status as objects, disembodied data, thereby reinforcing the historical dominant biomedical bias in patients' encounters with physicians. A more productive medical gaze is less reductive and oppressive, observing the living patient as a subject. Older patients are sometimes aware of their ability to empower themselves, often sublty, even when they are ill and in challenging situations. Patients become more comfortable with multiple exposures to an imaging technology, regaining some personal power over the equipment. Patients willingly subject themselves to imaging (and other) technologies, when they trust their physician; however, their trust is enhanced with good communication and a strong theraputic alliance to ensure that their desired outcome is matched by their physician's. In this way, patients and physicians can help each other by sharing power to coproduce an understanding of a disease state, before moving forward towards treatment. 
While much of the onus is on physicians to willingly be more open and patient-centred, some responsibility also lies with patients. With the support of communicative technologies, such as the Internet, patients can be more educated and better prepared for medical conversations. While it may be challenging for older patients who were raised to respect authority figures like physicians, patients would benefit themselves, their care, and their healthcare providers, if they were sometimes a little less complacent and complicit, and were instead a little more questioning and talkative to ensure that their care needs are met, that they have complete understanding of proposed treatment options, and to ensure that physicians have all the necessary information with which to make complete and accurate diagnoses. In this way, power and knowledge can be shared to co-produce more satisfactory results, perhaps more efficiently. Physicians who assume that older patients are overly talkative and off-topic, avoiding asking follow-up or open questions to shut down dialogue, risk dangerous and time-consuming misdiagnoses and ethical breaches. Those who feel pressed for time, would be best advised to apply pressure to funding bodies, or to find alternative patient support, rather than dismissing their patients with sometimes inapporpriate, incomplete, or unsatisfactory care. A more productive dialogue between patients and practitioners, would perhaps cause medical technologies to lose some power in this complex dynamic, and be relegated to the position of subservient tools, as was observed in most of the physician-SP encounters.

In clinical practise, it does seem that biomedical technologies, and their requirements for successful imaging, often take precedence over patient comfort and patient-physician communication. Older patients often feel lost in a complex system, especially if their long-term physician retires. Consequently, they need to self-educate and advocate for their own care. Older patients are conscious of healthcare costs, and critical of perceived waste in the system; however, 
they also feel fortunate to live in a country where their tax dollars ensure that their care costs are largely covered. As a society, we should ensure that increasing healthcare costs are appropriately allocated to provide optimal patient care, rather than necessarily in purchasing the most advanced technologies.

Throughout the course of this dissertation research, medical humanities have steadily gained attention in medical education and professional discourse. The call from patients and patient advocacy groups is for a rehumanized healthcare system in which the communicative relationship between patients and physicians is paramount. Dr. Abraham Verghese suggests that, "What we need in medical schools is not to teach empathy, as much as to preserve it—-the process of learning huge volumes of information about disease, of learning a specialized language, can ironically make one lose sight of the patient one came to serve; empathy can be replaced by cynicism" (The Atlantic). Physicians and other healthcare practitioners are slowly shifting towards medical humanities in education (e.g., the Plymouth University Peninsula School of Medicine $\left.{ }^{120}, \mathrm{UK}\right)$, as well as some practice contexts (e.g., elder care). Supporting and adding to this discussion, this dissertation can make the following practical recommendations based on the field research.

For physicians and other healthcare professionals:

Listen actively and acknowledge patient questions or concerns. Even when test results do not corroborate the patient's illness experience, clearly something is wrong.

Use imaging only when it is warranted.

\footnotetext{
${ }^{120}$ Medical humanities are part of their program, creating well-rounded doctors who more closely resemble the Ancient Greek model of the physician: someone fluent in philosophy, poetry, and the arts, as well as medicine. For instance, medical students go to art galleries with professional artists to improve their observational skills (Dr. Alan Bleakely lecture at Creating Space for Arts and Humanities in the Education of Health Professionals conference, Toronto, May 7, 2011).
} 
Echoing the recommendation of Birkelo et al. and others, images should be reviewed at least twice to ensure accurate interpretation. The logical opportunity for this review is during the follow-up appointment, with either a specialist or a generalist.

$>$ Offer patients the opportunity to view their images, and ensure their understanding. If they refuse the first time, repeat the offer at a later date.

Recognize different learning styles; some patients will understand verbally, others visually, others with tactile models.

Offer creative ways to help patients relax against their anxiety or boredom during long imaging sessions (e.g., music or a recorded story).

When using loud imaging (or other) equipment, ensure that the patient can hear instructions.

$>$ Offer covers to patients in chilly imaging suites; older patients may be more susceptible to feeling the cold.

$>$ Offer to have someone stay in the room with the patient for comfort during long imaging sessions (depending on the safety of the specific imaging type).

$>$ Purchase portable imaging options, such as the GE VScan, ${ }^{121}$ so rural patients can receive necessary imaging in their home hospital. This saves transportation costs, and additional patient stress, with lower equipment cost.

Provide patients with reliable information in the form of pamphlets or recommended websites. While the information may be repetitive or obvious to physicians, it may be

\footnotetext{
${ }^{121}$ The GE Vscan ${ }^{\mathrm{TM}}$ hand-held ultrasound device sells for as little \$5600 USD. For an evaluation of both the technology and its promotion, see my chapter, "The Message in Medical Imaging Media: An Analysis of GE Healthcare's Vscan ${ }^{\mathrm{TM}}$ " in Finding McLuhan: The Man/The Mind/the Message (Regina: University of Regina Press, 2015).
} 
frightening or life-altering for patients. Providing information for self-education improves patient understanding, and their ability to provide informed consent.

$>$ Ensuring patient understanding prior to any procedure is an ethical requirement.

In a stretched healthcare context, consider turning to experienced volunteers to help with patient education. While volunteers already support some treatment and post-operative programs, they could also share their experiences of complex or invasive imaging procedures, such as angiograms.

While efficiency is important, small gestures and brief questions only take a moment, and can provide a profound sense of caring. Introductions are essential.

$>$ Ensure that older or less-able patients can readily move onto and off the imaging gantry. Patient G suggests, “...why didn't you just put one of these triangle trapeze things I could grab on?...then I could grab it and pull myself." This saves patients pain and embarrassment, and allows for more efficiently run imaging suites.

Provide accessible opportunities for patient feedback in a way that allows them to feel safe. Patients should not feel that they are putting themselves at risk by lodging justifiable concerns. Patient feedback ensures improved hospital care, and reduces liability around both personnel and physical contexts.

Remember that the patient is a whole person, a subject seeking professional help. For Medical Students:

Ask for help when needed.

Train with real patients, but ensure that these patients are not traumatized or injured in the process. 
Continue practising needle insertion technique on a dummy or someone other than a vulnerable patient until you are competent.

For Patients (older or otherwise):

Write down questions as they arise; it may be difficult to remember them all during a medical appointment. This also helps the physician ensure patient understanding.

Take notes during medical appointments.

Bring an advocate to medical appointments to help ensure understanding, and ensure that all concerns are addressed.

$>$ Verbalize concerns, and keep verbalizing them until satisfactory answers are given.

Avoid signing consent to any procedure until you feel fully informed regarding what will happen, and why.

Our healthcare system and medical education may require some adjustment. Imaging and other technologies are helpful tools, but they cost millions of dollars and may not always represent the best use of limited hospital budgets. Physicians and hospitals should focus on a broader sense of patient care, not just efficiency. The healthcare system should invest in optimizing its human potential, rather than in more technology. We need more doctors who are not only technically competent, but also patient-centered.

\section{Potential Future Research}

If the roles drafted for this research are used again, some revisions should be made. In addition to the chart notes drafted for each patient by the researcher, a more in-depth history with additional test results should be added. It would be advisable to undertake additional research under the 
wing of a medical education or hospital institution to aid physician recruitment. A larger number of physicians needs to be recruited to substantiate the results.

Further study is required to:

> Substantiate Patient E's claim that male and female patients behave differently in the medical context.

Explore the notion that medical technologies can be perceived to have the power to determine human behaviour and action in the medical context, perhaps through the theoretical lenses of Donna Haraway's cyborg or Katherine N. Hayles's posthuman.

$>$ Ascertain whether patient reactions to imaging technologies can be extrapolated into other medical technologies. Such investigation might take a similar form to the current research, but with patients on dialysis, for instance.

Substantiate the researcher's supposition of an acquiesce narrative in palliative care, adding to Frank's list of patient narrative types. 


\begin{abstract}
Afterword
$\mathrm{PhD}$ candidates frequently suffer from stress-induced illnesses, sometimes with serious manifestations. In addition to the car accident mentioned in the Foreword, a year later, I had to go to emergency in the night due to acute shortness of breath. Scary stuff. Although I have never suffered from respiratory problems, I was initially told that I probably had COPD (chronic obstructive pulmonary disease, which is ultimately fatal), and then that I likely had allergyinduced asthma. Again, I was put through myriad tests, with two series of lung X-rays, two pulmonary function tests, ultimately receiving no definitive diagnosis. Meanwhile, I was prescribed four different types of puffers and a pill-form medication. While these medications improved my breathing, they all featured adverse effects as well. Due to my persistence, a year later I finally had the "gold-standard" asthma test: a methacholine challenge. Subsequently, I was informed that I do not, in fact, have asthma. I have allergies, which I have been aware of since childhood. All but one of the medications I received were not indicated for allergies.

Perhaps more relevant to the current research is my experience of what should have been a routine mammogram in January 2015. An anomaly had been detected in my left breast in 2008. At that time, it was imaged with both mammography and ultrasound, and found to be a benign adenopathy, a diagnosis confirmed in 2010. Without thinking about it, this year, I changed imaging clinics; not only does this mean different equipment with slightly different calibration, but also a different interpreting radiologist. Because the new clinic was located in a hospital, they see the worst, and so are even more attuned to pathology. During the mammogram, the technician said, “Oh, there's something in your left breast. Nothing to worry about, but don't be surprised if they call you back for an ultrasound." I told her that I was already aware of the anomaly in my left breast, so I was not worried. The next week, I returned for the ultrasound.
\end{abstract}


Following that, I received a phone call requesting that I return for a biopsy. With no family history of breast or other cancers, and no reason to suspect anything dire, I suggested comparing the current images to the previous images from the other lab, in the hope of avoiding a biopsy. In spite of eHealth Ontario's claims and advertising, medical imaging is not yet digitally archived in a single repository ${ }^{122}$. With some time and effort, I was able to physically retrieve the earlier images and drop them off at the hospital. After reviewing and comparing the old and new images, the hospital still wanted to do a biopsy. This was when I started to feel anxious. Although I was still 96\% certain that there was nothing to worry about, the medical professionals were concerned enough to make this request; naturally, I began to feel a little less certain that everything was okay.

There was no opportunity to ask questions until I was lying, mostly naked and vulnerable, on the examination table. Ultrasound guided the procedure. While the technician was relocating the anomaly, I asked the two questions I had been formulating. "What are the chances that this is nothing to worry about?" She responded, "Oh, well, the radiologist reported it as 'undefined,' so it's nothing that we look at and say, oh, that's a cancer." Okay, so that's good news. "How big is this thing we're talking about? The size of a pea? A marble?" She responded, "Oh, not even the size of a pea. The size of a really small pea." I began to wonder why we were here. She explained that the "mass" was close to the chest wall, so the physician would have to be careful not to catch a nerve or the muscle. The doctor arrived, and explained that they would be doing a more invasive core biopsy, to ensure an adequate diagnostic sample, rather than a needle biopsy, and that three samples were required—from something less than the size of a pea.

\footnotetext{
${ }^{122}$ In conversation, some physicians have speculated that because the Government of Ontario awarded too many contracts for archival digitization, none of the companies wants to share their proprietary software or information. As a result, information does not flow between these independent contractors, even within the same region (in this case, the GTA) or regarding the same patient.
} 
The doctor explained that she would sterilize and freeze the area, then make a tiny incision through which to insert the core biopsy gun. "This is what it sounds like," she said, pulling the trigger. I jumped. She said, "It sounds like an automatic stapler. I'll tell you before I take a sample." She proceeded with her plan slowly, gently, carefully. This is the best one can hope for. When everything was correctly positioned, she said, "Okay, one, two, three" and fired. The mechanism reverberated through my ribcage like a nail gun. I jumped and tensed automatically, eyes shut tight. My reaction surprised her and she waited for me to relax slightly before removing the gun that cradled a small piece of my excised flesh. I think that this is what a tree feels when a dendrologist removes a core sample, except my flesh has nerves and blood.

Satisfied with the first sample, she returned for the next, carefully reinserting the gun's muzzle into the three-millimetre incision. I felt the tool move and tug inside my breast, against the freezing. Once everything was lined up, she said, "Okay, one, two, three" and fired again. Again, the nail gun inside my chest; instant stabbing pain in my left pectoral muscle, writhing on the table, mouth open in surprise and shock and nausea. Eyes squeezed shut and the pain did not stop. Carefully, she removed the precious sample. I said, "I have to put my arm down." Not waiting for permission, I follow this announcement with this action. “Okay, just don't touch anything," she instructed. I was writhing in pain that refused to stop, while she checked with the ultrasound wand. I imagined leaving, getting up and walking out. Then I imagined returning if the sample proved inadequate. It was better to stick with it. But it hurt. "I'd be really glad about now if you could tell me that you don't need the third sample," I said. "That's what I'm checking for," she said. With the technician's help, they took one last picture as evidence that they had a through-and-through of the "mass." "We've got everything we need" she announced. "You can 
go now." I found myself apologizing for not being a fully compliant patient. She turned from the door, said, "I should be apologizing to you," and left.

It was only then that the technician gave me the after-care instruction sheet, and I realized the extent to which I had been intentionally injured. Apply ice to reduce swelling. Take Tylenol (not Advil or Aspirin which might induce further bleeding). Keep site clean and dry for at least 24 hours. Keep dressing in place for at least three days. Be on guard for signs of infection. Expect bruising for up to three weeks. Avoid heavy lifting for at least 24 hours. This is the number for emergency follow-up. Ensure that you have an appointment for regular follow-up in 10 days. I left in a mild state of shock. My left pectoral muscle was screaming. For at least five days, my left arm and hand were weak, with reduced sensation and movement. Bruising and swelling were apparent for weeks.

The follow-up doctor was someone I had never met. To her credit, she began with, "You're fine. Everything's okay." She later acknowledged, "You weren't worried, were you, but we made you anxious, didn't we?" That's right. I asked, "I wonder if I had to go through this simply to indulge a radiologist's curiosity?" She responded, "Probably, yeah." The ultimate determination? Benign adenopathy.

While I understand that there are potential advantages to mammograms and early cancer detection, this experience was not about early detection. Some people have suggested to me that this was to improve biopsy numbers, which in turn ensure continued funding. While I cannot attest to that, I do know that this was not optimal patient care. I was summoned to the clinic five times, plus visiting another clinic to retrieve records, and underwent an unnecessary and excruciatingly painful biopsy, for no legitimate reason. The finding of benign adenopathy had already been given, and confirmed. The lesson is that different equipment and a different 
radiologist's interpretation may lead to a different course of action, one that, in this case, proved more invasive and problematic for the patient.

The combination of the car accident, breathing issues, and mammogram, have required me to have many more patient encounters with doctors than I have had previously, leading me to reflect on the Western practice of medicine more critically and personally. In my experience, physicians generally listen to my narrative, send me for tests, perhaps send me to a specialist, and prescribe medication. Their attitudes are sometimes condescending (e.g., "If you're diagnosed with asthma, you have to accept it"), and sometimes my own research has led to findings about adverse effects of commonly prescribed medications about which the physicians were unaware. Physicians are just people.

I conclude with the impression that, as a profession, Western medicine has lost its way. Patients seem to be expected to accept illness, and accept medication that may only act as a band-aid solution to reduce symptoms, but not address the underlying problem. Since Bichat, Western physicians have been trained to seek pathology, and many assume that bodies require intervention to heal; meanwhile, patients seek wellness, and may assume that their bodies will naturally heal. I want to feel well, to feel healthy—not medicated. If my body is reacting in a certain way, it is doing so in an effort to communicate, to make me aware of a problem. I want to understand and repair the underlying problem, to support my body's quest for wellness, while medications simply make my body shout a little louder, work a little harder to communicate. Increasingly, I have turned to "alternative" practitioners; my osteopath, massage therapist, acupuncturist, and naturopath. These practitioners respect the wisdom of the body, and believe in the body's innate ability to heal, with patience and the right support. Some health conditions, a broken bone or a bursting appendix, would undoubtedly be more appropriately handled by a 
physician; however, for chronic or long-term conditions, of the type that many older adults experience, alternative healers are more likely to take the time to work with the patient towards optimal health. Bodies require time to heal, and Western medical practice is often impatient. Personally, I believe in whatever works, and often this is a combination of Western and alternative care.

From a Foucauldian perspective, Western medicine seeks to place power firmly in the hands of physicians and other medical personnel, while alternative practitioners tend to act as facilitators, co-creating health with patients, who retain greater control. As our population ages, staying healthy as long as possible becomes even more desirable. Patients are better educated and more able to drive their own healthcare agendas; patients will seek the help they need, whether through traditional or alternative approaches. Helping retain wellness by listening and investing in patient-centred care seems the best investment of time and resources for everyone concerned. 


\section{Appendix A: Recruitment E-Mails}

\section{Recruitment E-mail for Older Individuals:}

Request for Interview

Dear-

I am carrying out a study as part of my PhD research at Ryerson-York Universities. I am contacting you because I would like to interview individuals about their experiences with doctors. The purpose of this study is to explore communication between older patients and physicians when a high-tech medical image, such as an angiogram, CT or MRI scan is also present.

I'm looking for individuals who are 65-86 years old, willing to discuss an experience they've had with medical imaging for a diagnosis a year or more ago. The focus of the interview questions will be your personal experiences with medical imaging technologies (CT, MRI, etc), and your satisfaction with your doctor's communication around the use of these technologies. Some of the things you say in this interview may be included in the dissertation itself, or in the second phase of research to develop roles for simulated patients to portray. Participants will be asked to do an interview of no more than two hours. To compensate you for your time, you will receive $\$ 25$.

Interviews will take place in a private and comfortable setting of your choosing, such as your home, or at Ryerson University. Interviews will be scheduled at a time that is convenient for you. I will conduct all interviews, and they will be audio recorded to ensure accuracy. These recordings will immediately be assigned a number to ensure the security and anonymity of your information throughout the research process. The details of this study have been reviewed by, and receive clearance from, the Research Ethics Board at Ryerson University.

If you are interested in participating, or have question about participating, please contact me at: catherine.jenkins@ ryerson.ca or phone 416-736-2100 extension 77419 (Research Supervisor Steve Bailey). This is a voluntary study and the decision about whether or not to participate is entirely your own.

Thank you for your time and attention,

Catherine Jenkins, PhD Candidate 


\section{Recruitment E-mail for Physicians:}

requesting an hour of your time for $\mathrm{PhD}$ imaging research

Dear Dr-

I am seeking physicians to participate in a research study I am undertaking for my $\mathrm{PhD}$ in Communication and Culture at Ryerson-York Universities. The purpose of this study is to explore communication between physicians and older patients, in the presence of high-tech medical images.

I'm looking for oncologists and cardiologists, as well as family and general practitioners, whose current practices routinely incorporate imaging technologies for diagnostic assessment (e.g., angiogram, MRI, CT, etc). Participants will be asked to review one or two case studies and relevant images, before encountering a simulated patient, whose role is partially based on interviews with geriatric patients. These encounters will be observed by the researcher, as well as video recorded for analysis. A debrief interview with the researcher immediately following the encounter(s) will be audio recorded. Some of the things you say or do in this study may be included in the final research. All recordings will immediately be assigned numbers to ensure the security and anonymity of your information throughout the research process. The details of this study have been reviewed by, and receive clearance from, the Research Ethics Board at Ryerson University.

The case review, simulated patient encounter(s), and debrief interview are expected to take one to two hours, and will take place at Ryerson's Nursing Lab at 415 Yonge Street, Toronto, on one of December 4, 12, and 13, 2012, depending on your availability. Any costs associated with parking or transportation within greater Toronto to participate in this study will be reimbursed. As a token of appreciation for your contribution to this study, you will receive a \$25 Starbucks gift card.

If you are interested in participating, or have questions about participating, please contact me at: catherine.jenkins@ryerson.ca or phone or phone 416-736-2100 extension 77419 (Research Supervisor Steve Bailey). This is a voluntary study and the decision about whether or not to participate is entirely your own. Please feel free to forward this recruitment call to other physicians you think might be interested in participating.

Thank you for your time and attention, Catherine Jenkins, PhD Candidate 


\section{Recruitment E-mail for Simulated Patients}

Dear-

I am seeking simulated patients to participate in a research study I am undertaking for my $\mathrm{PhD}$ in Communication and Culture at Ryerson-York Universities. Please note that this is an independent study, not affiliated with the SP program at the University of Toronto. The purpose of this study is to explore communication between physicians and older patients, in the presence of high-tech medical images.

I will train simulated patients to portray two cases developed for this study from interviews with older individuals, which also require a medical image (e.g., angiogram, CT, MRI). Group training will take place at the Ryerson Library, 350 Victoria St, at 9 a.m. this Thursday January 10, and is not expected to take more than two hours. An alternative training date may be booked if required. The roles being developed include the areas of oncology and cardiology, with both "bad news" and benign findings; as two of these are essentially "breaking bad news roles," you will not be asked to portray a case with personal resonance. You will have approximately three encounters with physicians who will disclose diagnostic results and discuss treatment options. Encounters with physicians will be observed by the researcher, as well as video recorded for analysis. A debrief interview with the researcher shortly after the encounter will be audio recorded. Some of the things you say or do in this study may be included in the final research. All recordings will immediately be assigned numbers to ensure the security and anonymity of your information throughout the research process. The details of this study have been reviewed by, and received clearance from, the Research Ethics Board at Ryerson University.

The encounters and debrief interviews are expected to take two hours each (work days will depend on scheduling of available physicians), and will take place at Ryerson's Nursing Lab at 415 Yonge St on February 20 and 21, 2013. Any costs associated with parking or transportation within Toronto to participate in this study will be reimbursed. In recognition of your professional experience in patient simulation and advanced feedback, you will be compensated $\$ 25$ per hour for training, encounters, and debrief sessions.

If you are interested in participating, or have question about participating, please contact me at: catherine.jenkins@ryerson.ca or phone or phone 416-736-2100 extension 77419 (Research Supervisor Steve Bailey). This is a voluntary study and the decision about whether or not to participate is entirely your own.

Thank you for your time and attention, Catherine Jenkins, PhD Candidate 


\section{Appendix B: Consent Forms}

\section{Consent Form for Older Individuals:}

Title

Patient-Physician Communication: An Examination of the Tensions of the Patient-Centred Model within a Biotechnological Context

Principal Investigator Catherine Jenkins, $\mathrm{PhD}$ Candidate

Co-Investigators Steve Bailey, $\mathrm{PhD}$, Graduate Program Director, Communication \& Culture, York University (supervisor)

You have volunteered to participate in a research study. Before you give your consent to participate, it is important that you read and understand the following information. Please ask as many questions as necessary to ensure that you understand what we will be discussing before signing this consent form.

Purpose of the Study: The purpose of this study is to explore communication between older patients and physicians when a high-tech medical image, such as an angiogram, CT or MRI scan is also present.

Description of the Study: Participants will be asked to do an interview of one to two hours. The focus of the interview questions will be your personal experiences with medical imaging technologies (CT, MRI, etc), and your satisfaction with your doctor's communication around the use of these technologies. Some of the things you say in this interview may be included in a case portrayed by a simulated patient encountering a doctor. Encounters between simulated patients and doctors will be examined to see how the presence of medical imaging technologies affect the conversation, as well as making suggestions about how communication might be improved for future patients.

Interviews will take place in a private and comfortable setting of your choosing, such as your home, the private library at 35 Walmer Road, or at Ryerson University. Interviews will be scheduled at a time that is convenient for you. All interviews will be conducted by the principal investigator and audio recorded to ensure accuracy.

What is experimental in this study? None of the questions used in this study is experimental in nature. The only experimental aspect of this study is the gathering of information for the purpose of analysis, and to help develop cases for simulated patients to portray in the second study phase.

Potential Risks: There are no obvious risks associated with taking part in this study. Interviews will, however, involve some of your time, as well as a discussion of personal medical experiences, which may cause you to reflect on unpleasant memories. If you find the discussion upsetting, you have the right to pause or discontinue the interview. In the event of pronounced emotional or psychological distress, the interviewer may suggest that you consult with your regular doctor. 
Potential Benefits: Unless you find it beneficial to discuss your experiences, you may not directly benefit from participating in this study. By agreeing to participate in this study, you help give voice to older patients and ensure that their needs for understanding are met by doctors in an increasingly technological healthcare environment.

Confidentiality: To ensure your privacy, interviews will be conducted either in your home or in another private area. Interviews will be audio recorded and transcribed for further study, however, your interview will be assigned a number that will be used on all audio, transcript, and computer files. New names will be assigned to cases developed for use by simulated patients in the second phase of this research. Things you say may be used in the development of simulated patient cases; statements you make may be quoted or paraphrased in presentations, publications, or the final dissertation of this research. At no time, however, will this information be attributed to you by name; you will never be named in any publically available documentation regarding this study. Consent forms, or any documentation revealing identifiable personal information, will be stored separately from research data files. All audio and text files will be stored on a password-protected computer and a back-up password-protected USB key. All computer and hard-copy data related to this study will be kept in a secure, locked, private area. This data will only be accessible to members of the research team and a transcriber. The transcriber will be asked to abide by a signed agreement assuring the confidentiality and security of all materials. Interview data will only be used for this study and will be destroyed after five years.

Incentives to Participate: To compensate you for the time, participants will be paid $\$ 25$ for their contribution to this study.

Costs for Participation: No costs are anticipated for participants in this study. Should a participant elect to be interviewed in a location requiring transportation within Toronto, the principal investigator will reimburse this cost if provided with a receipt.

Compensation for Injury: Not applicable for this study.

Voluntary Nature of Participation: Your participation in this study is voluntary. Your choice about whether or not to participate will not influence your future relations with the investigators or Ryerson University. If you do decide to participate, you are free to withdraw your consent and end your participation at any time without penalty. You will not be asked to explain your reasons for withdrawal. At any point in the study, you may refuse to answer any particular question or end your participation. In this event, any information you have already shared will be destroyed immediately and not entered into the study data.

Questions about the Study: If you have any questions about this research, please ask them now. If you have questions about the research later, please contact: Catherine Jenkins at catherine.jenkins@ryerson.ca or phone 416-736-2100 extension 77419 (Research Supervisor Steve Bailey).

If you have questions regarding your rights as a human subject and participant in this study, please contact the Ryerson University Research Ethics Board for information:

Research Ethics Board 
c/o Office of the Vice President, Research and Innovation

Ryerson University

350 Victoria Street

Toronto, ON M5B 2K3

416-979-5042

Agreement: Your signature below indicates that you have read the information in this agreement and have had a chance to ask any questions you have about the study. Your signature also indicates that you agree to be in the study and have been told that you can withdraw your consent to participate at any time. You have been given a copy of this agreement. You have been told that by signing this consent agreement, you are not giving up any of your legal rights.

Participant's Name (please print)

Participant's Signature

Date

Would you like a copy of the final report?
Investigator's Signature

Date 


\section{Consent Form for Physicians:}

Title Patient-Physician Communication: An Examination of the Tensions of the Patient-Centred Model within a Biotechnological Context

Principal Investigator Catherine Jenkins, $\mathrm{PhD}$ Candidate

Co-Investigators Steve Bailey, $\mathrm{PhD}$, Graduate Program Director, Communication \& Culture, York University (supervisor)

You have volunteered to participate in a research study. Before you give your consent to participate, it is important that you read and understand the following information. Please ask as many questions as necessary to ensure that you understand what will be asked of you during this study before signing this consent form.

Purpose of the Study: The purpose of this study is to explore communication between physicians and older patients in the presence of high-tech medical images, such as a CT or MRI scan. You will be encountering simulated patients, whose cases are based in part on interviews with older individuals.

Description of the Study: Physicians will be provided with a brief description and images of the case you are about to see, and given adequate time for review. You will then have an encounter with a simulated patient in which you will be asked to disclose diagnostic results, discuss treatment options, and proceed as you normally would in practice. Cases will reflect the practice area of the physician. These encounters will be observed by the principal investigator and video recorded for further analysis. At the conclusion of the encounter, physicians will be debriefed by the principal investigator; post-encounter interviews will be audio recorded for further analysis. The focus of the debrief questions will be your communicative strategies during the encounter. The case review, simulated patient encounter, and debrief interview are expected to take no more than two hours. Simulated patient encounters and debrief interviews will take place at Ryerson University's Daphne Cockwell School of Nursing practice lab at 415 Yonge Street, Toronto (YNG 338).

What is experimental in this study? None of the simulated patient encounters or questions used in this study is experimental in nature. The only experimental aspect of this study is the gathering of information for the purpose of analysis.

Potential Risks: There are no obvious risks associated with taking part in this study. Encounters and interviews will, however, involve some of your time, as well as participation in a simulated patient care situation that may be stressful, followed by a critical discussion of your professional communication skills. If you find the encounter or debrief discussion upsetting, you have the right to pause or discontinue either activity. In the event of pronounced emotional or psychological distress, the researcher may suggest that you consult with your peer physicians or your regular physician. 
Potential Benefits: Unless you find it beneficial to analyze your practice experiences, you may not directly benefit from participating in this study. By agreeing to participate, you help investigate the experiences of older patients in the increasingly high-tech medical environment, and add to ideas around improved patient care.

Confidentiality: To ensure your privacy, encounters and interviews will be conducted at an educational facility, Ryerson University's Daphne Cockwell School of Nursing practice lab, at a time when it is not being used for classes. Encounters with simulated patients will be observed and video recorded; post-encounter interviews will be audio recorded, however, all data recordings will be assigned a number that will be used on all video, audio, transcript, and computer files. Things you say or do may be quoted, paraphrased, or described in presentations, publications, or the final dissertation of this research. At no time, however, will this information be attributed to you by name; you will never be named in any publically available documentation regarding this study. Consent forms, or any documentation revealing identifiable personal information, will be stored separately from research data files. All video, audio, and text files will be stored on a password-protected computer and a back-up password-protected USB key. All computer and hard-copy data related to this study will be kept in a secure, locked, private area. This data will only be accessible to members of the research team and a transcriber. The transcriber will be asked to abide by a signed agreement assuring the confidentiality and security of all materials. Encounter and interview data will only be used for this study and will be destroyed after five years.

Incentives to Participate: As a token of appreciation for your contribution to this study, you will be given a $\$ 10$ Starbucks gift card.

Costs for Participation: No costs are anticipated for physicians participating in this study. The principal investigator will reimburse costs associated with parking or transportation within Toronto to participate in this study, if provided with a receipt.

Compensation for Injury: Not applicable for this study.

Voluntary Nature of Participation: Your participation in this study is voluntary. Your choice about whether or not to participate will not influence your future relations with the investigators or Ryerson University. If you do decide to participate, you are free to withdraw your consent and end your participation at any time without penalty. You will not be asked to explain your reasons for withdrawal. At any point in the study, you may refuse to answer any particular question or end your participation. In this event, any information you have already shared will be destroyed immediately and not entered into the study data.

Questions about the Study: If you have any questions about this research, please ask them now. If you have questions about the research later, please contact: Catherine Jenkins at at catherine.jenkins@ryerson.ca or phone 416-736-2100 extension 77419 (Research Supervisor Steve Bailey).

If you have questions regarding your rights as a human subject and participant in this study, please contact the Ryerson University Research Ethics Board for information: 
Research Ethics Board

c/o Office of the Vice President, Research and Innovation

Ryerson University

350 Victoria Street

Toronto, ON M5B 2K3

416-979-5042

Agreement: Your signature below indicates that you have read the information in this agreement and have had a chance to ask any questions you have about the study. Your signature also indicates that you agree to be in the study and have been told that you can withdraw your consent to participate at any time. You have been given a copy of this agreement. You have been told that by signing this consent agreement, you are not giving up any of your legal rights.

Physician's Name (please print)

Physician's Signature

Date

Would you like a copy of the final report?
Investigator's Signature

Date 


\section{Consent Form for Simulated Patients:}

Title Patient-Physician Communication: An Examination of the Tensions of the Patient-Centred Model within a Biotechnological Context

Principal Investigator Catherine Jenkins, $\mathrm{PhD}$ Candidate

Co-Investigators Steve Bailey, $\mathrm{PhD}$, Graduate Program Director, Communication \& Culture, York University (supervisor)

You have agreed to participate in a research study. Before you give your consent to participate, it is important that you read and understand the following information. Please ask as many questions as necessary to ensure that you understand what will be asked of you during this study before signing this consent form.

Purpose of the Study: The purpose of this study is to explore communication between physicians and older patients in the presence of high-tech medical images, such as a CT scans. The cases developed for this study are based in part on interviews with older individuals.

Description of the Study: Simulated patients will be trained by the principal investigator to portray two cases developed for this study from interviews with older individuals, which also require medical images (e.g., CT or MRI scans). Group training will take place at a mutually agreed upon time in a private location. As these are essentially "breaking bad news roles," you will not be asked to portray a case with personal resonance. You will have two or three encounters with physicians who will disclose diagnostic results and discuss treatment options. These encounters will be observed by the principal investigator and video recorded for further analysis. At the conclusion of the encounter, and after physicians have been debriefed, simulated patients will also be debrief by the principal investigator; post-encounter interviews will be audio recorded for further analysis. The focus of the debrief questions will be on your reaction to the verbal and non-verbal communication during the encounter. The encounters and debrief interviews are expected to take two hours each (work days will depend on scheduling of available physicians). Encounters and debrief interviews will take place at Ryerson University's Daphne Cockwell School of Nursing practice lab at 415 Yonge Street, Toronto (YNG 338).

What is experimental in this study? None of the encounters or questions used in this study is experimental in nature. The only experimental aspect of this study is the gathering of information for the purpose of analysis.

Potential Risks: There are no obvious risks associated with taking part in this study. Encounters and interviews will, however, involve some of your time, as well as participating in a simulated patient situation that may be stressful, followed by a critical debrief of the encounter. If you find the encounter or debrief discussion upsetting, you have the right to pause or discontinue either activity. In the event of pronounced emotional or psychological distress, the researcher may suggest that you consult your regular physician. 
Potential Benefits: Unless you find it beneficial to analyze your simulated patient experiences, you may not directly benefit from participating in this study. By agreeing to participate, you help investigate the experiences of older patients in the increasingly high-tech medical environment, and add to ideas around improved patient care.

Confidentiality: To ensure your privacy, encounters and interviews will be conducted at an educational facility, Ryerson University's Daphne Cockwell School of Nursing practice lab, at a time when it is not being used for classes. Encounters with physicians will be observed and video recorded; post-encounter interviews will be audio recorded, however, all data recordings will be assigned a number that will be used on all video, audio, transcript, and computer files. Things you say or do may be quoted, paraphrased, or described in presentations, publications, or the final dissertation of this research. At no time, however, will this information be attributed to you by name, although the name given to the role you are portraying may be used; you will never be named in any publically available documentation regarding this study. Consent forms, or any documentation revealing identifiable personal information, will be stored separately from research data files. All video, audio, and text files will be stored on a password-protected computer, and a back-up password-protected USB key. All computer and hard-copy data related to this study will be kept in a secure, locked, private area. This data will only be accessible to members of the research team and a transcriber. The transcriber will be asked to abide by a signed agreement assuring the confidentiality and security of all materials. Encounter and interview data will only be used for this study and will be destroyed after five years.

Incentives to Participate: In recognition of your professional experience in patient simulation and advanced feedback, simulated patients will be compensated \$25 per hour for training, encounters, and debrief sessions.

Costs for Participation: No costs are anticipated for simulated patients participating in this study. The principal investigator will reimburse costs associated with parking or transportation within Toronto to participate in this study, if provided with a receipt.

Compensation for Injury: Not applicable for this study.

Voluntary Nature of Participation: Your participation in this study is voluntary. Your choice about whether or not to participate will not influence your future relations with the investigators or Ryerson University. If you do decide to participate, you are free to withdraw your consent and end your participation at any time without penalty. You will not be asked to explain your reasons for withdrawal. At any point in the study, you may refuse to answer any particular question or end your participation. In this event, any information you have already shared will be destroyed immediately and not entered into the study data.

Questions about the Study: If you have any questions about this research, please ask them now. If you have questions about the research later, please contact: Catherine Jenkins at at catherine.jenkins@ ryerson.ca or phone 416-736-2100 extension 77419 (Research Supervisor Steve Bailey). 
If you have questions regarding your rights as a human subject and participant in this study, please contact the Ryerson University Research Ethics Board for information:

Research Ethics Board

c/o Office of the Vice President, Research and Innovation

Ryerson University

350 Victoria Street

Toronto, ON M5B 2K3

416-979-5042

Agreement: Your signature below indicates that you have read the information in this agreement and have had a chance to ask any questions you have about the study. Your signature also indicates that you agree to be in the study and have been told that you can withdraw your consent to participate at any time. You have been given a copy of this agreement. You have been told that by signing this consent agreement, you are not giving up any of your legal rights.

Simulated Patient's Name (please print)

Simulated Patient's Signature

Date

Would you like a copy of the final report?
Investigator's Signature

Date 


\section{Appendix C: Semi-Structured Interview Questions for Older Individuals}

\section{Introduction:}

Thank you for agreeing to meet with me to discuss your experience with medical imaging. Before we begin, do you have any questions?

\section{Questions:}

1) Please tell me about your encounter with medical imaging equipment (e.g., CT scan, MRI, angiogram, etc.).

2) Was the medical imaging procedure well explained, or were there surprises?

3) How did you feel during the imaging appointment? After the imaging appointment?

4) Were the imaging results well explained by your physician, or did you have questions that remained unanswered? If there were unanswered questions, what prevented you from getting the answers?

5) How did you feel during the follow-up appointment to discuss the imaging results with your doctor?

6) How would you describe your relationship with your doctor? With other professionals you encountered during or after these appointments?

7) Having had this experience with medical imaging, is there anything you'd like to change that might improve such an experience?

8) Can you think of ways that your communication with your doctor might be improved?

\section{Closing:}

Is there anything else you'd like to tell me about your experience? Do you have any other questions?

Thank you. 


\section{Appendix D: Simulated Patient Roles, Physician Instructions, and Images}

\section{Cancer Role (benign):}

Intent: These are physician-driven, conversational encounters. If the physician says something that is not clear to you, you might ask for clarification. If the physician offers to show you the images, you will be interested. You will talk about the imaging experience and/or your fears around your current health situation, only if the physician provides the opportunity.

Patient: Lee Berkowitz, age 66

Opening Line: It's cancer, isn't it?

Prompt (Things the patient might ask): Can I see (the images)?

Note: This prompt should only be given if yours is the second role a physician is seeing and only if s/he has not already offered to show you the images. If the physician refuses, allow the encounter to end naturally.

Medical History: You are a healthy, but anxious person; a bit of a hypochondriac. You are currently asymptomatic; the findings were from a routine chest X-ray during a physical three weeks ago. Dr Herman described a "shadow" on the X-rays, causing him to send you for a CT scan. Dr Herman is now on holiday, but you don't want to wait for his return to get these results, so you insisted on speaking to another doctor.

Imaging Experience: You were relieved to finally get the CT scan, sure that it would provide some answers. You arrived twenty minutes early for your eleven p.m. appointment. You were asked to change into a hospital gown, ensure that any metal objects were removed, and then wait in the hall until called. You felt very exposed, as there were other patients also waiting, and the chair was hard and uncomfortable. You waited for nearly two hours before you were called. The technologist didn't shake hands or introduce himself; he just asked you to follow him. You thought he was rude. The CT imaging suite was cold and the scanner was bigger than you expected (a bit intimidating), but you were here for answers, so you had to overcome your fears. You were asked to lie down on the bed extending from the machine. Once in position, the technician wanted to insert an IV line in your arm for the contrast medium to enhance the images. You'd read that some people are allergic to contrast medium, but after a series of screening questions, the technician decided it was safe to proceed. You happily accepted his offer to use hypoallergenic tape to hold the IV in place. You experienced a flush of heat when the IV started, but it quickly passed. The technician explained that you would need to remain completely still throughout the exam, and that he would ask you to hold your breath for intervals during the procedure. He then asked you to raise your arms above your head, which was somewhat uncomfortable. The bed emitted some whirring and clicking sounds as it moved into the scanner. As soon as you entered the machine, you became claustrophobic and wanted out. 
You felt panicky and twitchy. The technologist reminded you several times to lie still, but you couldn't; there was obvious frustration in his voice. He stopped the scan to talk to you. When he suggested that some music might help, you agreed. He played something soft and soothing, you closed your eyes, and the scanner started again. This time you felt more relaxed and were able to comply with his instructions. Several times, the technician asked you to hold your breath for about 20 seconds. Eventually, you were released from the machine, and asked to wait for confirmation that the images were clear, before you could leave. A few minutes later, you were told that you could go and the IV line was removed. When you left the hospital in search of a taxi, you realized that it was 2:00 a.m.!

\section{Further information to help explain the imaging process to the SP:}

For a short video on the patient experience of the CT procedure, refer to: http://www.youtube.com/watch?v=uHu9aa0QDiE

This video clip offers a physician's explanation of CT scanning: http://www.youtube.com/watch?v=yTZ7cCU4EFA

Medication: While you don't currently take any prescription drugs, your bathroom cabinet is full of over-the-counter medications that you take at the slightest indication. These include antacids, analgesics, antihistamines, cold and flu remedies, laxatives, etc.

Affect: You're a bit of a hypochondriac. You suffer from colds, headaches, gas, etc. and believe that you have arthritis and allergies, although these conditions have never been diagnosed. Your conditions are a big deal to you, although doctors don't seem to take your complaints seriously. You change doctors every few years, and often ask for referrals to specialists, just to get someone to listen to your problems. Although the current findings justify your concern, there is also a sense of vindication ("I knew there was something wrong!") even though you have no symptoms. You're sure that the results of this test will reveal that you have cancer.

You visit your current family doctor, Dr Herman, at the slightest indication; anything from a cough to a new skin spot will prompt you to make an appointment. You often e-mail him with questions or concerns. Additionally, you insist on a complete annual physical, including any additional tests you've read about, even if they're not covered by your health plan. You spend hours online reading about health-related topics; illnesses, new tests, and treatments ("I'm knowledgeable. I read. I go on the Internet."). You believe that it's comforting to know the details about your health. When you read about some new illness, you often think you're suffering from it, because your symptoms seem to fit.

You tend to be very judgemental of others, especially those in the medical professions. You think they should be courteous, listen to you, and answer all your questions. You don't understand why sometimes they seem abrupt, dismissive, frustrated, or rude. If frustrated, you will become quite assertive and demanding in your quest for answers. If a strong therapeutic 
alliance is formed, you may be inclined to open up about your imaging experience and chronic health problems (e.g., the long wait, "I knew there was something wrong," ad lib any breathing or chest problems), given the opportunity. You're happy to share your medical concerns with anyone who'll listen.

Allergies: Although you're convinced of your environmental and food allergies (dust, mold, pet dander, pollens, nuts, dairy, eggs, etc.), and have had a battery of tests over the years, the results have always been either negative or inconclusive. As far as the doctors are concerned, you have no allergies.

Immunizations: Up to date, including flu shots and additional shots (e.g., pneumonia, hepatitis, shingles, etc.)

Personal History: You were an agent with State Farm Insurance for forty years, from age 25 until retiring last year. You are financially secure, with a good pension, and have lived in the same condo for over twenty-five years. Although you dated when you were younger, you never found the right partner, and remained single, with no children. You're still adjusting to retirement and find it hard to fill the time. This means you spend even more time online, pouring over the daily news, and fretting about your health. Sometimes you go to afternoon matinees, or walk in the park near your house, but it's a pretty lonely existence, with few friends or hobbies. You're not depressed, but could be heading there; just remember that this isn't a psychiatry role.

You were the only child of a stay-at-home mother who doted on you. She took you to the doctor frequently, and that ingrained pattern lasted into adulthood. Working in insurance made you very aware of how suddenly accidents or ill-health can happen, and further supported your hypochondria.

Family History: Both of your parents are alive and well; your father, a retired plumber, is 91 and your mother is 89 . Your father's eyesight is failing, and he uses a cane to stabilize himself when walking outside, but he is otherwise healthy. Although your mother is generally well, and has not been diagnosed with anything, she complains about numerous aches and pains. You visit them once a week for dinner, and to help with household chores that they are no longer able to do. You have no siblings, aunts or uncles, and are unaware of any family history of significant disease.

Lifestyle: You don't drink, smoke, or do drugs, and never have. Your diet consists of healthy, high-end prepared foods, augmented by meals your mother sends home with you. You thought that maybe when you retired you'd start cooking, but that hasn't happened. There's a swimming pool in your condo building, and you do many lengths three or sometimes four times a week. 
Instructions to Physician: You are about to meet Lee Berkowitz. After a routine chest X-ray revealed a suspicious mass, Lee had a chest CT last week. His/her FP, Dr Herman, is on vacation and the patient insists on seeing a physician to discuss the results. After reviewing Lee's chart and imaging, discuss your diagnosis and treatment plan with the patient. Answer any questions the patient may have. During this encounter, please do and say what you would normally do and say in practice.

\section{Chart Note: Lee Berkowitz}

S: 66 y/o

asymptomatic; routine Px; well-circumscribed opacity within the LUL on chest radiography no smoke, no alcohol, no street drugs

Meds: No

Allergy: NKA

PMH: None

Fam Hx: mother A\&W; father A\&W

O: T 37 BP 125/82 (NB “white coat syndrome” BP 200/140) HR 70 reg

A: Neoplasm: bronchogenic carcinoma; hamartoma; bronchial adenoma; granular cell myoblastoma; mesenchymal neoplasms; leiomyoma/leiomyosarcoma; fibroma; neurofibroma; lipoma; lymphoma; solitary metastasis; colon carcinoma Infection: septic embolus; staphylococcus; round pneumonia; pneumococcus; legionella; nocardia;

fungi; lung abscess; infectious granuloma; tuberculosis; histoplasmosis; coccidiomycosis; cryptoccoccosis; parasitic; echinococcal cyst; amebic abscess Collagen Vascular Disease: necrobiotic nodule; rheumatoid lung; Wegener's granulomatosis Vascular: infarct; AVM; pulmonary artery aaneurysm; hematoma Airways: congenital foregut malformations; bronchogenic cyst; sequestration; mucocele; infected bulla

Miscellaneous: amyloidoma; round atelectasis

P: CBC; chest X-ray

$\mathrm{CBC}$ and Px within normal limits

f/u CT

\section{Images:}

See two X-rays and three CT scans at:

http://rad.usuhs.edu/medpix/cow_image.html?quiz=\&pt_id=11498\&imageid=39557\&widgets=-1\&start=$1 \&$ mode=\#top

Findings: "PA chest radiograph demonstrate a well circumscribed opacity within the LUL. The lateral view demonstrates a well circumscribed opacity within the LUL, obscuring the trachea. 
Axial contrast enhanced CT demonstrates a well circumscribed nodule in the LUL, with a lobulated border, and multiple clumps of calcium or 'popcorn' calcifications, as well as discrete areas of fat dispersed throughout the lesion." ${ }^{, 123}$ Consistent with Pulmonary Hamartoma.

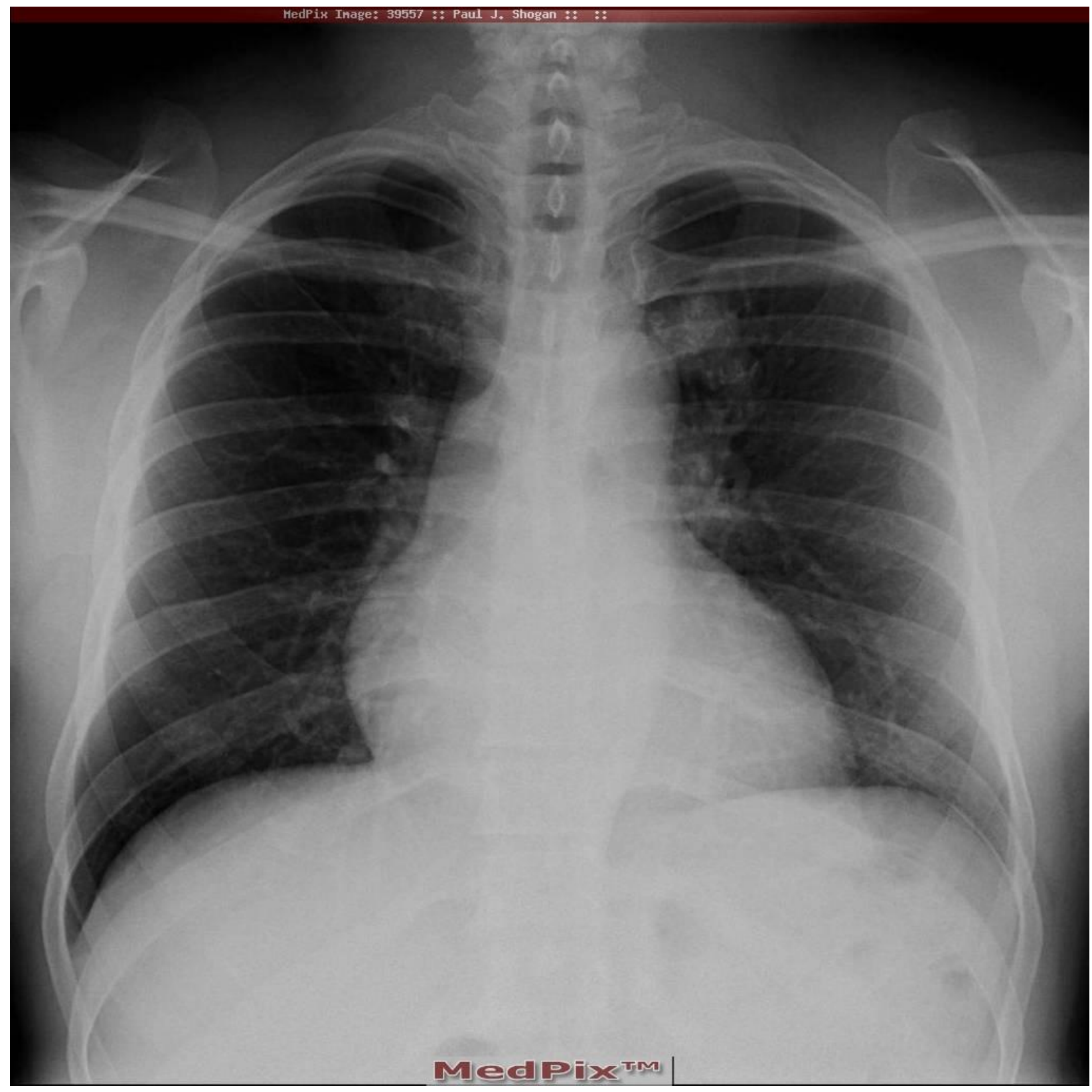

${ }^{123}$ Shogan, Paul. Case 11498 MedPx ${ }^{\text {TM }}$ Medical Image Database, Atlas, and Teaching File, Department of Radiology and Radiological Sciences, Uniformed Services University (Bethesda, MD). Web. 4 January 2013. < http://rad.usuhs.edu/medpix/tf_case.html?mode=case_viewer\&case_prog=cow image.html\&th=-

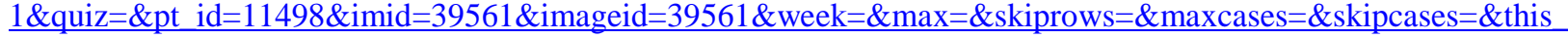
week=\&conf=\#diagnosis $>$ 


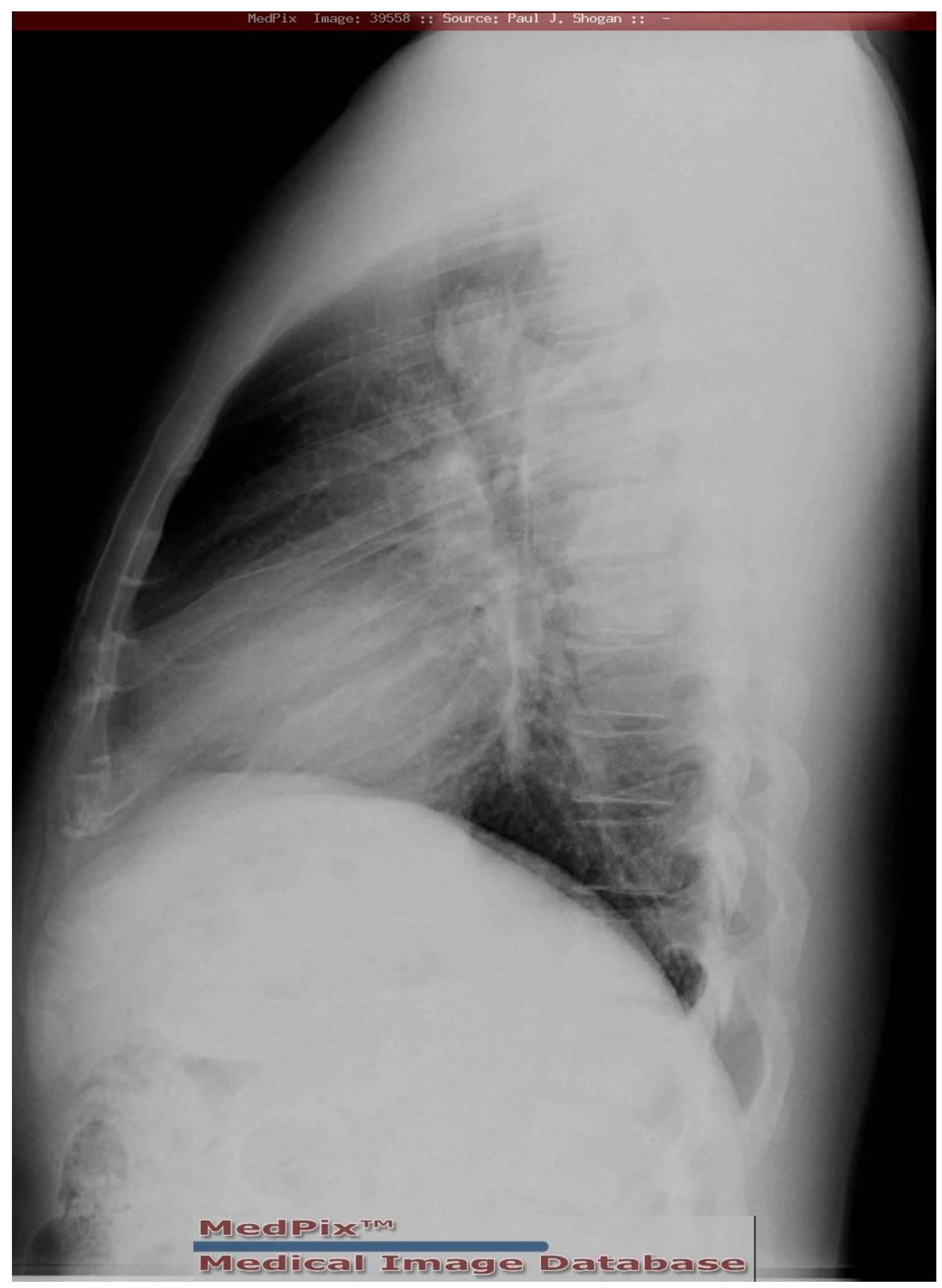




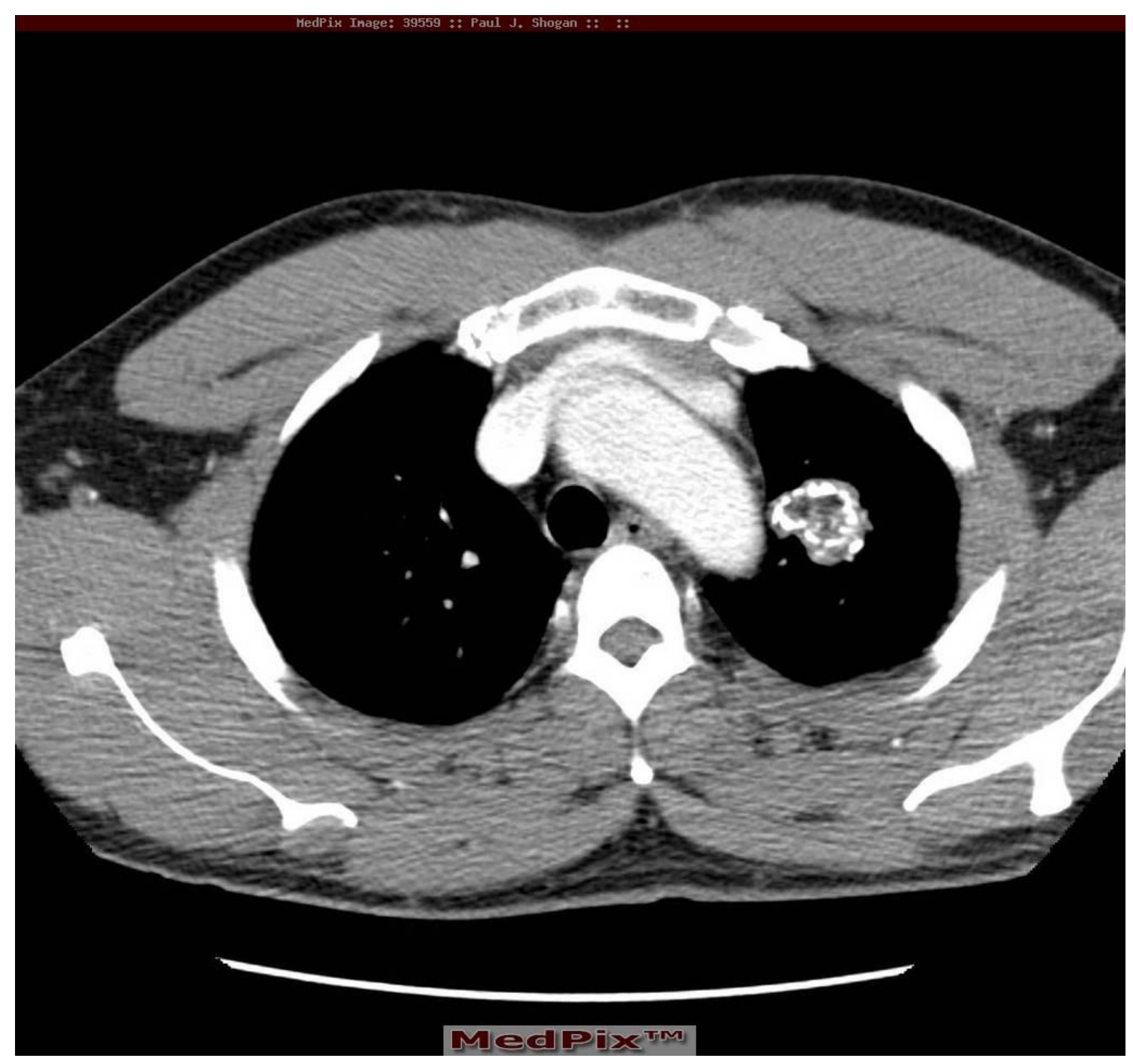




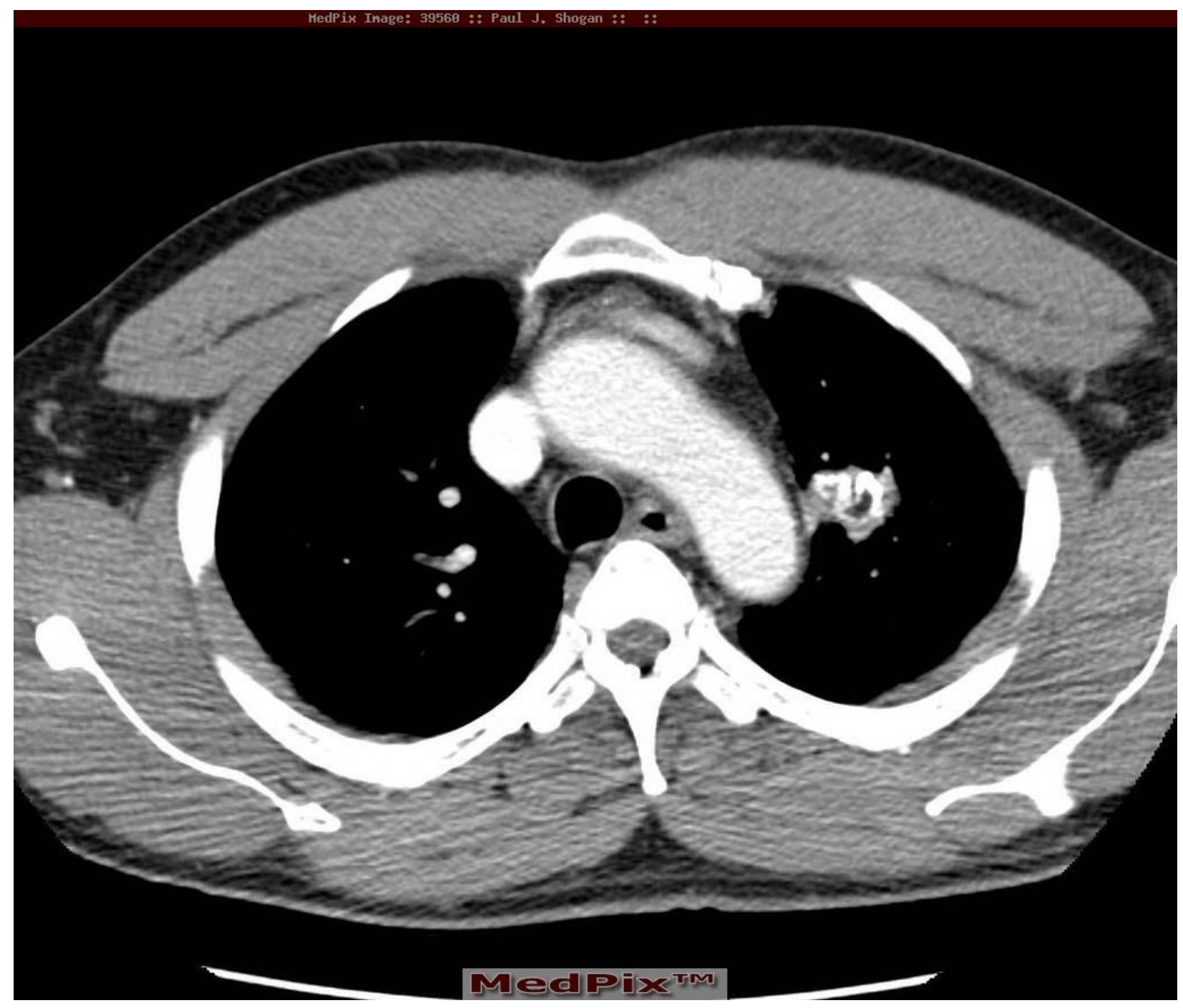




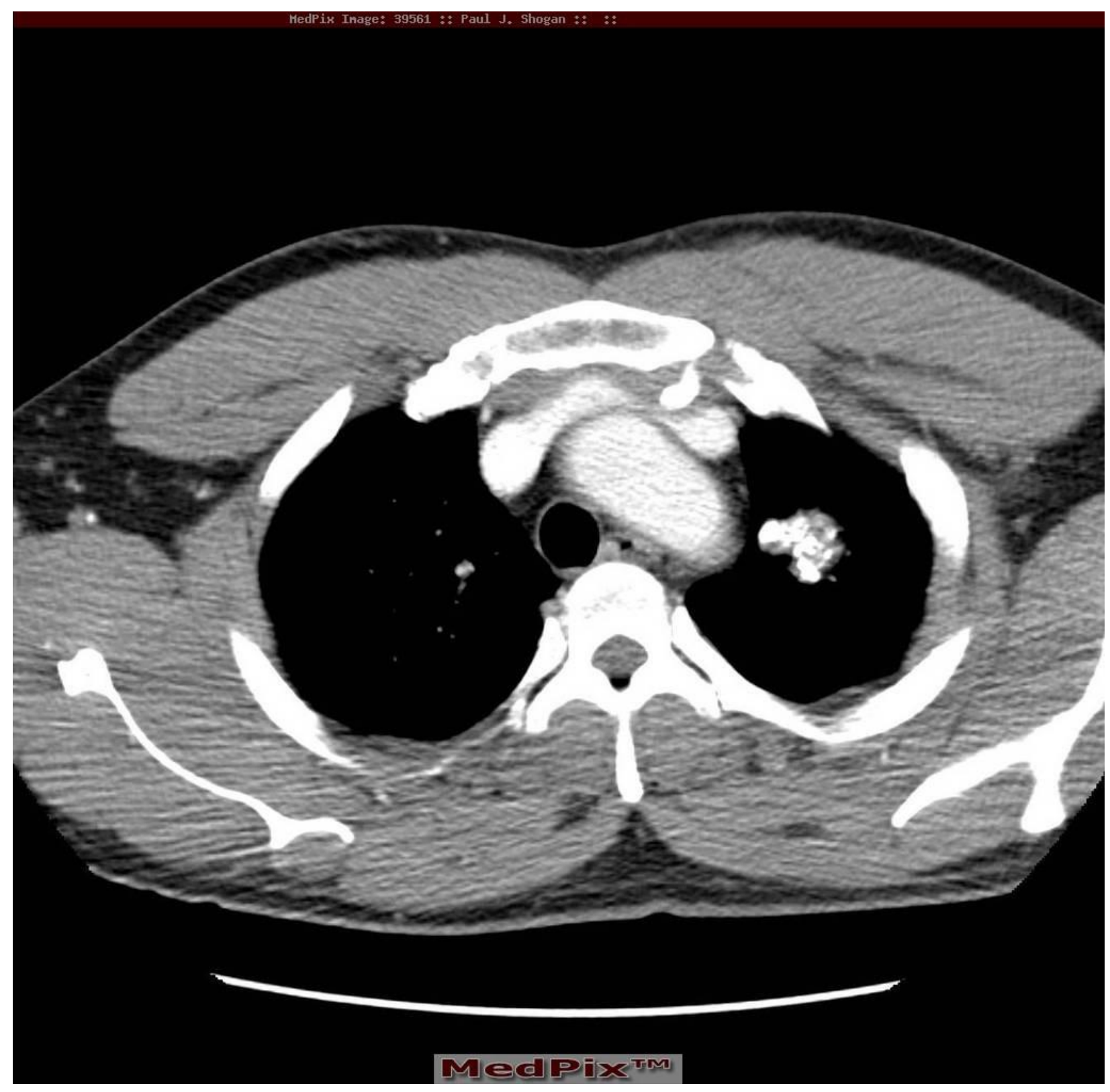




\section{Cancer Role (findings):}

Intent: These are physician-driven, conversational encounters. If the physician says something that is not clear to you, you might ask for clarification. If the physician offers to show you the images, you will be interested. You will talk about the imaging experience and/or your fears around your current health situation, only if the physician provides the opportunity.

Patient: Dana Jones, age 67

Opening Line: Well, what have you found now?

Prompt (Things the patient might ask): Can I see the pictures? It might help me understand.

Note: This prompt should only be given if yours is the second role a physician is seeing and only if s/he has not already offered to show you the images. If the physician refuses, allow the encounter to end naturally.

Medical History: Two weeks ago, you showed up at a walk-in clinic after noticing that your usual morning cough was very phlegmy and brown and you were experiencing increased coughing and shortness of breath. Suspecting bronchitis, or possibly COPD, the clinic doctor, Dr Rontowski, sent you for a chest X-ray, which revealed a suspicious mass in one lung. You were subsequently sent for a PET-CT scan and are here now for those results. You've noticed increased fatigue and some weight loss (maybe 10 pounds) over the last month or so, but didn't mention these symptoms to the walk-in doctor, because you didn't see how they could be relevant. If a physician asks directly about these symptoms, you will be forthcoming. Other than the present illness, you feel fine, except for some occasional knee and back pain.

Imaging Experience: In spite of your smoking history, your increasing symptoms, and your family cancer history, you were surprised that the X-ray showed something in your lungs. Even though you don't like doctors and hospitals ("That's where people go to die"), your spouse kept badgering you to find out what was going on, so you kept the late-night PET-CT scan appointment. In the first waiting room, you filled out a long form regarding previous medical procedures or possible implants, then you were shown into a curtained cubicle and asked to change into a hospital gown and remove anything metallic. You then moved into another waiting area, with other gowned patients; it was embarrassing and uncomfortable to be "sitting in a room full of sick people." A very young woman, a student perhaps, explained that she would need to insert an IV catheter through which a radioactive tracer would be injected to help with the imaging; you don't like needles, but gritted your teeth and submitted to the procedure. She seemed inexperienced, and had a great deal of difficulty finding a vein. Rather than requesting help from her supervisor, she "made a bloody mess of my arm." After several minutes and numerous failed attempts, her supervisor finally intervened, placed the IV, then readily injected the tracer; however, the incident further undermined your confidence in the medical system and, although you would be reluctant to admit it, it was frightening. You were then informed that you 
would have to wait another hour for the tracer to circulate, before the imaging procedure could begin. The long wait in a room with some very ill-looking people further increased your anxiety and got you thinking about mortality. Eventually, you were led into a quiet, cold room where a huge doughnut-shaped machine was waiting. You were asked to get up on the table, so your head would enter the machine first, and stretch your hands above your head, fully exposing the chest. Although this position was uncomfortable to maintain, the scan itself was no big deal. You were given very specific instructions to lie still, as well as when to inhale, exhale, and hold your breath (sometimes for up to 20 seconds). The scan itself took about 30 minutes, but you were at the hospital for over two hours. You were happy to finally leave.

\section{Further information to help explain the imaging process to the SP:}

This short video goes through the PET-CT experience step-by-step from the patient perspective: http://www.youtube.com/watch?v=qCT3KQitrCQ

Medication: You're not currently on any medications. Dr Rontowski gave you a sample inhaler of "Vento-something" (Ventolin; you will recognize the name if it's mentioned), suggesting that it might temporarily relieve your symptoms. You tried it two or three times, but it didn't seem to make any difference, so you stopped using it. You take Tylenol for occasional knee and back pain, maybe twice a month.

Affect: You generally avoid doctors ("All they do is tell me to quit smoking and I'll smoke if I like!"), however, this persistent phlegmy cough and increasing shortness of breath, along with your partner's nagging, have prompted you to seek medical attention. You don't really want to be here, and you feel vulnerable about your current undiagnosed medical condition. Now that the doctors think that there might be something serious wrong, you're anxious to begin treatment, get it over with, and get on with enjoying your retirement. You don't have a regular doctor, and rely on walk-in clinics when needed. Your last check-up was years, maybe even decades, ago.

You have childhood memories of your father's lung cancer and remember being angry that the doctor couldn't save him; this is the root of your general mistrust of the medical profession. "You feel fine until they tell you you've got something wrong with you!" You're also intimidated that doctors are much more educated than you are; you will probably speak very little during the encounter, for fear of sounding stupid. Because of their advanced education, you also have unrealistically high expectations of what doctors can do. You expect that they'll "just give me a pill to fix me up," and don't understand the relevance of lifestyle choices on your health. You don't plan to quit smoking, and a physician would have to make a strong and clear argument, perhaps by showing you the images, for you to consider a behaviour change.

If frustrated or frightened, you might shut down, completely stop talking, and avoid eye contact. If a strong therapeutic alliance is formed, you may feel calmer and be more inclined to open up about your imaging experience and fears (e.g., mortality issues, family history), given the 
opportunity. Please wear long sleeves to hide IV punctures from the difficult needle insertion during the imaging procedure.

Allergies: No known allergies, although sometimes the coughing has made you wonder if you've developed environmental allergies.

Immunizations: You know you had shots to travel to Canada when you were a child, but you don't remember when your last shots were.

Personal History: After high school, you worked on the factory floor at the Dupont plant in Mississauga until retiring two years ago. You have a good pension and are comfortable financially. You married a co-worker when you were both in your early twenties. In spite of occasional marital stresses, you've stuck together and managed to pay off the mortgage and raise three children. You've lived in the same neighbourhood for decades and it's a close-knit community. Since retiring, you enjoy spending more social time with friends at the pub and helping with community projects. Your partner retired the same year and you've enjoyed travelling to Florida for a few weeks the last two winters. Although you never got past high school, you were determined to do better for your own children. Your eldest daughter quit university to marry and now has two children; the second-oldest, a son, is a lawyer; and the third, another son, is finishing a graduate degree. You're very proud of their accomplishments.

Family History: Your father was a Welsh coal miner and heavy smoker, who died from lung cancer in his mid-fifties. Your mother held the family together and brought you to Canada in the mid-1950s, when you were ten. Your mother died in 2000, at age 90, from heart failure. You are the youngest of five children: the eldest, a heavy smoker, died from lung cancer seven years ago at age 70; the second-oldest died from a heart attack three years ago at age 72. The other two live in the GTA and you see or talk to them three or four times a year; they are currently in good health at ages 69 and 73 .

Lifestyle: For fifty years, you have smoked a pack to a pack-and-a-half of cigarettes per day. You enjoy beer, drinking one or two glasses most days, sometimes more, at the pub. You have never done street drugs. Your diet now consists mostly of prepared foods; with the kids gone, you and your partner don't feel the need to set a good example and frozen dinners are quick and easy. In the last few weeks, your partner has commented that you're wasting food by not clearing your plate; this is unlike you, but you don't feel very hungry. Your work was frequently very physically demanding, however, since retiring, your exercise has decreased. Other than doing occasional home or community improvement projects, walking around the neighbourhood or to the pub, you get no regular exercise. 
Instructions to Physician: You are about to meet Dana Jones. The patient does not have an FP, but Dr Rontowski, at a nearby walk-in-clinic, sent him/her for a chest X-ray last week. The results showed a suspicious mass, prompting Dr Rontowski to send Dana for a PET-CT scan. Dana is here now for these results. After reviewing this patient's chart and images/report, discuss your diagnosis and treatment plan with the patient. Answer any questions the patient may have. During this encounter, please do and say what you would do and say normally in practice.

\section{Chart Note:}

Dana Jones

S: $67 \mathrm{y} / \mathrm{o}$

2 wk Hx productive hemoptysis and SOB; brown phlegm

50-75-pack-year smoker; alcohol 7-14/wk, no street drugs

Meds: None

Allergy: NKA

PMH: None

Fam Hx: Father died bronchogenic CA 50s; mother died MI 90 y/o; sibling died bronchogenic CA 70 y/o; sibling died MI 72 y/o; sibs 69 and 73 y/o A\&W

O: T 38 BP 120/80 HR 72 reg

A: bronchogenic CA, bronchitis, COPD

P: chest X-ray f/u PET-CT

\section{Images:}

See four image sets at: http://gamma.wustl.edu/pt096te163.html

Radiopharmaceutical: $15 \mathrm{mCi}$ F-18 Fluorodeoxyglucose i.v. Findings: "The PET/CT images demonstrate intense FDG uptake within the right upper lobe mass, which extends centrally to involve the right hilum. No distant metastasis seen. The chest radiograph (PA) shows a large right upper lobe mass concerning for a primary lung neoplasm. The contrasted CT confirms the right upper lobe mass which measures $8.0 \mathrm{~cm} \times 5.2 \mathrm{~cm}$ and also demonstrates right hilar adenopathy $(1.1 \mathrm{~cm})$. Consistent with non-small cell lung cancer of the right upper lobe with pulmonary vein extension." 124

\footnotetext{
${ }^{124}$ McDaniel, Brock and Farrokh Dehdashti. Teaching File Case number: pt 096. Mallinckrodt Institute of Radiology, Washington University. Web. 31 Dec 2012. <http://gamma.wustl.edu/pt096te163.html>
} 


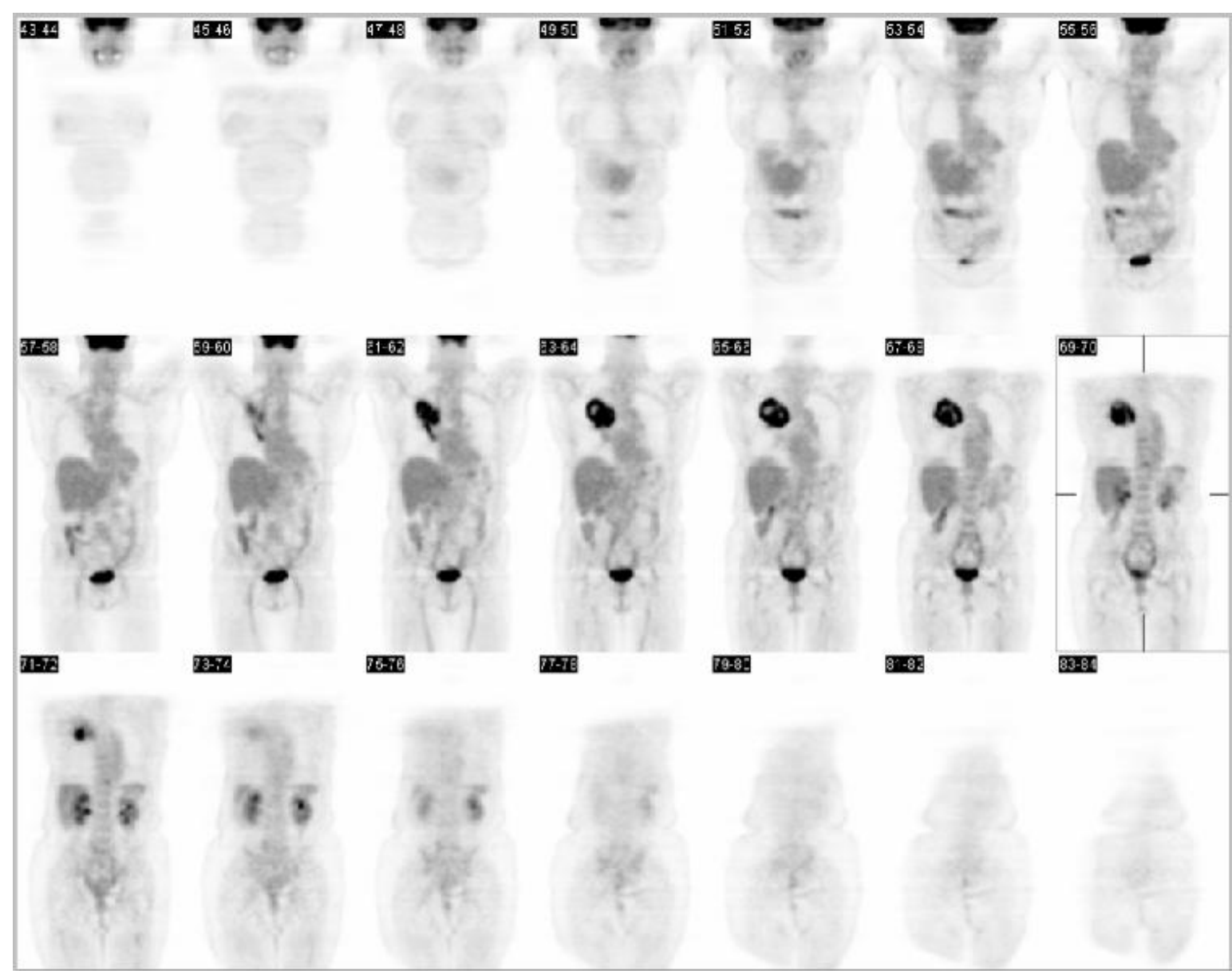



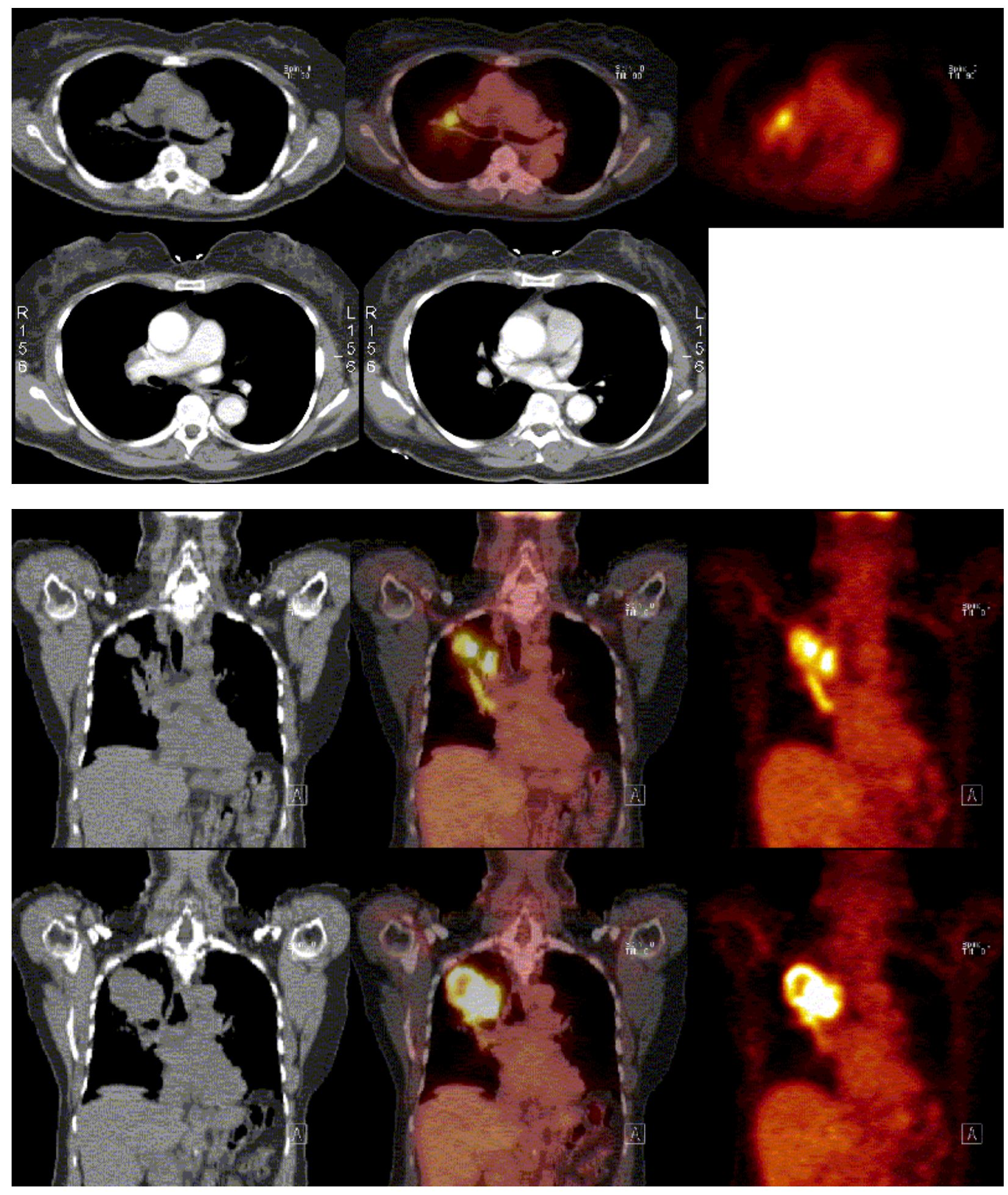


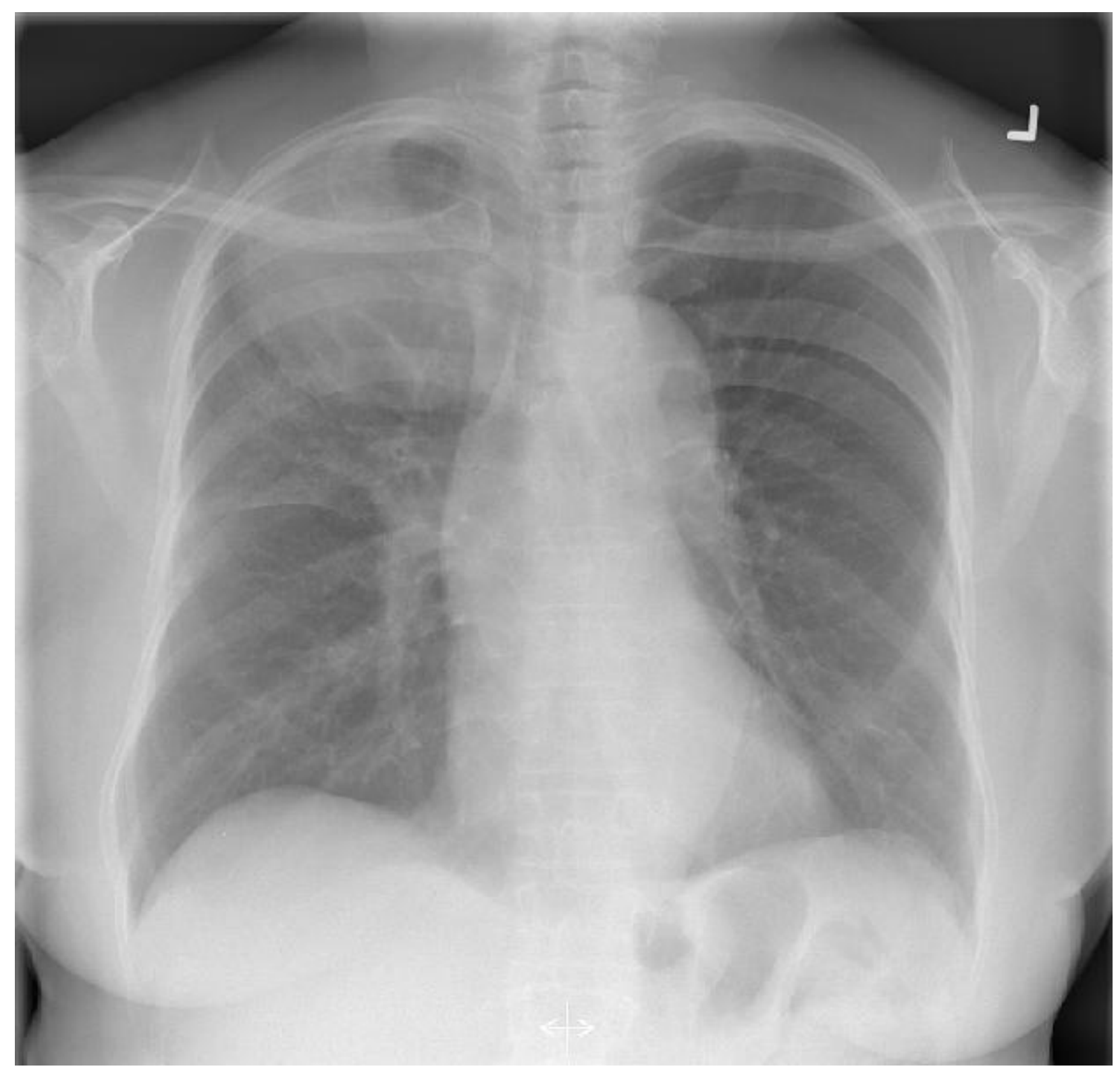




\section{Cardiac Role (benign):}

Intent: These are physician-driven, conversational encounters. If the physician says something that is not clear to you, you might ask for clarification. If the physician offers to show you the images, you will be interested. You will talk about the imaging experience and/or your fears around your current health situation, only if the physician provides the opportunity.

Patient: Skyler Hughes, age 69

Opening Line: Hello, doctor (or similar greeting).

Prompt (Things the patient might ask): Can you show me the pictures? They might help me understand better.

Note: This prompt should only be given if yours is the second role a physician is seeing and only if s/he has not already offered to show you the images. If the physician refuses, allow the encounter to end naturally.

Medical History: In the last six months, you've noticed an increasing shortness of breath and occasional mild chest pain (2/10) when you do normal activities, like walking, climbing stairs, or sports. The symptoms resolve on their own in two or three minutes. Although you didn't think it was worth making an appointment to see your doctor, you mentioned it during a recent routine physical. Dr Chan said that what you were describing was "atypical chest pain" and she sent you for a "test," a coronary CT angiogram (CTA) to rule out anything serious.

Imaging Experience: You weren't sure what to expect when you arrived at the hospital at the appointed time. You felt a little nervous, because of the potential results, but you had confidence in the professionals doing the procedure. You were asked to change into a hospital gown and then enter the CT imaging suite. The machine occupied most of the very cold room. You were asked to lie down on the bed extending from the machine. Once in position, EKG leads were attached to your chest, and an IV line was inserted into your arm; the technician explained that the IV would introduce a contrast medium used to enhance the images. You experienced a flush of heat and a metallic taste in your mouth when the IV started, but these symptoms quickly passed. The technician explained that he would need you to remain completely still throughout the exam, and that he would ask you to hold your breath for intervals during the procedure. He then asked you to raise your arms above your head, which was somewhat uncomfortable. The bed emitted some whirring sounds as it moved into the scanner. As soon as you entered the CT tube, you felt claustrophobic and wanted out. Even though your head was almost outside the machine, you had to work hard not to panic. At various intervals, the technician asked you to hold your breath for about 20 seconds (it seemed like a lot longer!); this was difficult, as you were panic-breathing. It took a lot of willpower, but you tried to comply. Eventually, you were released from the machine, and asked to wait for confirmation that the images were clear, before you could leave. In a few minutes, you were told that you could go and the IV line was removed. 
When you changed back into your street clothes, you looked at your watch and realized that the whole thing had only taken twenty minutes. You laughed off your panic and realized that the procedure had been fast, simple, and painless.

\section{Further information to help explain the imaging process to the SP:}

For a short video on the patient experience of the CT angiogram procedure, refer to:

http://www.youtube.com/watch?v=aKDHrNY1LKw

This site has a short educational video with more detailed information:

http://www.youtube.com/watch?v=2iqmWFPYDtY

\section{Medications: None.}

Affect: You're generally a relaxed and upbeat person. Although you're curious about what's causing your symptoms, you're not worried. You respect doctors ("They know a lot more than me!') and are confident that this physician will tell you anything you need to know. You'll accept whatever the doctor says, even if you may not understand it all. The imaging might help the physician explain the problem. Although you want to understand the findings, you're more interested in knowing, in simple terms, what's wrong and what can be done about it. If frustrated, you might just tune out. If a strong therapeutic alliance is formed, you may be more inclined to talk about your imaging experience and fears (e.g., the panic during imaging, "What's my doctor worried about?"), given the opportunity.

Allergies: No known allergies.

Immunizations: Up to date.

Personal History: You're a retired school janitor. You're smart and probably could have done more with your life, but that would have required more effort and education; you dropped out of high school. You were happy with your job, and enjoyed talking to the kids and watching them grow up. You were always very careful with your money, and are comfortable in your retirement. You never married or had a family, but you don't feel like you've missed much. You've got lots of friends with whom you meet most days at Tim Horton's. Sometimes the group of you will sit there all morning, talking about world politics, sports, and TV shows.

Family History: You grew up in rural Ontario, and your older brother still works the family farm with help from his children and grandchildren. Your father died of lung cancer when he was 78. You don't remember him without a cigarette dangling from his mouth, and the cancer was advanced when it was discovered; he didn't suffer very long. Your mother stayed in the family home where she was looked after by other family members until she died four years ago at age 94 . She remained mentally alert, but her body slowly wasted away; as far as you're concerned, she died of "old age." Your brother, Tim, is 75 and in excellent health. You also have a 72-year-old sister, Eileen, who lives close to your brother. Although generally healthy, she 
suffers from some kind of arthritis; you don't know the details. You stay in touch by phone once a week, but don't see each other very often. You have lots of aunts, uncles, and cousins, but you're not in touch with them and only see them at family reunions every few years. You do know that people in your family tend to be very healthy and live a long time; one of your aunts is 106 !

Lifestyle: You've never smoked; and when you saw what it did to your father, you're glad you never took up the habit. Once or twice a week, you go with your friends to a bar in the evening where you'll have one or sometimes two beers; the only time you drink heavily is at family occasions, like a wedding or funeral. You've never done street drugs; they didn't interest you, but as a school janitor, you felt it important to set a good example. You think that the physical activity of your work kept you healthy, and now that you've retired, you've taken up bowling and curling. You've started doing jigsaw and other puzzles, to keep mentally active, and have found them quite enjoyable. Since retiring, you spend a lot of time at Tim Horton's, so you often eat there; you like their donuts, crullers, and lunchtime sandwiches. You don't really cook ("It's hardly worth it for one person."), and rely on prepared foods from the supermarket buying whatever's cheapest. Because you live alone, you're conscious of staying health; there's no one to take care of you if something happens. Eventually, you expect to move back to the family farm where there are younger family members to care for you, but you're not looking forward to moving away from your friends and the life you've established in the city. You get annual medical check-ups, and, until now, things have been going well. 
Instructions to Physician: You are about to meet Skyler Hughes. Skyler had a cardiac CTA last week and is here for the results. His/her FP, Dr Chan, thought you might be better qualified to discuss the results with this patient. After reviewing Skyler's chart and CTA results, discuss your diagnosis and treatment plan with the patient. Answer any questions the patient may have. During this encounter, please do and say what you would do and say normally in practice.

\section{Chart Note:}

\section{Skyler Hughes}

S: $69 \mathrm{y} / \mathrm{o}$

6 mo Hx exertional dyspnea and atypical chest pain 2/10; resolves 2-3 mins

Non-smoker, regular alcohol intake + binges, no street drugs

Meds: None

Allergy: NKA

PMH: None

Fam Hx: Father died lung CA 78 y/o; mother died 94 y/o cause unknown; brother 75 y/o A\&W; sister 72 y/o arthritis

O: T 37 BP 120/80 HR 72 reg

\section{A: LHAS, lipoma}

P: CTA

\section{Images:}

See eight images at:

http://rad.usuhs.mil/medpix/tf_case.html?mode=case_viewer\&case_prog=cow_image.html\&th=$1 \&$ quiz=yes\&pt $\mathrm{id}=12100 \& \mathrm{imid}=0 \&$ imageid $=43802 \&$ week $=\& \max =\&$ skiprows $=\& \operatorname{maxcases}=\&$ skipcase $\underline{\mathrm{s}=\& \text { this_week=\&conf=\#clinical }}$

Findings: "Axial noncontrast enhanced and post-contrast (IV) images through the heart demonstrate low attenuation of the entire right atrial septum consistent with lipomatous hypertrophy of the interatrial septum."

\footnotetext{
${ }^{125}$ Mullins, Andrew. Case 12100. MedPx TM Medical Image Database, Atlas, and Teaching File, Department of Radiology and Rodiological Sciences, Uniformed Services University (Bethesda, MD). Web. 31 Dec 2012.

<http://rad.usuhs.mil/medpix/tf_case.html?mode=case_viewer\&case_prog=cow_image.html\&th=$\underline{1 \& \text { quiz }=\text { yes \&pt } \_\mathrm{id}=12100 \& \mathrm{imid}=0 \& \mathrm{imageid}=43802 \& \text { week }=\& \max =\& \text { skiprows }=\& \text { maxcases }=\& \text { skipcases }=\& \text { this } \_\mathrm{w}}$ $\underline{\text { eek }=\& \text { conf }=\# \text { clinical }>}$
} 


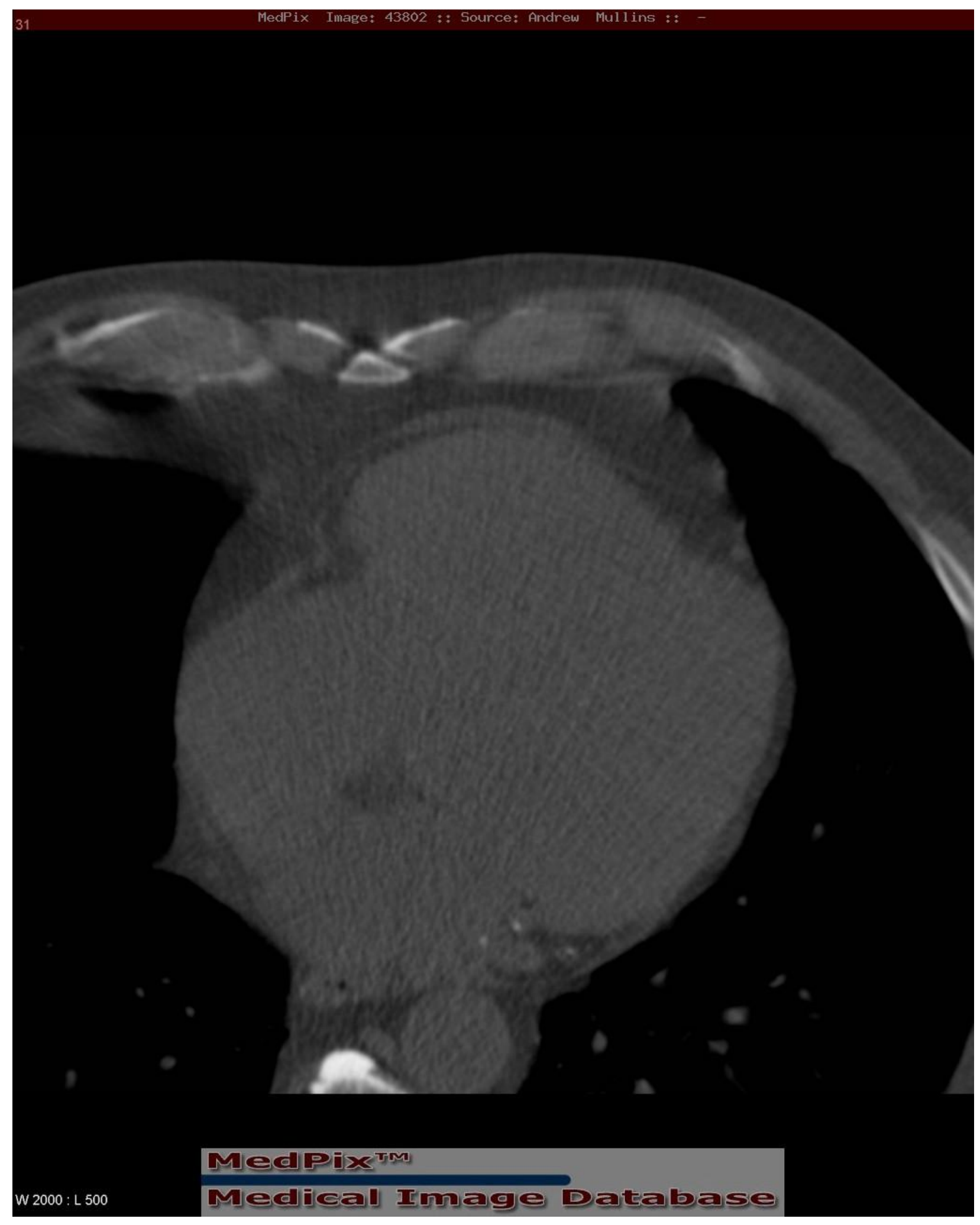




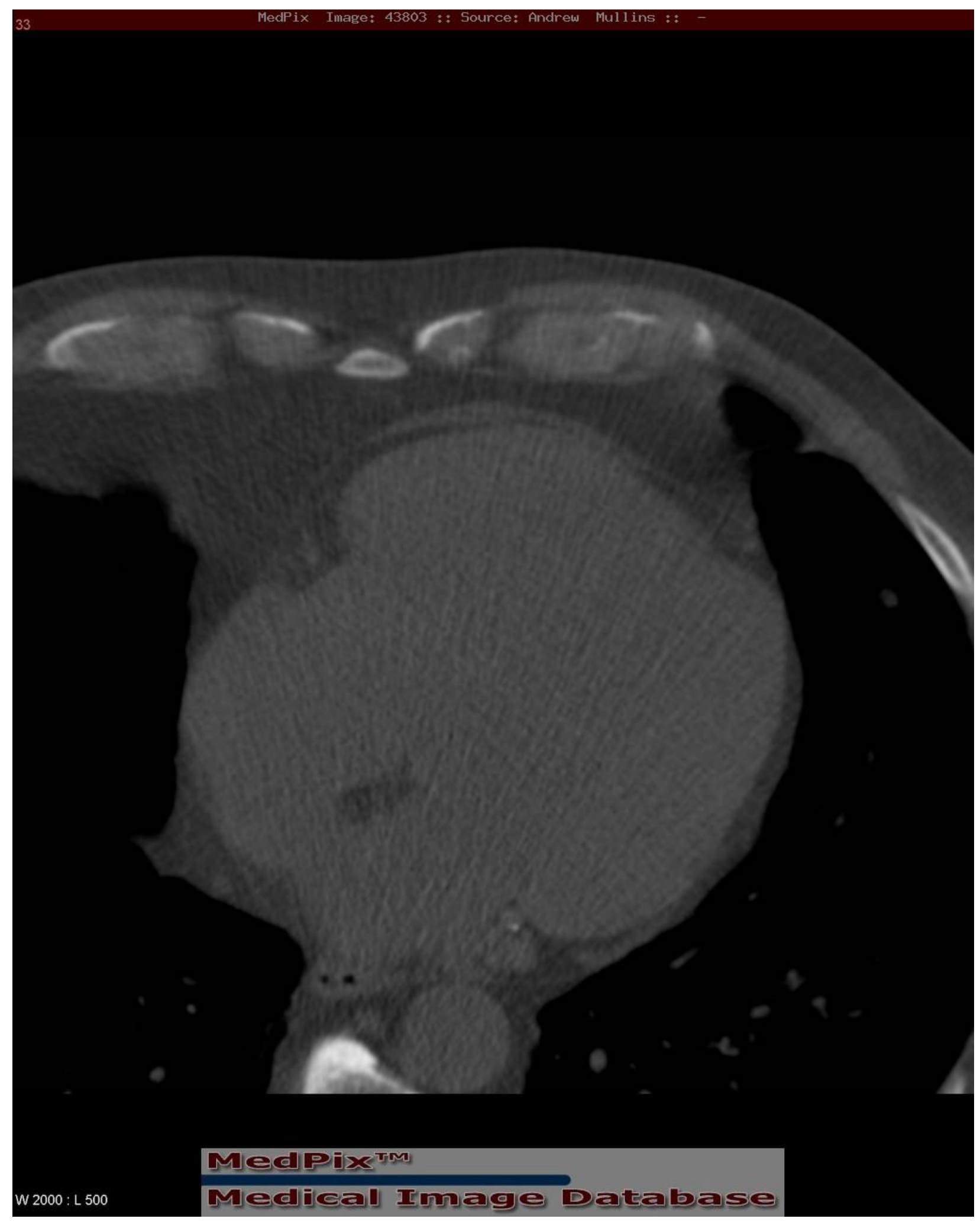




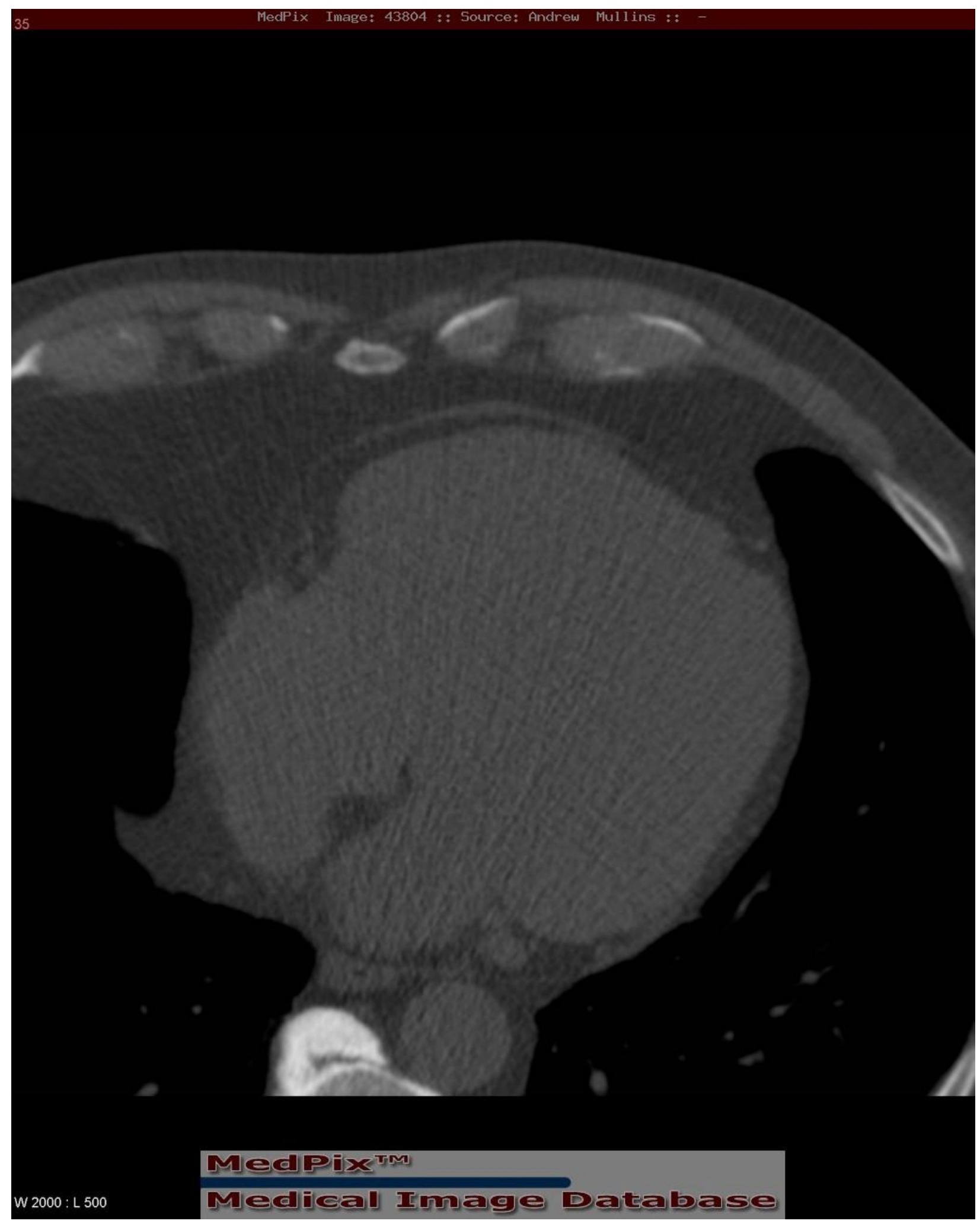




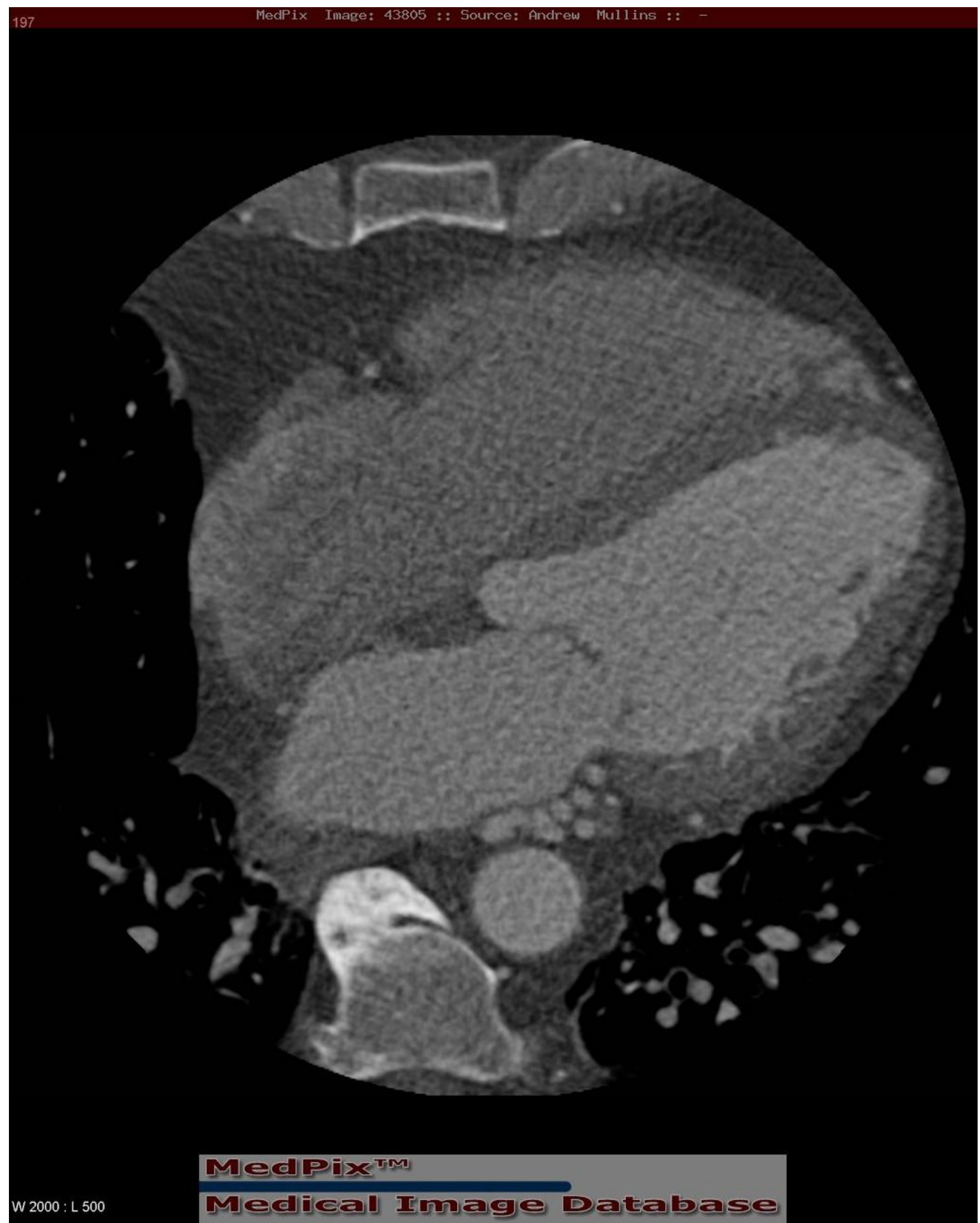




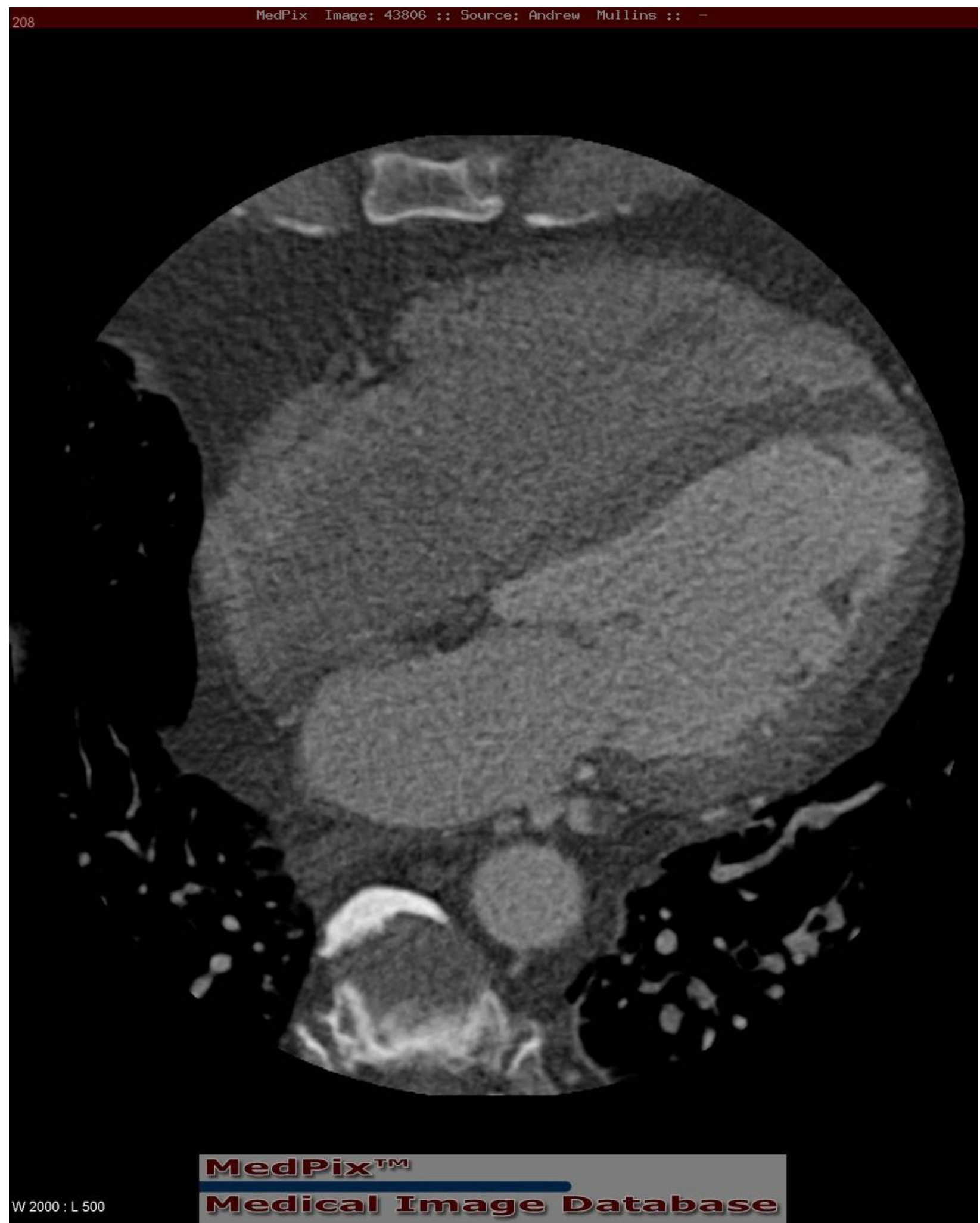




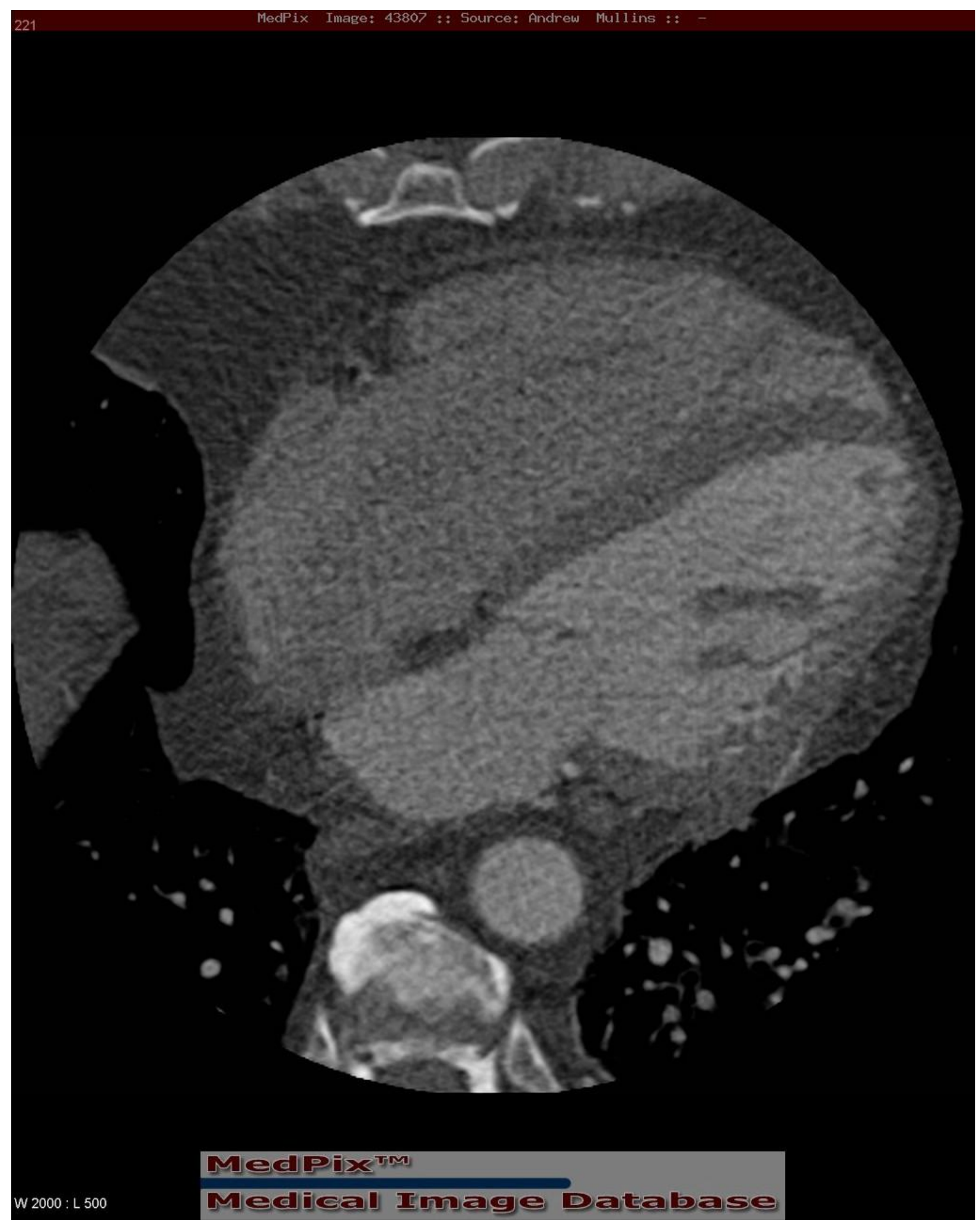




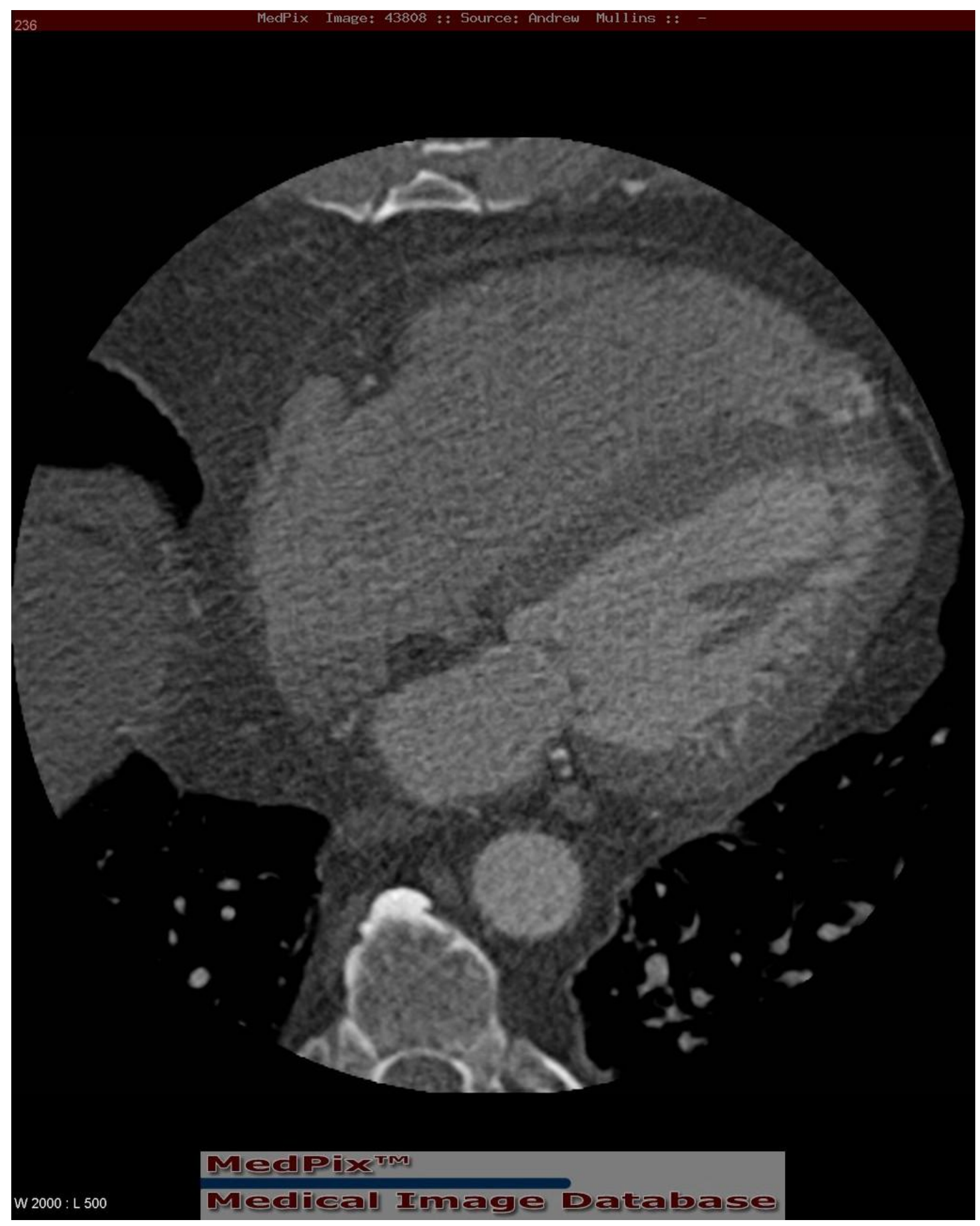




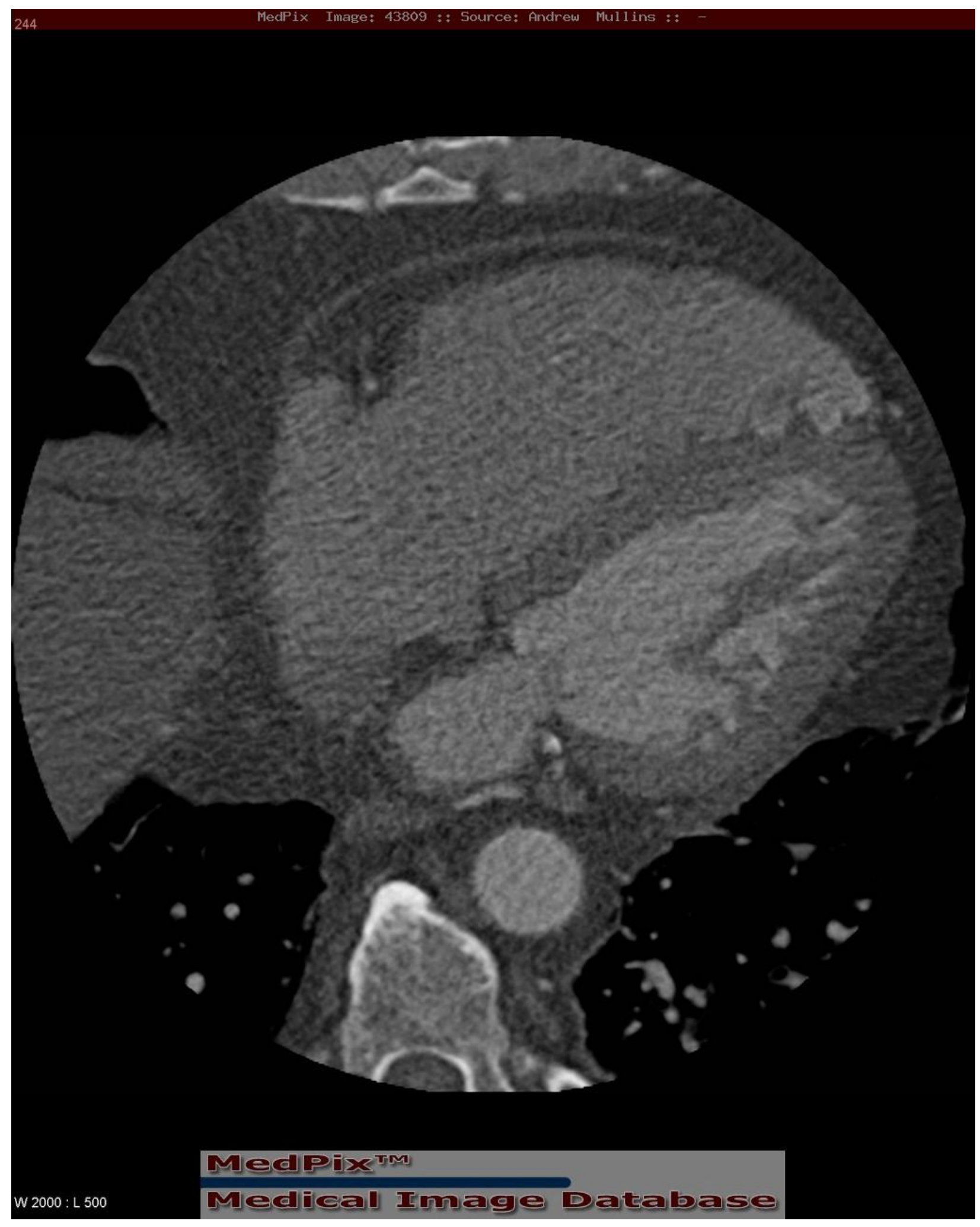




\section{Cardiac Role (findings):}

Intent: These are physician-driven, conversational encounters. If the physician says something that is not clear to you, you might ask for clarification. If the physician offers to show you the images, you will be interested. You will talk about the imaging experience and/or your fears around your current health situation, only if the physician provides the opportunity.

Patient: Chris Anderson, age 72

Opening Line: So, how bad is it?

Prompt (Things the patient might ask): May I see the angiogram?

Note: This prompt should only be given if yours is the second role a physician is seeing and only if s/he has not already offered to show you the images. If the physician refuses, allow the encounter to end naturally.

Medical History: Six months ago, after a routine check-up by your FP, Dr Cummings, you were diagnosed with high cholesterol (6.2) and high blood pressure (150/90), although both seem well controlled on the current medication (see below). While you're not sure what your present cholesterol reading is, your blood pressure is now 130/84.

Three weeks ago, you started noticing an uncomfortable squeezing sensation and heavy pressure ("like an elephant") in your chest. Sometimes, this sensation occurs after mild exertion, like climbing the stairs or walking a couple of blocks, but it has also occurred at rest or after dinner. At first, you thought it was just indigestion, but the pain started occurring more frequently, so your spouse suggested making an appointment with Dr Cummings. You saw Dr Cummings two weeks ago and he sent you to a cardiologist, Dr Smithson. You saw Dr Smithson last week, and she immediately booked you for an angiogram.

The attacks last up to ten minutes, are sometimes associated with breathlessness, and sometimes refer pain to the left arm or jaw. The pain is severe, an $8 / 10$ on the pain scale. Initially, you experienced an attack every two or three days, but now they occur two or three times each day.

Imaging Experience: The imaging was done in a ward where at least a dozen other patients were also having angiograms. You changed into a hospital gown and were moved to a hospital bed and put on an IV drip. The nurse assisting with the procedure anesthetized the inside of your left thigh and inserted a needle into an artery, through which a catheter was threaded up into the heart. X-ray captures of dye diffusing through the catheter were fed to a monitor for a doctor to review. The doctor was unfamiliar to you and did not introduce himself; you didn't even realize the doctor was present until he said, "Oh yeah, there's the heart." The assisting nurse invited you to turn your head to see the monitor, located behind you and to the left, but you kept your eyes closed throughout the procedure and refused to look. The doctor's only comments during the imaging procedure were non-committal: "Oh yeah, look, yea, uh-hmm... okay... yup..." then 
"That's fine. That's all we need." Then the catheter was removed and you were transferred to a large recovery room, where a weight was placed on your leg for about 45 minutes to prevent bleeding. An attendant stayed with you during this time, but you were the last patient of the day, the only one left in recovery, and it was apparent that the attendant wasn't happy at having to stay; it was the end of shift and his colleagues were all filing out. While you were in recovery, the doctor who had done the imaging arrived and informed you that there were several arterial blockages around the heart and that surgery might be necessary, but that details would be discussed with your regular doctor. There was no opportunity to ask questions. The information provided was enough to further frighten you, but did nothing to alleviate your fears. While the whole imaging procedure was scary, it was not painful. Although your fear was apparent, it was not addressed by anyone attending to you.

\section{Further information to help explain the imaging process to the SP:}

For an animated description of an angiogram procedure, please refer to: http://www.nhlbi.nih.gov/health/health-topics/topics/ca/during.html

Here are some photos of a typical angiographic suite and machinery: http://www.radiologyinfo.org/en/photocat/gallery2.cfm?pid=1\&pg=angiocath

\section{Medications:}

\begin{tabular}{|l|l|l|l|}
\hline Medication & Indication & Dosage & Duration \\
\hline Hydrochlorothiazide & Hypertension & $25 \mathrm{mg}$ once per day & 6 months \\
\hline Ramipril (Altace) & Hypertension & $2.5 \mathrm{mg}$ once per day & 6 months \\
\hline Lipitor (Atorvastatin) & Hyperlipidemia & $20 \mathrm{mg}$ once per day & 6 months \\
\hline Aspirin & prophylactic & $81 \mathrm{mg}$ once per day & 6 months \\
\hline Nitroglycerin & Angina & $0.4 \mathrm{mg}$ PRN & 2 weeks \\
sublingual spray & & $\begin{array}{l}1-2 \text { sprays; may be } \\
\text { repeated twice after 5- } \\
\end{array}$ & \\
& & minute intervals & \\
\hline
\end{tabular}

Affect: You are a take-charge kind of person, but the current health situation is beyond you. It's up to your body and the doctors to decide what happens next, and this loss of control is causing great anxiety. The idea that something might be wrong with your heart, especially given your family history, is overwhelming. You're not sure if you're going to be okay and expect the worst. "Am I going to have a heart attack?" "Is this going to kill me?"

The imaging procedure was not explained in advance; you were just told to show up at the hospital at an appointed time for the procedure. Although the medical staff explained the procedure step-by-step as they were doing it, you remained extremely anxious throughout, keeping your eyes closed and trying to breathe normally. It was a bad experience and has left you 
feeling shaken and anxious about what might come next. "So, what can we do about this?" "Are there any other options?"

You are assertive, but not aggressive. You are used to being in control; the only way you can restore some measure of control is by gathering more information. Although this is a physiciandriven interview, you will ensure that you have a thorough understanding of the findings and treatment options. You will try to retain direct eye contact throughout the interview. If frustrated, you might attempt to pull papers from the physician's hand. If a strong therapeutic alliance is formed, you may feel calmer and be more inclined to open up about your imaging experience and fears (e.g., mortality issues), given the opportunity.

Allergies: No known allergies.

Immunizations: Up to date, including flu shot.

Personal History: You worked as an accountant from age 23 until you retired at age 65, ending your career at Deloitte and Touche. You are financially secure, with a good pension, and have lived in the same house with your partner for over thirty years. You married your high-school sweetheart at 21, divorced at 28, and remarried at 32 to your current spouse. You had a daughter with your first partner and a son and daughter by your second partner. The children have left home, two are married and one has children; they are generally financially independent. You are close to all of your children, although there is sometimes tension between the children from the two marriages, as well as between your first and second partners. You have stayed in touch with some of your work colleagues and occasionally take overflow accounting work. Although some of your colleagues golf, you've never been so inclined. In fact, you prefer quiet pastimes like galleries, museum, later-life learning classes, and books. Although you were very frightened during the imaging experience, you are interested in finding out the details regarding your condition and what can be done.

Family History: Your father died of a heart attack at age 76; your mother died from a stroke at 78. You have an older brother, Jerry, who lives in Vancouver. You don't talk often, but you know that Jerry had a mild heart attack three years ago, when he was 75.

Lifestyle: You have never smoked or done drugs. You enjoy a glass of wine with dinner, maybe 3-5 times per week. Since retiring, you and your partner enjoy making meals together and, especially since your diagnosis of high blood pressure and high cholesterol six months ago, you are conscious about making healthy, low sodium, dietary decisions. Once or twice a week, your partner persuades you to get out for a gentle stroll, but that's all the exercise you get. You know that exercise is important given your health conditions, but you just aren't interested. 
Instructions to Physician: You are about to meet Chris Anderson. Chris had a cardiac angiogram last week and is here for the results. His/her cardiologist, Dr Smithson, was called into an emergency and asked if you could speak with this patient. After reviewing Chris's chart and imaging, discuss your diagnosis and treatment plan with the patient. Answer any questions the patient may have. During this encounter, please do and say what you would normally do and say in practice.

\section{Chart Note: Chris Anderson}

S: 72 y/o

3 wk Hx undiagnosed chest pain 8/10; ptn describes pain as heavy pressure or squeezing; exertional and postprandial; attacks up to $10 \mathrm{mins}$, 2-3 per day; assoc breathlessness, referred pain left arm and jaw Regular alcohol intake, non-smoker, no street drugs

Meds: Nitro sublingual spray $0.4 \mathrm{mg}$ PRN 2 wks

Hydrochlorothiazide $25 \mathrm{mg}$ QD 6 mo

Ramipril 2.5 mg QD 6 mo

Lipitor $20 \mathrm{mg}$ QD 6 mo

ASA $81 \mathrm{mg}$ QD 6 mo

Allergy: NKA

PMH: HCL X 6 mo

HTN X 6 mo

Fam Hx: Brother MI 75 y/o; father died MI 76 y/o; mother died CVA 78 y/o

O: T 37 BP 130/84 HR 75 reg

A: unstable angina

P: urgent angiogram

Images:

See three images at: http://gamma.wustl.edu/mi016te157.html

Radiopharmaceutical: 2.7 mCi Tl-201 chloride, i.v. (rest); and 21.6 mCi Tc-99m sestamibi, i.v. (stress)

Findings: "No focal myocardial perfusion defects are seen although there is mild heterogeneity of radiotracer distribution. There is mild left ventricular enlargement. Gated Tc-99m sestamibi images demonstrate moderate diffuse hypokinesia. Coronary angiography demonstrates highgrade $(>90 \%)$ stenosis in both the left anterior descending and right coronary arteries. Threevessel coronary artery disease with balanced ischemia and cardiomyopathy.",126

\footnotetext{
${ }^{126}$ Chesnut, Jeff \& Robert Gropler. Teaching File Case number: mi016. Mallinckrodt Institute of Radiology, Washington University. 15 Dec 1998. Web. 31 Dec 2012. 〈http://gamma.wustl.edu/mi016te157.html〉.
} 

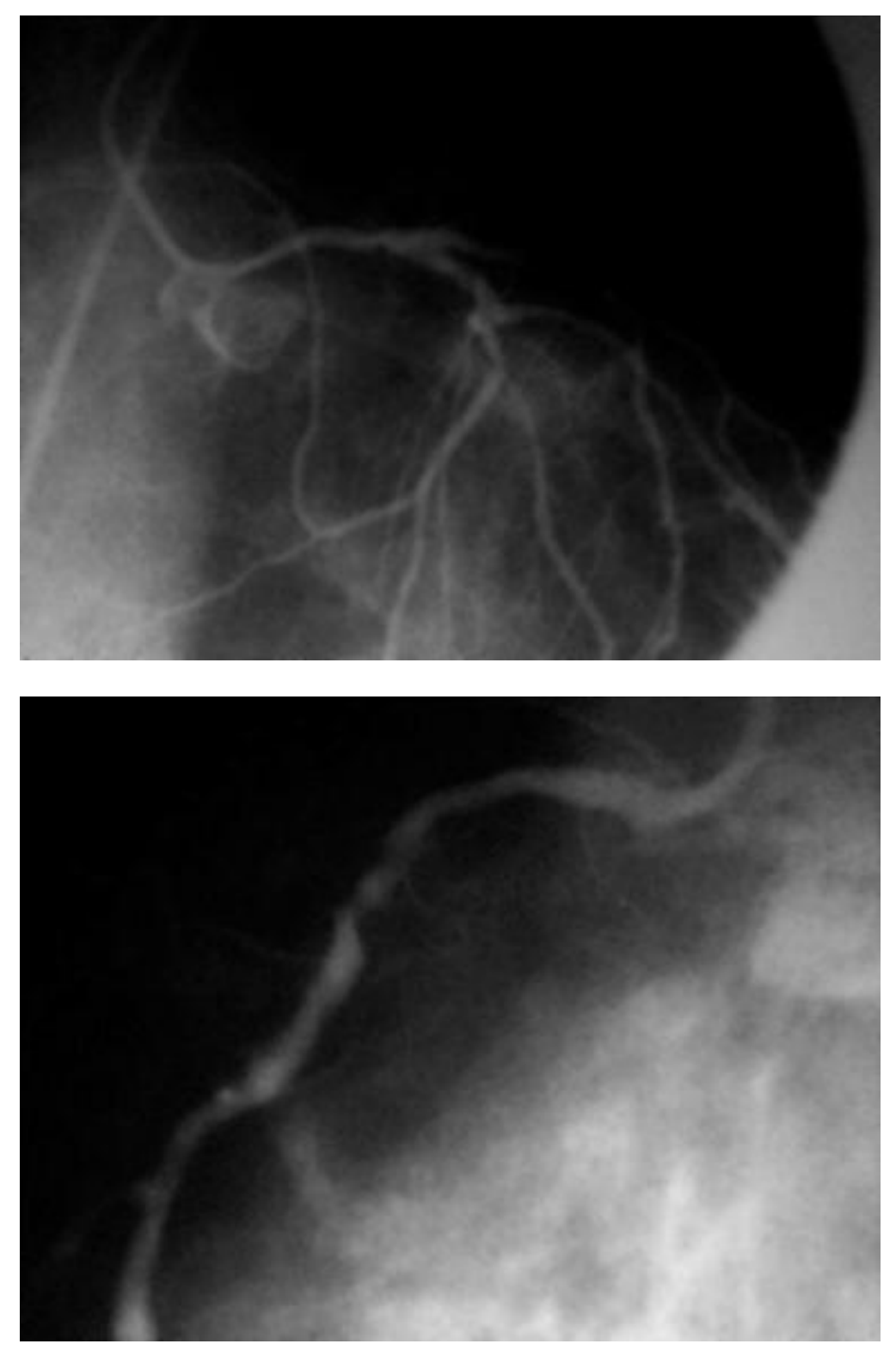


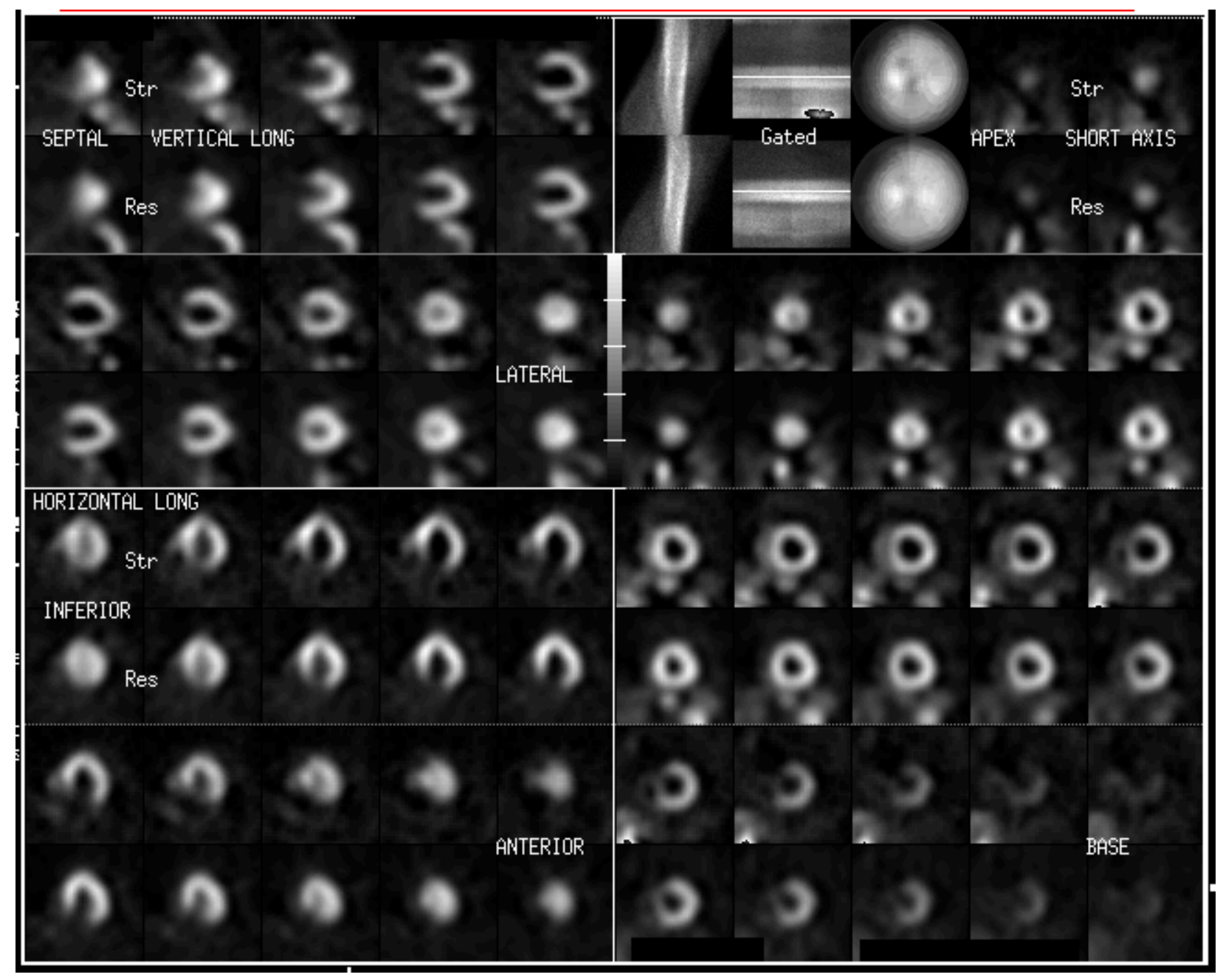




\section{Appendix E: Observation Protocol}

This Observation Protocol has been drafted to ensure clarity and focus with regard to information gathering during simulated patient-physician encounters. These encounters will be observed in real time, but also video recorded for further analysis. The location for these encounters, Ryerson's Nursing Lab at 415 Yonge St., allows for unobtrusive real-time observation through one-way glass, as well as access to the necessary video recording equipment.

During the discussion of Informed Consent, both the simulated patients and physicians involved in this study will be informed that:

- their encounters will be observed and recorded solely for research purposes;

- by signing the consent, they agree to the possibility that things they say or do during these encounters may be published in the study, but not attributed to them by name;

- these recordings, and transcripts derived from them, will be kept secure and confidential for a period of five years and then destroyed;

- should they choose to withdraw from the study, this material will be destroyed immediately and not entered into the data set.

For purposes of documentation, the researcher will note the names of the physician, the simulated patient, and which encounter is being observed, however, all data files will immediately be assigned numbers to ensure participant anonymity. Acknowledging both verbal and non-verbal communication, the following loosely structured protocol questions will be considered during real-time observation of simulated patient-physician encounters:

- Overall, is the verbal exchange conversational or is one party dominating the discourse?

- Is the physician's voice directed toward the simulated patient or the medical image?

- Is the physician using lay language or medical terminology?

- Is the physician checking to ensure simulated patient understanding or inviting questions from the simulated patient?

- Does the simulated patient have opportunity to ask questions and do they seem to understand the responses?

- Does the physical space between the physician and the simulated patient change during the course of the encounter? If so, who initiated this change and was there a response, either verbal or non-verbal?

- Is eye contact with the simulated patient primary or secondary to the focus on the medical image?

- Does the simulated patient appear comfortable in the encounter or does he or she seem confused or agitated?

- What is the focus of the simulated patient's gaze; the physician (regardless of that party's focus) or the medical image? 
More tightly structured observation protocol questions will be adopted for analysis of the recorded data, such as:

- How long was the encounter and what is the variation in time between different physicians approaching the same encounter?

- If medical language was used without explanation or clarification, how many times did this occur?

- Did the simulated patient ask questions that went unanswered, and if so, how many?

- How many minutes was the physician focussed on and making eye contact with the simulated patient, and how much time was focussed on the medial image?

- How many minutes was the simulated patient focussed on and making eye contact with the physician, and how much time was focussed on the medical image?

The researcher's observations of the encounters will be augmented with information derived from post-encounter interviews with both the physicians and the simulated patients. 


\section{Appendix F: Post-Encounter Semi-Structured Interview Questions}

\section{Semi-Structured Interview Questions for Physicians (post-encounter):}

\section{Introduction:}

Thank you for agreeing to participate in this research study. Before we discuss your encounter, do you have any questions?

\section{Questions:}

1) Please tell me about your encounter with this patient.

2) In this encounter, did you say and do everything that you normally would? Upon reflection, is there anything you wish you had said or done that you think you neglected?

3) How would you assess your conversation with this patient? Do you think that you used language appropriate for this patient? In what ways did you, or did you not? How did you determine if the patient clearly understood?

4) Do you think this patient had adequate opportunities for clarification or to ask any questions he or she might have had? (Explain)

5) Where was your primary focus during the encounter/interview? (Explain)

6) Did the philosophy of patient-centred care inform your encounter; if so, how?

7) Were you comfortable with the physical space between yourself and the patient? Did you do anything to change the physical space and if so, why?

8) Do you think that you had good eye contact with the patient during this encounter?

9) Do you think the patient was generally comfortable throughout this encounter or were you aware of his or her discomfort? If you think the patient seemed uncomfortable, do you know why? Did you do anything to try to alleviate his or her discomfort?

\section{Closing:}

Is there anything else you'd like to tell me about your experience? Do you have any other questions?

Thank you. 


\section{Semi-Structured Interview Questions for Simulated Patients (post-encounter):}

\section{Introduction:}

Thank you for agreeing to participate in this research study. Before we discuss your encounter, do you have any questions?

\section{Questions:}

1) Please tell me about your encounter with this physician. Overall, how did you feel during this encounter?

2) Was the language this physician used appropriate and understandable for you as a patient? In what ways was it, or was it not? Did the encounter feel one-sided with closedended questions, or was it more open-ended and conversational?

3) Did you have adequate opportunities to ask for clarification or to ask any questions you had? Were the physician's answers to your questions understandable?

4) Where was your primary mental focus during the interview? What is your sense of where the physician's primary mental focus was during the interview?

5) Were you comfortable with the physical space between yourself and the physician? Did you do anything to change the physical space and if so, why?

6) Do you think that you had good eye contact with the physician during this encounter?

7) Did you feel comfortable throughout this encounter? If you felt uncomfortable, do you know why? What did you do to try to alleviate your discomfort?

\section{Closing:}

Is there anything else you'd like to tell me about your experience? Do you have any other questions?

Thank you. 


\section{Works Cited}

Ackerknecht, Erwin H. A Short History of Medicine, Revised. New York: The Ronald Press, 1968. Print.

Ackerman, Diane. A Natural History of the Senses. New York: Random House, 1991. Print.

Alfus Healthcare Advocacy Program. University of Miami. n.d. Web. 18 July 2014. $\langle$ http://continue.miami.edu/en/packagedetail.aspx? $\mathrm{p}=100>$

American Society of Clinical Oncology (ASCO). What is Radiation therapy? 2005-2015. Web. 19 July 2014. <http://www.cancer.net/navigating-cancer-care/how-cancertreated/radiation-therapy/what-radiation-therapy $>$.

Anderson, Robert E., Rolla B. Hill, and Charles R. Key. The Sensitivity and Specificity of Clinical Diagnostics During Five Decades: Towards an Understanding of Necessary Fallibility. Journal of the American Medical Association. 261.11 (17 March 1989): 16101617. Web.

Anthony, Shawn G., Luciano M. Prevedello, Maria M. Damiano, Tejal K. Ghandi, Peter M. Doubilet, Steven E. Seltzer, and Ramin Khorasani. Impact of a 4-year Quality Improvement Initiative to Improve Communication of Critical Imaging Test Results. Radiology. 259.3 (June 2011): 802-807. Print.

Argyle, Michael, Veronica Salter, Hilary Nicholson, Marylin Williams, Philip Burgess. The Communication of Inferior and Superior Attitudes by Verbal and Non-verbal Signals. British Journal of Social and Clinical Psychology. 9.3 (September 1970): 201-300. Web. DOI: $10.1111 / \mathrm{j} .2044-8260.1970 . t b 00668 . x$

Armstrong, David. Bodies of Knowledge/Knowledge of Bodies. Reassessing Foucault: Power, Medicine and the Body. Eds Colin Jones and Roy Porter. New York, NY: Routledge, 1994. 17-27. Print.

Aufderheide, Arthur. The Scientific Study of Mummies. Cambridge, UK: University of Cambridge Press, 2003.Web.

Austin, Cecil Kent. Medical Impressions of America. Boston Medical and Surgical Journal. 166 (1912): 799-804. Web.

American Social History Project/Center for Media and Earning. Baby Show Archive. (20022006). Web. 12 November 2008. <http://chnm.gmu.edu/lostmuseum/searchlm.php?function=find\&exhibit=baby\&browse= baby> 
Barnes, Sarah, Merryn Gott, Sheila Payne, David Seamark, Chris Parkeer, Salah Gariballa, and Neil Small. Communication in heart failure: perspectives from older people and primary care professionals. Health and Social Care in the Community. 14.6 (Marchh 2006): 482490. Web. <doi: 10.1111/j.1365-2524.2006.00636.x>

Baudrillard, Jean. The Precession of Simulacra. Simulacra and Simulation. Ann Arbor, MI: University of Michigan Press, 1994. 1-42. Print.

Baumann, Brigitte, Esther H. Chen, Angela M. Mills, Lindsey Glaspey, Nicole M. Thompson, Molly K. Jones, and Michael C. Farner. Patient Perceptions of Computed Tomographic Imaging and Their Understanding of Radiation Risk and Exposure. Annals of Emergency Medicine. 58.1 (July 2011): 1-7. Web. <doi:10.1016>

Bazar de la charité (2013). Web. 3 March 2014. <http://bazardelacharite.blog.free.fr/>

Beck, Stefan. Medicalizing culture(s) or culturalizing medicine(s). Biomedicine as Culture: Instrumental Practices, Technoscientific Knowledge, and New Modes of Life. Eds. Regula Valérie and Joseph Dumit. New York, London: Routledge, 2007. 17-33. Print.

Beecher, Henry, Raymond D. Adams, A. Clifford Barger,William J. Curran, Derek DennyBrown, Dana L. Farnsworth, Jordi Folch-Pi, Everett I. Mendelsohn, John P. Merrill, Joseph Murray, Ralph Potter, Robert Schwab, and William Sweet. A Definition of Irreversible Coma: Report of the Ad Hoc Committee of the Harvard Medical School to Examine the Definitioin of Brain Death. Journal of the American Medical Association. 5 August 1968. 205:6 (85-88). Web.

Benedict, A.L. The Art of Diagnosis. The Medical Age. 17 (25 September 1899): 861-690. Web.

Berlin, Leonard. Accuracy of Diagnostic Procedures: Has it Improved Over the Past Five Decades? American Journal of Roentgenology Diagnostic Imaging and Related Sciences. 188. 5 (May 2007). 1173-1178. Web.

Berliner, Howard S. A Larger Perspective on the Flexner Report. International Journal of Health Services. 5:4 (1975): 573-592. Web.

Bernat, James. Brain Death: Occurs Only With Destruction of the Cerebral Hemispheres and the Brain Stem. Archives of Neurology. 49.5 (May 1992): 569-570. Web.

<doi:10.1001/archneur.1992.00530290161027>.

---. A Defense of the Whole-Brain Concept of Death. Hastings Center Report. 28.2 (March-April 1998): 14-23. Web. <DOI: 10.2307/3527567>.

---, Charles M. Culver, and Bernard Gert. On the Definition and Criterion of Death. Annals of Internal Medicine. 94.3 (1981):389-394. Web. <doi:10.7326/0003-4819-94-3-389> 
Berrington de González, Amy, Mahadevappa Mahesh, Kwang-Pyo Kim, Mythreyi Bhargavan, Rebecca Lewis, Fred Mettler, and Charles Land. Projected cancer risk from computed tomographic scans performed in the United States in 2007. Archives of Internal Medicine. 169.22 (14-28 December 2009): 2071-2077. Web.

Beyer, Thomas, David W. Townsend, Tony Brun, Paul E. Kinahan, Martin Charron, Raymond Roddy, Jeff Jerin, John Young, Larry Byars, and Ronald Nutt. A combined PET/CT scanner for clinical oncology. Journal of Nuclear Medicine. 41:8 (August 2000): 136979. Web. 20 January 2013. <http://jnm.snmjournals.org/content/41/8/1369.long>

Birkelo, Carl, W. Edward Chamberlain, Paul S. Phelps, Percy E. Schools, David Zacks, and Jacob Yerushalmy. Tuberculosis Case Finding: A Comparison of the Effectiveness of Various Roentgenographic and Photofluorographic Methods. Journal of the American Medical Association. 133.6 (8 February 1947):359-366. Web. <doi: 10.1001/jama.1947.02880060001001>

Blaxter, Mildred. The Case of the Vanishing Patient? Image and experience. Sociology of Health and Illness. 31:5 (2009) 762-778. Web.

---. Diagnosis as Category and Process The Case of Alcoholism. Social Science and Medicine. 12 (19778):9-17. Web.

Bogdan, Robert. Freak Show: Presenting Human Oddities for Amusement and Profit. Chicago: University of Chicago Press, 1988. Print.

---. The Social Construction of Freaks. Freakery: Cultural Spectacles of the Extraordinary Body. Ed. Rosemary G. Thompson. New York: New York University Press, 1996. 23-37. Print.

Bonati, Maurizio Rippa. Some Traditions Regarding the Old Anatomy Theatre of Padua University. University of Padua. (n.d.). Web. 8 September 2011. <http://www.unipd.it/esterni/visiteweb/english/pagine/teatesto.htm>.

Breitsameter, Christopher. Medical decision-making and communication of risks: an ethical perspective. Journal of Medical Ethics. 36 (2010) :349-352. Web.

Brenner, David J. and Eric J. Hall. Computed Tomography-An Increasing Source of Radiation Exposure. The New England Journal of Medicine. 357.22 (29 November 2007): 2277-84. Web.

British Medical Journal. About the BMJ. 2011. Web. 1 March 2011. 〈http://www.bmj.com/about-bmj>

Brown, Lyn Mikel and Carol Gilligan. Raising their Voices: The Politics of Girls' Anger. Cambridge, MA: Harvard University Press, 1999. Web. 
Brown, Nik and Andrew Webster. New Medical Technologies and Society: Reordering Life. Cambridge, UK: Polity Press, 2004. Print.

Buckman, Robert. How to Break Bad New: A Guide for Health Care Professionals. Toronto: University of Toronto Press, 1992. Print.

Burri, Regula Valérie. Sociotechnical anatomy: Technology, space, and body in the MRI unit. Biomedicine as Culture: Instrumental Practices, Technoscientific Knowledge, and New Modes of Life. Eds. Regula Valérie Burri and Joseph Dumit. New York, London: Routledge, 2007. 109-121. Print.

Butterworth, J. Scott and Edmund H. Reppert. Auscultatory Acumen in the General Medical Population. Journal of the American Medical Association. 174.1 (3 September 1960): 3034. Web.

Canadian Broadcasting Corporation (CBC) News. Medical scan mistakes: what's behind the problems? 12 April 2012. Web. 12 April 2012. <http://www.cbc.ca/news/canada/story/2012/04/10/canada-medical-scans-problemsreview.html?cmp=rss $>$

Canadian Broadcasting Corporation (CBC) News. Finds your hospital or province's ER wait times to see a doctor: First time cross-country hospital data released. 18 September 2014. Web. 21 September 2014. <http://www.cbc.ca/news/find-your-hospital-or-province-s-erwait-times-to-see-a-doctor-1.2769552>.

Canadian Diagnostic. Fee Schedule. 2014. Web. 8 July 2014. <http://www.canadadiagnostic.com/content/about/fee-schedule.php〉.

Canadian Institute for Health Information (CIHI). Medical Imaging. 1996-2014. Web. 2 February 2014. <http://www.cihi.ca/cihi-extportal/internet/en/tabbedcontent/types+of+care/specialized+services/medical+imaging/ci $\underline{\text { hi010642> }}$

Canadian Medical Association Journal. About CMAJ. 2011. Web. 1 March 2011. <http://www.cmaj.ca/site/misc/about.xhtml $>$

Can Dogs Smell Cancer? Dir. Jemima Harrison. Passionate Productions, 2005. Documentary.

Cappabianca, Paolo and Francesco Magro. The lesson of anatomy. Surgical Neurology. 71.5 (May 2009): 597-599. Web. <doi:10.1016/j.surneu.2008.03.015>.

Caraballo, Damian E. How a Bowl of Beans and Rice, a Couple X-rays, and 8 Hours of Futility Taught Me to Trust My Own Intuition. Academic Emergency Medicine. 18.2 (February 2011) Web. <doi:10.1111/j.1553-2712.2010.00975.x> 
Carabine, Jean. Unmarried Motherhood 1830-1990: A Genealogical Analysis. Discourse as Data: A Guide for Analysis. Eds. Margaret Wetherell, Stephanie Taylor, and Simeon J. Yates. London, Thousand Oaks, New Delhi: Sage Publications, 2001. Print.

Carr, Herman. Field Gradients in Early MRI. Physics Today. $57: 7$ (July 2004): 83. Web. 20 January 2013 <doi: $\underline{10.1063 / 1.1784322>}$.

Cartwright, Lisa. Screening the Body: Tracing Medicine's Visual Culture. Minneapolis, MN: University of Minnesota Press, 1995. Print.

Catana, Ciprian, Daniel Procissi, Yibao Wu, Martin S. Judenhofer, Jinyi Qi, Bernd J. Pichler, Russell E. Jacobs, and Simon R. Cherry. Simultaneous in vivo positron emission tomography and magnetic resonance imaging. Proceedings of the National Academy of Sciences. 105:10 (2008): 3705-3710. Web. 12 January 2013. <doi: 10.1073/pnas.0711622105> .

Chalmers, John. Conan Doyle And Joseph Bell: The Real Sherlock Holmes, Surgeons' Hall Museums, Edinburgh. The Independent. 7 August 2006. Web. 6 august 2013. $<$ http://www.independent.co.uk/arts-entertainment/theatre-dance/reviews/conan-doyleand-joseph-bell-the-real-sherlock-holmes-surgeons-hall-museums-edinburgh$\underline{410821 . h t m l}>$.

Chesnut, Jeff and Robert Gropler. Teaching File Case number: mi016. Mallinckrodt Institute of Radiology, Washington University. 15 Dec 1998. Web. 31 Dec 2012. <http://gamma.wustl.edu/mi016te157.html $>$.

Clarke, Adele, Janet Shim, Laura Mamo, Jennifer Ruth Fosket, and Jennifer Fishman. Biomedicalization: Technoscientific Transformations of Health, Illness, and U.S. Biomedicine. American Sociological Review. 62: 2 (April 2003): 161-194. Web.

Cochrane, A.L. and L. Henry Garland. Observer error in the interpretation of chest films: an international investigation. The Lancet. 260.6733 (13 Sept 1952): 505-9. Web. <doi:10.1016/S0140-6736(52)90291-2>

College of Physicians and Surgeons of Ontario (CPSO). How Jumping to Conclusions -JTCcan Hinder Patient Care. n.d. Web. 〈http://www.cpso.on.ca/CPSO-Members/Resourcesfor-Physicians/Practice-Partner/Doc-Talk/How-Jumping-To-Conclusions>

Collins, Suzanne. Hunger Games. New York: Scholastic Press, 2008.

Community University Research Alliance (CURA). Living Archives on Eugenics in Western Canada. n.d. Web. 25 January 2014. 〈http://eugenicsarchive.ca/\#about-section〉 
Corbin, Alain. Introduction and Part I: The Perceptual Revolution, of the Sense of Smell on Trial. The Foul and the Fragrant: Odor and the French Social Imagination. Cambridge, MA: Harvard University Press, 1986, 1988 reprint. 1-85.

Cornell, Lauren. Taryn Simon's Visual Babel. The New Yorker. 2 August 2012. Web. 17 May 2015. <http://www.newyorker.com/culture/culture-desk/taryn-simons-visual-babel>

Cornu, Jean-Nicolas, Géraldine Cancel-Tassin, Valérie Ondet, Caroline Girardet, and Olivier Cussenot. Olfactory Detection of Prostate Cancer by Dogs Sniffing Urine: A Step Froward in Early Diagnosis. European Urology. 59.2 (February 2011):197-201. Web.

Cresswell, John. Qualitative Inquiry and Research Design: Choosing Among Five Approaches. $2^{\text {nd }}$ edition. Thousand Oaks and London: Sage Publications, 2007. Print.

Dam, H.J.W. The New Marvel in Photography. McCLure's Magazine. VI.5 (April 1896): 403415. Web.

Dear, Peter. A Mechanical Microcosm. Science Incarnate: Historical Embodiments of Natural Knowledge. Eds. Christopher Lawrence and Steven Shapin. Chicago and London: University of Chicago Press, 1998. 51-80. Print.

Debus, Allen George. The French Paracelsians: The Chemical Challenge to Medical and Scientific Tradition in Early Modern France. Cambridge, UK: Cambridge University Press, 2002. Web.

Donald, Ian, J. Macvicar, and T.G. Brown. Investigation of Abdominal Masses by Pulsed Ultrasound. The Lancet. 271.7032 (7 June 1958): 1188-1195. Web.

Doucet, Andrea and Natasha S. Mauthner. What can be known and how? Narrated subjects and the Listening Guide. Qualitative Research. 8 (2008): 399-409. Web. <DOI: 10.1177/1468794106093636>.

Drew, Trafton, Melissa L.-H. Võ, and Jeremy M. Wolfe. The Invisible Gorilla Strikes Again: Sustained Inattentional Blindness in Expert Observers. Psychological Science. XX.X (17 July 2013):1-6. Web. 13 September 2013. <DOI: 10.1177/0956797613479386>

Dyer, George S.M. and Mary E.L. Thorndike. Quidne Mortui Vivos Docent? The Evolving Purpose of Human Dissection in Medical Education. Academic Medicine. 75.10 (October 2000): 969-979. Web.

Eamon, William. The Professor of Secrets: Mystery, Medicine, and Alchemy in Renaissance Italy. Washington, DC: National Geographic Society, 2010. Print.

Eisenberg, Leon. Disease and Illness: Distinctions Between Professional and Popular Ideas of Sickness. Culture, Medicine and Psychiatry. 1.1 (1977): 9-23. Web. 
Emerson, Haven. Periodic medical examinations of apparently healthy persons. Journal of the American Medical Association. 80.19 (1923):1376-1381. Web.

Emerson, Joan P. Nothing Unusual is Happening. Human Nature and Collective Behaviours: Papers in Honor of Herbert Blume. Ed. Tamotsu Shibutani. Englewood Cliffs, NJ: Prentice-Hall, 1970. 208-222. Print.

Engel, George L. Are Medical Schools Neglecting Clinical Skills? Journal of the American Medical Association. 236.7(16 August 1976):861-863.

Epstein, Ronald M., Peter Franks, Kevin Fiscella, Cleveland G. Shields, Sean C. Meldrum, Richard L. Kravits, and Paul R. Duberstein. Measuring patient-centred communication in Patient-Physician consultations: Theoretical and practical issues. Social Science and Medicine. 61 (2005) 1516-1528. Web.

Environmental Protection Agency (EPA). Antimony Compounds. 18 October 2013. Web. $<$ http://www.epa.gov/ttnatw01/hlthef/antimony.html >

Eskinazi, Daniel. Factors That Shape Alternative Medicine. Journal of the American Medical Association. 280:18 (November 1998): 1621-1623. Web. <doi:10.1001/jama.280.18.1621>.

Every Man His Own Doctor or A Cure of the Human Body. 1835. Whitstable, UK: Pryor Publications, reprinted 1998. Print.

Fainzang, Sylvie. When doctors and patients lie to each other: Lying and power within the doctor-patient relationshiup. Lying and Illness: Power and Performacne. Eds. Els can Dongen and Sylvie Fainzang. Amsterdam: Het Spinhuis, 2005. 36-55. Print.

Fletcher, C.M. the Clinical Diagnosis of Pulmonary Emphysema-An Experimental Study. Proceedings of the Royal Society of Medicine. 45.9 (September 1952): 577-584. Web.

Foucault, Michel. Birth of the Clinic: An Archaeology of Medical Perception. Trans Tavistock Pub. Abingdon, Oxon, UK: Routledge, 1973. Print.

---. Discipline and Punish: The Birth of the Prison. Trans. Alan Sheridan. New York, NY: Random House, 1975. Print.

---. History of Madness. Trans. Jonathan Murphy and Jean Khalfa. London and New York: Routledge, 2006 (orig. 1961). Print.

---. The History of Sexuality: Volume I: An Introduction. Trans. Robert Hurley. New York, NY: Random House, 1980. Print. 
Frame, Paul. Shoe-Fitting Fluoroscope (ca. 1930-1940). Oak Ridge Associated Universities (ORAU) Health Physics Historical Instrumentation Museum Collection. 20 April 2010. Web. 〈http://www.orau.org/ptp/collection/shoefittingfluor/shoe.htm>.

Frank, Arthur. Letting Stories Breathe: A Socio-Narratology. Chicago, ILL: University of Chicago Press, 2010. Print.

---. Twin Nightmares of the Medical Simulacrum: Jean Baudrillard and David Cronenberg. Jean Baudrillard: The Disappearance of Art and Politics. Eds. William Stearns and William Chaloupka. New York, NY: St Martin’s Press, 1992, 82-97. Print.

---. The Wounded Storyteller: Body, Illness, and Ethics. $2^{\text {nd }}$ edition Chicago: University of Chicago Press, 2013 (1995). Print.

Fuller, R. Buckminster. The Phantom Captain. Nine Chains to the Moon. New York: Anchor Books, Doubleday and Co. 1971 (orig. 1938). 18-29.

Ganes, T. and T. Lundar. EEG and evoked potentials in comatose patients with severe brain damage. Electroencephalography and Clinical Neurophysicology. 69.1 (January 1988):613. Web.

Garrod, Archibald. The laboratory and the ward. Contributions to medical and biological research dedicated to Sir William Osler in honour of his seventieth birthday, July 12, 1919, by his pupils and co-workers. Ed. C.L. Dana. 1 (1919): 59-69. Web. 2 February 2014. <https://archive.org/stream/contributionstom01osleuoft\#page/64/mode/2up >

Gawande, Atul. Complications: A Surgeon's Notes on an Imperfect Science. New York: Picador, 2003. Web.

Gaylin, Willard. Harvesting the Dead. Harper's Magazine. (September, 1974): 23-28. Web. 18 December 2008. <http://faculty.uccb.ns.ca/philosophy/222/pdf\%20files/winter\%20stuff/9death.pdf $>$.

Geertz, Clifford. Thick Description: Toward an Interpretive Theory of Culture. The Interpretation of Cultures. New York: Basic Books, 1973. 3-30. Web.

Gladwell, Malcolm. ANNALS OF TECHNOLOGY: The Picture Problem: Mammography, air power, and the limits of looking. The New Yorker. 13 December 2004. Web. 16 April 2010. 〈http://www.gladwell.com/pdf/picture.pdf >.

Goodell, William. Clinical Notes on the Extirpation of the Ovaries for Insanity. The American Journal of Insanity. (January 1882): 294-302. Web. 20 January 2014. <http://books.google.ca/books?id=WkwXAQAAMAAJ\&lpg=PA294\&ots=X_XuomfPlp $\underline{\& \mathrm{dq}}=\% 22 \mathrm{Clinical} \% 20$ Notes $\% 20 \mathrm{on} \% 20$ the $\% 20$ Extirpation $\% 20 \mathrm{of} \% 20$ the $\% 20$ Ovaries $\% 2$ 
Ofor\%20Insanity.\%22William\%20Goodell\&pg=PA295\#v=onepage \&q=Goodell $\& \mathrm{f}=$ false $>$.

Goswami, Binita, Bhawna Singh, Ranjna Chawla, and Venkatesan Mallika. Evaluation of errors in a clinical laboratory: a one-year experience. Clinical Chemistry and Laboratory Medicine. Web. 48.1 (2010): 63-66.

Graham, Evarts A. Some functional tests and their significance. New England Journal of Medicine. 199 (5 July 1928): 1-7. Web.

Gray, Henry. Anatomy: Descriptive and Surgical. London, UK: John William Parker, 1858.

Gregory, S.R. and T.R. Cole. The changing role of dissection in medical education. Journal of the American Medical Association. 278.9 (6 March 6 2002):1180-1181. Web.

Groth-Petersen, E, A. Løvgreen, and J. Thillemann. On the reliability of the reading of photofluorograms and value of dual reading. Acta Tuberculosea Scandinavica. 26.1-2 (1952): 13-37. Web.

Gruver, Robert H. and Edward D. Freis. A Study of Diagnostic Errors. Annals of Internal Medicine. 47.1 (July 1957):108-120. Web.

Hampton, J.R., M.J.G. Harrison, J.R.A. Mitchell, J.S. Pritchard, and Carol Seymour. Relative contributions of History-taking, Physical Examination, and Laboratory Investigation to Diagnosis and Management of Medical Outpatients. British Medical Journal. 2:5969 (31 May 1975): 486-489. Web. 28 October 2010. <http://www.ncbi.nlm.nih.gov/pmc/articles/PMC1673456/>

Haraway, Donna.Modest_Witness@Second_Millennium: FemaleMan_Meets_OncoMouse. New York: Routledge, 1997. Print.

Hartzman, Marc. American Sideshow: An Encyclopedia of History's Most Wondrous and Curiously Strange Performers. New York: Penguin, 2005. Print.

Hayles, Katherine N. How We Became Posthuman: Virtual Bodies in Cybernetics, Literature, and Informatics. Chicago, IL: University of Chicago Press, 1999. Print.

Hawkins, Anne Hunsaker. Reconstructing Illness: Studies in Pathography. West Lafayette, IN: Perdue University Press, 1999. Print.

Herrick, James. Modern Diagnosis. Journal of the American Medical Association. $92: 7$ (16 February 1929): 518-522. Web. 
Horvath, György, Håkan Andersson, and Gunnar Paulsson. Characteristic odour in the blood reveals ovarian carcinoma. BioMed Central Cancer. 24 November 2010. 10:643. Web. <doi:10.1186/1471-2407-10-643>.

House Rules, Houseisms: Rules, Proverbs, Philosophy, Quotes. House: Guide to the TV Show. n.d. Web. 3 May 2015. http://www.housemd-guide.com/characters/houserules.php

Human Genome Project. Human Genome Project Information Archive. n.d. Web. 20 January 2014. <http://web.ornl.gov/sci/techresources/Human_Genome/index.shtml>.

Ihde, Don. Bodies in Technology. Minneapolis, MN: University of Minnesota Press, 2001. Print.

Illich, Ian. Medical Nemesis: The Expropriation of Health. London: Marion Boyars, 1975. Print.

InSitu Foundation. 2013. Web. 23 September 2013. <http://www.dogsdetectcancer.org/>.

International Eugenics Congresses (IEC). Curators of the University of Missouri Last. 16 March 2012. Web. 25 January 2014.

<http://library.missouri.edu/exhibits/eugenics/congresses.htm>.

Jenkins, Catherine. Life Extension, Immortality and the Patient Voice. The Power of Death: Perceptions of Death in the Western World. Eds. Maria-José Blanco and Ricarda Vidal. Oxford, UK: Berghahn Books, 2014.

---. The Message in Medical Imaging Media: An Analysis of GE Healthcare's Vscan ${ }^{\mathrm{TM}}$. Finding McLuhan: The Mind/The Man/The Message. Eds. Jaqueline McLeod Rogers, Tracy Whalen, and Catherine G. Taylor. Regina, SK: University of Regina Press, 2015. Print.

Jenkins, Isobel. Unpublished memoir, 1994.

Jenkins, John D. Crookes and Geissler Tubes. Spark Museum: Early Radio and Scientific Apparatus. n.d. Web. 3 March 2014. 〈http://www.sparkmuseum.com/GLASS.HTM>.

Jewson, Nicholas D. The disappearance of the sick-man from medical cosmology 1770-1870. Sociology 10 (1976): 225-244. Web.

Johnson, Peter C. Guy de Chaulliac and the Grand Surgery. Surgery, Gynecology \& Obstetrics. 169: 2(August 1989): 172-176. Web.

Jørgensen, Marianne and Louise Phillips. Discourse Analysis as Theory and Method. London, Thousand Oaks, New Delhi: Sage Publications2002. Print.

Journal of the American Medical Association. About JAMA. n.d. Web. $<$ http://jama.jamanetwork.com/public/About.aspx> 
Joyce, Kelly. Appealing Images: Magnetic Resonance Imaging and the Production of Authoritative Knowledge. Social Studies of Science. 35.3(June 2005): 437-462. Web. <DOI: $10.1177 / 0306312705050180$ >.

Kalager, Metter, Hans-Olov Adami, Michael Bretthauer, and Rulla Tamimi. Overdiagnosis of Invasive Breast Cancer Due to Mammography Screening: Results From the Norwegian Screening Program. Annals of Internal Medicine. 156.7 (3 April 2013): 491. Web. <http://ezproxy.lib.ryerson.ca/login?url=http://search.proquest.com.ezproxy.lib.ryerson.c a/docview/1004095830?accountid=13631>.

Kesey, Ken. One Flew Over the Cuckoo's Nest. New York: Signet, 1962.

Kevles, Bettyann Holtzmann. Naked to the Bone: Medical Imaging in the Twentieth Century. New Brunswick, NJ: Rutgers University Press, 1997. Print.

Kidney Foundation Organ Donation Overview. Kidney Foundation of Canada. n.d. Web. 18 April 2009. < http://www.kidney.ca/page.aspx?pid=375 >.

King, Thomas. The Truth About Stories: A Native Narrative. Toronto: House of Anansi Press, 2003. Print.

Kinnersley, Paul, Nigel Stott, Tim J. Peters, and Ian Harvey. The patient-centredness of consultations and outcome in primary care. British Journal of General Practice. 49 (September 1999): 711-716. Web.

Kleinman, Arthur. What is Specific to Western Medicine? Companion Encyclopedia of the History of Medicine. Eds. W.F. Bynum and Roy Porter. New York, NY: Routledge, 1993. I, 15-23. Print.

Knoblauch, Hubert. Focused Ethnography. Forum: Qualitative Social Research. 6:3 (September 2005) Art. 44. Web. 5 October 2009. <http://www.qualitativeresearch.net/index.php/fqs/article/view/20/43>.

Kolata, Gina. Sports Medicine Said to Overuse a Popular Scan. The New York Times. 28 October 2011. Web. 29 October 29 2011. <http://www.nytimes.com/2011/10/29/health/mrisoften-overused-often-mislead-doctorswarn.html?pagewanted=1\&_r=1\&partner=rss\&emc=rss\&src=ig >

Korley, Frederick Kofi, Julius Cuong Pham, and Thomas Dean Kirsch. Use of advanced radiology during visits to US emergency departments for injury-related conditions, 19982007. Journal of the American Medical Association. 304.13 (6 October 2010) :14651471. Web.

Korran, Lorrin M. The reliability of clinical methods, data and judgements. New England Journal of Medicine. 293 (October 1975) : 695-701. Web. 
Koutalos, Yiannis. The Dolice Body of the Scientist. The Mangle of Practice: Science, Society, and Becoming. Eds. Andrew Pickering and Keith Guzik. Durham, NC: Duke University Press, 2009. 202-218. Print.

Krupinski, Elizabeth A. The Importance of Perception Research in Medical Imaging. Radiation Medicine. 18.6 (12 June 2000): 329-334. Web.

Kuriyama, Shigehisa. Interpreting the History of Bloodletting. Journal of the History of Medicine and Allied Sciences. 50(1995): 11-46. Web.

Laine, Christine and Frank Davidoff. Patient-Centered Medicine: A Professional Evolution. Journal of the American Medical Association. 275.2 (10 January 1996): 152-156. Web. 28 July 2011. <http://ovidsp.tx.ovid.com.ezproxy.lib.ryerson.ca/sp3.4.1b/ovidweb.cgi? \&S=KHDNFPCEMODDNMJINCBLFALBLLMJAA00\&Link+Set= jb.search.26\%7c1\%7cs1_10\#14 >.

Leder, Drew. A Tale of Two Bodies: The Cartesian corpse and the Lived Body. The Body in Medical Thought and Practice. Ed. Drew Leder. Boston, IL: Kluwer Academic Publishers, 1992. 17-35. Print.

Lee, Yin-Yang and Julia Lin. Do patient autonomy preferences matter? Linking patient-centered care to patient-physician relationships and health outcomes. Social Science and Medicine. 71.10 (2010): 1811-1818. Web.

Lehoux, Pascale. The Problem of Health Technology: Policy Implications for Modern Health Care Systems. New York: Routledge, 2006. Print.

Lewisham Healthcare NHS Trust. The Choir: Sing While You Work. 1:1 BBC. 20 Sept. 2012. Television.

Li, Han Z., Corinne Koehn, Naghmeh G. Desroshes, Young-Ok Yum, and George Deagle. Asymmetrical Talk between Physicians and Patients: A quantitative Discourse Analysis. Canadind Journal of Communcation. 32 (2007): 417-433. Web.

Liang, Wenchi, Deborah Kasman, Judy H. Wang, Elaine H. Yuan, and Jeanne S. Mandelblatt. Communication between older women and physicians: Preliminary implications for satisfaction and intention to have mammorgaphy. Patient Education and Counceling. 64 (2006): 387-392. Web. < doi:10.1016/j.pec.2006.04.004>

Liptak, Adam. Justices, 9-0, Bar Patenting Human Genes. The New York Times. 13 June 2013. Web. 20 January 2014. 〈http://www.nytimes.com/2013/06/14/us/supreme-court-ruleshuman-genes-may-not-be-patented.html?hp\&_r=1\&>. 
Little, P., Everitt, H., Williamson, G., Warner, G., Moore, M., Gould, C., Ferrier, K., Payne, S.

Preferences of patients for patient centred approach to consultation in primary care: observational study. British Medical Journal. 322:7284 (2001) 468. Web.

Lock, Margaret. Licorice in Leviathan: The Medicalization of Care for the Japanese Elderly. Culture, Medicine and Psychiatry. 8:2 (June 1984): 121-139. Web. 6 July 2012. <http://journals2.scholarsportal.info.ezproxy.lib.ryerson.ca/tmp/17851556763927378056. pdf>.

--- and Vihn-Kim Nguyyen. Biomedical Technologies in Practice. An Anthology of Biomedicine. Malden, MA and Oxford, UK: John Wiley \& Sons, 2010. 17-31. Print.

Locsin, Rozzano C. Technological Competency as Caring in Nursing: A Model for Practice. Indianapolis: Sigma Theta Tau International, 2005. Print.

Loop Demand Gen, LLC. n.d. Web. 23 December 2014. < http://loopdemandgen.com/blog/coldcalling-outperforms-e-mail-and-direct-mail/>>

Magliocco, Sabina. Spells, Saints, and Streghe: Witchcraft, Folk Magic, and Healing in Italy. The Pomegranate. 13 (2000): 2-22. Web. 8 September 2011. <http://www.italiansrus.com/articles/subs/folkmagic.htm >.

Matsuda, W, A. Matsumura, Y. Komatsu, K. Yanaka, and T. Nose. Awakenings from persistent vegetative state: report of three cases with parkinsonism and brain stem lesions on MRI. Journal Neurology Neurosurgery and Psychiatry. 74 (2003): 1571-1573. Web. <doi:10.1136/jnnp.74.11.1571>

Mayo Clinic. Aphasia, Definition. n.d. Web. 2 March 2014. <http://www.mayoclinic.org/diseases-conditions/aphasia/basics/definition/con$\underline{20027061>}$.

Mayo Clinic. Bone Density, Definition. n.d. Web. 20 June 2014. < http://www.mayoclinic.org/tests-procedures/bone-density-test/basics/definition/prc20020254.>

Mayo Clinic. Bone Scan, Definition. n.d. Web. 20 June 2014. < http://www.mayoclinic.org/testsprocedures/bone-scan/basics/why-its-done/prc-20020302>.

McCulloch, Michael, Tadeusz Jezierski, Michael Broffman, Alan Hubbard, Kirk Turner, and Teresa Janecki. Diagnostic accuracy of canine scent detection in early-and-late-stage lung and breast cancers. Integrated Cancer Therapy. 5.1 (2006):30-39. Web.

McDaniel, Brock and Farrokh Dehdashti. Teaching File Case number: pt 096. Mallinckrodt Institute of Radiology, Washington University. Web. 31 Dec 2012. <http://gamma.wustl.edu/pt096te163.html >. 
McKenna, Maryn. Virtues of the Virtual Autopsy: Medical imaging offers new ways to examine the deceased. Scientific American. 307.5 (16 October 2012). Web. <http://www.scientificamerican.com/article/virtues-of-the-virtual-autopsy/ >.

Mead, Nicola, and Peter Bower. Patient-centredness: a conceptual framework and review of the empirical literature. Social Science and Medicine. 51:7(2000): 1087-1110. Web.

Means, Hugh J. The uses and limitations of the X-ray. Ohio State Medical Journal. 19 (1923): 792-5. Web.

Mehrabian, Albert. Silent messages: Implicit communication of emotions and attitudes. Belmont, CA: Wadsworth, 1981. Web. <http://www.kaaj.com/psych/smorder.html>.

Michener Institute. Web. 20 February 2015. < http://michener.ca/>.

Mishler, Elliot G. Ed. Social contexts of health, illness, and patient care. Cambridge, UK and New York, NY: Cambridge University Press, 1981. Print.

Mol, Annemarie and John Law. Embodied action, enacted bodies: The example of hypoglycaemia. Biomedicine as Culture: Instrumental Practices, Technoscientific Knowledge, and New Modes of Life. Eds. Regula Valérie Burri and Joseph Dumit. New York, London: Routledge, 2007. 87-107. Web.

More or Less Medicine. The Agenda with Steve Paikin. TVOntario. TVO, Toronto. 5 Feb. 2013. Television. <http://ww3.tvo.org/video/187922/more-or-less-medicine>.

Morris, David. Illness and Culture in the Postmodern Age. University of California Press, 1998. Print.

Morton, William J. and Edwin W. Hammer. The X Ray or Photography of the Invisible and its Value in surgery. New York: American Technical Book Co., 1896. Web. <http://archive.org/stream/xrayorphotograp01hammgoog\#page/n6/mode/2up >.

Moser, Emily and Michael McCulloch. Canine Scent Detection of Human Cancers: a Review of Methods and Accuracy. Journal of Veterinary Behaviour . 5.3 (2010):145-152.Web.

Mullins, Andrew. Case 12100. MedPx TM Medical Image Database, Atlas, and Teaching File, Department of Radiology and Rodiological Sciences, Uniformed Services University (Bethesda, MD). Web. 31 Dec 2012.

<http://rad.usuhs.mil/medpix/tf_case.html?mode=case_viewer\&case_prog=cow_image.h $\underline{\mathrm{tml} \& \mathrm{th}=-}$

$1 \&$ quiz=yes\&pt $\mathrm{id}=12100 \& \mathrm{imid}=0 \& \mathrm{imageid}=43802 \&$ week $=\& \max =\&$ skiprows $=\& \operatorname{maxc}$ $\underline{\text { ases }=\& \text { skipcases }=\& \text { this_week }=\& \text { conf }=\# \text { clinical }>}$ 
Naci, L., M.Monti, D. Cruse, A. Kubler, B. Sorger, R. Goebel, B. Kotchoubey, and A.M. Owen. Brain computer interfaces for communication with non-responsive patents. Annals of Neorology. 72.3 (2012): 312-323. Web.

National Cancer Institute (NCI). Risks of Breast Cancer Screening. 3 December 2013. Web. 3 March 2014. <http://www.cancer.gov/cancertopics/pdq/screening/breast/Patient/page4>.

New York Times. Her Latest Photograph.29 May 1898. Web.

<http://query.nytimes.com/mem/archivefree/pdf?res=F10D14FD3A5D11738DDDA00A94DD405B8885F0D3

Ofri, Danielle. The Physicial Exam as Refuge. New York Times. 10 July 2014. Web. <http://well.blogs.nytimes.com/2014/07/10/the-physical-exam-asrefuge/?_php=true \&_type $=$ blogs \& $r=1>$.

Operating Theatre. Old Operating Theatre Museum and Herb Garret. 30 November 2012. Web. <http://www.thegarret.org.uk/oot.htm>.

Ongaro, Giuseppe. Medicine. The University of Padua: Eight Centuries of History. Ed. Piero Del Negro. Trans. Hilary Siddons and Helen Deborah Walberg. Padova, Italy: Signum Padova, 2001-2003. 153-193. Print.

Ontario Ministry of Finance. Ontario Health insurance Program-VOTE 1405. 2010. Web. <http://www.fin.gov.on.ca/en/budget/estimates/201415/volume1/MOHLTC_2181.html>.

Ontario Ministry of Health and Long-Term Care. Interprovincial Out-Patient Rates, Effective April 1, 2014. Web.

<http://www.health.gov.on.ca/en/pro/programs/ohip/bulletins/4000/bul4627_1.pdf July 8 2014-07-08 >.

Ontario Ministry of Health and Long-Term Care. Ministry History. 25 June 2012.

Web. 〈http://www.health.gov.on.ca/en/common/ministry/history.aspx $>$.

Open Arms Patient Advocacy Society. n.d. Web. 18 July 2014. <http://www.openarmsadvocacy.com/index.html $>$.

Orenstein, Beth. Ultrasound History. Radiology Today. 9.24 (1 December 2008): 28. Web. 5 January 2013. 〈http://www.radiologytoday.net/archive/rt_120108p28.shtml>.

Osherson, Samuel and Lorna AmaraSingham. The Machine Metaphor in Medicine. Social Contexts of Health, Illness, and Patient Care. Ed. Eliot G. Mishler. Cambridge, UK and New York, NY: Cambridge University Press, 1981. 218-248. Print. 
Owen, Adrian M, Martin R. Colman, Melanie Boly, Mathew H. David, Steven Laureys, and John D. Pickard. Using Fuctional Magnetic Resonance Imaging to Detect Covert Awareness in the Vegetative State. Archives of Neorology. 64.8 (August 2007):10981102. Web. <doi:10.1001/archneur.64.8.1098. >

Owen Lab.n.d. Web. 〈http://owenlab.org/?page_id=16>.

Parisi, J.E., R.C. Kim, G.H. Collins, and M.F. Hilfinger. Brain death with prolonged somatic survival. New England Journnal of Medicine. 306.1 (7 January 1982):14-16. Web

Patients Canada. 2013-2015. Web. 18 July 2014. <http://www.patientscanada.ca/>.

Pauwels, Luc. An integrated model for conceptualising visual competence in scientific research and communication. Visual Studies. 23.2 (September 2008): 147-161. Web. 8 February 2012. 〈http://dx.doi.org/10.1080/14725860802276305>.

Pedretti, Carlo and Catherine Frost. Anatomy: The Body in Art and in Science. Leonardo: Art and Science. Milan, Italy: Giunti Editore, 2000. 110-125. Print.

Peterkin, Allan. Practical strategies for practising narrative-based medicine. Canadian Family Physician. 58 (January 2012): 63-64. Web.

Petrini, John L. Fools rush in... Gastrointestinal Endoscopy. 63.3 (2006): 383-384. Web. 21 July 2014. <http://jama.jamanetwork.com/article.aspx?articleid=370166 >.

Pickering, Thomas G., Gary D. James, Charlene Boddie, Gregory A. Harshfield, Seymour Blank, and John H. Laragh. How Common is White Coat Hypertension? Journal of the American Medical Association. 259.2 (1988): 225-228. Web. doi:10.1001/jama.1988.03720020027031>.

Pine Street Foundation. December 2012. Web. 19 August 2013. $<$ http://pinestreetfoundation.org/category/canine-scent-detection/>.

Plebani, Mario. Errors in clinical laboratories or errors in laboratory medicine? Clinical Chemistry and Laboratory Medicine. 44.6 (2006):750-759. Web.

Plymouth University Peninsula, School of Medicine. n.d. Web. 22 June 2015. <https://www.plymouth.ac.uk/schools/peninsula-school-of-medicine $>$.

Poole, Jennifer M., Margrit Shildrick, Patricia McKeever, Susan Abbey, and Heather Ross. "You Might Not Feel Like Yourself:" On Heart Transplants, Identity and Ethics. Critical Interventions in the Ethics of Healthcare: Challenging the Principles of Autonomy in Bioethics. Eds. Stuart J. Murray and Dave Holmes. Farnham, Surrey, UK: Ashgate, 2009. 33-44. Print. 
Porter, Roy. Blood and Guts: A Short History of Medicine. London, UK: Penguin Books, 2002. Print.

---. Ed. The Cambridge History of Medicine. New York: Cambridge University Press, 2006. Print.

---. The Greatest Benefit to Mankind: A Medical History of Humanity. New York, NY and London, UK: W.W. Norton \& Co, 1997. Print.

--- Ed. Patients and Practitioners: Lay Perceptions of Medicine in Pre-Industrial Society._New York, NY: Cambridge University Press, 1985. Web.

Potchen, E. James. Measuring Observer Performance in Chest Radiology: Some Experiences. Journal of the American College of Radiology.3 (2006):423-432. Web. 9 February 2014. < DOI 10.1016/j.jacr.2006.02.020. >

Prado, Ariadne Juana Fernandes do, Ediane Arimatéa Silva, Vinícius Anjos de Almeida, and Renério Fráguas Júnior. Medical environment: bad news' impact on patients and doctors - towards an effective model of communication. Review of Medicine (São Paulo). 92.1 ( January-March 2013):13-24. Web.

Prasad, Amit. Making Images/Making Bodies: Visibilizing and Disciplining through Magnetic Resonance Imaging (MRI). Science, Technology, and Human Values. 30.2 (Spring 2005): Web. <291-316. DOI: 10.1177/0162243904271758>.

Price, David L., Janet P. De Wilde, Annie M. Papadaki, Jane S. Curran and Richard I. Kitney. Investigation of acoustic noise on $15 \mathrm{MRI}$ scanners from $0.2 \mathrm{~T}$ to $3 \mathrm{~T}$. Journal of Magnetic Resonance Imaging. 13.2 (February 2001): 288-93. Web.

Pruitt Jr, Basil A. Combat Casualty Care and Surgical Progress. Annals of Surgery. 243:6 (June 2006): 715-729. Web. <doi: 10.1097/01.sla.0000220038.66466.b5>.

Radstake, Maud . Visions of illness: An endography of real-time medical imaging. Delft, Netherlands: Eburon, 2007. Print.

Rando, Therese. Living and Learning with the Reality of a Loved One's dying: Traumatic Stress and Cognitive Processing of Anticipatory Grief. Living with Grief: When Illness is Prolonged. Ed. Kenneth Doka. Washington, DC: Hospice Foundation of America through Taylor and Francis Publishers, 1997. 33-50. Web.

Reilly, J.Patrick. Peripheral nerve stimulation by induced electric currents: exposure to timevarying magnetic fields. Medical and Biological Engineering and Computing. 27.2 (March 1989): 101-10. Web. 〈doi:10.1007/BF02446217. PMID 2689806〉. 
Reiser, Stanley Joel. Medicine and the Reign of Technology. Cambridge UK and New York, NY: Cambridge University Press, 1978. Print.

---. Technological Medicine: The Changing World of Doctors and Patients. New York: Cambridge University Press, 2009. Print.

---. Technology and the eclipse of individual in medicine. The Pharos. (Winter 1982): 10-15. Web.

Robertson, Gerald. Eugenics. The Canadian Encyclopedia. Toronto: Historical Canada, 2006. Web. 25 January 2014. 〈http://www.thecanadianencyclopedia.ca/en/article/eugenics/>

Rogers, Carl. Client-Centered Therapy. Boston: Houghton Mifflin, 1951. Print.

Roosen, John, Eric Frans, Alexander Wilmer, Daniel C. Knockaert, and Herman Bobbaers. Comparison of Premortem Clinical Diagnoses in Critically Ill Patients and Subsequent Autopsy Findings. Mayo Clinic Proceedings. 75 (June 2000): 562-567. Web.

Rose, Nikolas S. The Politics of Life Itself: Biomedicine, Power, and Subjectivity in the Twentyfirst Century. Princeton: Princeton University Press, 2007. Print.

Rosenberg, Leah. Does Direct-to-Consumer Marketing of Medical Technologies Undermine the Physician-Patient Relationship? The American Journal of Bioethics. 9.4 (April 2009): 22-23. Web.

Roter, Debra L., Miora Stewart, Samuel M. Putnam, Mack Lipkin, William Stiles, and T. S. Inui. Communication patterns of primary care physicians. Journal of the American Medical Association. 277 (1997): 350-356. Web.

St. Joseph MRI. n.d. Web. 〈http://www.stjosephmri.com/index.php?page=price_list.html〉.

Sandelowski, Margarete. Devices and Desires: Gender, Technology, and American Nursing Chapel Hill and London: University of North Carolina Press, 2000. Print.

Selden, Steven. Transforming Better Babies into Fitter Families: Archival Resources and the History of the American Eugenics Movement, 1908-1930. Proceedings of the American Philosophical Society. 149. 2 (June 2005): 199-225. Web. 25 January 2014. 〈http://www.jstor.org/stable/4598925>.

Service Canada. Canada Pension Plan.7 September 2014. Web. 17 January 2012. <http://www.servicecanada.gc.ca/eng/services/pensions/cpp/index.shtml>

Shaw, George Bernard. The Doctor's Dilemma. London, UK: Penguin, 1987 (original play1906).

Shewmon, D. Alan. Brain Death: Can It Be Resuscitated? Hasting Center Report. 2992 (MarchApril 2009): 18-24. Web. <DOI: 10.1353/hcr.0.0122>. 
---. The Brain and Somatic Integration: Insights Into the Standard Biological Rationale for Equating "Brain Death" With Death. The Journal of Medicine and Philosophy. 26.5 2001): 457-478. Web. <doi: 10.1076/jmep.26.5.457.3000>.

Shogan, Paul. Case1 1498 MedPx TM Medical Image Database, Atlas, and Teaching File, Department of Radiology and Radiological Sciences, Uniformed Services University (Bethesda, MD). Web. 4 January 2013.

$<$ http://rad.usuhs.edu/medpix/cow_image.html?quiz $=\& p t \_i d=11498 \&$ imageid=39557\&w idgets $=-1 \&$ start $=-1 \&$ mode $=\#$ top $>$ and $<$ http://rad.usuhs.edu/medpix/tf_case.html?mode=case_viewer\&case_prog=cow_image.h tml\&th=$1 \& q u i z=\& p t \_i d=11498 \& i m i d=39561 \&$ imageid $=39561 \&$ week $=\& \max =\&$ skiprows $=\& m a$

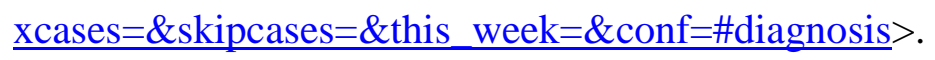

Simons, Daniel and Christopher Chabris. The invisible gorilla. 1999. Web. 30 May 2014. 〈http://www.theinvisiblegorilla.com/videos.html $>$.

Simpson, Michael, Robert Buckman, Moira Stewart, Peter Maguire, Mack Lipkin, Dennis Novak, and James Till. Doctor-patient communication: the Toronto consensus statement. British Medical Journal. 303.6814 (30 November 1991):1385-1387. Web. 28 October 2010. 〈http://www.ncbi.nlm.nih.gov/pmc/articles/PMC1671610/>.

Smith, F.C. Cough during auscultation in the diagnosis of pulmonary tuberculosis: A plea for its routine employment to elicit fine râles:--technic and convenient signal. Journal of the American Medical Association. LVI.4 (28 January 1911.):245. Web. <doi:10.1001/jama.1911.02560040013004. >.

Sonoda, Hideto, Shunji Kohnoe, Tetsuro Yamazato, Yuji Satoh, Gouki Morizono, Kentaro Shikata, Makoto Morita, Akihiro Watanabe, Masaru Morita, Yoshihiro Kakeji, Fumio Inoue, and Yoshihiko Maehara. Colorectal cancer screening with odour material by canine scent detection. Gut: An international peer-reviewed journal for health professionals and researchers in gastroenterology and hepatology. 31 January 2011. Web. <doi:10.1136/gut.2010.218305>.

Sonoma State University, Health Navigator Program. n.d. Web. 18 July 2014. $\langle$ http://www.sonoma.edu/exed/patient-navigator/ >.

Statistics Canada. Age-standardized mortality rates by selected causes, by sex. Web. 25 July 2011. 〈http://www40.statcan.gc.ca/101/cst01/health30a-eng.htm>.

Stewart, Alice. Malignant Disease in Childhood and Diagnostic Irradiation in Utero. The Lancet. 268.6940 (1 September 1956): 447. Web. <doi:10.1016/S0140-6736(56)91923-7>. 
Stradling, Peter and R.N. Johnston. Reducing Observer Error in a 70-mm. Chest-Radiography Service for General Practitioners. The Lancet. 265.6877 (18 June 1955): 1247-1250. Web.

Street Jr, Richard L. and Howard S. Gordon. The clinical context and patient participation in post-diagnostic conscultations. Patient Education and Councelling. 64 (2006): 217-224. Web.

Stubbs, Barbara. Interviewed in Med-Ed News. Toronto: University of Toronto's Faculty of Medicine. (February 1986). Print.

Taylor, Paul. Doctors need to take customer service seriously for patient-centred care to work. Healthy Debate. 2014. Web. <http://healthydebate.ca/personal-health-navigator/patientcentred-care>.

University College of London. The Wolfson Institute for Biomedical Research. 1999-2015. Web. 8 January 2014. <http://www.ucl.ac.uk/>.

---. Cruciform Building. 1999-2015. Web. 8 January 2014. <http://www.ucl.ac.uk/wibr/building/cruciform/index.htm>.

University of Toledo. Graduate Certificate in Patient Advocacy. n.d. Web. 18 July 2014. $<$ http://www.utoledo.edu/online-degreeprograms/programs/certificates/cert_advocacy.html >.

Van Praagh, Richard and Stella Van Praagh. Artistotle's 'Triventricular' Heart and the Relevant Early History of the Cardiovascular System. Chest, 84:4 (October 1983): 462-468. Web. <http://journal.publications.chestnet.org/data/Journals/CHEST/21379/462.pdf >.

van Dijck, José. The Transparent Body: A Cultural Analysis of Medical Imaging. Seattle, WA: University of Washington Press, 2005. Print.

Vaughn, Douglas, Ed. A Vital Legacy: Biological and Environmental Research in the Atomic Age. U.S. Department of Energy, The Office of Biological and Environmental Research, September 1997. Web. 20 January 2013. <http://www.escholarship.org/uc/item/9vv2404w >.

Verghese, Abraham. Empathy: Good for Doctors and Bad for Judges? The Atlantic. 27 May 2009. Web. 4 April 2015.

<http://www.theatlantic.com/technology/archive/2009/05/empathy-good-for-doctors-andbad-for-judges/18375/>.

---. Under-told stories. 29 July 2010. Web. 4 April 2015. <http://www.undertoldstories.org/stories/doctor-stresses-intuition-touch-not-technology >. 
---. Religion and Ethics Newsweekly. Public Broadcasting Service (PBS). 24 November 2010. Web. 4 April 2015. <http://www.pbs.org/wnet/religionandethics/2010/11/24/november26-2010-dr-abraham-verghese/7570/ >.

Verheijde, Joseph L, Mohamed Y. Rady, and Joan L. McGregor. Brain death, states of imparied consciousness, and physician-assisted death for end-of-life organ donation and tranplatation. Medicine, Health Care and Philosophy. 1.4 (November 2009): 409-421. Web.

Vesalius, Andreas. The Bloodletting Letter of 1539: An Annotated Translation and Study of the Evolution of Vesalius's Scientific Development. Eds. John B. de C.M. Saunders and Charles Donald O’Malley. New York, NY: Henry Schuman, 1969. Print.

---. De humani corporis fabrica libri septem.Joannes Oporinus, Basle: 1543. Print. (access courtesy of the Thomas Fisher Library at the University of Toronto)

---. On the Fabric of the Human Body: Book I The Bones and Cartilages. Trans. William Frank Richardson and John Burd Carman. San Francisco, CA: Norman Publishing, 1998 (orig. 1543). Print.

Visible Human Project. National Institutes of Health. 11 September 2003. Web. 8 January 2011. $<$ https://www.nlm.nih.gov/research/visible/visible_gallery.html>

Waldby, Catherine. The Visible Human Project: Informatic Bodies and Posthuman Medicine. New York, NY: Routledge, 2000. Print.

Wall, Shelley. The View from Inside: Gendered Embodiment and the Medical Representation of Sex. Critical Interventions in the Ethics of Healthcare: Challenging the Principles of Autonomy in Bioethics. Eds. Stuart J. Murray and Dave Holmes. Farnham, Surrey, UK: Ashgate, 2009. 133-146. Print.

Wallace, Daniel. Big Fish: A Novel of Mythic Proportions. New York, NY: Penguin Books, 1998. Print.

Wellcome Image of the Month: The left hand of Anna Roentgen. Wellcome Trust. 13 August 2010. Web. 12 September 2013. < http://blog.wellcome.ac.uk/2010/08/13/wellcomeimage-of-the-month-the-left-hand-of-anna-roentgen/ > .

Williams, Simon J. Modern Medicine and the 'Uncertain Body': From Corporeality to Hyperreality? Social Science and Medicine. 45.7 (197): 1041-1049. Web.

Willis, Carolyn M., Susannah M. Church, Claire M. Guest, W. Andrew Cook, Noel McCarthy, Anthea J. Bransbury, Martin R. T. Church, and John C. T. Church. Olfactory detection of human bladder cancer by dogs: proof of principle study. British Medical Journal. 32923 September 2004): 712. Web. <doi: http://dx.doi.org/10.1136/bmj.329.7468.712>. 
Wilson, James. What is Adrenal Fatigue? Adrenal Fatigue. 2014. Web. 2 March 2014. <https://www.adrenalfatigue.org/what-is-adrenal-fatigue>

---. Adrenal Fatigue: The 21st Century Stress Syndrome. Petaluma, CA: Smart Publications 2001. Print.

Wootton, David. Bad Medicine: Doctors Doing Harm since Hippocrates. Oxford, UK and New York, NY: Oxford University Press, 2006. Print.

World Health Organization. Definition of an older or elderly person. (n.d.) Web. 17 January 2012. < http://www.who.int/healthinfo/survey/ageingdefnolder/en/>

World Health Organization. Ageing and Life Course: Facts about ageing. 30 September 2014. Web. 17 March 2015. <http://www.who.int/ageing/about/facts/en/ >.

Worsley, Peter. Non-Western Medical Systems. Annual Review of Anthropology. 11 (1982): 315-348. Web. 